\title{
Supporting Secondary Mathematics Teacher Candidates in Enacting Responsive Teaching across Contexts.
}

\author{
Stephanie Jones
}

Follow this and additional works at: https://researchrepository.wvu.edu/etd

\section{Recommended Citation}

Jones, Stephanie, "Supporting Secondary Mathematics Teacher Candidates in Enacting Responsive Teaching across Contexts." (2018). Graduate Theses, Dissertations, and Problem Reports. 8207. https://researchrepository.wvu.edu/etd/8207

This Dissertation is protected by copyright and/or related rights. It has been brought to you by the The Research Repository @ WVU with permission from the rights-holder(s). You are free to use this Dissertation in any way that is permitted by the copyright and related rights legislation that applies to your use. For other uses you must obtain permission from the rights-holder(s) directly, unless additional rights are indicated by a Creative Commons license in the record and/ or on the work itself. This Dissertation has been accepted for inclusion in WVU Graduate Theses, Dissertations, and Problem Reports collection by an authorized administrator of The Research Repository @ WVU.

For more information, please contact researchrepository@mail.wvu.edu. 


\title{
Supporting Secondary Mathematics Teacher Candidates in Enacting Responsive Teaching Across Contexts
}

\author{
Stephanie Jones
}

\author{
Dissertation submitted \\ to the College of Education and Human Services \\ at West Virginia University \\ in partial fulfillment of the requirements for the degree of \\ Doctor of Education in \\ Curriculum \& Instruction \\ Johnna Bolyard, Ph.D., Chair \\ Matthew Campbell, Ph.D., Chair \\ Malayna Bernstein, Ph.D. \\ Melissa Luna, Ph.D. \\ Sarah Selmer, Ed.D. \\ Department of Curriculum and Instruction/Literacy Studies
}

\author{
Morgantown, West Virginia \\ 2018
}

Keywords: Secondary Mathematics, Teacher Candidates, Teacher Education,

Responsive Teaching, Pedagogies of Practice

Copyright 2018 Stephanie Jones 


\section{Abstract \\ Supporting Secondary Mathematics Teacher Candidates \\ in Enacting Responsive Teaching Across Contexts}

Stephanie Jones

Although the work of teaching is often considered universally straightforward, professional classroom teaching requires more than a teacher's ability to complete a task; making students' ideas central to classroom discussions is crucial to student learning. The ability to engage students in classroom discussions focused on student ideas is not innate; it must be developed. Thus, it is important for teacher candidates (TCs) to learn to focus class discussions on student ideas in the university setting, before becoming classroom teachers.

This dissertation investigates the ways in which secondary mathematics TCs developed skill in responsive teaching - teaching in which teachers adapt their instruction based on attention to students' ideas - in a methods course. The TCs in this study participated in cycles of investigation and enactment, focused around coached rehearsals of instructional activities (IAs) chosen to allow a focus on responsive teaching practices - core practices of teaching that have responsive teaching as their focus. In addition to investigating TCs' responsive teaching in methods, this study also examined how coaching in methods affected TCs' responsive teaching. Each TC also enacted one IA in a school setting, which allowed for a consideration of the extent to which TCs taught responsively in school settings, as compared to the university setting.

Previous methods course designs, both from the literature and a pilot study, informed both the design of the methods course as well as the research design of this study. Video recordings of TCs' enactments in the methods course and in the school classroom served as the primary data source, while TCs' video annotations and interview responses, along with my own reflective journal provided supporting data. Video analysis was conducted using Studiocode software to identify and organize salient moments in TCs' responsive teaching.

Findings revealed that TCs' development of responsive teaching skills took a variety of forms, such as better anticipating student reasoning and attempting to enact responsive teaching moves that were new to them. Findings also revealed that teaching moves positioned as responsive in the literature are not always enacted in responsive ways, highlighting the importance of TCs enacting these moves purposefully and judiciously. Coaching aided TCs in their development of responsive teaching skills by introducing students to new teaching moves (e.g., Ask Different $S$ to Revoice) and helping them to enact familiar moves in more responsive ways (e.g., $T$ Records). Finally, findings relative to the school setting indicated that TCs enacted many of the same teaching moves in methods and in schools, but in different ways, some more responsively than others. Implications of these findings for TE practice include engaging TCs in more focused discussions about planning for IAs, as well as maintaining a focus on responsive teaching in coaching interjections, in part by raising TCs' awareness of what might be gained by using teaching moves in particular ways. 


\section{Acknowledgements}

I would first like to thank Dr. Johnna Bolyard and Dr. Matthew Campbell, my dissertation co-chairs. Johnna has provided guidance from the very beginning of this process. She found the perfect balance between letting me make my own decisions and nudging me in productive directions. I credit Matt with introducing me to this field of research by allowing me to co-teach a secondary methods course. He has been instrumental in helping me think about, engage in, and share this work at state and national conferences. As a team, your support, encouragement, and constructive criticism helped me to stay the course. Thank you for your sustained attention to this project.

I would also like to thank my other committee members: Dr. Sarah Selmer, Dr. Melissa Luna, and Dr. Malayna Bernstein. I am grateful to have had the opportunity to learn from their expertise, both in relation to this study and throughout my doctoral program. I also appreciate the contributions of Neal Shambaugh, who served on my committee in the early stages of this research.

This dissertation has also been shaped in part by the other faculty members in the College of Education and Human Services (CEHS) from whom I have had the opportunity to learn, both through coursework and extracurricular workshops and events. I would also like to acknowledge the CEHS staff, particularly Connie Miranov, Char Allen, and Kim Goletz, for their assistance in scheduling courses, completing required paperwork, and managing grant funds.

I am grateful for financial support from the Wirt C. \& Mae S. Belcher Graduate Education Award that made it possible for me to purchase software subscriptions for myself and research participants, which allowed for a more efficient and robust analysis of data. I would also like to acknowledge support from Fairmont State University faculty members Carolyn Crislip- 
Tacy and Mike Ransom, who helped ensure that my research was approved by the Fairmont State Institutional Review Board.

This work would not have been possible without the participation of the four TCs from my methods class. Learning to teach is difficult work; participating in research while learning to teach even more difficult. Their questions and candid comments provided vital insights that will contribute to moving this field of research forward. I am also grateful for the mentor teachers and secondary students who were involved in this study. Since the ultimate goal of this work is to benefit secondary mathematics students, their participation is invaluable.

I would like to acknowledge the FSU Mathematics Department for their support throughout this process. I am especially grateful to Dennine LaRue for her moral support and for teaching my classes when I needed to be absent to fulfill doctoral requirements. I am also grateful to Joesph Riesen for regularly stopping by my office to ask about my progress; I always wanted to be sure I had something new to say, which kept pushing me forward.

I am thankful to all of my friends and family for their encouragement along this journey. My dissertation writing sessions with Tina Cowger were instrumental in helping me build the momentum to finish this degree. My mother-in-law, Pat Jones, helped me focus on the end goal by enthusiastically reminding me of graduation. My sisters, Heather Yoho and Kristin Hamilton, encouraged me with cards and texts and took on some of my responsibilities during especially busy writing times.

I am especially grateful for my parents, Steve and Karen Yoho, who taught me by example to be brave enough to set big goals and hard-working enough to achieve them. They have always encouraged and supported me. As a math teacher educator, if I can instill just a fraction of Mom's love of math teaching into teacher candidates, students will reap big benefits. 
To my husband, Ben, who celebrated every small victory and provided perspective after every setback: I do not know how I would have finished this without you. From driving me to long evenings of classes to preparing meals (and reminding me when it was time to eat them), your support has been unwavering. I'm not sure you knew what you were signing up for five years ago, but thanks for hanging in there with me. Looking forward to spending post-doctorate life with you.

Finally, I thank God for leading me to this opportunity and, through the support and guidance of everyone mentioned above, helping me to see it to completion. "Trust in the Lord and lean not on your own understanding. In all your ways, acknowledge Him, and He will direct your paths." (Proverbs 3:5-6) 


\section{Table of Contents}

Abstract ii

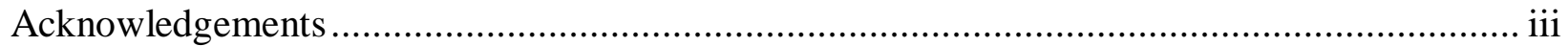

Table of Contents ................................................................................................ vi

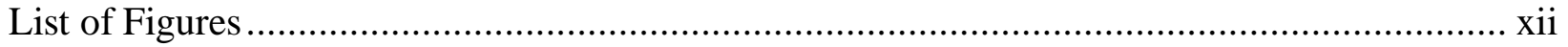

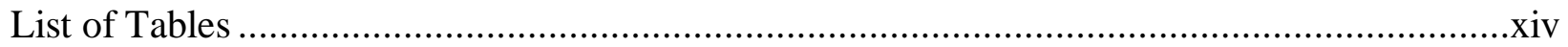

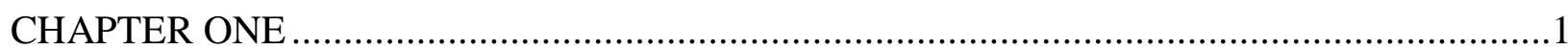

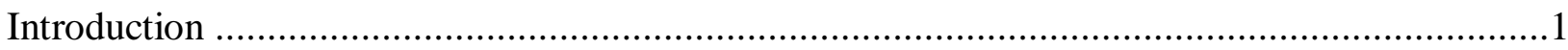

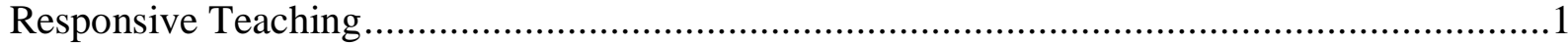

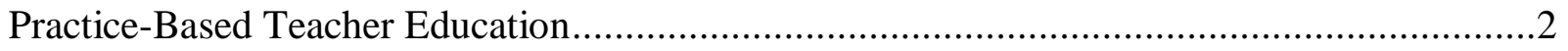

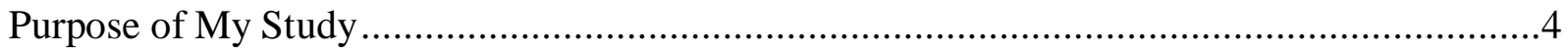

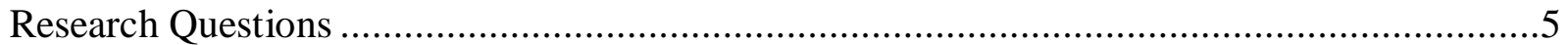

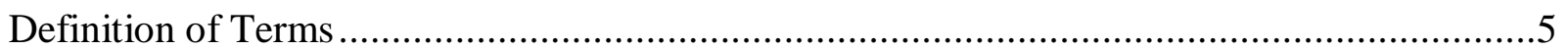

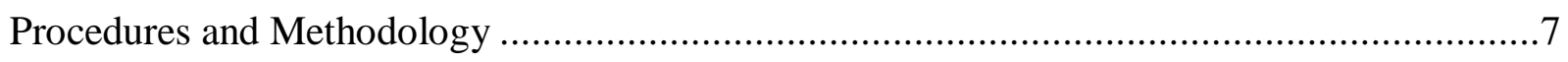

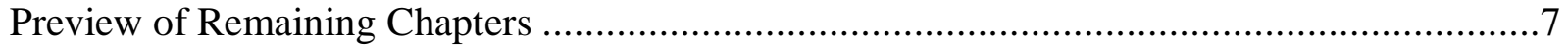

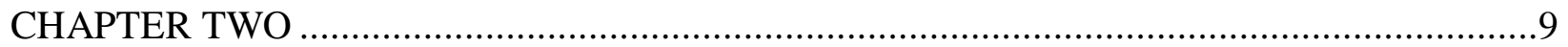

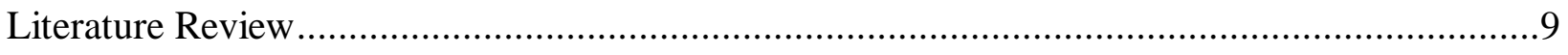

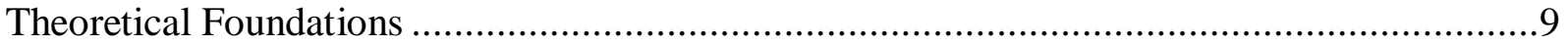

History of Teaching as a Practice........................................................................ 10

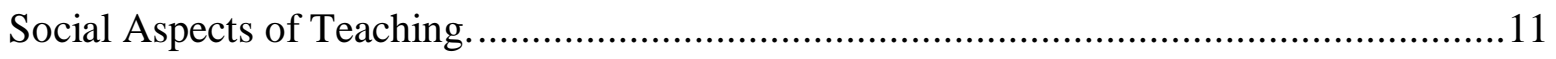

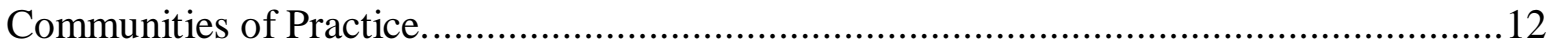

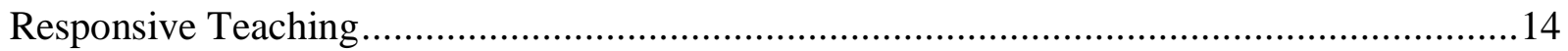

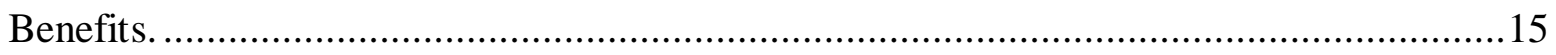

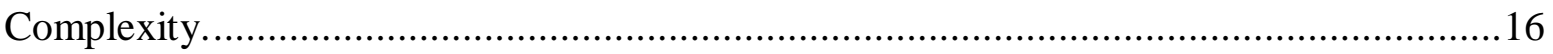

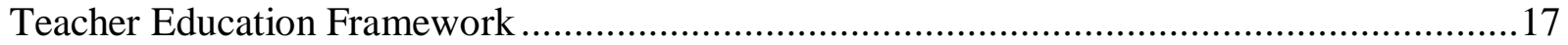

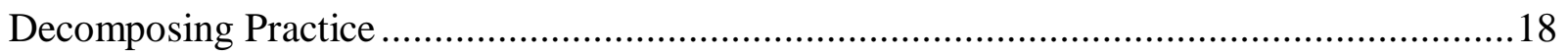

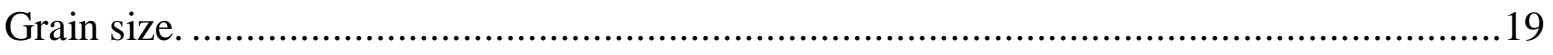

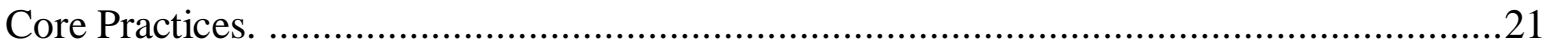

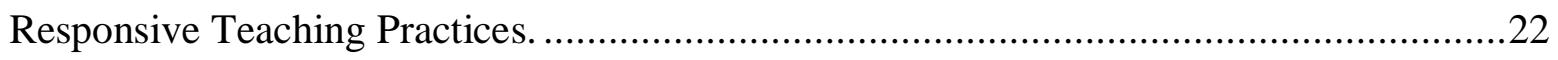

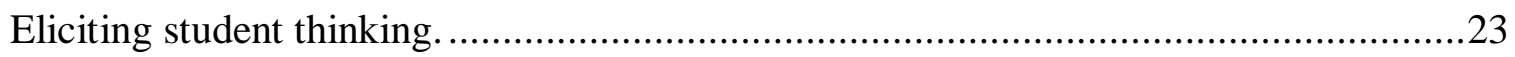

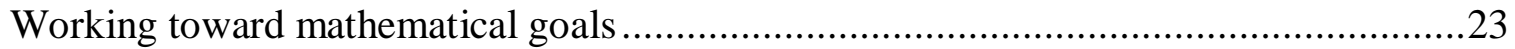




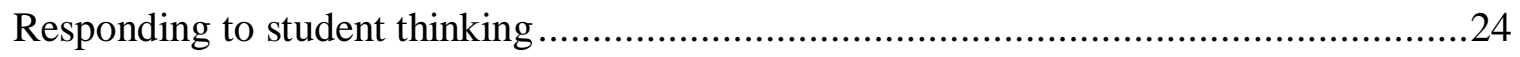

Orienting students to one another's thinking .......................................................25

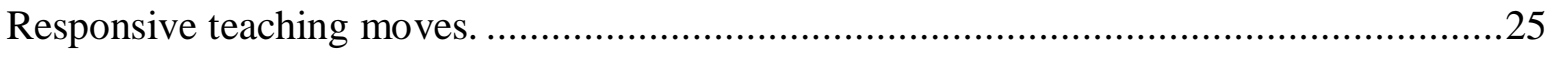

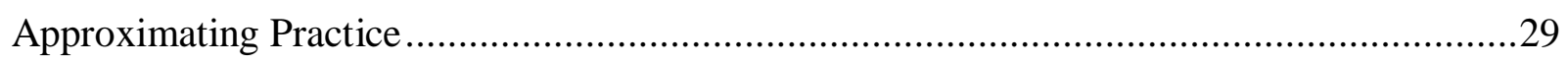

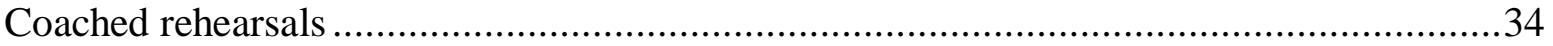

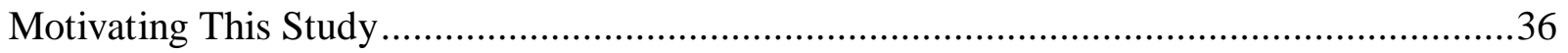

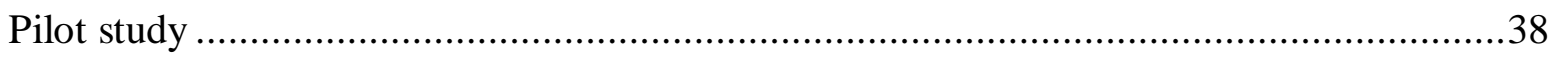

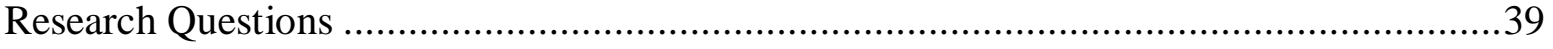

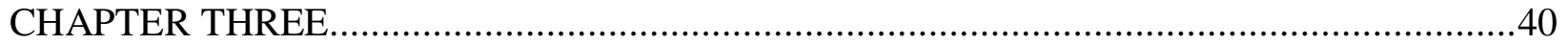

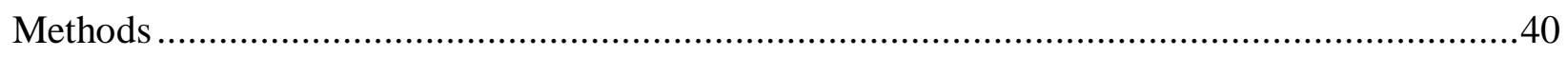

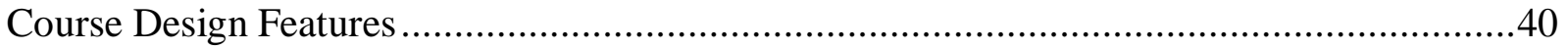

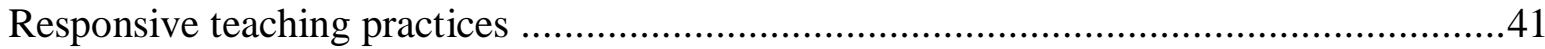

Adaptation of cycle of investigation and enactment. ............................................42

Implementation of cycle of investigation and enactment. ...........................................44

Secondary math education. ............................................................................ 45

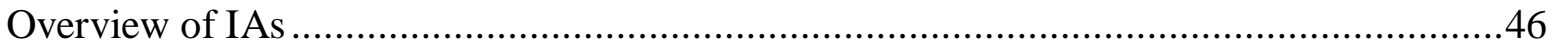

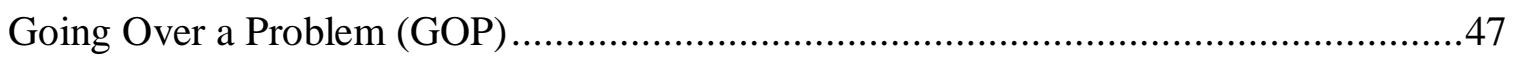

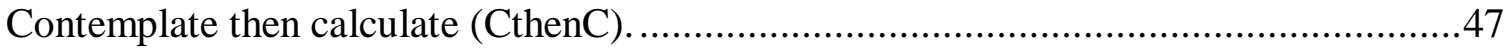

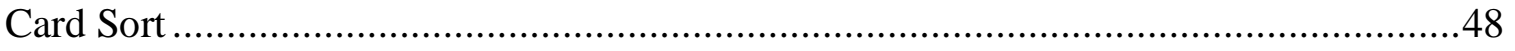

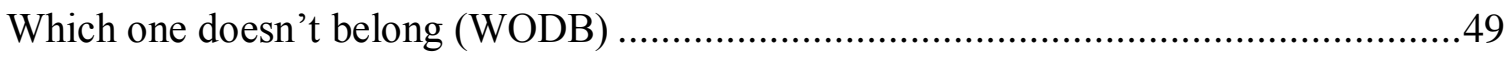

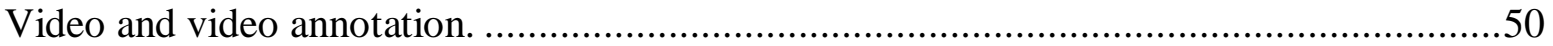

Research on the Implementation of the Methods Course Design ......................................51

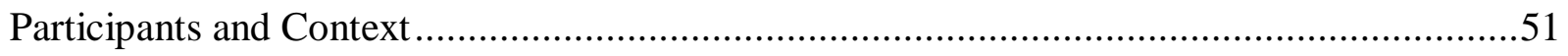

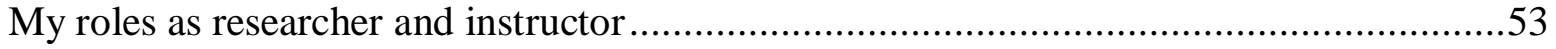

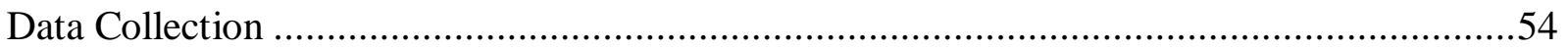

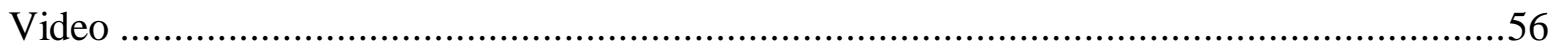

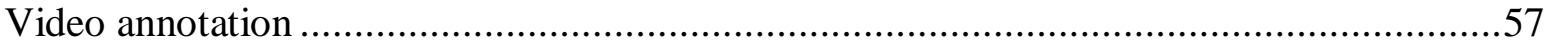

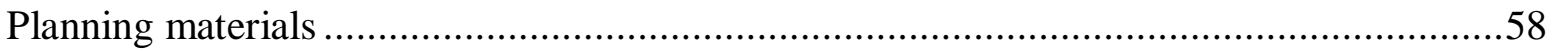

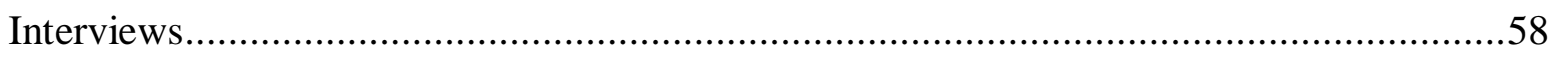

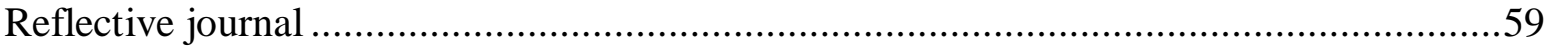




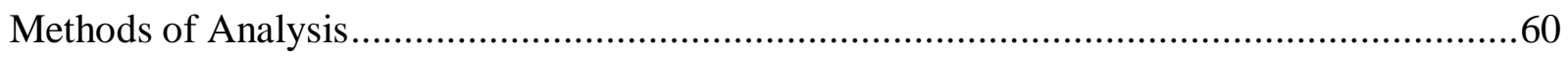

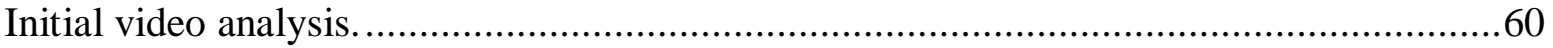

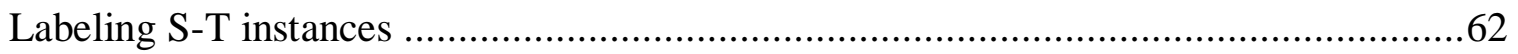

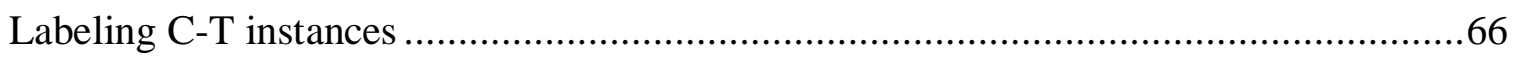

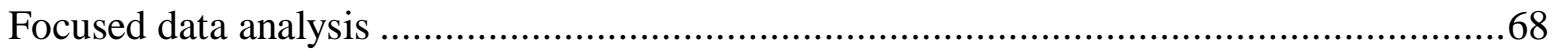

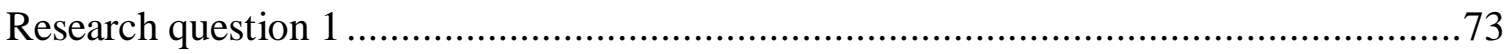

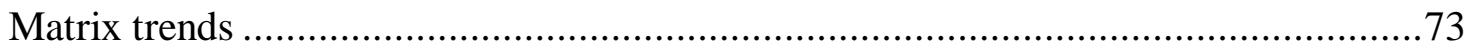

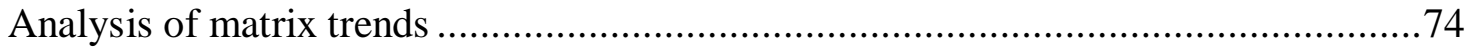

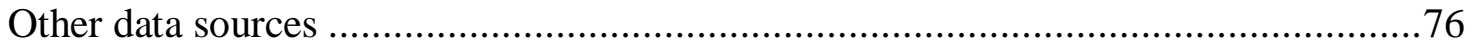

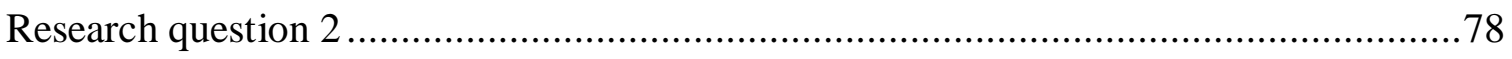

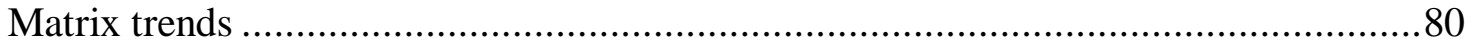

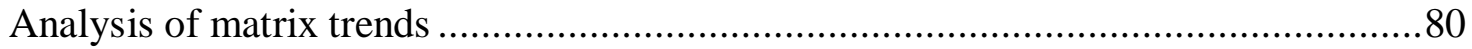

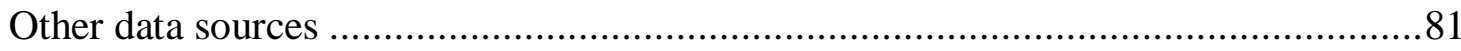

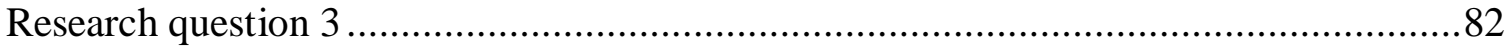

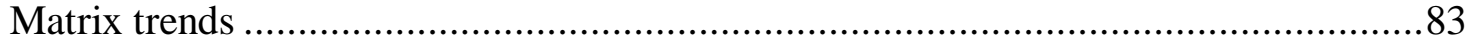

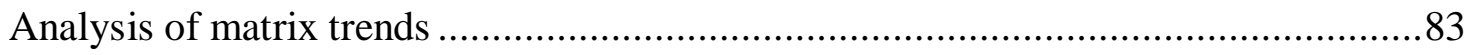

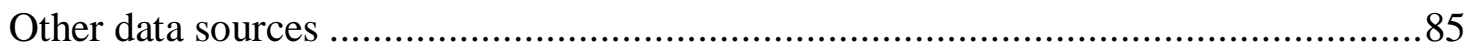

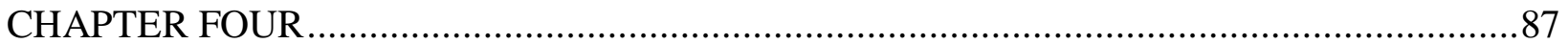

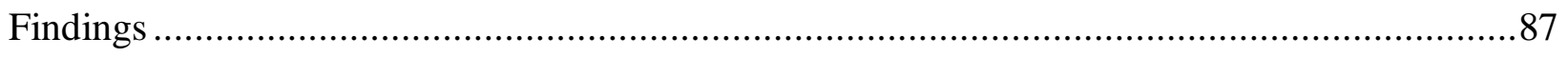

RQ1: How Do TCs Develop Skill in Teaching Mathematics Responsively Within a Methods Course in Which Cycles of Investigation and Enactment Are a Central Pedagogy?................88

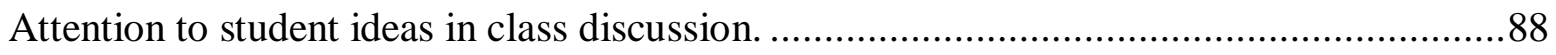

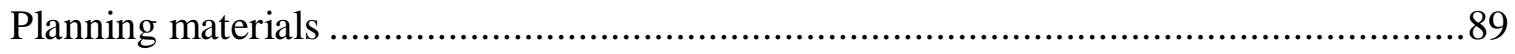

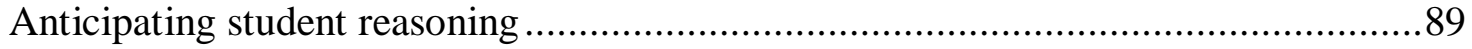

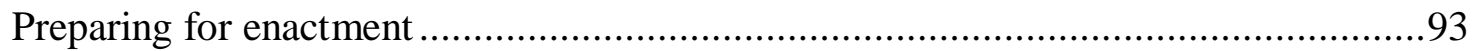

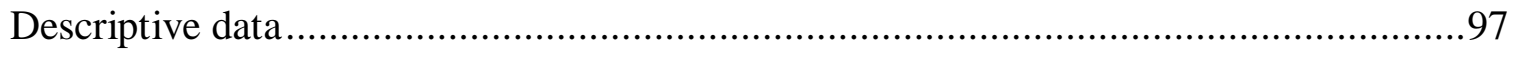

Responsive teaching moves enacted responsively .........................................................99

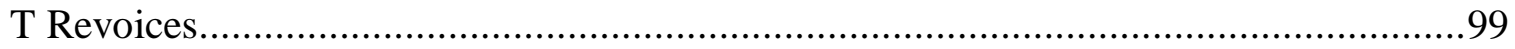

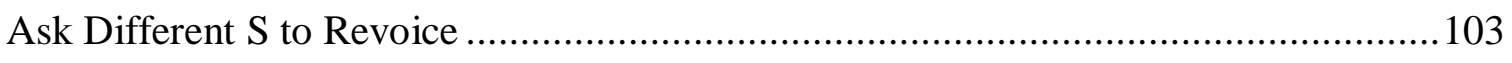

Comparing T Revoices and Ask Different S to Revoice …….......................................107 


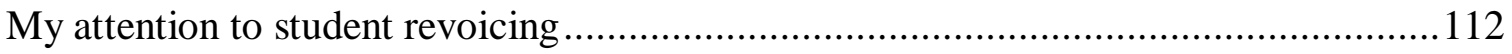

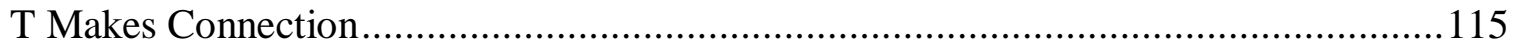

Potentially responsive moves not enacted responsively .......................................... 120

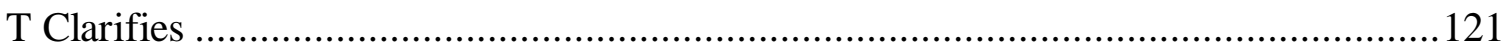

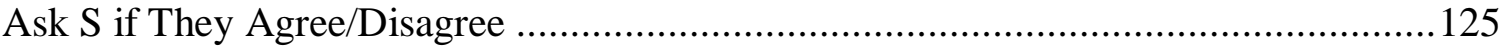

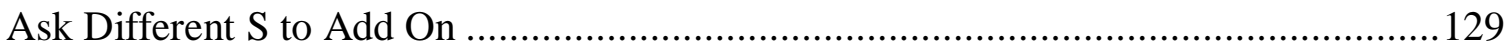

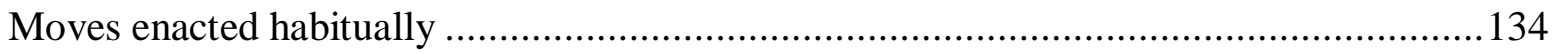

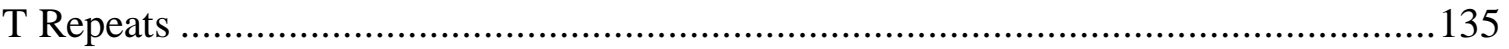

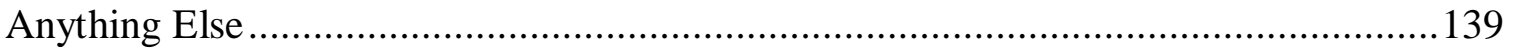

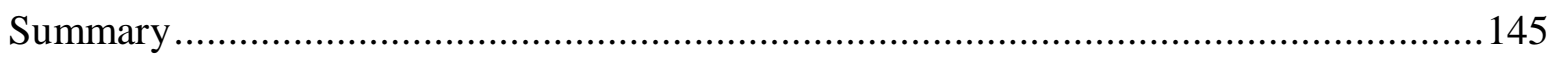

RQ2: How Does Coaching in Methods Affect TCs' Responsive Teaching? ........................ 145

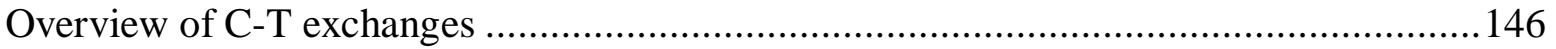

Comparing coaching interjections and TCs' enactments ....................................... 151

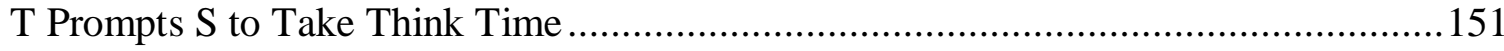

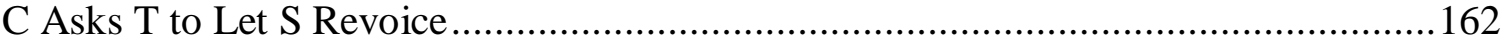

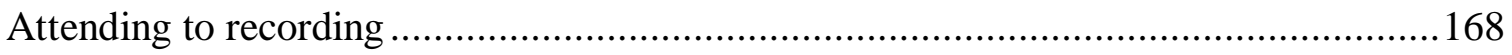

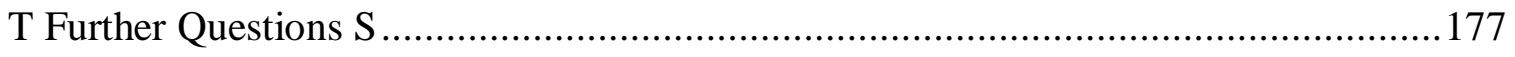

Interjecting to highlight underlying mathematics.......................................... 187

Interjecting to help clarify student thinking ............................................ 188

Coaching helped TCs' further question students responsively on their own.............190

C Asks T to Ask Open Questions ................................................................. 192

Responding to TCs' questions in the moment ................................................. 194

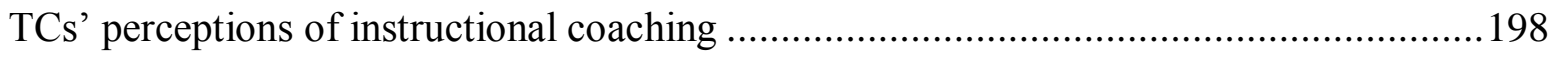

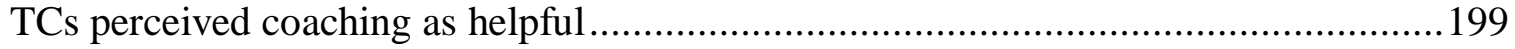

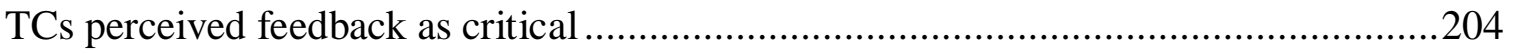

RQ3: To What Extent Do TCs Teach Responsively in School Settings? ...........................209

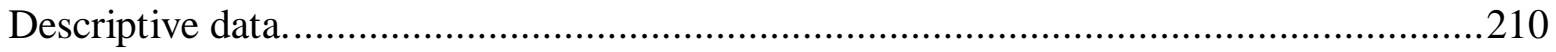

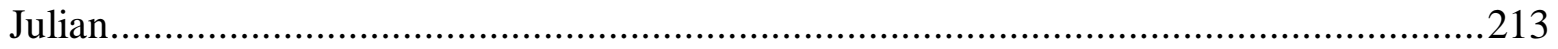

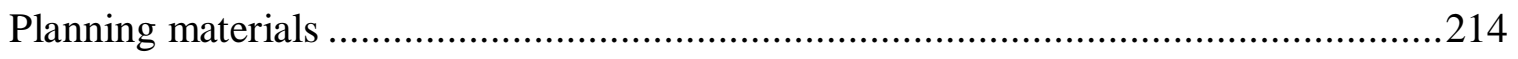

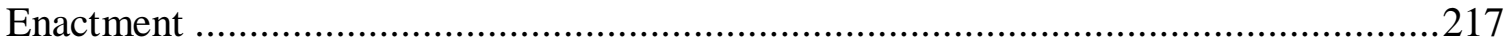




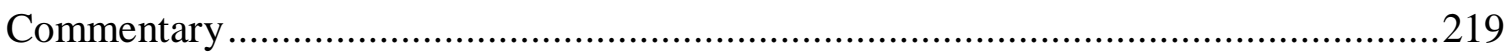

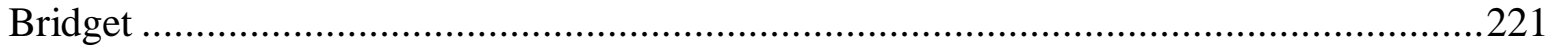

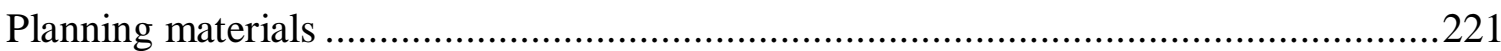

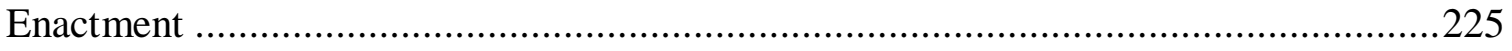

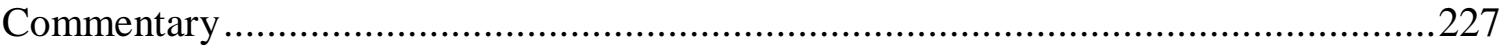

Grace

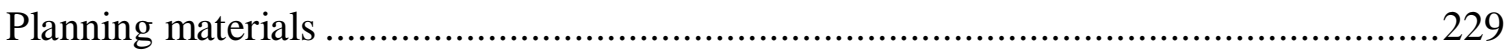

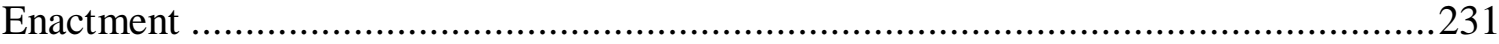

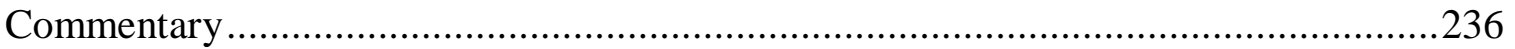

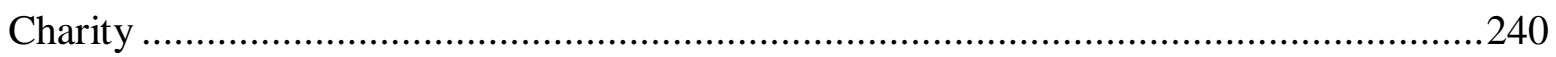

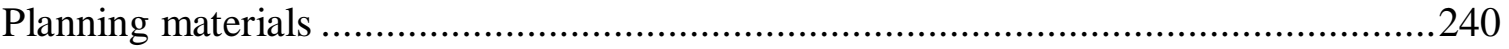

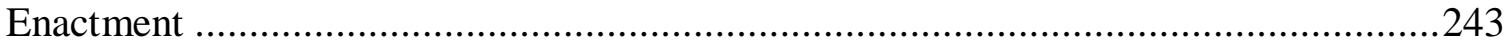

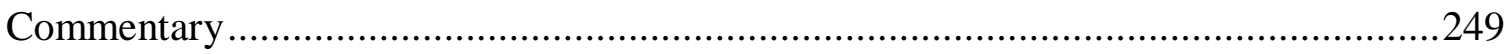

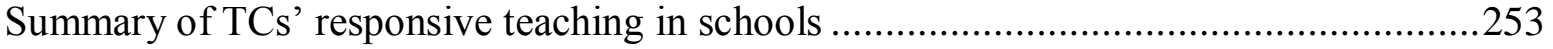

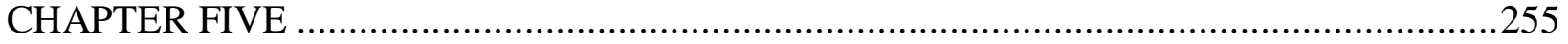

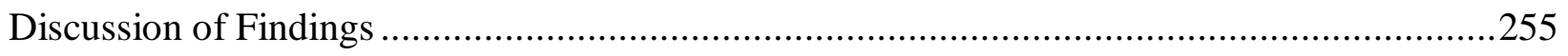

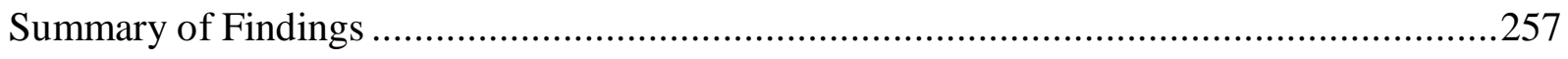

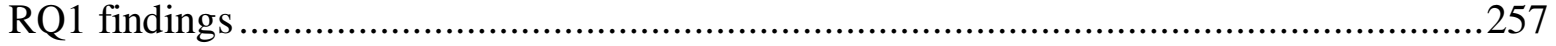

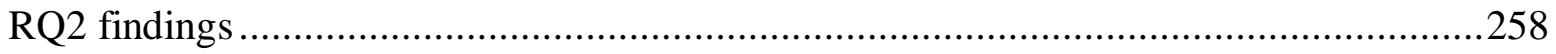

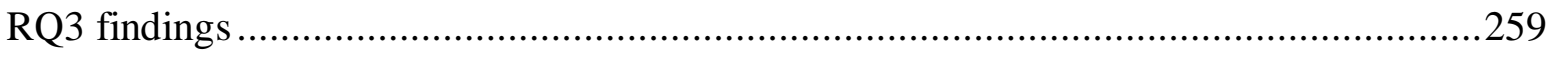

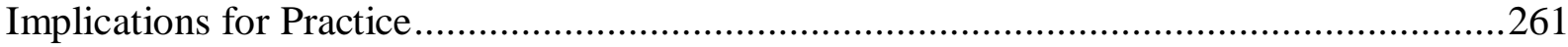

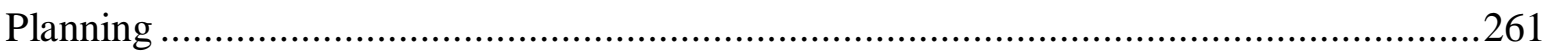

Potentially responsive moves not always enacted responsively ....................................262

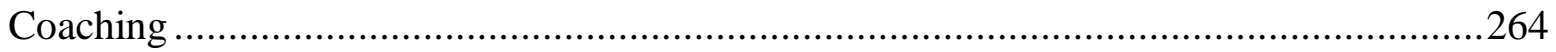

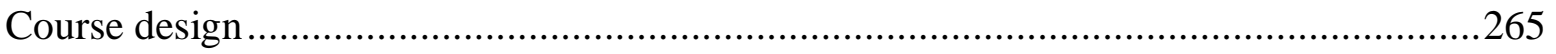

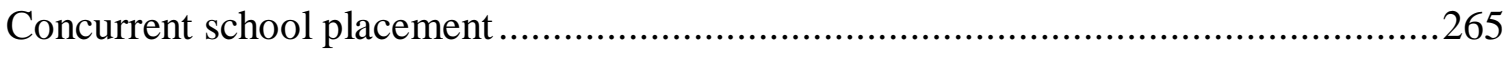

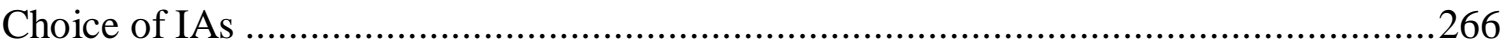

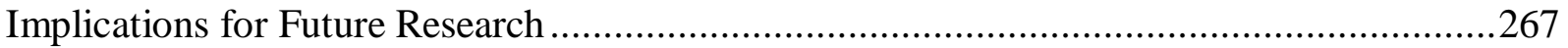

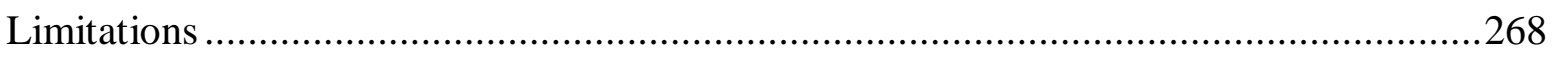

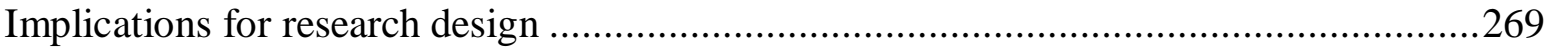




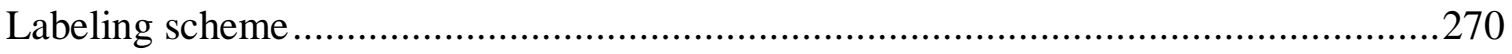

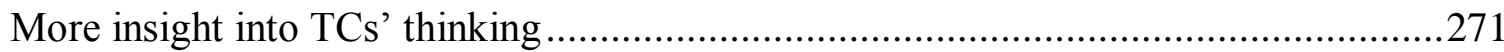

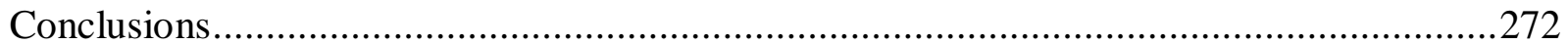

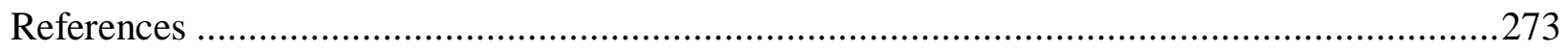

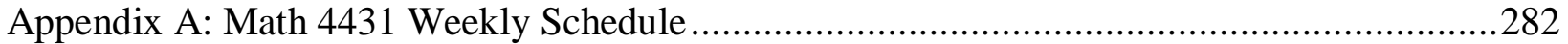

Appendix B: Planning Materials for Going Over a Problem .............................................283

Appendix C: Planning Materials for Contemplate then Calculate ......................................28

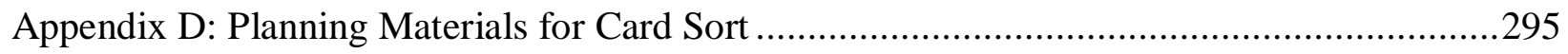

Appendix E: Planning Materials for Which One Doesn't Belong.......................................301

Appendix F: Prompts and Goals for Teaching Demonstrations and Rehearsals .....................306

Appendix G: Video Annotation Prompts ................................................................ 320

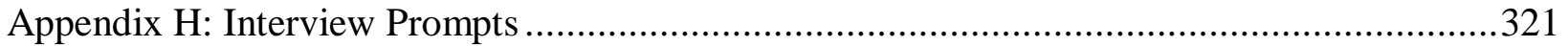




\section{List of Figures}

$\underline{\text { Figure }}$

$\underline{\text { Page }}$

1. Cycle for collectively learning to engage in an authentic and ambitious instructional activity ....

2. Cycle of Investigation and Enactment in Mathematics Methods Course

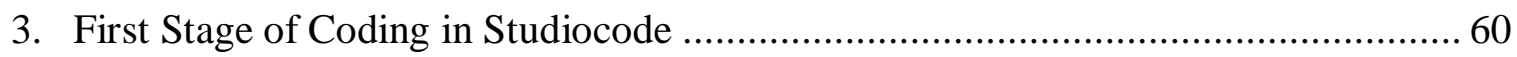

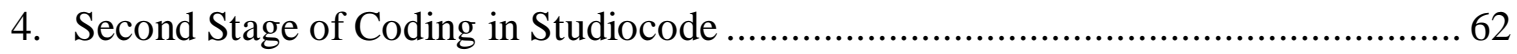

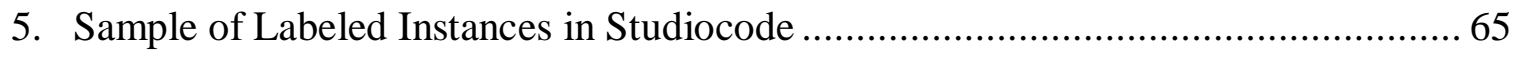

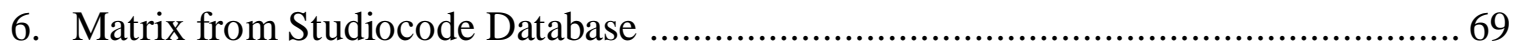

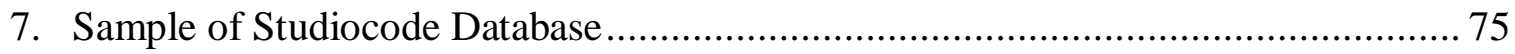

8. My Coding (Rows 1-6) and TC Video Annotations from Vosaic Connect (Rows 7-10)

9. Bridget's Pre-Planner for Going Over a Problem ............................................. 90

10. Bridget's Pre-Planner for Contemplate then Calculate ..................................... 92

11. Julian's notes about careful recording - Card Sort IA.......................................... 94

12. Charity's notes about careful recording - Card Sort IA (top), WODB IA (bottom)

13. Planning related to T Prompts $S$ to Take Think Time (Grace, CthenC; Charity, WODB).

14. Sample S-T Instances from Teaching Demo and WODB .................................... 98

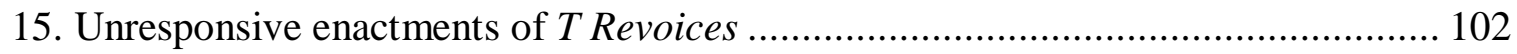

16. Reminders to Ask Different S to Revoice (Grace, CthenC; Charity, Card Sort) ....... 104

17. Responsive and Unresponsive Instances of Ask Different S to Revoice................... 106

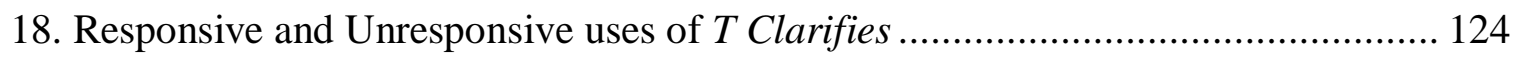

19. Responsive and Unresponsive Instances of Ask S if they Agree/Disagree .............. 128 
20. Two Types of Unresponsive Instances of Ask $S$ if they Agree/Disagree

21. Sample of Julian's video annotation comments for moments he labeled Support. 208

22. Julian's Problems and Goals for School GOP 214

23. Julian's Anticipated Student Responses for School GOP 216

24. Bridget's Anticipated Strategies for School GOP 222

25. Bridget's Problem and Goals for School GOP 223

26. Bridget's School GOP Protocol Notes 224

27. Grace's Problem and Goals for School GOP. 229

28. Grace's Anticipated Student Responses for School GOP 231

29. Grace's recording of student solutions during School GOP. 235

30. Charity's Problem for School GOP 241

31. Charity's Goals for School GOP 242

32. Charity's Anticipated Student Responses for School GOP 243 


\section{List of Tables}

Table

$\underline{\text { Page }}$

1. The Responding Framework (adapted from Milewski \& Strickland, 2016)............. 26

2. Research Questions, Data Sources, and Data Collection ................................... 55

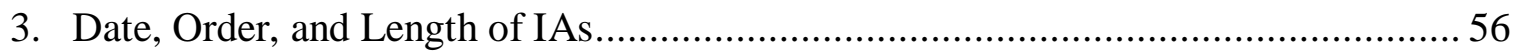

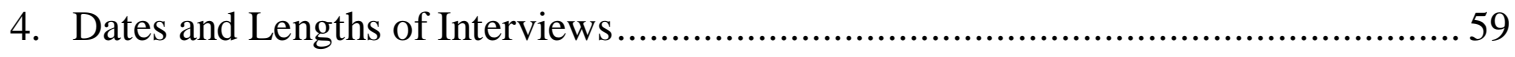

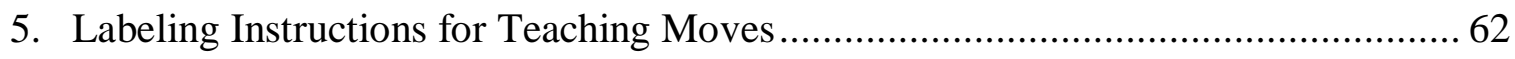

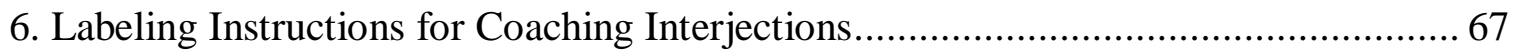

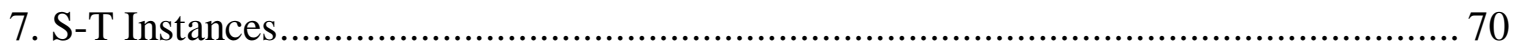

8. Research Questions, Data Sources, Methods of Analysis ..................................... 72

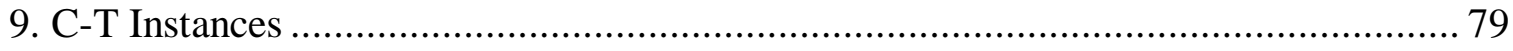

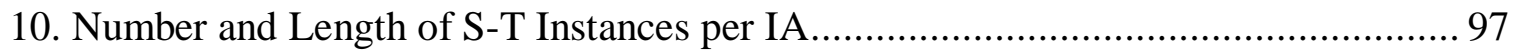

11. Responsive and Unresponsive Instances of T Revoices................................... 100

12. Responsive and Unresponsive Instances of Ask Different S to Revoice ................. 105

13. Comparison of responsive enactments of T Revoice and Ask S to Revoice............ 108

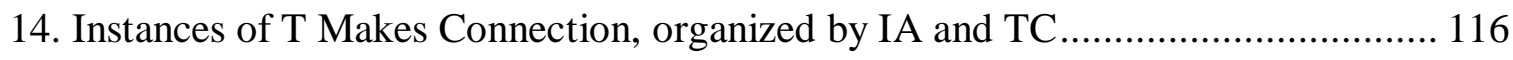

15. Responsive and Unresponsive Instances of T Clarifies .................................... 123

16. Responsive and Unresponsive Instances of Ask S if they Agree/Disagree............. 127

17. Responsive and Unresponsive Instances of Ask Different S to Add On ................ 131

18. Responsive and Unresponsive Instances of T Repeats .................................. 136

19. Responsive and Unresponsive Instances of Anything Else .............................. 142

20. Responsive and Unresponsive Substance Labels for C-T instances, organized by Structure. 
21. Instances of T Prompts S to Take Think Time (suggested in IA protocol vs. novel enactments).

22. Novel Instances of T Prompts $S$ to Take Think Time

23. T Prompts $\mathrm{S}$ to Take Think Time (responsive instances only) vs. C Asks $\mathrm{T}$ to Give Think Time

24. Ask Different $S$ to Revoice (responsive instances only) vs. C Asks T to Let $S$ Revoice

25. Responsive and Unresponsive Instances of $\mathrm{T}$ Records 170

26. T Records (responsive instances only) vs. C Comments on T's Recording (responsive instances only)

27. Types of Questions used in enactments of T Further Questions S (adapted from Boaler and Brodie, 2004)

28. Responsive and Unresponsive instances of Probing and Exploring Mathematical Meanings/Relationships questions (coaching not included)

29. TCs' Responsive and Unresponsive Enactments of T Further Questions S (organized by question type).

30. T Further Questions S (responsive only) vs. C Asks T to Further Question S 185

31. C Asks T to Further Question S, organized by question type 186

32. Instances of T Asks C a Question 195

33. Number and Length of S-T Instances per IA, including School GOP 210

34. TCs' responsive and unresponsive use of teaching moves during School GOP 212 


\section{CHAPTER ONE}

\section{Introduction}

The act of teaching is often perceived as universally straightforward—a person needs only to possess a skill in order to be qualified to teach someone else that skill. Consider tasks such as changing a tire, baking a cake, or riding a bicycle. In these cases, one only needs to find someone who can successfully complete the task themselves in order to learn how to do it. While these sorts of informal lessons are appropriately taught without any formal training, professional classroom teaching requires more than a teacher's ability to complete a task. In order for students to develop a deep understanding of a subject, such as mathematics, the teacher must not only have strong content knowledge, but also be able to explain the content and respond to questions in ways that are understood by the student. It is also important for the teacher to give students the opportunity to explore and grapple with new ideas on their own and with each other, rather than simply providing an explanation without giving students time to process them. Thus, making students' ideas central to classroom discussions is crucial to student learning.

\section{Responsive Teaching}

Focusing on students' ideas during class discussions requires teachers to adapt their instruction based on student thinking, a notion that has come to be known as responsive teaching (Hammer, Goldberg, \& Fargason, 2012; Jacobs, Empson, Krause, \& Pynes, 2015; Richards \& Elby, 2014). This type of teaching treats students as sense-makers and assumes that their ideas make productive contributions to classroom discourse (Kazemi, Ghousseini, Cunard, \& Turrou, 2015; Richards \& Robertson, 2016). Responsive teaching also encourages more equitable participation in the classroom, meaning that teachers attend to the contributions of culturally and/or socioeconomically diverse students (Brodie, 2011). In order for students to reap these 
benefits, however, responsive teaching demands that teachers not only have strong content knowledge and a belief that students are capable of making productive contributions to class discussions, but also the skills to carry out this interactive work.

The concern, then, should be around when (or if) teachers learn these skills. If they are developed only through experience, students who are taught by less experienced teachers are at a significant disadvantage when compared with their peers who are taught by more experienced teachers. If teacher candidates ${ }^{1}$ (TCs) were given opportunities to develop responsive teaching skills before entering the classroom, this development could be done in a university setting, under the guidance of a teacher educator (TE), rather than in a classroom of students. Helping TCs develop these skills before entering the classroom has the potential to provide more equitable learning experiences for all students.

\section{Practice-Based Teacher Education}

This suggestion, however, presents a challenge for teacher education programs: how should TEs, working in university methods courses, best prepare TCs to teach in school classrooms? The two settings are vastly different, which contributes to the difficulty TCs have in transferring what they have learned in the university setting into the public school setting (Ball \& Cohen, 1999; Grossman, Hammerness, \& McDonald, 2009). One way teacher education scholars and researchers have proposed to meet this challenge is to emphasize teaching as practice-based, rather than exclusively knowledge-based (Grossman, Hammerness, et al., 2009). However, in the limited amount of time afforded to TEs, there remains the question of exactly what should be central to university courses. What ideas will provide TCs with the farthest-reaching benefits of

\footnotetext{
${ }^{1}$ I use the term "teacher candidate" to refer to individuals who are enrolled in and progressing through a teacher education program.
} 
their university coursework? To this end, several researchers have begun to identify practices that they see as central to the work of teaching — what they call "high-leverage" or "core" practices for teacher education (Ball \& Forzani, 2011; Dotger, 2015; Forzani, 2014; Grossman, Hammerness, et al., 2009; McDonald, Kazemi, \& Kavanagh, 2013). A subset of these core practices are integral to the work of responsive teaching, such as eliciting and responding to student thinking and orienting students to one another's thinking (what I will refer to in this dissertation as "responsive teaching practices"). Identifying and focusing the work of teacher education on responsive teaching practices allows TEs a space in which to help TCs develop skills to enact these practices, in part through the enactment of teaching moves focused on responsive teaching. By introducing TCs to responsive teaching moves and helping them understand how to enact those moves in responsive ways, TEs can assist TCs in their development as responsive teachers.

Research on TCs' implementation of core practices and responsive teaching is still in its early stages. Some of the existing research focused on practice-based teacher education describes pedagogies of investigation (e.g., observation of teaching videos) and pedagogies of enactment (e.g., teaching a lesson) as productive ways to aid TCs in their development (Grossman \& McDonald, 2008). Some mathematics TEs have combined these two types of pedagogies to engage TCs in a cycle of investigation and enactment (McDonald et al., 2013). At the center of the cycle are instructional activities (IAs) that serve as "containers" for core practices, meaning that particular IAs allow TCs to focus on particular core practices (McDonald et al., 2013, p. 5). After experiencing the IA as students, TCs are then asked to participate in coached rehearsals of the IA. During these rehearsals, a single TC leads the IA while the other TCs act as students and the TE acts as a coach, pausing the rehearsal periodically to make suggestions or to highlight 
positive aspects of the TC's teaching. Finally, TCs reflect upon their enactments as a means of improving their skills for subsequent IA enactments. Following these reflections, the TE introduces a new IA and the cycle begins again.

\section{Purpose of My Study}

While these studies provide useful insights into how TEs might enact practice-based pedagogies in teacher education courses, more is to be known about how TCs develop skill in teaching responsively through the use of these pedagogies, especially with secondary mathematics TCs. This development involves more than merely enacting responsive teaching moves, but also considers whether TCs enact those moves for the purpose of responsive teaching and whether TCs execute teaching moves in responsive ways. A refined understanding of how TCs develop their abilities to enact responsive teaching would help TEs to select relevant responsive teaching practices as a central focus for methods courses to maximize TCs' development as responsive teachers. Being better able to focus on TCs' development as responsive teachers would also mean more teachers entering the classroom with the skills to engage student ideas, which ultimately has a positive impact on student learning.

It is also not clear from previous research how instructional coaching impacts TCs' development — what is it about in-the-moment coaching that helps support TCs' development as responsive teachers? Knowing more about what coaches can do to focus their feedback on responsive teaching, as well as how TCs receive and take up that feedback, will provide instructional coaches with valuable information as they aim to develop TCs' responsive teaching skills.

Finally, although the extent to which TCs enact core practices in university settings has been given some attention, it is still unclear whether they enact these practices in the classroom 
(and, if so, to what extent). A better understanding of which (if any) responsive teaching practices TCs transfer to the classroom setting will aid TEs in adjusting the focus of methods courses to better support TCs' development as responsive teachers. This may also lead mathematics education researchers to further study why TCs may favor some responsive teaching moves over others, which in turn would further aid TEs in designing methods courses which are more effective at developing TCs' responsive teaching skills.

\section{Research Questions}

In this study, I will investigate how TCs develop in their enactment of responsive teaching practices, both within a university methods course and between the methods course and their school placement in a mathematics classroom, through the collection and analysis of video of TCs' teaching and other artifacts, such as interviews and written reflections. More specifically, the following questions guided my study:

1. How do TCs develop skill in teaching mathematics responsively within a methods course in which cycles of investigation and enactment are a central pedagogy?

2. How does coaching in methods affect TCs' responsive teaching?

3. To what extent do TCs teach responsively in school settings?

\section{Definition of Terms}

1. Conceptual Tools - principles and frameworks which guide decisions about teaching (Grossman, Smagorinsky, \& Valencia, 1999)

2. Core Practices - practices that are central to the work of teaching, as determined by individual TEs or researchers (Ball \& Forzani, 2011; Dotger, 2015; Forzani, 2014; Grossman, Hammerness, et al., 2009; McDonald et al., 2013) 
3. Cycle of Investigation and Enactment - a teacher education design that allows TEs and TCs to investigate, enact, and reflect upon core practices through the use of IAs (McDonald et al., 2013); in the current study, TCs use this cycle to investigate, enact, and reflect upon responsive teaching practices

4. Instructional Activity (IA) - instructional activity designed to help TCs focus on particular core practices; at the center of the Cycle of Investigation and Enactment

5. Pedagogical Tools - tools used by TEs to aid TCs in their development as teachers; includes conceptual and practical tools

6. Practical Tools - strategies and resources which can be immediately used or enacted in classrooms (Grossman et al., 1999)

7. Responsive Teaching - occurs when teachers adapt instruction based on close attention to the substance of students' content-specific ideas (Richards \& Elby, 2014)

8. Responsive Teaching Moves - teaching moves with the potential to be enacted responsively (e.g., Ask Different $S$ to Revoice; Ask $S$ if they Agree/Disagree)

9. Responsive Teaching Practices - core practices that focus on responsive teaching (e.g., eliciting and responding to student thinking; orienting students to one another's ideas)

10. Teacher Candidates (TC) - individuals who are enrolled in and progressing through a teacher education program

11. Teacher Educators (TE) - individuals who prepare TCs for their work as teachers; in this dissertation, I primarily use this term to refer to university faculty who teach methods courses 


\section{Procedures and Methodology}

In this study, I draw upon previous methods course designs and research designs related to pedagogies of practice in an effort to understand how secondary mathematics TCs develop skill in responsive teaching across settings. During the methods course, each TC enacted a Teaching Demo and rehearsed a series of four IAs. Each IA rehearsal involved instructional coaching and was video recorded for TC reflection and for research purposes. Once the IA rehearsals in methods were completed, each TC enacted one IA in a school classroom. This enactment was also video recorded for TC reflection and research purposes. After each IA, TCs used video annotation software to identify and comment on what they saw as salient moments during their teaching. Throughout the semester, each TC also participated in three semistructured interviews to further discuss their teaching.

Data analysis for this study primarily took the form of video analysis. I watched TCs' teaching videos multiple times to identify instances of responsive and unresponsive teaching, based on their enactments of teaching moves. Data from their video annotations and interviews, along with my own reflective journal, provided further insights into TCs' reasoning behind their chosen use of certain teaching moves.

\section{Preview of Remaining Chapters}

This dissertation contains five chapters, references, and appendices. In Chapter 2, I review three main bodies of literature in an effort to situate my study at their intersection. First, I discuss research related to the historical foundations of teaching as a practice. Second, I discuss research on responsive teaching. Finally, I discuss research on practice-based teacher education with a focus on decomposing and approximating practice in mathematics teacher education to set 
the stage for Chapter 3, which discusses in detail the methods and procedures used to conduct my study.

In Chapter 4, I present my findings, organized around my research questions (RQs). Findings for RQ1 describe the kinds of moves that TCs enacted (responsively or unresponsively) during a methods course. Findings for RQ2 discuss how TCs' enactment of those moves was affected by instructional coaching. Findings for RQ3 are presented in four vignettes, which are meant to provide a glimpse into each TC's teaching in schools. Each vignette is followed by a commentary that compares each TC's teaching in methods and in schools. Chapter 5 contains my conclusions, implications for mathematics teacher education (including future iterations of this research), and recommendations for further research. 


\section{CHAPTER TWO}

\section{Literature Review}

In this chapter, I examine three bodies of literature that serve in framing and motivating this study. I begin by describing the theoretical foundations of practice-based teacher education - a discussion of the history of teaching as a practice, the social aspects of teaching, and the notion of communities of practice - to illustrate the complexity of learning to teach.

Next, I discuss literature around responsive teaching, highlighting its benefits for students as well as the challenges it presents to teachers. Finally, I describe a framework that may be used to aid TEs in designing practice-based methods courses with a focus on responsive teaching. I discuss two parts of this framework in detail: decomposing practice and approximating practice, as they relate directly to my research. I conclude this chapter with a section outlining the motivations for my study, including a brief description of a pilot study that informed my current work.

\section{Theoretical Foundations}

Teaching has been described in several ways: unnatural, intricate, and highly relational (Ball \& Forzani, 2009; Grossman, Hammerness, et al., 2009; Lampert, 2010). Such descriptions allude to the challenges of the work, but these are challenges that may be overcome. One can become more skilled at the work of teaching through, for example, developing skill in asking questions to which answers are already known, provoking disequilibrium, or probing students' ideas (Joyce \& Showers, 1981; Ball \& Forzani, 2009).

Prioritizing TCs' development of such skills will require teacher education programs to shift their pedagogy to focus more on teaching as professional practice (Ball \& Cohen, 1999). The notion of teaching as a "practice" could be conceived of in four different ways: (a) that which contrasts with theory, (b) one of several responsibilities involved in the work of teaching, 
(c) a rehearsal in preparation for future performance, or (d) a general term which describes engagement in professional work (e.g., an attorney practices law; Lampert, 2010). Throughout this dissertation, I will draw upon definitions (b), (c), and (d).

History of Teaching as a Practice. Throughout the early history of American education, teaching was considered a performance-oriented field. In the teacher preparation (normal) schools of the 1800s, TEs firmly believed that teachers needed to be trained for the work. As part of their training, TCs were required to observe expert teachers and emulate their lectures. In the early 1900s, the Commonwealth Teacher Training Study, conducted by Werrett W. Charters and Douglas Waples, identified over 1,000 tasks and activities performed by teachers. In the 1960s, a similar study, which identified hundreds of teacher tasks and activities, provided a basis for Competency-Based Teacher Education (CBTE). CBTE sought to organize teacher education around particular learning objectives (competencies) in self-paced programs (Forzani, 2014). It was around this same time that research related to the cognitive demands of teaching, such as teacher decision-making and teacher knowledge, came to the forefront (Grossman \& McDonald, 2008).

In the past 50 years, research on teaching has evolved from examining teacher characteristics (e.g., enthusiasm) to teacher content knowledge to, most recently, teacher reflection and dispositions (Grossman \& McDonald, 2008). During this time, methods courses were focused on helping TCs learn about good teaching rather than how to enact good teaching (Grossman, Hammerness, et al., 2009). Although shifts in teaching research led to shifts in teacher education programs, they were not sufficient; many TEs still identified a disconnection between novice teachers' theoretical knowledge and their practical work in the classroom. As a result, researchers began arguing for another shift in teacher education, one which again focused 
on teaching as a practice (Ball \& Forzani, 2009; Ball \& Cohen, 1999; Grossman, Hammerness, et al., 2009; Grossman \& McDonald, 2008; McDonald et al., 2013).

Current efforts toward educational reform share some similarities with the practice-based teacher education programs of the 1800s and early 1900s, but some significant differences as well. Consider, for example, the requirement of the normal schools that TCs imitate expert teachers' lectures, as though ideas need only to be transmitted to students in order for them to be learned (Forzani, 2014). In contrast, more recent research in teaching as a practice-based endeavor has viewed teaching as interactive work in which students' ideas take center stage (Ball \& Cohen, 1999; Brodie, 2011; Dyer \& Sherin, 2015; Jacobs, Lamb, \& Philipp, 2010; Lampert et al., 2013; Richards \& Elby, 2014). Thus, rather than asking TCs to merely imitate the actions of experienced teachers, TCs have been asked to develop skill in more "improvisational practices" (Forzani, 2014, p. 359). Improvisational work in the classroom involves incorporating students' ideas as part of instruction, which requires the teacher to make frequent in-the-moment decisions about which ideas to bring to the forefront and how to pursue them (Richards \& Elby, 2014; Thompson, et al., 2015). Examples of improvisational practices include eliciting, interpreting, and responding to student thinking, and facilitating class discussions (Forzani, 2014).

Social Aspects of Teaching. Recall that one way to conceive of the term practice is as a description of engagement in professional work, in the same way an attorney practices law (definition (d) above; Lampert, 2010). This most recent conception of teaching as a practice, with its focus on improvisation, takes into account more social aspects of that work by focusing on what teachers actually do to meet the demands of teaching (Grossman \& McDonald, 2008; Lampert, 2010). While more traditional cognitive theories focused on the practice of each individual teacher (or TC), sociocultural theory shifts the focus to the entire community of 
practice in which the TCs and TEs take part - a crucial shift if the field of teacher education is to focus on teaching as a practice-based, rather than a cognitive endeavor (Ghousseini, Beasley, \& Lord, 2015). Sociocultural theory also suggests that the settings in which TCs find themselves help to shape their understandings and practices (Grossman et al., 2000).

This suggestion, however, presents an additional challenge for teacher education programs: how should TEs, working in university methods courses, best prepare TCs to teach in school classrooms? The two settings are vastly different, which contributes to the difficulty TCs seem to have in transferring what they have learned in the university setting into the public school setting (Ball \& Cohen, 1999). They tend to fall victim to what Lortie (1975) called the “apprenticeship of observation," in which teachers draw more heavily upon their prior experiences as students than upon what they have learned in teacher education programs as they enter the field (as cited in Lampert, 2010, p. 30). In light of this challenge of transfer, sociocultural perspectives provide a useful lens through which to view teacher development. It allows for a focus on the multiple settings in which TCs learn to teach (e.g., university methods classrooms, public school classrooms) and how teachers carry out the work of teaching in those settings.

Communities of Practice. Teaching is inherently relational and interactive work. Developing the skills to manage that work can occur in both university methods classes and school classrooms. In a university methods course, TEs can provide TCs with opportunities to "teach" a content-specific lesson to their classmates, whereas in a school placement, TCs may have opportunities to teach children. Despite the fact that the "students" in a methods class are other TCs, as opposed to children in a school classroom, a focus on the interactive work of teaching in either setting provides an authentic context for learning about the improvisational 
work of teaching. The university methods course and the public school classroom each constitute a community of practice, where TCs have professional interactions about how to develop their teaching practice. Participation in communities of practice is important for TCs' development because it provides support as they learn about teaching as a practice (Ball \& Cohen, 1999).

In the community of practice that exists in the methods classroom, the TE acts as a knowledgeable other in that he or she is already a professional educator at the university level who may have public school teaching experience as well (Vygotsky, 1978). In that role, the TE may model teaching practices for TCs or provide feedback on TCs' instruction. Joyce and Showers (1981) note that simply learning a skill in a methods course does not mean it can or will easily be implemented in a school classroom. They argue that in order for the skill to transfer to another setting, TCs must learn to use the skills flexibly, know when to implement them, and know how to modify them based on the students in class.

TCs are also members of the community of practice that exists in the school clas sroom where they complete their fieldwork. In this case, the classroom teacher serves as the knowledgeable other, but often in a different sense than the TE. Classroom teachers are steeped in the day-to-day happenings of their students and the school community. The main objective of the teacher must be their own students; TC learning must be a secondary consideration (Haigh, 2007). While the primary focus in the methods course is to support TCs' learning to teach, the primary focus in a school classroom is the students' learning of the content, which may also involve classroom and behavior management strategies and the completion of tasks required by the school (e.g., taking attendance, hall duty). Therefore, TCs have different learning experiences in the two settings. Although it could be argued that all of these learning experiences are 
important for TCs' development, the learning in public schools often does not reinforce what TCs are learning in methods courses.

The relationships that exist between these two communities of practice constitute what Brofenbrenner (1979) characterized in his ecological model as a mesosystem. The TC is an active participant in both settings and each setting shapes the TC's conception of what it means to teach. Rosean and Florio-Ruane (2008) note that, traditionally, methods instructors have taken responsibility for helping TCs learn about the theoretical aspects (the why) of teaching, leaving the more practical aspects (the how) to TCs' fieldwork (as cited in Grossman, Hammerness, et al., 2009). However, TCs have struggled to enact the ideas they learned in their methods courses (Wideen, Mayer-Smith, \& Moon, 1998). For example, Kennedy (1999) describes a teacher education program in which TCs understood and agreed with the ideals put forth by the TE, but were frustrated to find that they were unable to translate the ideas into action to help their students achieve those ideals. Brofenbrenner's (1979) ecological model suggests that in order to determine whether TCs' development in practice has occurred, there must be evidence that activities and ideas learned in one setting are carried over into another. To ensure that TCs are applying what is learned in methods courses, they need support to understand how those ideas can be enacted.

\section{Responsive Teaching}

The notion of responsive teaching is helpful as TEs engage TCs in developing skills in managing the improvisational work of teaching. However, the term has been defined in multiple ways. Most generally, responsive teaching assumes that students are fully capable of learning and is critical of classroom norms that encourage the reproduction of knowledge, rather than the creation of knowledge, through classroom interactions (Thompson et al., 2015). The term is also 
used more specifically to refer to teaching in which instructional decisions are constantly adapted based on students' content-specific thinking (Hammer et al., 2012; Jacobs, et al., 2015). Richards and Robertson (2016) described the nature of responsive teaching as "(a) active and intentional, (b) highly contextualized, and (c) tension-filled" (p. 42). Despite these different definitions, all of these authors have noted the importance of making students' ideas central to the work of teaching. Zooming in on the work of responsive teaching a little further, it can be described as simultaneously: (a) attending to the substance of students' ideas with the goal of working to understand the student's point of view, rather than to evaluate it, (b) listening for connections between students' understanding and the discipline being studied, and (c) taking up and pursuing student thinking, making it central to instruction (Robertson, Atkins, Levin, \& Richards, 2016). I adopt the definition of "responsive teaching" proposed by Richards and Elby (2014), which states that responsive teaching occurs when teachers adapt instruction based on close attention to the substance of students' content-specific ideas.

Benefits. In mathematics education, a shift toward responsive teaching has been shown to account for similar levels of mathematical achievement among diverse groups of students. In a study of four high school classrooms, each of which contained students at different levels of mathematical achievement and socioeconomic status (SES), the mathematical gains made by the lowest performing class (primarily black students of low SES) matched the mathematical gains observed in the highest performing class (primarily white students of high SES). These gains were the result of the teachers in both classes shifting their practice to be more responsive (Brodie, 2011). Responsive teaching has been shown to enhance students' conceptual understanding and provide students with opportunities to engage in mathematical thinking and practices around important mathematical ideas (Hammer et al., 2012; Kazemi et al., 2015; 
Richards \& Robertson, 2016). Incorporating students' ideas in the classroom also provides a means for teachers to conduct formative assessment and push the boundaries of their own disciplinary knowledge and teaching practices. In turn, these shifts in teacher knowledge and practices affect the instructional decisions teachers make in the moment (Richards \& Robertson, 2016; Stahnke, Schueler, \& Roesken-Winter, 2016).

Complexity. The benefits of responsive teaching are not guaranteed or achieved without effort - this type of teaching is intellectually demanding. Jacobs, Lamp, and Philipp (2010) identify three interrelated skills (attending to children's strategies, interpreting their understandings, and using their understandings to decide how to respond) that must be carried out almost simultaneously as a teacher makes in-the-moment instructional decisions. Recognizing the potential in student ideas while keeping an eye toward the disciplinary definition or conception of the topic being discussed also requires that the teacher have a strong, flexible knowledge of the content (Robertson et al., 2016).

Further illustrating the demands of responsive teaching, recent work by Van Zoest, Peterson, Leatham, and Stockero (2016) considers the teaching practice of building upon student thinking, a subset of responsive teaching, which they conceptualize as "several teacher moves woven together to engage students in the intellectual work of making connections between ideas and abstracting mathematical concepts from consideration of their peers' mathematical thinking" (pp. 1284-1285). They identify two prerequisite moves and four sub-practices which are required for a teacher to successfully build on student thinking. Since teachers cannot fully anticipate which ideas students will put forward for consideration, all of these steps must occur in the moment, meaning that responsive teachers must display an increased level of improvisational skill. 
As a result of this complexity, the work of responsive teaching has created tension for teachers who sought ways to respect their discipline while also showing respect to children as capable thinkers (Ball, 1993). The choice to pursue students' ideas may lead the class away from the required curriculum, creating another type of tension. In the field of mathematics, responsive teaching demands that "teachers teach in response to what students do" while holding students accountable to mathematical learning goals (e.g., procedural fluency, adaptive reasoning; Kazemi, Franke, \& Lampert, 2009, p. 1). For example, at any given moment, mathematics teachers must decide whether to foreground mathematical facts and methods or the development of students' independent thinking skills (Richards \& Elby, 2014; Richards \& Robertson, 2016). Making these in-the-moment decisions adds to the complexity of responsive teaching (Jacobs et al., 2015; Stahnke et al., 2016). In fact, Robertson and colleagues (2016) state, "These 'instructional tensions' are at the heart of responsive teaching”' (p. 29).

Given the benefits of responsive teaching for students, but also the intellectual demands and the tension that are felt by teachers who aim to teach responsively, it is clear that TCs will require support in teacher education programs if they are to develop skill in enacting responsive teaching. Similarly, TEs will need support to productively enact pedagogies that will promote TCs' development as responsive teachers (Ball \& Cohen, 1999; Peercy \& Troyan, 2017).

\section{Teacher Education Framework}

Grossman, Compton, and colleagues (2009) proposed a framework for centering teacher education on practice by engaging TCs in representations, decompositions, and approximations of practice. They define representations of practice as the different ways practice is represented in professional education, decompositions of practice as breaking down practice into smaller parts for the purpose of teaching and learning, and approximations of practice as opportunities 
for novices to participate in practices that share at least some authenticity with the actual practices engaged in by professionals. They state that a challenge for professional education (or for any profession) is to identify and articulate what novices will learn in a university setting that could not be better learned in the actual context of practice (Grossman, Compton, et al., 2009).

Considering teacher education specifically, the first part of the framework, representing practice, involves making the work of teaching visible to TCs during methods courses. Such activities might include examining student work, reviewing lesson plans, or watching classroom videos of actual practice (Grossman, 2011). The second part, decomposing practice, involves delineating the complex work of teaching by considering its constituent parts to provide TCs with opportunities to analyze and enact particular teaching practices (Grossman, 2011; Grossman, Hammerness, et al., 2009). The third part of the framework, approximating practice, involves simplifying practice to allow TCs to focus on, enact, and develop particular aspects of teaching (Grossman, 2011; Grossman, Hammerness, et al., 2009). The latter two parts of the framework are discussed in more detail, as they relate directly to my research.

\section{Decomposing Practice}

Decomposing practice into simpler, smaller practices allows for the determination of which practices TCs should begin to develop in a methods course. This decision is not straightforward, in part because a common language does not exist to describe the work of teaching (Grossman, Compton, Igra, \& Williamson, 2009). This lack of a common language has hindered researchers and practitioners in the field from meaningfully discussing their efforts to move the field forward (Brodie, 2011; Grossman \& McDonald, 2008; Lampert, 2010; McDonald, et al., 2013; Milewski \& Strickland, 2016). As with the notion of responsive teaching discussed above, terms must be explicitly defined each time they are discussed. As multiple 
meanings are attributed to the same term, it becomes almost meaningless. (Consider, for example, the four different conceptions of the word "practice" mentioned above.) A common language would better enable TCs to access existing knowledge of teaching (Grossman \& McDonald, 2008). Additionally, it would enable TEs and researchers to discuss their work across contexts more readily. This communication would be particularly beneficial in distinguishing between teaching practices of small and large grain sizes, as each grain size affords different learning opportunities for TCs.

Grain size. Deciding upon an appropriate "grain size" of the practices to be learned (i.e., how detailed these practices should be) is another obstacle to decomposing the practice of teaching (Ball \& Forzani, 2011; Forzani, 2014). Forzani (2014) judged tasks such as choosing specific topics to be emphasized or adjusting the material to be taught within a time limit to be of especially fine grain size. In contrast, professional standards and resources tend to conceptualize teaching practices using a much larger grain size (e.g., assessing, planning, creating a mathematical learning environment; Boerst, Sleep, Ball, \& Bass, 2011; National Council of Teachers of Mathematics [NCTM], 2014). While a fine-grained decomposition of practice may benefit TCs by providing practices which can be rehearsed and refined, the specific nature of such tasks may leave TCs wondering why such specific tasks are important to the work of teaching (Boerst et al., 2011). Alternatively, employing a larger grain size aids TCs in learning the general structure and language of teaching, but it affords few opportunities to instruct TCs in how to successfully carry out such broadly defined practices (Boerst et al., 2011; Sleep, 2012).

Grossman and colleagues (1999) provide another means of helping TCs understand the why and the how of teaching practices. They identified two types of what they term "pedagogical tools" (p. 13), which aid teachers as they construct and enact teaching practices. The first type, 
conceptual tools, are more abstract in nature and include principles and frameworks which guide decisions about teaching. They do not provide specific actions to help solve problems that arise in teaching, but rather provide some structure with which to think about teaching, which helps to explain why certain practices are beneficial to students. The second type, practical tools, does include strategies and resources that can be immediately used or enacted in classrooms, which helps to explain how to successfully carry out the practices. Some researchers and TEs are utilizing the notion of conceptual and practical tools to encourage TCs to use what they are learning in methods courses once they are teaching in schools. Both types of pedagogical tools can be introduced and developed in a methods class, but they are also designed to have applicability in a wide variety of school classrooms (Lampert et al., 2013; Kazemi et al., 2015).

In an effort to balance the why and the how of teaching practices for their TCs, Boerst and colleagues (2011) introduced TCs to teaching practices of varying grain size throughout the semester. They found that first introducing TCs to practices of a smaller grain size and then gradually working students up to enacting practices of a larger grain size afforded them opportunities to help TCs see how the different practices were related to one another. Their work was centered on the domain of leading a mathematical discussion. Therefore, all of the teaching practices that were enacted in the methods course, whether the grain size was small or large, supported TCs in learning to lead a mathematical discussion. This approach also allowed the TEs to support TCs in both the how and the why of teaching practice (Boerst et al., 2011; Ghousseini \& Herbst, 2014). It is the responsibility of the TE to decide which practices are most important for TCs to learn in a university setting while also attending to the affordances and limitations inherent in varying grain sizes. 
Core Practices. Shifting the focus of teacher education to be more practice-oriented requires the identification of practices (the responsibilities involved in the work of teaching; Lampert, 2010) that are central to the work of professional teaching. To this end, as previously stated, several researchers have begun to decompose the practice of teaching into smaller practices that they see as central to the work of teaching — what they call "high-leverage" or "core" practices for teacher education (Ball \& Forzani, 2011; Dotger, 2015; Forzani, 2014; Grossman, Hammerness, et al., 2009; McDonald et al., 2013). These are defined as teaching practices that TCs can begin to develop in a university setting as a means of shifting the pedagogy of teacher education (Ball \& Forzani, 2011; McDonald, et al., 2013). Core practices should be grounded in research, preserve the complexity of teaching, and involve actions or tasks that occur frequently in classrooms, regardless of the grade or ability level of their students. Practices that are identified as high-leverage should also be practices that TCs can actually begin to master in a methods course and that can appropriately be enacted in K-12 classrooms, thus allowing TCs to continue to develop these practices during their field experiences (Grossman, Hammerness, et al., 2009).

Some core practices, such as planning and evaluating students' work, are central to the work of teaching but are not interactive (Ball \& Forzani, 2009). Other core practices, such as eliciting and responding to student thinking, orienting students to one another's ideas, and facilitating a classroom discussion, focus more on the interactive aspects of teaching (Lampert et al., 2013; Kazemi et al., 2015; Baldinger, Selling \& Virmani, 2016). These types of classroom interactions become even more complex when working toward the goals of responsive teaching, in that the ways teachers choose to respond to student thinking or orient students to one another's ideas should foreground student thinking while also pushing toward a mathematical goal. 
Responsive Teaching Practices. While some researchers work toward a set of core practices that span a broad range of grade levels and disciplines (Ball \& Forzani, 2011; Dotger, 2015; Grossman, Hammerness, et al., 2009; McDonald et al., 2013), others are identifying core practices that are discipline-specific. Mathematics TEs face a specific set of challenges related to shifting to a practice-based view of teacher education. In one of their six principles of school mathematics, The National Council of Teachers of Mathematics (NCTM) states that teaching mathematics involves ensuring that teachers understand what students already know, what they need to learn, and what techniques to use to challenge and encourage students to meet learning goals (NCTM, 2000). To successfully gain an understanding of what students know, teachers must be able to elicit and use student thinking as a means of assessing students' progress toward mathematical goals and adjusting instruction to support student learning (NCTM, 2014). However, this is a skill that has not been given widespread attention in traditional education programs.

Research has shown that rigor is elevated when students' ideas and reasoning are made public during a classroom discussion that remains focused on the goal(s) of the lesson (Thompson et al., 2015). Thus, it is critical that TCs elicit and respond to student thinking and orient students to one another's ideas while simultaneously attending to the mathematical goal(s). This study will consider the practices of eliciting and responding to children's mathematical thinking and orienting students to one another's thinking, both in whole-class discussions. I term these responsive teaching practices and will examine these practices as they relate to responsive teaching. In the sections that follow, I discuss research related to eliciting student thinking, working toward mathematical goals, responding to student thinking, and orienting students to one another's thinking 
Eliciting student thinking. Responsive teaching may be seen in a variety of classroom interactions, but in mathematics education, one of the most common is eliciting and responding to student thinking (Franke et al., 2009; Lampert et al., 2013). Eliciting student thinking is included as one of the high-leverage practices identified by NCTM (2014). One thing teachers can do to elicit students' mathematical thinking is to choose high-level tasks that require students to represent and justify their mathematical understanding (NCTM, 2014). Teachers can also write questions before a class discussion that will allow for the diagnosis of conceptual gaps or common errors (NCTM, 2014). The act of eliciting students' ideas has been shown to be complex, as it often involves posing an initial question and then pressing students for more detailed explanations of their work (Jacobs et al., 2015).

Working toward mathematical goals. After student thinking has been elicited, but before the teacher responds, the teacher must decide to what extent the student's idea should be pursued. If a teacher follows every student contribution with a question such as, "Can you say more about that?", already limited class time may be lost in explanations of student work that do not bring the class closer to achieving the mathematical goal of the lesson (Leatham, Peterson, Stockero, \& Van Zoest, 2015). The instructional decisions that take place between eliciting and responding to student thinking depend heavily upon what the teacher notices about students' comments (Colestock \& Sherin, 2016; Jacobs et al., 2010).

In mathematics education, Leatham and colleagues (2015) have identified classroom instances that they term "Mathematically Significant Pedagogical Opportunities to Build on Student Thinking," or MOSTs. MOSTs must first be grounded in student mathematical thinking, then prove to be mathematically significant (developmentally appropriate and central to the mathematical goals), and finally, must afford a pedagogical opportunity. These researchers 
conceptualize a pedagogical opportunity as an instance when student thinking allows the teacher to act in a way that builds upon student thinking to move the class toward a mathematical goal, a crucial part of supporting students' learning of mathematics (Leatham et al., 2015; Sleep, 2012). Their work is focused on the teaching practice of recognizing (not responding to) "potentially productive student thinking once it has occurred" and on when it is productive to act on student thinking in the moment it occurs (Leatham et al., 2015, p. 90). The MOST construct allows teachers and researchers to view classroom interactions through a lens that focuses on why a teacher might have chosen to respond in a particular way, rather than how they actually responded.

In her work focused on steering instruction toward the mathematical point, Sleep (2012) notes that "to support beginners in learning how to enact the whole it is necessary to articulate its parts" (p. 964). She decomposed the practice of teaching toward a mathematical goal by articulating seven central tasks that are involved in that practice (e.g., spending instructional time on the intended mathematics, making sure students are doing the mathematical work). Sleep (2012) recognizes that steering toward a mathematical point requires teachers to manage multiple purposes simultaneously. Thus, the ability to foreground the mathematical goal while still managing nonmathematical purposes largely determines how successful the teacher is at maintaining a focus on the mathematical point of the lesson.

Responding to student thinking. While a consideration of which instances of student thinking are worth pursuing is a crucial part of the classroom interaction, teachers must also decide how they will respond once a student has vocalized an idea and then successfully carry out that response. There are numerous ways to respond to student thinking once a problem has been solved correctly, but teachers' responses have often involved only a few, usually evaluative 
comments about whether the student's contribution is correct or incorrect (Milewski \& Strickland, 2016). Teachers who participated in professional development related to students' mathematical thinking consistently asked students to explain their thinking, but showed great variability in the ways in which they responded to student explanations (Franke et al., 2009).

Orienting students to one another's thinking. Teachers must not only consider how they will respond to students' ideas, but also how they might help students engage with one another's ideas. Orienting students to one another's ideas is an ambitious teaching practice and an important aspect of facilitating class discussions (Baldinger et al.,2016; Kazemi et al., 2015; Lampert et al., 2013). Teachers in a study conducted by McDonald and colleagues (2013) described uncertainty about how to draw other students into mathematical conversations, which led them to identify orienting students to one another's ideas as a core practice of teaching. Teachers must help students must learn to engage ideas that originate with other students in order to ensure the involvement of all students in class discussions.

Responsive teaching moves. As core practices, eliciting and responding to students' mathematical thinking and orienting students to one another's thinking provide ways to articulate core and integral aspects of the work teachers do in their interactions with students and mathematics. However, as Boerst and his colleagues (2011) assert, the larger grain size of such practices does not necessarily provide the specifics for how a teacher can successfully carry out the work to be accomplished. Thus, while the decomposition of practice and identification of core practices serve as tools to mediate teachers' work, they serve more as conceptual tools that provide a language for the work of teaching, though is ultimately still abstract (Grossman et al., 1999). Thus, teachers also need more practical tools that support carrying out the work of teaching. As such, I focus on teaching moves as part of a focus on teaching practice. 
A teaching move is defined as something a teacher says or does during instruction (Boerst et al., 2011). Thus, I define a responsive teaching move as characterized by a comment or action from the teacher that makes student reasoning central to instruction. Researchers have identified a variety of responsive teaching moves. A responding framework, which identified fourteen possible response moves, was created by a group of secondary mathematics and science teachers in an effort to expand their repertoire of response moves and to address the need for a common language among researchers and practitioners (Milewski \& Strickland, 2016). This framework has been used by researchers to help identify shifts in teachers' practices of responding. An adapted framework may be found in Table 1. (These adaptations are explained in Chapter 3.)

Table 1

The Responding Framework (adapted from Milewski \& Strickland, 2016)

\begin{tabular}{lll}
\hline \multicolumn{1}{c}{ Move } & \multicolumn{1}{c}{ Description } & \multicolumn{1}{c}{ Example Teacher Move } \\
\hline Evaluate & $\begin{array}{l}\text { Assessing a student's idea as correct } \\
\text { or incorrect. }\end{array}$ & Good thinking. \\
Elicit Strategy & $\begin{array}{l}\text { Probing or clarifying questions that } \\
\text { get at student understanding. }\end{array}$ & $\begin{array}{l}\text { Walk us through your steps. How } \\
\text { did you begin? }\end{array}$ \\
Teacher Revoice & $\begin{array}{l}\text { Restating or paraphrasing a student's } \\
\text { ideas. Sometimes used to clarify, } \\
\text { check for understanding, or amplify } \\
\text { the student's idea. }\end{array}$ & So what I hear you saying is... \\
& $\begin{array}{l}\text { Inviting students to interact with } \\
\text { another student's idea in any of the } \\
\text { following ways: }\end{array}$ & \\
Invitation & $\begin{array}{l}\text { Inviting students to make a revoicing } \\
\text { move. }\end{array}$ & $\begin{array}{l}\text { Can anyone restate what Caleb } \\
\text { Ask Student to }\end{array}$ \\
\hline
\end{tabular}




\begin{tabular}{|c|c|c|}
\hline Move & Description & Example Teacher Move \\
\hline $\begin{array}{l}\text { Ask Student to } \\
\text { Connect }\end{array}$ & $\begin{array}{l}\text { Inviting students to make a connecting } \\
\text { move. }\end{array}$ & $\begin{array}{l}\text { How is Nick's idea related to } \\
\text { ideas from the last chapter? }\end{array}$ \\
\hline $\begin{array}{l}\text { Ask Student to } \\
\text { Add On }\end{array}$ & Inviting students to add on. & $\begin{array}{l}\text { Does anyone have anything to } \\
\text { add on to Charlie's idea? }\end{array}$ \\
\hline $\begin{array}{l}\text { Ask Student } \\
\text { If They } \\
\text { Agree/Disagree }\end{array}$ & $\begin{array}{l}\text { Asking students to apply their } \\
\text { reasoning to someone else's reasoning }\end{array}$ & $\begin{array}{l}\text { How many of you agree with } \\
\text { Cruz? }\end{array}$ \\
\hline Ignore & $\begin{array}{l}\text { Teacher does not address idea. It is } \\
\text { usually followed by an elicitation for } \\
\text { another idea without coming back to } \\
\text { original idea. }\end{array}$ & $\begin{array}{l}\text { Okay, does anyone else have } \\
\text { another idea? }\end{array}$ \\
\hline $\begin{array}{l}\text { Teacher Makes } \\
\text { Connection }\end{array}$ & $\begin{array}{l}\text { Pointing students to recognize the } \\
\text { relatedness of one's student's ideas to } \\
\text { another idea previously discussed or } \\
\text { known. }\end{array}$ & $\begin{array}{l}\text { Joe's method is similar to Ann's } \\
\text { because they both use what they } \\
\text { notice about the table to develop } \\
\text { an equation. }\end{array}$ \\
\hline
\end{tabular}

Enacting responsive teaching moves is about more than simply asking particular questions (e.g., Do you agree?). Teachers must identify moments when enacting responsive teaching moves will be beneficial, and then enact the moves in responsive ways. Thus, as TCs develop skill in enacting responsive teaching moves, they must not only understand why a particular responsive teaching move is beneficial to students in a particular moment, but also how to enact the move in responsive ways. As TCs develop flexibility with these whys and hows of teaching, they should also be able to articulate why a particular move was useful at a particular time and toward a particular purpose.

These teaching moves are often dependent upon the content being discussed (Robertson et al., 2016). For example, while a responsive teaching move such as revoicing another student's idea is likely to be beneficial across disciplines, a move such as comparing strategies is likely to 
be most useful in mathematics. Thus, the ways in which teachers choose to respond to particular aspects of student thinking, while leaving others unchallenged, have been (perhaps subconsciously) informed by a consideration of the norms of the discipline being studied (Richards \& Elby, 2014; Thompson et al., 2015). These "twin imperatives of responsiveness and responsibility" may create feelings of tension for teachers (Ball, 1993; p. 374), which further underscores the fact that developing skill in the enactment of responsive teaching moves is no easy task.

As an example, in a study of high school math teachers' responsive teaching practice, Dyer and Sherin (2015) noted that when a student's idea was unexpected, even experienced teachers responded by looking for a relationship between the structure of the mathematical task (i.e., the numbers given) and the mathematics in student thinking. The authors posited that the level of experience held by the teachers in their study allowed them to more easily make connections among different students' ideas. This implies that teachers with less experience (TCs) would be even more likely to look to the structure of a mathematical task in order to make sense of a student's unexpected contribution, rather than making connections among students' ideas. In fact, Lampert and colleagues (2013) noted that TCs had questions about exactly how to elicit and respond to student thinking (e.g., how many student ideas to elicit during a discussion). This finding further supports the conclusion that TCs require support to develop skill in enacting responsive teaching practices.

It is the responsibility of teacher education programs to promote and provide opportunities for TCs to develop skill with teaching more responsively. As evidenced above, this work is difficult for experienced teachers, which indicates that TCs, who have very little classroom experience, need support from TEs to develop and enact responsive teaching 
practices. Thus, TEs who provide opportunities for TCs to approximate this work help them to gain valuable experience while under the guidance of a TE. In the next section, I discuss literature related to how TEs can use approximations of practice to support TCs' development.

\section{Approximating Practice}

Following the decomposition of practice is the third and final aspect of the teacher education framework - approximating practice — which allows for TCs to engage in simplified practice as they focus on, enact, and develop particular aspects of teaching (Grossman, 2011; Grossman, Compton, et al., 2009). The idea of approximating practice relies on participating in authentic activity to allow novices to engage more fully in a community of practice.

Approximations of practice vary in degrees of authenticity (Grossman, Compton, et al., 2009). In mathematics teaching, a less authentic approximation might ask TCs to watch a short video clip of students and then craft a response to a student question or comment. A more authentic approximation would be student teaching, which requires TCs to engage in the interactive work of teaching in real time, but still with support from a professional teacher.

The pedagogies that TEs use to approximate practice have come to be known as pedagogies of practice (Boerst et al., 2011; Grossman \& McDonald, 2008; Kazemi et al., 2015). This set of pedagogies may be divided into two subsets: pedagogies of investigation and pedagogies of enactment (Grossman \& McDonald, 2008). Pedagogies of investigation call for TCs to engage in systematic investigation and analysis of learning and teaching, which might occur through observation and/or videos of teaching, examination of student work, or reflection (Ball \& Cohen, 1999; Grossman \& McDonald, 2008). For example, TEs have used videotaped classroom interactions to orient TCs to student thinking and to develop their skill in noticing students' ideas (Richards \& Robertson, 2016; Stahnke et al., 2016). 
The second type of approximation of practice — pedagogies of enactment—are less prevalent in teacher education (Grossman \& McDonald, 2008). Pedagogies of enactment are designed to support TCs in actually doing the work of teaching in the context of university methods courses and may take a variety of forms: lesson planning, student interview assignments, or teaching a lesson (Ball \& Forzani, 2009; Grossman \& McDonald, 2008; Kazemi et al., 2015; Lampert, 2010). Traditionally, lesson planning assignments tend to be the most common pedagogy of enactment in teacher education. A focus on the interactive work of teaching, however, tends to be left to clinical experiences, which are not formatively productive (Wideen et al., 1998).

Incorporating pedagogies of enactment that involve the interactive work of teaching in methods classes requires the TE to take on a different set of responsibilities than they might in a more traditional methods course. For example, when using a pedagogy of enactment, the TE is responsible for helping TCs develop their knowledge of important mathematics and of how students make sense of the mathematics (Kazemi et al., 2009). Scaffolding is an important part of this process, but the scaffolds put in place by the TE depend upon the teaching practice being developed. For example, in an elementary mathematics methods course focused on eliciting and interpreting students' mathematical thinking, TEs provided TCs with a group of pre-designed tasks and prompts to use during interviews with elementary students (Sleep \& Boerst, 2012).

It is important for TCs to both investigate and enact teaching practice. McDonald and colleagues (2013) developed pedagogies to aid TEs as they engage TCs in investigating and enacting core practices to support their learning (see Figure 1). Although core practices are regularly carried out by teachers, they remain abstract to TCs unless they are situated in authentic work. For TCs to truly learn to enact these practices, they must be embedded in activities which 
can be enacted. These activities have come to be known as instructional activities (IAs) and they aid TCs in their development of teaching practice (Lampert \& Graziani, 2009; McDonald et al., 2013). As TCs learn to enact core practices, it is crucial that they use the responsive teaching moves (practical tools) at their disposal flexibly, rather than in a memorized, rote fashion; they must attend to the purpose of an IA or a teaching move and be able to identify instances in which it affords opportunities to work toward the goal of the lesson.

The use of IAs were first investigated in the context of teacher education for teachers of Italian as a foreign language (Lampert \& Graziani, 2009). The TEs in this program taught TCs to use content-specific routines (IAs), which specified how the teacher, students, and mathematical content would interact in the context of authentic problems (Lampert \& Graziani, 2009). Extending this work to mathematics teacher education, IAs serve to explicitly define teaching moves that are required to carry out tasks that are "cognitively demanding" for students, provide a structure for teacher-student interaction using those teaching moves, and engage TCs in the teaching and learning of core practices (Kazemi et al., 2009, p. 3). The use of IAs also enables the TE to better predict difficulties that may arise for the TC during enactment (Lampert et al., 2013). 


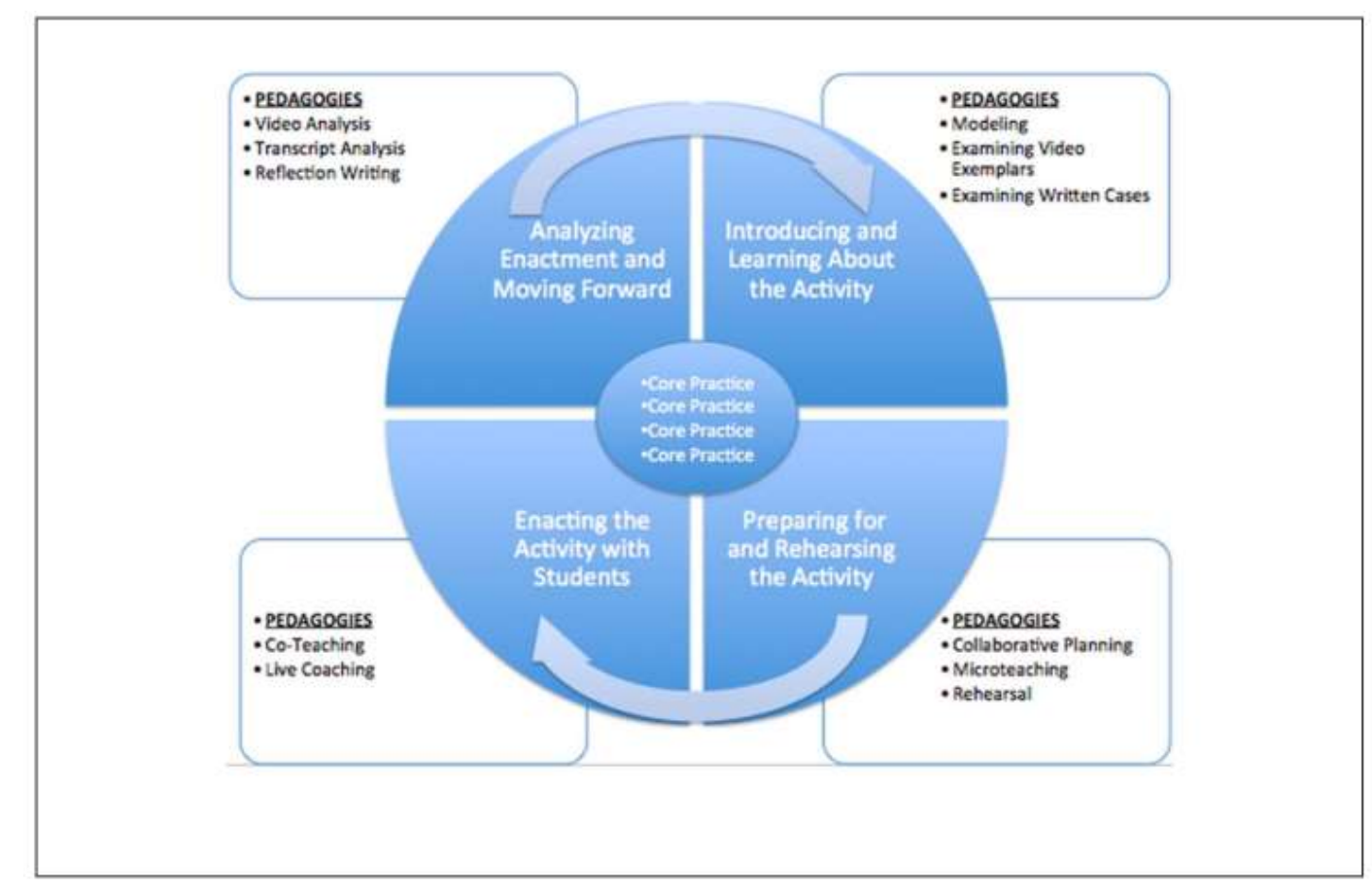

Figure 1. Cycle for collectively learning to engage in an authentic and ambitious instructional activity (McDonald et al., 2013)

This cycle, which I refer to as a cycle of investigation and enactment, centered on an IA, provides a way to support TCs as they develop flexibility in the hows and whys of teaching. As an introduction to the cycle, TCs first participate in an IA as students, while the TE leads the activity. Then, TCs break down the IA to analyze its parts and note the particular behaviors (including teaching moves) carried out by the TE. This analysis would serve to help TCs understand why certain moves were enacted at certain times.

The second part of the cycle calls for TCs to prepare to enact the IA as the teacher. This requires the TC to anticipate possible student responses (correct and incorrect) and to plan specific questions and teaching moves to enact. Then, TCs enact the IA publicly, in a methods classroom or public school classroom, which provides TCs with opportunities to understand how to enact teaching moves. (I will refer to TCs' IA enactments during methods as rehearsals.) The 
setting of the enactment affects its authenticity and may afford different learning opportunities for the TC. In a methods classroom, the students are other TCs who presumably have familiarity with the content being taught. In a school classroom, the students would be children who may or may not be familiar with the content. As a result, one might expect that they would ask different kinds of questions or make different kinds of comments than TCs in a methods course.

In either case, it is important to document the IA to allow the TC an opportunity to reflect upon the enactment. This documentation usually occurs as a video, but might also involve collecting and analyzing student work (McDonald et al., 2013). Finally, TCs come back together to analyze their enactments with the guidance of the TE. During this analysis, records of the TCs' practice (e.g., video recording, samples of student work) and student responses are used to examine how the newly learned teaching practices and the mathematical content worked together during the activity (Kazemi et al., 2015). This final step in the cycle provides an opportunity for TCs to reinforce and refine what they have learned about why particular teaching moves were (or could have been) enacted at particular times, as well as how those moves were (or could have been) enacted responsively.

The core practice being addressed affects the choice of the IA. To help learn the core practice of leading a mathematical discussion, for example, TEs engaged elementary mathematics TCs in IAs that involved solving, presenting, and discussing solutions to three-digit addition tasks (Tyminski, Zambak, Drake, \& Land, 2014). A sorting task, which asks students to sort card with examples and non-examples of a mathematical term, has been used with secondary mathematics TCs to help them work toward the mathematical purpose of refining a definition by building on students' ideas through whole-class discussion (Baldinger et al., 2016). To help learn the core practice of eliciting and responding to student ideas while teaching toward a 
mathematical goal, TEs engaged elementary mathematics TCs in a variety of IAs which included: (a) counting (e.g., by 10s, by 19s, by 3/4; Kazemi et al., 2009, p. 4), (b) strategy sharing, in which the TC poses a computational problem and elicits multiple strategies for solving the problem, (c) posing a sequence of related computational problems, and (d) solving word problems (Kazemi et al., 2009). While the studies described in this paragraph mention both elementary and secondary mathematics TCs, the research around IAs has primarily focused on elementary TCs. Thus, there is a need for more work to be done with a focus on preparing secondary mathematics teachers.

Coached rehearsals. The cycle of investigation and enactment requires TCs to rehearse IAs with their peers in methods courses and with students, both of which serve as versions of simulations of teaching. Simulated practice has played a role in training professionals, particularly in the medical field, for several years (Dotger, 2015; Haigh, 2007). The recent conception of teaching as a practice has encouraged TEs and researchers to implement similar experiences for TCs, often called rehearsals (Kazemi et al., 2009; Lampert et al., 2013; Lampert et al., 2015). Rehearsals are more than microteachings or "run-throughs" of lessons; they are meant to be close approximations of practice which allow TCs to experience authentic classroom discourse in a bounded environment that is sheltered from many of the distractions that occur in a public school classroom (Dotger, 2015; Lampert et al., 2013). In other words, rehearsals provide a means for TEs to reduce the complexity of teaching in order to provide TCs with opportunities to focus on particularly salient aspects of the work (Dotger, 2015; Grossman \& McDonald, 2008). Ultimately, the purpose of rehearsals is to "shift teacher preparation from what the $[\mathrm{TC}]$ knows to what she or he can and will actually do" (Dotger, 2015, p. 7). For example, when Lampert and colleagues (2013) investigated the core practice of eliciting and 
responding to student thinking, rehearsals were carefully designed so that TCs could learn about questions to ask in order to elicit student thinking. In addition, TCs were provided with opportunities to consider how many student responses to elicit and how to respond to students' ideas.

Instructional coaching is an important part of a rehearsal. Schon (1987) argued for TCs' need for opportunities to develop their skill in relational practices in low-risk settings with focused coaching by someone who is already a professional teacher (as cited in Grossman, Compton, et al., 2009). Joyce and Showers (1981) note the importance of coaching as it relates to TCs' transfer of knowledge across settings (i.e., from methods classroom to school classroom). In the role of "knowledgeable other" in the methods classroom, the TE typically acts as the instructional coach. In practice, this means the TE may pause the rehearsal to highlight a particular teacher-student or student-student interaction or teaching move that is worthy of discussion (Grossman, Compton, et al., 2009). The TC is also permitted to pause the rehearsal to ask questions of the coach. For example, a TC might be unprepared to discuss an idea that has been offered by a student in the class and may ask the TE for the best approach for continuing the discussion. In either case, the immediate feedback offered through coaching is an important distinguishing feature from pedagogies involving microteaching. With microteaching, feedback is reserved for the end of a TC's presentation and may be given privately, preventing other TCs from benefitting from the work of their peer. The publicity of the feedback that results from coaching allows for a more efficient development process for TCs. All TCs in a methods course can benefit from just one comment from the coach, whereas comments related to microteaching would have to be repeated to students, one at a time. 
This is not to suggest, however, that instructional rehearsals and coaching provide an immediately perfect organization for methods courses. TEs must plan for rehearsals carefully by choosing IAs that will highlight the desired responsive teaching practices, ordering the IAs in a logical sequence, and determining which (if not all) TCs will rehearse a particular IA and in what order. Coaching is also complex work, requiring the TE to decide how to interrupt (e.g., by asking a question, suggesting a teaching move, highlighting a successful move) and how often to interrupt (Baldinger et al., 2016). The TE must also balance feedback about more routine aspects of teaching (e.g., where to stand, how loudly to speak) with feedback about more complex aspects (e.g., how to orient students to one another's ideas; Lampert et al., 2013). In addition, the methods instructor must consciously avoid being reluctant to give critical feedback and be mindful of the possibility that the coaching may reflect their own idiosyncrasies, potentially making it unbalanced (Lampert, 2010).

\section{Motivating This Study}

Responsive teaching has been shown to be beneficial to students' conceptual understanding and achievement, but teachers (and TCs) must learn about both practices and moves that promote responsive teaching, as it is not easily learned (Ball \& Cohen, 1999). Richards \& Robertson (2016) acknowledge that "much work remains to be done in understanding how teachers take up, apply, and/or adapt the various 'ways in' to responsive teaching" (p. 50). Consequently, much work also remains to be done to understand how TEs are taking up the work of developing responsive teachers.

Teacher education must deliberately focus on the development of skill with core practices of teaching, particularly the practices related to how a teacher elicits and responds to student reasoning and makes those ideas central in a classroom discussion toward established goals 
(NCTM, 2014; Sleep, 2012). This study is framed by a particular view of what it means for a TC to develop skill with responsive teaching practices. This development involves more than merely enacting what researchers have classified as responsive teaching moves. Focusing strictly on whether TCs enact the moves would promote TCs' development of responsive teaching skills at a smaller grain size (i.e., the how of responsive teaching practices) without providing opportunities for them to examine responsive teaching at a larger grain size (i.e., the why of responsive teaching practices; Boerst et al., 2011). From this viewpoint, there are a several aspects of responsive teaching that must be considered when examining TC development.

TCs may develop a variety of responsive teaching skills simultaneously: purposeful enactment of moves, timely enactment of moves (aimed at maximizing their responsive potential), and the execution of responsive teaching moves in responsive ways. An example of this might be when a TC asks a student to revoice an idea that was imprecisely or unclearly stated by another student. In such an instance, the purpose of the move might be to more clearly articulate a student idea to facilitate a better understanding of the idea by the class. By enacting the move immediately following an unclear statement of a student idea, the TC stands to maximize the responsive potential of the revoicing move. Thus, as I consider TC development throughout this dissertation, I examine not only the number of enactments of responsive teaching moves, but I also look for evidence of why TCs chose to enact particular moves in particular moments, as well as the outcomes of those moves, to determine the extent to which they were executed responsively.

In addition to helping TCs develop those skills in the "controlled setting" of a methods classroom, TCs must also make progress in doing this work skillfully in the "authentic setting" of public school classrooms (McDonald et al., 2013). An understanding of which skills TCs 
transfer to the school setting will provide TEs with much-needed information about how to change the design of their courses to positively influence TCs' teaching practice. It is also important to note that, in the field of mathematics education, much of the work in responsive teaching has involved elementary TCs or practicing teachers; little has been done with secondary mathematics TCs.

Pilot study. My attention to this work is further motivated by a pilot study I conducted during the spring semester of 2016. The study focused on the ways TCs taught responsively during coached rehearsals and how the coaching affected TC responsiveness. That work yielded some early observations and findings about supporting TCs' responsive teaching practice through the use of coached rehearsals.

Throughout the semester, teacher revoicing was the most commonly enacted responsive teaching move, perhaps because it is relatively easy to enact. It is also interesting to note that the teacher revoice move was often related to recording-TCs enacted this move when they wanted to ensure they correctly represented a student's idea on the board. Making connections among students' ideas was the least common responsive teaching move, supporting Dyer and Sherin's (2015) conclusion that experience is required to identify and articulate connections among ideas that have just been contributed.

Although responsiveness was not necessarily a focus of the instructional coaching that took place during rehearsals, the pilot study revealed that the majority of the coaching interjections, whether they were directives or compliments toward the rehearsing TCs, were focused on responsiveness. When the rehearsing TCs asked a question of the coach almost half of the instances were also focused on responsive teaching, which suggests that TCs were 
attending to responsive teaching, even though it was not a deliberate focus of the methods course.

Research Questions. While all of these findings provide some valuable insight into TCs' development in responsive teaching, what still remains unclear is the extent to which TCs incorporated those responsive teaching skills in school classrooms. For this dissertation study, I use a working definition for responsive teaching in teacher education, drawing on the literature and the results of my pilot study, to examine how TCs develop skill in responsive teaching. I examined these phenomena in the context of a secondary mathematics methods class and school placements with an eye toward the responsive teaching moves enacted by the TCs and the substance of the coach's feedback, to identify how the feedback supported responsive teaching. I also examined how those moves evolved in a different context - the classroom. In particular, the following questions guided my study:

1. How do TCs develop skill in teaching mathematics responsively within a methods course in which cycles of investigation and enactment are a central pedagogy?

2. How does coaching in methods affect TCs' responsive teaching?

3. To what extent do TCs teach responsively in school settings?

Pursuing these questions will contribute both to a sense of how TCs develop skill with responsive teaching, as well as the teacher education designs that support that development. In Chapter 3, I will discuss the methods I used to pursue these research questions. 


\section{CHAPTER THREE}

\section{Methods}

I drew upon previous research design recommendations from the literature, as well as the design of the methods course that was the focus of my pilot study, to inform the design of the methods class that was the primary setting for my study. This shares some aspects of designbased research (DBR) as it focuses on the interplay between design and theory, uses previous design iterations to inform future designs, and focuses on the context in which learning occurs (Barab \& Squire, 2004; Design-Based Research Collective [DBRC], 2003). Unlike DBR, however, my study does not include iterations in which emerging findings are applied to the ongoing course and research designs.

In helping TCs develop skill in both how and when to enact responsive teaching practices in the methods class, the eventual goal was to enable them to enact those same practices successfully and judiciously in school classrooms. My study considered TCs' teaching in both contexts as I examined how TCs implemented practical tools (e.g., responsive teaching moves) from the methods course in their school placements. In the sections that follow, I first describe the design of the methods course, including important features of the design. I then discuss my research of the implementation of the course design.

\section{Course Design Features}

Several aspects of the methods course drew directly upon successful designs found in the literature. For example, in previous research involving cycles of investigation and enactment, IAs, and coached rehearsals, researchers have found that using these pedagogies in teacher education creates a space in which TCs and TEs can work together to study the practice of teaching (Kazemi et al., 2009). Kazemi and colleagues (2015) found that coached rehearsals 
require a supportive classroom culture in which TCs' practice is made public and that TEs (acting as coaches) must support TCs in taking risks by providing strategic feedback on TCs' teaching. In my study, I used these pedagogies to create a collaborative space that had responsive teaching as its focus. As the coach, I aimed to provide judicious feedback to TCs which supported them as responsive teachers.

However, in response to gaps in the literature or practical considerations specific to my study, some elements of previous teacher education designs were adapted. While one intent of the adaptations was certainly to improve the design for future iterations, some of the adaptations were necessary to accommodate the context of my methods course. In particular, I made the following adjustments: adapted the cycle of investigation and enactment put forward by McDonald and colleagues (2013; see Figure 1), focused specifically on responsive teaching practices (as opposed to a set of more general core practices), and designed the course for secondary mathematics TCs in particular, which included choosing IAs that were appropriate for secondary students. Further descriptions of these changes follow in the sections below, including a rationale for each.

Responsive teaching practices. My methods course design involved a deliberate focus on responsive teaching practices (i.e., core practices that have responsive teaching as their focus), which were discussed in detail in Chapter 2. Here, I aim to motivate the need for a connection between responsive teaching practices and pedagogies of practice.

Two separate bodies of literature exist which have focused on either core practices (through the use of pedagogies of practice) or responsive teaching, but have not considered how they may be linked. Both offer important ideas for TC development. While some of the core practices discussed in math education literature would fall under my heading of "responsive 
teaching practices," previous work in this area has not deliberately focused on responsiveness. Because my course design involved the articulation of and semester-long focus on responsive teaching practices, TCs were given opportunities to develop skills such as eliciting and responding to student reasoning and orienting students to one another's ideas. As the instructional coach, I used responsive teaching practices as a lens through which to view TCs' rehearsals and aimed to make judicious comments to aid TCs' development of enacting such practices. Responsive teaching moves that seemed to be particularly difficult for TCs to enact in responsive ways became a focus of our whole-class discussions and/or the focus of targeted comments, either as coaching interjections during rehearsals or in response to TCs' video annotations.

Adaptation of cycle of investigation and enactment. Kazemi and colleagues (2015) note that rehearsals are shaped by how closely they occur to enactments with children. In my pilot study, TCs were not required to enact any of the IAs with public school children, making it impossible to know whether they were applying what they were learning in the methods class when they were in their school placements. My study required TCs to enact one IA with students in schools as a means of encouraging TCs to consider how the work being done in methods could be applied in school classrooms. This also made it possible for me to study how TCs implemented pedagogical tools (e.g., responsive teaching moves) from the methods course in their school placements. (See research of methods course implementation section that follows.)

However, enactment with children was not part of each three-week IA cycle. I adapted the cycle of investigation and enactment put forward by McDonald and colleagues (2013), mainly as a result of practical considerations related to the context of the methods course. (See Figure 2.) The course met on a university campus, three times per week (as opposed to being 
situated in a secondary school, which would have provided frequent access to school teachers and students). Three of the TCs involved in the study were required to complete a 75-hour school placement for a different education course. (The fourth TC would complete the 75-hour school placement in an upcoming semester.) However, they often do not receive their placement assignments until the fifth or sixth week of the semester. Since the school placement is attached to another course, any assignments TCs are asked to complete in schools primarily fulfill requirements for that course. Therefore, TCs had minimal time to enact IAs in schools. I asked TCs to enact only one IA during their placement, with the time and topic agreed upon by the TC and the host teacher. TCs video recorded their school enactments, uploaded them into Vosaic Connect ${ }^{2}$, and then used the software to reflect upon and annotate their enactment of the IA.

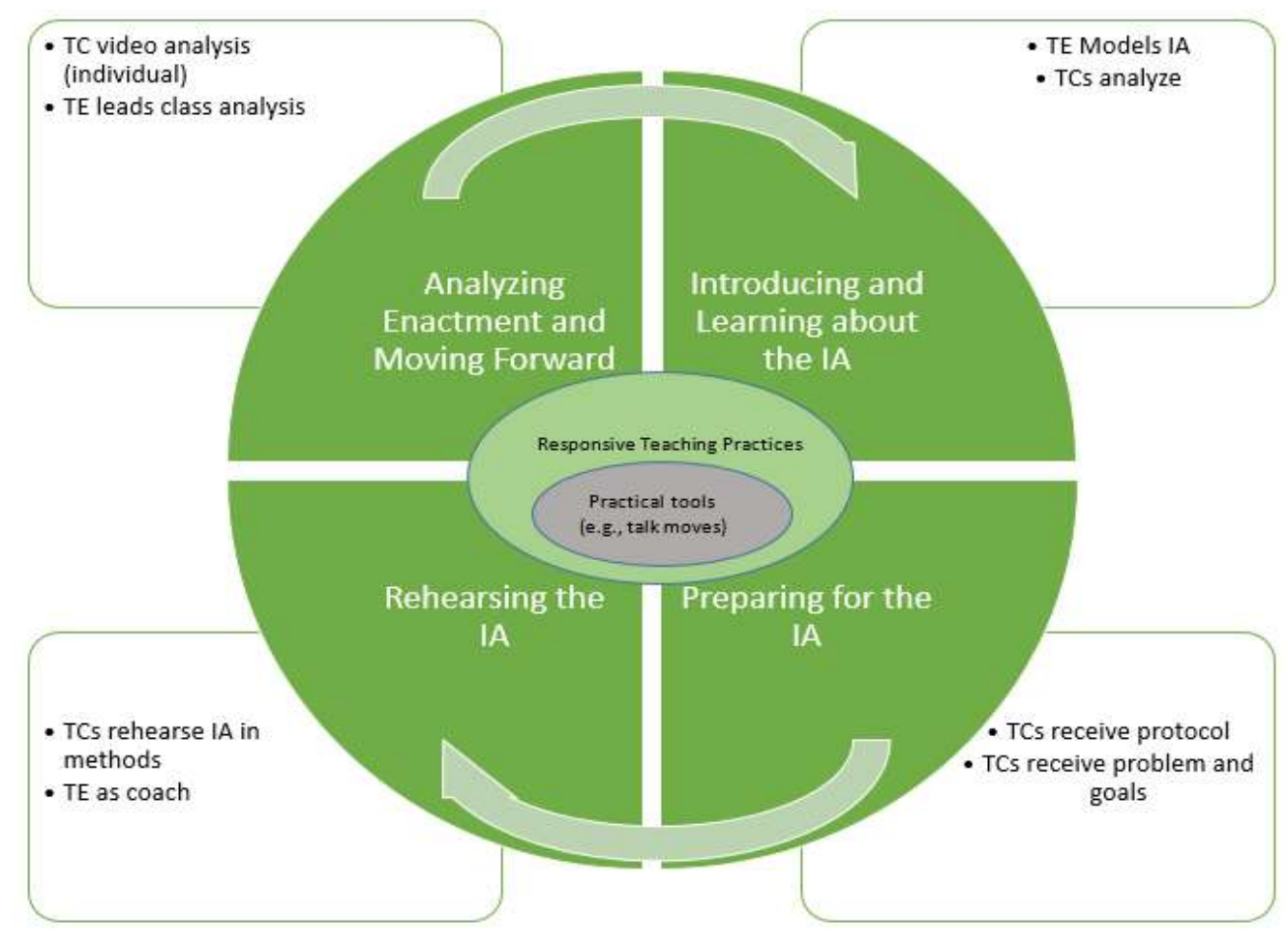

Figure 2. Cycle of Investigation and Enactment in Mathematics Methods Course

\footnotetext{
${ }^{2}$ Vosaic Connect is a video analysis software. Once a video has been uploaded, users can identify and comment on notable moments. Each TC and I had access to Vosaic Connect throughout the methods course.
} 
Implementation of cycle of investigation and enactment. During the second week of the course, TCs enacted a brief Teaching Demonstration (Teaching Demo) in the methods course. Each TC was given a problem (without identified goals) to go over with the class, using a method of their choice, to provide baseline data for their teaching. During the third week, TCs began the first of four IA cycles. In keeping with the cycle of investigation and enactment, each IA was the focus of instruction for three weeks (McDonald et al., 2013). Four different IAs were chosen based on their usefulness in secondary mathematics classrooms. Activities during the first week of each IA cycle corresponded with the first part of the cycle of investigation and enactment: introducing and learning about the activity. I allowed the TCs to experience the IA as learners and then led a discussion during which the TCs analyzed the mathematics and the practices and moves that were involved in the IA. For instance, after TCs participated in the Going Over a Problem IA, we noted the importance of giving students time to think about the problem and to solve it on their own. During this class session we also discussed how to productively elicit student strategies during the whole-class discussion (i.e., by watching students as they work and then calling on someone to respond, rather than broadly asking, "Who would like to share how they solved this?"). At the end of the first week, TCs were given a protocol that outlined the structure of the IA, a set of recommended moves, and an assigned problem with corresponding mathematical goals, all of which they used to prepare to enact the IA. This corresponded to the second part of the cycle of investigation and enactment: preparing for the activity.

During the second week of the IA cycle, TCs rehearsed the IA in the methods class as I fulfilled the role of instructional coach. I recorded the rehearsals and uploaded the videos to Vosaic Connect. TCs were asked to annotate and reflect upon their rehearsals in response to a set 
of reflection prompts. During the third week, I led the TCs in a whole-class reflection of the previous week's enactments by reviewing particularly salient instructional moments as they related to the core practice(s) being emphasized. This was consistent with the final phase of the cycle of investigation and enactment: analyzing enactment and moving forward. After the completion of one three-week IA cycle, TCs were introduced to a new IA the following week. (The IA cycle is summarized in Figure 2.)

Once the first IA cycle was complete, TCs were asked to enact the first IA (Going Over a Problem) at least once in their school placements. The date of enactment and topic of the IA was determined by the TC and the mentor teacher, although I did assist the TCs in ensuring that the problems they selected would be appropriate for the IA. TCs were asked to record their school enactment, upload it to Vosaic Connect, and then annotate and reflect upon it, using the same annotation and reflection prompts that were used for the rehearsals.

While rehearsals were central to the structure of the course, it is important to note that this was not the only course focus. To meet accreditation requirements, TCs in the methods course were required to complete an instructional unit and a math history project. There were also other assignments incorporated into the course to help students prepare for their action research projects, which they are required to complete during their student teaching semester. (See Appendix A for a weekly course schedule.)

Secondary math education. My methods course design is intended for secondary mathematics TCs' development, a population which is underrepresented in the literature at present, particularly as it relates to the enactment of responsive teaching practices. Several studies have been conducted with elementary mathematics TCs and their enactment of teaching, but less work has been done with secondary mathematics TCs (Boerst et al., 2011; Kazemi et al., 
2009; Sleep \& Boerst, 2012; Tyminski et al., 2014). Of the few studies that have been conducted with secondary mathematics TCs, the focus has not been on responsive teaching practices. For example, Baldinger and colleagues (2016) focused on TCs' use of a particular IA (card sort). Other work, while focused more on responsive teaching, was done with practicing secondary mathematics teachers, rather than TCs (Dyer \& Sherin, 2015; Van Zoest et al., 2016).

Drawing on studies that have been conducted with elementary TCs provided a basis for the design of my methods course, which involved creating situations through which TCs could participate in particular forms of learning about core practices of the work of teaching through the use of rehearsals and IAs (Cobb, Confrey, diSessa, Lehrer, \& Schauble; 2003). Working with secondary TCs necessitated the use of a different set of IAs than were used with elementary TCs. For example, rather than asking TCs to enact an IA focused on counting (Kazemi et al., 2009), the IAs chosen for this design focused on topics like comparing graphical representations of polynomial functions. In the next section, I provide a detailed overview of each of the IAs that were chosen for the methods course.

Overview of IAs. Four IAs (not including the initial Teaching Demonstrations) were chosen for use in the methods class, based on their usefulness in highlighting responsive teaching practices and their practicality in secondary mathematics classrooms (considering both content and time constraints). Together they provide opportunities for TCs to work with a variety of mathematical topics and responsive teaching moves. For example, the first two IAs provided students with actual problems to solve, which allowed the TCs to elicit and respond to students' strategies. The last two IAs did not involve finding solutions to problems, but, instead asked students to make comparisons and justify their thinking. During these IAs, rather than eliciting 
solution strategies, TCs elicited observations about different representations of a mathematical term (e.g., graphs of linear equations, systems of equations).

By enacting IAs publicly through rehearsals, TCs learned about important teaching practices not only while they led the discussion, but also as they participated in and observed IAs as enacted by their classmates. The IA protocols and evaluation rubric were written with the aim of helping TCs focus on the responsive teaching practices of eliciting and responding to student thinking and orienting students to one another's ideas. By allowing each TC the opportunity to enact four different IAs, the intent was for them to develop skills and teaching moves that would carry over into their clinical and student teaching experiences.

Going Over a Problem (GOP). The first IA enacted by TCs was Going Over a Problem, in which each TC was given a problem to go over with the class, along with mathematical goals for the discussion (Campbell, 2016). As teachers of mathematics, TCs will inevitably be going over problems with their students on a daily basis. As an alternative to the teacher displaying a single solution strategy for students to passively observe or examine, this IA uses "typical" problems (i.e., the problems are not word problems or are no at a particularly high level of difficulty) to engage students in discussion around different strategies that may be used to solve the problem. For TCs, this IA allows opportunities for developing skill in eliciting and responding to student reasoning and orienting students to one another's ideas. TCs worked with this IA during weeks 3-5 and were also asked to enact this IA in their school placements sometime during the semester.

Contemplate then calculate (CthenC). The second IA of the semester was Contemplate then Calculate (Kelemanik, Lucenta, \& Creighton, 2016). The aim of this IA is to help TCs encourage students to consider how to leverage the structure of a computation problem, rather 
than to immediately rush to solve the problem with a standard algorithm. (As part of the Common Core State Standards for Mathematics [CCSSM], eight Standards for Mathematical Practice were implemented to help students develop "important "processes and proficiencies" in mathematics [CCSSM, 2017]. The Contemplate then Calculate IA speaks to the seventh Standard for Mathematical Practice - "look for and make use of structure.") This IA is wellsuited for any secondary mathematics classroom in that the chosen problem can come from any mathematical domain (e.g., algebra, geometry, statistics, etc.) and be made appropriate for any secondary grade level. Although not required for the methods class, TCs could have reasonably enacted this IA in their school placements as a warm-up activity in the first 10-15 minutes of class. TCs worked with this IA during weeks 6-8 of the semester.

Card Sort. The third IA cycle centered on the Card Sort IA, which focuses on defining a mathematical idea (Baldinger, Selling, \& Campbell, 2015). Precise definitions are crucial in mathematics, but students may have difficulty in understanding why precision is required or how to discuss mathematical ideas using precise language. As a result, the Card Sort IA requires TCs to lead a different type of discussion. Rather than discussing solution strategies to a mathematical task (as in the first two IAs), TCs must guide the discussion as student clarify and refine a mathematical definition.

During the IA, students are asked to sort a set of cards as examples or non-examples of a mathematical idea (e.g., linear equations). Some of the cards in the set are intended to be difficult to sort in order to generate discussion about why a single card might seem to be classified as both an example and a non-example. Once several (but not all) of the cards have been discussed, students are asked to craft a definition of the mathematical idea which incorporates the characteristics of the cards that were sorted as examples. After eliciting students' ideas, the 
formal definition is given and, as a closure, students are asked to sort one or two more cards to ensure their understanding of the definition. TCs worked with this IA during weeks 9-11 of the semester.

Which one doesn't belong (WODB). The fourth and final IA was Which One Doesn't Belong (Campbell, 2016). It is similar to the Card Sort IA in that it is less concerned with solution strategies to a mathematical task. Instead, it is more concerned with identifying and articulating differences between seemingly similar mathematical objects (e.g., equations of linear functions). Leading a classroom discussion around this IA is also somewhat similar to leading a discussion around a Card Sort, since the focus is on hearing and organizing students' arguments and justifications for why a tile does not belong. However, unlike the Card Sort, the goal of WODB is for students to examine the properties of the mathematical objects themselves without necessarily working toward a rigorous mathematical definition. I chose WODB to be the final IA because each TC was given several goals for their rehearsal of this IA and thus, had multiple ideas upon which to focus the discussion. It was my hope that WODB would be more manageable for TCs after rehearsing the Card Sort IA, whose discussion has a similar feel, but only a single goal.

During WODB, students are shown four tiles displaying mathematical objects. They are asked to choose one and justify why it does not belong in the same category as the other three. The tiles are designed so that any one of the four might be the one that does not belong. Therefore, if students at first only offer rationales for why one or two of the tiles do not belong, the TC then directs students to consider each remaining tile and why it might be the one that does not belong. The IA closes with students articulating specific characteristics that should be considered when working with that particular type of mathematical object. (Appendices B, C, D, 
and E, contain planning materials for each IA, respectively. TCs worked with this IA during weeks 12-14 of the semester. Appendix F contains the problems/prompts and goals for the Teaching Demonstrations, rehearsals of each IA in methods, and TCs' school enactments.)

Video and video annotation. TCs' rehearsals of the IAs in methods were video recorded, which allowed each TC to analyze and reflect upon their practice individually. The videos also contributed to the whole-class discussions that occurred during the third week of each IA cycle as I (TE) chose salient video excerpts to watch and discuss as a class. TCs were asked to watch and reflect upon their own rehearsals, using Vosaic Connect to identify salient moments in their teaching. Baldinger and colleagues (2016) showed that structured reflections aid TCs in conducting a more "targeted analysis" than asking more general questions like, "what might you have done differently" (p. 26). In response to this result, I provided TCs with a series of prompts aimed at helping them focus their reflections on their own rehearsals.

TCs were first asked to identify four types of moments during their rehearsals:

(1) moments when they focused on student reasoning, (2) moments when they were making progress toward their mathematical goal(s) for the IA, (3) moments they found particularly challenging, and (4) moments when they either felt supported by or felt they need more support from the coach. After identifying these types of moments, TCs were asked to reflect on each moment individually to explain why it was chosen. See Appendix G for video annotation instructions and prompts. TCs' participation in this form of reflection aimed to further their development as responsive teachers by encouraging them to consider more objectively the intent, enactment, and results of specific teaching moves. Their reflections allowed them an opportunity to consider more responsive alternatives to the moves that may not have been as responsive as they could have been. 


\section{Research on the Implementation of the Methods Course Design}

Systematically studying an implementation of my methods course design helps in further understanding the nature of TC development, which has implications for design revisions in future iterations of the course (Barab \& Squire, 2004; Campbell, 2014; Cobb et al., 2003; DBRC, 2003). For example, my design of the study requiring that TCs enact one IA (Going Over a Problem) in schools made it possible for me to study how TCs implemented pedagogical tools (e.g., responsive teaching moves) from the methods course in their school placements. This requirement will also inform future iterations of the methods course by allowing me to consider whether substantial conclusions can be drawn based on data that is collected in a single semester with only one IA enactment occurring in schools.

In the sections that follow, I provide detailed information about my research procedures and methods of analysis, beginning with some background information about the participants and context of my study.

\section{Participants and Context}

The study of the implementation of the methods course design took place in a secondary mathematics methods course at a small university in north central West Virginia. All contentspecific methods courses are taught in their respective content departments. Thus, this 16-week methods course is housed in the mathematics department and is offered annually; I am the only instructor. I have nine years of experience as a high school mathematics teacher and, at the time of the study, I had two years of experience as a mathematics teacher educator. I taught this threecredit methods course for the third time during the fall 2017 semester. We met three times per week; each session was 50 minutes in length. 
Since this is the only methods course that secondary mathematics education majors take, various facets of mathematics teaching must be discussed in a short period of time. In my experience, the TCs in this course generally have little to no experience in finding or reading mathematics education research, thoughtfully selecting and implementing mathematics-specific instructional tools and technologies, or attending to mathematics-specific considerations for lesson planning. These skills, in addition to the attention given to interactive teaching practices, must all be addressed in a three-hour methods course.

The participants in the study were four undergraduate secondary mathematics education TCs in their senior year of coursework: Bridget, Charity, Grace, and Julian (all pseudonyms). These TCs comprised the entirety of my secondary mathematics methods course. All of the TCs were traditional students (under the age of 25) and were originally from West Virginia. Grace was pursuing a degree in secondary mathematics education, which leads to a certification to teach mathematics in grades 5-adult; this was her only required mathematics methods course. Bridget, Charity, and Julian were pursuing elementary education degrees with specializations to teach middle school mathematics (grades 5-9). In addition to the secondary methods course, they were also required to complete an elementary mathematics methods course, which is concerned with the teaching of mathematics in grades K-6. All three students had successfully completed elementary mathematics methods before enrolling in the secondary mathematics methods.

Bridget, Charity, and Grace were concurrently enrolled in an education course that requires a 75-hour clinical experience during the semester, which allows TCs access to practicing teachers and public school students. All three of these students were placed in middle school mathematics classrooms - Charity in 6th grade and Bridget and Grace in 8th grade classrooms. Julian, although not concurrently enrolled in this education course, was still able to gain access 
to practicing teachers and public school students via a teacher from a previous school placement. Therefore, Julian worked with a 6th grade mathematics class to complete assignments for the methods course, which did not require a 75-hour commitment. As a result, Julian did not have the same opportunities as Bridget, Charity, and Grace to develop relationships with students. Still, he was able to participate in all aspects of this study.

My roles as researcher and instructor. As both researcher and instructor, I held several sometimes competing roles throughout the semester. As the researcher, my responsibilities involved collecting, organizing, and analyzing the data. As the instructor, it was my responsibility to introduce the IAs during the first week of each IA cycle, to act as the instructional coach during rehearsals - to pause the IAs to ask questions or provide feedback as needed - and to lead the whole-class reflection during the third week of each IA cycle. To ensure the rigor of my study, I kept a reflective journal to catalog my thoughts after each class session that was involved in the study (Altheide \& Johnson, 1994; Lincoln \& Guba, 1985). Illustrations of the utility of the journal come later in the chapter.

Because the course had only four TCs, and one of them was leading the IA, only three TCs remained to be students for the IA. Further, there were not enough TCs to split the class into small groups, an important requirement of each of the four IAs. Therefore, in addition to being the coach, I also participated as a student, which enabled the class to be split into two pairs for each IA. (This was successful during most IAs. If a TC happened to be absent, I participated exclusively as a coach while the two "student" TCs participated in a single group for that particular IA.) As a student participant, I attempted to ensure that multiple solutions or strategies were brought forward; TCs needed opportunities to make decisions about which student ideas to foreground for the whole-class discussion and in what order those ideas would be discussed. 
Sometimes, this involved introducing common errors, while at other times it involved introducing correct, but unexpected strategies (e.g., simplifying $3 \frac{11 / 4}{x} 4 \frac{1}{3}$ using the distributive property).

\section{Data Collection}

I collected various types of data throughout each IA cycle, each of which made specific contributions to my three research questions. Table 2 provides an overview of the data sources and how they connected to each research question. In the sections that follow, I provide detailed descriptions of the data sources and data collection methods. 
Table 2

Research Questions, Data Sources, and Data Collection

\begin{tabular}{|c|c|c|}
\hline Research Question & Data Source(s) & Data Collection \\
\hline \multirow{6}{*}{$\begin{array}{l}\text { How do TCs develop skill in teaching } \\
\text { mathematics responsively within a methods } \\
\text { course in which cycles of investigation and } \\
\text { enactment are a central pedagogy? }\end{array}$} & Video - rehearsals & $\begin{array}{l}5 \text { recordings in methods per TC } \\
\text { ( } 1 \text { Teaching Demonstration; } 4 \text { IAs) }\end{array}$ \\
\hline & Video annotation/reflection assignment & $\begin{array}{l}4 \text { per TC (following each IA rehearsal) } \\
\text { [Appendix G: Prompts 1-3] }\end{array}$ \\
\hline & Planning materials & 4 per TC (one set of materials per IA) \\
\hline & Interviews (Pre, Mid, Post) & 3 interviews per TC \\
\hline & & [Appendix H: Questions 1-3] \\
\hline & Reflective Journal & Weekly entries (at least) \\
\hline \multirow[t]{6}{*}{$\begin{array}{l}\text { How does coaching in methods affect TCs' } \\
\text { responsive teaching? }\end{array}$} & Video - rehearsals & $\begin{array}{l}4 \text { recordings in methods per TC (one per } \\
\text { IA) }\end{array}$ \\
\hline & Video annotation/reflection assignment & 4 per TC (following each IA rehearsal) \\
\hline & & [Appendix G: Prompts 3-4] \\
\hline & Interviews (Mid, Post) & 2 interviews per TC \\
\hline & & [Appendix H: Questions 4-5] \\
\hline & Reflective Journal & Weekly entries (at least) \\
\hline \multirow{4}{*}{$\begin{array}{l}\text { To what extent do TCs teach responsively in } \\
\text { school settings? }\end{array}$} & Video - classroom & 1 per TC \\
\hline & Video annotation/reflection assignment & $\begin{array}{l}1 \text { per TC placed in classroom } \\
\text { [Appendix G: Prompts } 1-3 \text { ] }\end{array}$ \\
\hline & Planning materials & 1 set per TC \\
\hline & Interviews (Post) & $\begin{array}{l}1 \text { interview per TC placed in classroom } \\
\text { [Appendix H: Question 6] }\end{array}$ \\
\hline
\end{tabular}


Video. Several types of data were collected throughout the study. All class sessions dealing with rehearsals were video recorded to allow for an examination of TCs' general development in responsive teaching, as well as their use of responsive teaching moves in their responsive teaching. Table 3 displays the dates of video recordings, the order in which the TCs led each IA, and the length of each IA. The results of my pilot study indicated that only having video recordings from the methods class limited my understanding of TCs' development. In addition, others have found that TEs have little impact on teaching practices in the K-12 setting (Lampert, 2010). Consequently, TCs were asked to record the enactment of at least one IA in their school placement. This allowed me to see the extent to which TCs used the tools that had been introduced in the methods course in their school placements.

Table 3

Date, Order, and Length of IAs

\begin{tabular}{ccccc}
\hline IA & Date & Order & TC & Length of IA \\
\hline Teaching Demo & $8 / 21 / 17$ & 1 & Grace & $5: 10$ \\
& $8 / 21 / 17$ & 2 & Julian & $3: 25$ \\
& $8 / 21 / 17$ & 3 & Charity & $3: 08$ \\
& $8 / 21 / 17$ & 4 & Bridget & $7: 51$ \\
GOP & $9 / 6 / 17$ & 1 & Grace & $13: 41$ \\
& $9 / 6 / 17$ & 2 & Bridget & $17: 06$ \\
& $9 / 8 / 17$ & 3 & Julian & $15: 31$ \\
& $9 / 8 / 17$ & 4 & Charity & $20: 30$ \\
CthenC & $9 / 25 / 17$ & 1 & Charity & $15: 21$ \\
& $9 / 25 / 17$ & 2 & Julian & $19: 01$ \\
& $9 / 27 / 17$ & 3 & Grace & $19: 41$
\end{tabular}




\begin{tabular}{ccccc}
\hline IA & Date & Order & TC & Length of IA \\
\hline \multirow{3}{*}{ Card Sort } & $9 / 27 / 17$ & 4 & Bridget & $14: 03$ \\
& $10 / 16 / 17$ & 1 & Charity & $29: 41$ \\
& $10 / 18 / 17$ & 2 & Grace & $37: 31$ \\
& $10 / 20 / 17$ & 3 & Bridget & $25: 56$ \\
WODB & $10 / 23 / 17$ & 4 & Julian & $23: 41$ \\
& $11 / 6 / 17$ & 1 & Bridget & $22: 16$ \\
& $11 / 6 / 17$ & 2 & Grace & $23: 18^{\mathrm{a}}$ \\
& $11 / 8 / 17$ & 3 & Julian & $26: 05$ \\
& $11 / 8 / 17$ & 4 & Charity & $23: 26$ \\
& $11 / 8 / 17$ & -- & Charity & $24: 44$ \\
& $11 / 13 / 17$ & -- & Julian & $8: 51$ \\
& $11 / 13 / 17$ & -- & Grace & $19: 51$ \\
& $11 / 17 / 17$ & -- & Bridget & $10: 54^{\mathrm{b}}$ \\
\hline
\end{tabular}

${ }^{a}$ Video equipment malfunctioned - entire IA not recorded

${ }^{\mathrm{b}} \mathrm{IA}$ was recorded from start to finish, but there seem to be gaps during times when students were working independently and/or in groups.

Video annotation. As a means of hearing the TCs' voices through this research, written data was collected in the form of video analysis and reflections. Following each IA enactment, TCs were asked to watch their rehearsal videos in Vosaic Connect and provide annotations based on a series of prompts (see description of this design feature in the presentation of the design of the methods course above). This data provided insight into how TCs viewed their own practice and how their perceptions evolved over the course of the semester, which allowed me to consider TCs' developing skill in responsive teaching more deeply than if I had only viewed their rehearsals from my own perspective. For example, through TCs' video annotations, I was able to gain a better understanding of their purposes for enacting particular moves in particular 
moments. Their annotations also provided me with a glimpse of what they noticed about students and their own practice. These written reflections were connected to the identified moments and stored in Vosaic Connect. As the instructor, I read and responded to their reflections as necessary; my comments are also stored in Vosaic Connect.

Planning materials. In addition to video data, I also collected TCs' planning materials following each IA enactment. For each IA, TCs submitted a pre-planner, which was designed to help TCs carefully consider the mathematics behind their prompt and the mathematical goals of the IA. TCs also submitted a protocol for each IA, which was designed to help them think through the enactment of the IA, in part by writing notes for themselves about what questions to ask and what teaching moves to enact at particular moments. TCs' planning materials provided further insight into how TCs were thinking about responsive teaching prior to their IA enactments. The level of attention given to student thinking before each IA provided evidence related to TCs' development in that aspect of responsive teaching. The planning materials also provided insight related to the teaching moves TCs planned to enact. Although TCs might not enact every responsive teaching move as planned, an examination of their planning materials revealed some development in responsive teaching; planning to enact responsive teaching moves, even if those moves are not carried out during the rehearsal, indicates an important step in TCs' development as responsive teachers.

Interviews. Throughout the semester, students participated in three semi-structured interviews conducted by me: once during the first week of the semester (pre-interview), once at midterm (mid-interview), and once during the last week of the semester (post-interview). Table 4 displays the dates and lengths of each interview. See Appendix $\mathrm{H}$ for interview prompts. As with the video annotations, the interviews provided insight into TCs' development of responsive 
teaching through opportunities to discuss their purposes for enacting certain moves at certain times, as well as their perceptions of their own developing practice.

Table 4

Dates and Lengths of Interviews

\begin{tabular}{|c|c|c|c|}
\hline Date & TC & Interview & Length \\
\hline $8 / 15 / 17$ & Julian & Pre & The pre-interviews were \\
\hline $8 / 16 / 17$ & Charity & Pre & $\begin{array}{c}\text { not recorded; exact } \\
\text { lengths are not available. }\end{array}$ \\
\hline $8 / 17 / 17$ & Grace & Pre & $\begin{array}{c}\text { Each interview lasted } \\
\text { approximately 5-15 }\end{array}$ \\
\hline $8 / 17 / 17$ & Bridget & Pre & minutes. \\
\hline $10 / 2 / 17$ & Julian & Mid & $10: 25$ \\
\hline $10 / 2 / 17$ & Grace & Mid & $11: 28$ \\
\hline $10 / 2 / 17$ & Charity & Mid & $12: 21$ \\
\hline $10 / 11 / 17$ & Bridget & Mid & $10: 54$ \\
\hline $12 / 1 / 17$ & Charity & Post & $22: 53$ \\
\hline $12 / 1 / 17$ & Grace & Post & 31:06 \\
\hline $12 / 1 / 17$ & Julian & Post & $56: 19$ \\
\hline $12 / 6 / 17$ & Bridget & Post & $27: 46$ \\
\hline
\end{tabular}

Reflective journal. Finally, I kept a reflective journal as a way to track what aspects of the design were working well, what aspects I should consider changing, and what thoughts I had about TCs' developing practice. I wrote in the journal at least weekly, but usually after each class session that revolved around the cycle of investigation and enactment. One of the challenges of DBR is negotiating the roles of designer and researcher (DBRC, 2003). By keeping a reflective journal of my own noticings and in-the-moment decisions, I aimed to maintain transparency 
while also acknowledging that these multiple roles will impact my analysis of the data (Altheide \& Johnson, 1994; Lincoln \& Guba, 1985). Illustrations of the utility of the journal come later in the chapter.

\section{Methods of Analysis}

Initial video analysis. My analysis began with some initial chunking and coding of the video data using a video analysis software called Studiocode. Once a video has been uploaded to Studiocode, users can use code and label salient moments for further analysis. While similar to Vosaic Connect in some ways, Studiocode includes more robust analysis capabilities.

After each set of rehearsals, I uploaded videos into Studiocode and carried out a first stage of coding that objectively identified who was speaking at any given moment. This initial coding served to help organize later stages of analysis. For example, these objective codes allowed me to see whether students were not speaking because the teacher was instructing or because the teacher had prompted students to take individual or group think time. This is relevant to my research focus because individual and group think time is more likely to be considered responsive than direct instruction by the teacher.

The codes used for this first stage of analysis were: Teacher Talk, Student Talk, Coach Talk, and Ind/Group Work. These instances were then labeled with the IA and the TC's initials for easy identification in later stages of analysis. A sample of the coding window after this initial stage of coding is shown in Figure 3.

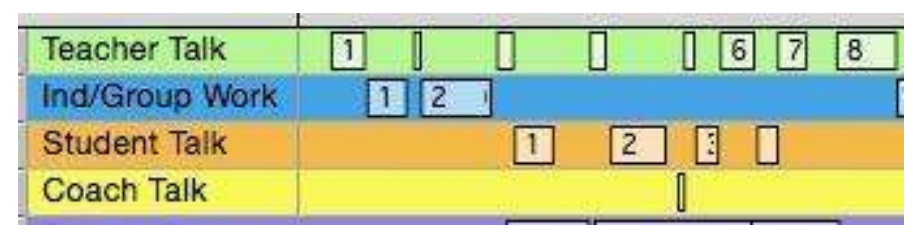

Figure 3. First Stage of Coding in Studiocode 
During a second stage of coding, I grouped instances from the first stage of coding into Student-Teacher (S-T) exchanges that centered on a single idea in an effort to more closely examine the extent to which TCs interacted responsively with students. In some cases, the idea being discussed was strictly mathematical (e.g., the distributive property), while in other cases, a student's solution strategy was the focus of discussion. During the first two IAs, which have finding a solution as a primary goal, some very brief S-T exchanges occurred as the teacher elicited answers from the students. All four IAs involved asking students to reflect as the part of the closure. While these S-T exchanges were also brief, they were each coded as being focused upon a single idea. I made sure that each S-T instance captured enough context to understand the exchange by only watching the S-T instances, which sometimes meant that an S-T instance spanned individual/group think time or a coaching interjection. A sample of the coding window at this stage is shown in Figure 4.

During this second stage of coding, I also grouped instances from the first stage of coding into Coach-Teacher (C-T) exchanges that centered on a single coaching interjection or a question directed to the coach by the teacher. The C-T exchanges varied a bit more in their mathematical focus from asking the teacher to further question a student about a mathematical idea to simply correcting mistakes in recording. Pedagogical considerations related to responsive teaching framed some C-T exchanges, such as when the coach asked the teacher to have another student revoice or when the coach suggested that the teacher give students some think time. Finally, some C-T instances were initiated by the teacher to ask how to proceed in a difficult situation. In these exchanges, the coach provided guidance to help move the IA forward. A sample of the coding window which includes S-T and C-T instances is shown in Figure 4. 


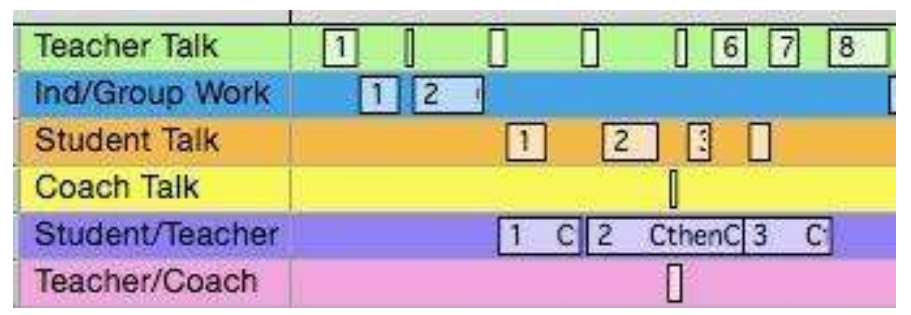

Figure 4. Second Stage of Coding in Studiocode

Labeling S-T instances. In a third stage of video analysis, I labeled the $\mathrm{S}$-T instances based on the individual teaching moves that took place within the instance. A single instance might have multiple move labels. For example, labels such as Elicit Strategy, Ask $S$ if they Agree/Disagree, and T Invites Other Ideas from $S$ could all be applied to a single S-T exchange. The labels and instructions were adapted from the framework by Milewski and Strickland (2016) and my pilot study. Table 5 contains the full set of labeling instructions I used to identify individual teaching moves. See Figure 5 for a sample of the coding window showing the labeled instances.

Table 5

Labeling Instructions for Teaching Moves

\begin{tabular}{|c|c|c|}
\hline Talk Move & Description & Example \\
\hline $\mathrm{T}$ Revoices & $\begin{array}{l}\text { Teacher restates a student idea in his/her } \\
\text { own words, but without adding any } \\
\text { information. }\end{array}$ & $\begin{array}{l}S: 600 \text { is an approximate answer. } \\
T: \text { So then, that's saying that } 31 \times 19 \text { is } \\
\text { not } 600 \text {, but it's close. }\end{array}$ \\
\hline $\mathrm{T}$ Clarifies & $\begin{array}{l}\text { Teacher re-explains a student's idea, while } \\
\text { also adding in details as necessary. }\end{array}$ & $\begin{array}{l}\text { S: The slope is } 1 / 1 \text {. There's a one-to-one } \\
\text { correspondence when you're graphing it. } \\
\text { T: Okay. So let's draw this out. The y- } \\
\text { intercept is at } 7 \text { and you're saying you } \\
\text { would go up one and then over one each } \\
\text { time, right? }\end{array}$ \\
\hline \multirow[t]{2}{*}{ T Repeats } & $\begin{array}{l}\text { Teacher repeats verbatim a student's } \\
\text { offering without alterations. }\end{array}$ & $\begin{array}{l}\text { T: What is one graph that you chose? } \\
\text { S: Graph B. } \\
\text { T: Graph B? Okay. Why? }\end{array}$ \\
\hline & $\begin{array}{l}\text { This often occurs to ensure proper } \\
\text { recording. }\end{array}$ & \\
\hline
\end{tabular}




\begin{tabular}{|c|c|}
\hline Talk Move & Description \\
\hline T Makes Connection & $\begin{array}{l}\text { Teacher draws a connection between } \\
\text { different student ideas/strategies. }\end{array}$ \\
\hline $\begin{array}{l}\text { Ask Different } S \text { to } \\
\text { Revoice }\end{array}$ & $\begin{array}{l}\text { Teacher asks a student to revoice another } \\
\text { student's statement, indicating that the } \\
\text { student may use his/her own words to } \\
\text { reiterate what the original student said. }\end{array}$ \\
\hline & $\begin{array}{l}\text { This may occur to ensure that other } \\
\text { students have understood the statement. }\end{array}$ \\
\hline
\end{tabular}

Ask Different $\mathrm{S}$ to Clarify

Ask $\mathrm{S}$ if Agree/Disagree

T Invites Other Ideas from $S$

T Invites Other Ideas None Elicited

Ask Different $\mathrm{S}$ to Add On

Ask Different $S$ to Make Connection

Ask Original S to Clarify
Teacher asks a student to clarify another student's statement.

This may occur when two students from the same group have the same solution strategy. The teacher may be looking to hear a clearer explanation of the original statement.

Teacher asks an individual student or the whole class whether they agree or disagree with an idea.

Teacher invites additional ideas from a student. The student offers another idea.

Teacher invites additional ideas from a student. The student has no new ideas to offer.

Teacher asks a student to add on to another student's idea.

Teacher asks a student whose strategy/idea is not currently being discussed to draw a connection to another student strategy.

Teacher asks the original student to clarify his/her statement.
T: If we said earlier that one solution means where they intersect, what does that mean if they're laying on top of each other?

S: You're doing inverse operations. $0.9 x$ 100 is 90 , but you can't leave it like that, and if you check $5 \times 18$ you're not going to get 0.9, so you have to remember to divide by 100 so you're undoing operations.

T: Charity, do you want to restate that? $S$ : So, she mentioned that you're using an inverse operation. You start off by multiplying by 100, and then you have to divide by that in the end to eventually get your answer.

T: What similarities did she use [in her solution]?

S: Denominators are multiples of 11.

$T:$ Where is that evident in her problem? Bridget, can you tell us where you applied that?

T: Which of these two methods works best for solving this system?

$S$ : Elimination.

T: (to another student) Do you agree?

T: Is there anything else?

$S$ : There's not a curve.

$T:$ Is there anything else?

S: [Shakes head. No verbal response.]

T: Charity, did you have anything to add? $S$ : We talked about [how] it had to have more than one variable...

T: [reviewing several solutions that have already been discussed] If we have $x=x$, what does that mean? Joey?

S: There are infinite possible answers.

T: What did we do to solve this problem?

$S$ : We added, and then we...

T: What did we add? 


\begin{tabular}{|c|c|c|}
\hline Talk Move & Description & Example \\
\hline & $\begin{array}{l}\text { This signifies a request to perhaps change } \\
\text { the wording or add some detail to make the } \\
\text { statement more easily understood. }\end{array}$ & \\
\hline $\begin{array}{l}\text { Ask Original S to } \\
\text { Repeat }\end{array}$ & $\begin{array}{l}\text { Teacher asks original student to repeat a } \\
\text { statement or idea. }\end{array}$ & \\
\hline $\begin{array}{l}\text { Ask Original S to } \\
\text { Make Connection }\end{array}$ & $\begin{array}{l}\text { Teacher asks the student whose } \\
\text { strategy/idea is being discussed to draw a } \\
\text { connection to another student's strategy. }\end{array}$ & $\begin{array}{l}\text { T: Is this [15 1/6] equivalent to this } \\
\text { [91/6]? } \\
\text { S: Yes. } \\
\text { T: How do we know? } \\
\text { S: You can multiply the } 15 \text { and } 6 \text { which } \\
\text { gives you } 90 \text { and add the } 1 \text { which gives } \\
\text { you 91/6. }\end{array}$ \\
\hline $\begin{array}{l}\text { Ask S a Direct } \\
\text { Question }\end{array}$ & Teacher asks student a direct question. & $\begin{array}{l}\text { T: What do we call this solution method? } \\
\text { S: Substitution. }\end{array}$ \\
\hline $\begin{array}{l}\text { Ask S for Opposing } \\
\text { View }\end{array}$ & $\begin{array}{l}\text { Teacher asks student to explain an } \\
\text { opposing viewpoint. }\end{array}$ & \\
\hline Elicit Answer & Teacher elicits the answer to a problem. & $\begin{array}{l}\text { T: What answer did you get? } \\
\text { S: } 589\end{array}$ \\
\hline Elicit Observation & $\begin{array}{l}\text { Teacher elicits an observation from a } \\
\text { student. }\end{array}$ & $\begin{array}{l}\text { T: What did you notice about this } \\
\text { problem? } \\
\text { S: I noticed that both numbers are prime. }\end{array}$ \\
\hline Elicit Reasoning ${ }^{\mathrm{a}}$ & $\begin{array}{l}\text { Teacher elicits student reasoning about a } \\
\text { particular idea; may occur as an aside } \\
\text { during strategy sharing. }\end{array}$ & $\begin{array}{l}\text { T: You mentioned that, because it's an } \\
\text { inequality, we have to switch the sign } \\
\text { when we divide by a negative coefficient. } \\
\text { Why is that? }\end{array}$ \\
\hline Elicit Reflection & $\begin{array}{l}\text { Teacher elicits a reflection from a student, } \\
\text { usually as a way to close the IA; may } \\
\text { involve a sentence starter. }\end{array}$ & $\begin{array}{l}\text { T: Will you tell me something you learned } \\
\text { today? }\end{array}$ \\
\hline Elicit Strategy & $\begin{array}{l}\text { Teacher elicits the strategy used by a } \\
\text { student to solve the problem. }\end{array}$ & $\begin{array}{l}\text { T: How did you go about solving this } \\
\text { problem? }\end{array}$ \\
\hline $\begin{array}{l}\text { Prompt } S \text { to Take } \\
\text { Think Time }\end{array}$ & $\begin{array}{l}\text { Teacher prompts the group to take a } \\
\text { moment for individual or group think time. }\end{array}$ & $\begin{array}{l}T: \text { What do you think it means to be a } \\
\text { solution to this problem? } \\
S: \text { [No response] }\end{array}$ \\
\hline & $\begin{array}{l}\text { This may occur as part of the IA protocol } \\
\text { or in moments when students seem unsure } \\
\text { of how to respond in a particular moment. }\end{array}$ & $\begin{array}{l}\text { T: Talk to your partner about what it } \\
\text { means to be a solution. }\end{array}$ \\
\hline T Affirms & $\begin{array}{l}\text { Teacher affirms a student's idea using } \\
\text { words or phrases such as, "Yes," or "That's } \\
\text { right." }\end{array}$ & $\begin{array}{l}\text { S: I said there are different methods when } \\
\text { solving an equations. } \\
T: \text { Yes, there is. }\end{array}$ \\
\hline T Answers Question & $\begin{array}{l}\text { Teacher answers a direct question from a } \\
\text { student. }\end{array}$ & $\begin{array}{l}\text { S: Can we subtract } 3 c \text { from both sides? } \\
T: \text { Yes. }\end{array}$ \\
\hline
\end{tabular}




\begin{tabular}{|c|c|c|}
\hline Talk Move & Description & Example \\
\hline T Records & $\begin{array}{l}\text { Teacher records a student's idea on the } \\
\text { board. }\end{array}$ & [No comment required.] \\
\hline T Confirms Recording & $\begin{array}{l}\text { Teacher asks student to verify whether } \\
\text { what has been recorded aligns with what } \\
\text { the student was trying to convey. }\end{array}$ & $\begin{array}{l}\text { S: We discussed that } 9 \text { isn't divisible by } 5 \text {, } \\
\text { but } 90 \text { is, so you can think about } 0.90 \text { to } \\
\text { make it easier to solve. } \\
T: \text { [writes part of response on the board] } \\
\text { Can you say that again? [writes } \\
\text { remainder of response on the board] }\end{array}$ \\
\hline T Corrects & $\begin{array}{l}\text { Teacher makes a correction to a student's } \\
\text { idea. }\end{array}$ & $\begin{array}{l}\text { S: } 5+5 \text { is } 10 . \\
\text { T: Oh, that's a } 15 \text { [written on the board]. } \\
\text { Sorry about that. }\end{array}$ \\
\hline $\mathrm{T}$ further questions $\mathrm{S}$ & $\begin{array}{l}\text { Teacher asks a follow up question based on } \\
\text { something a student has said. }\end{array}$ & $\begin{array}{l}\text { S: I noticed that the graph of this equation } \\
\text { would be less steep than all the others. } \\
\text { T: How did you know it would be less } \\
\text { steep? } \\
\text { S: Because it has the smallest slope. }\end{array}$ \\
\hline
\end{tabular}

${ }^{\mathrm{a}}$ Elicit Reasoning was not used if no student offered reasoning. For example, if a teacher asked, "Are there any other reasons we might classify this as a function?" and no one responded, then this label was not used.

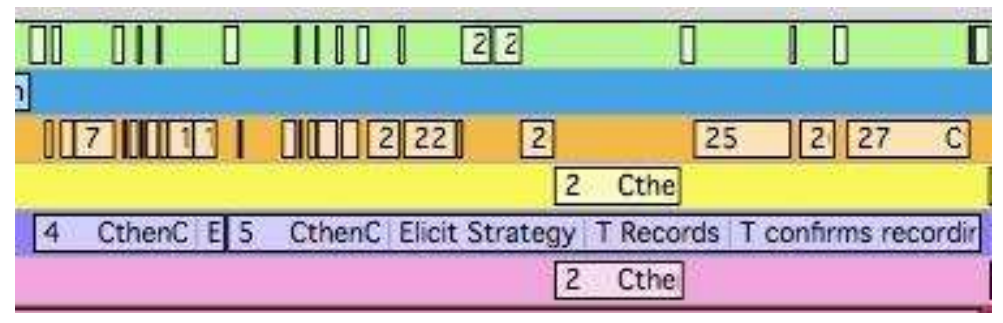

Figure 5. Sample of Labeled Instances in Studiocode

The process of labeling the S-T instances was iterative. As I re-watched the instances, new teaching moves and patterns emerged, which occasionally resulted in editing an existing label or creating a new one. Any additions or changes to labels were followed by another review of all S-T instances to ensure the labels had been applied correctly. For example, one label that emerged during my analysis was Ask $S$ if they Agree/Disagree. It was not until I watched the TCs' school enactments, that I noticed TCs using this move; it did not capture my attention during the rehearsals in methods. However, upon review of all S-T instances, I found that TCs 
did occasionally enact this move during their rehearsals. As a result, labels were updated accordingly.

As another example, the label T Invites Other Ideas from $S$ evolved over the course of the study. It began as a way to keep track of how often TCs asked questions like, "Anything else?" or "Any questions?" A whole-class discussion with the TCs led me to the decision to label only the instances when a new idea was actually elicited as a result of the TCs' invitation. During my analysis, however, I realized that it would also be useful to track instances in which no new ideas were elicited, which led to the creation of a new label: T Invites Other Ideas - None Elicited. The final labeling scheme is found in Table 5.

Labeling C-T instances. Finally, using a classification system similar to Lampert and colleagues (2013), I labeled the C-T instances based on the substance and structure of the exchange. The substance of a C-T instance described what was discussed during the exchange and usually focused on a teaching move (e.g., C Asks T to Further Question S, C Asks T to Let S Revoice). The structure of the exchange served as more of a description of how the exchange was initiated (e.g., T asks $C$ a question, $C$ Compliments T). Not every C-T instance was given a structure label; the absence of a structure label indicated that the coach was providing directive feedback during the exchange. As with the S-T instances, a single instance might include multiple substance labels, but this occurred less frequently with the C-T instances. Most of the labels were recurring, but for several instances, the labels I created did not necessarily capture the content of the instance. In those cases, I recorded the substance of the exchange using the Text feature in Studiocode, which allows space for a longer description that will be stored with the instance for future review. Table 6 contains the labeling instructions I used to classify coaching interjections. 
Table 6

Labeling Instructions for Coaching Interjections

\begin{tabular}{ccc}
\hline $\begin{array}{c}\text { Coaching } \\
\text { Interjection }\end{array}$ & Description & Example \\
\hline
\end{tabular}

C Asks T to

Highlight Math

Idea

\section{Asks T to \\ Ask Open \\ Questions}

Coach asks the teacher to make a mathematical idea more explicit.
Coach asks the teacher to phrase questions to provide opportunities to hear student reasoning (rather than leading students to a term or idea).

Coach asks the teacher to rephrase the question to make it easier to understand.
C Asks T to

Further Question S

$\mathrm{C}$ asks $\mathrm{T}$ to Let

$S$ revoice

C Comments on T's Recording

C Suggests that T Provide Think Time
$C:$ There are some things that Bridget did that we should just make explicit as to why we can do that.

T: Oh, okay.

C: She moved the decimal from 0.9, she turned that into 90, and then at the end, she had 18 but she made it 0.18. A question you can pose is, "Why can we do that?" or "Can we do that?"

T: So, can somebody tell me why we can...move the decimal to make this 90 ...but we can move the decimal back?

T: So now, since we have the definition, I want you to rethink $y / x=2.5$ with your partner.

C: I'm going to have you phrase that a little differently, because when you say, "rethink it," you're already telling us, "That was wrong." ...So maybe say, "Will you look at them and see if there are any that need to trade places?" Leave it really open like that, so then that leaves it up to us to decide between all six.

T: So this asymptote. She said that it never crosses that point. Let's consider the other graphs as well.

C: I'm not clear on what we're supposed to be doing. Do you mean think about whether the other graphs have asymptotes or not?

T: Uh-uh. [No.]

C: Okay. I think I'm just confused about your question. Can you say one more time what you want us to think about?

T: I want you to compare all of them and see differences in the graphs.

C: One thing that might be important to follow up on here is how she knew that $4 / 11$ was closest to $1 / 2$.

T: Okay. So how did you know that $4 / 11$ was closest to $1 / 2$ ?

C: Why don't we have someone re-explain what Bridget did? Just to make sure they understand everything she did.

T: Grace, can you explain what Bridget just did?

C: You have to be really careful about using horizontal equals because right now you have $31 \times 20=620-31=589$.

T: So what do you think it means to be a solution to this problem...looking at those answers and your answers from before? 
decide how to respond to a question or prompt.
Coach helps the teacher manage the technology.

Coach reminds the teacher of details in the IA protocol.

T Asks C a Question

[No response from students after several seconds.] C: If you notice that everyone is not sure what to say, you can say "Talk to your partner about what makes something a solution to an equation." Give them some group think time.

C: On the next screen, you'll have a bigger space to write.

T: Talk with the person sitting next to you and discuss which one you picked.

$C$ : Be sure to emphasize to us to have a reason, too.

Because students will sometimes pick one without having a good reason.

After an explanation (using only numbers) of why to switch the inequality when multiplying by a negative number:

T: I'm not sure how to go about introducing it with the variable.

C: ... you could check this inequality, to make sure the values will make the initial inequality true. That might help move this conversation, which is strictly numerical, into one that involves a variable.

Focused data analysis. Once all instances had been labeled consistently, I created a database of all 24 videos in Studiocode. The database compiled all codes and labels from each of the individual TC videos. (As an example, Figure 5 above shows the coded instances from a single rehearsal.) The database allowed me to create a matrix which organized a count of all codes and labels. Figure 6 displays a sample of the matrix as it appeared in Studiocode. Table 7 displays the matrix in full, though I removed the columns related to my initial stage of coding (i.e., Teacher Talk, Individual/Group Work, Student Talk, and Coach Talk) because they had no direct impact on the matrix trends I used to further my analyses. Together, the database and matrix served as primary data sources in addressing my three research questions. Other data sources (see Table 8) provided additional means for pursuing answers to each question. In the sections below, I describe my analysis methods as they relate to each question. 


\begin{tabular}{|c|c|c|c|c|c|c|c|c|c|c|c|c|}
\hline & 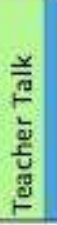 & 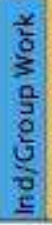 & 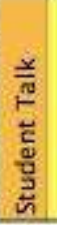 & $\begin{array}{l}\frac{x}{\mathrm{~m}} \\
\stackrel{5}{\mathrm{c}} \\
\text { gू } \\
\mathrm{g}\end{array}$ & 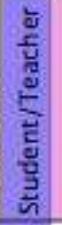 & 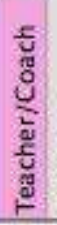 & 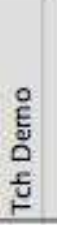 & 8 & 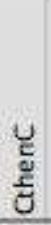 & 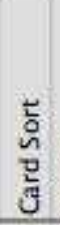 & §̊ & $\frac{8}{8}$ \\
\hline Ask different $S$ to add on & & & & & 5 & & & & 1 & 3 & 9 & 1 \\
\hline Ask different $S$ to clarify & & & & & 4 & & 1 & 1 & 1 & 1 & 1 & 3 \\
\hline Ask different S to Make Connection & & & & & 4 & & & 3 & 1 & & 1 & 1 \\
\hline Ask different $S$ to Revoice & 1 & & & & 31 & & & 5 & 6 & 1 & 9 & 9 \\
\hline Ask $S$ if agree/disagree & & & & & 17 & & & & & & & 7 \\
\hline Prompts students to take ind/g... & 2 & & & & 11 & 1 & & 22 & 25 & 19 & 27 & 17 \\
\hline T clarifies & & & & & 15 & & & 6 & 7 & 7 & 13 & 4 \\
\hline T confirms recording & & & & & 19 & & & 1 & 4 & 5 & 10 & \\
\hline$T$ further questions S & & & & & 68 & & & & & & & \\
\hline$T$ invites other ideas - none eicited & & & & & 51 & & & & & & & \\
\hline$T$ invites other ideas from $S$ & & & & & 18 & & & & 1 & 5 & 21 & \\
\hline T makes Connection & & & & & 7 & & & 4 & & 2 & 6 & 1 \\
\hline$T$ repeats & & & & & 70 & & 5 & 10 & 8 & 16 & 10 & 20 \\
\hline T Revoices & 1 & & & & 30 & & 1 & 8 & 8 & 7 & 15 & 6 \\
\hline C asks $T$ to ask open questions & & & & 5 & & 7 & & 3 & & 1 & 2 & \\
\hline C asks $T$ to clarify question & & & & 4 & & 6 & & 3 & & 1 & 2 & \\
\hline $\mathrm{C}$ asks $\mathrm{T}$ to further question $\mathrm{S}$ & 1 & & & 4 & & 11 & & 1 & 4 & 4 & 3 & \\
\hline $\mathrm{C}$ asks $\mathrm{T}$ to highlight math idea & & & & 6 & & 9 & & 3 & 2 & 1 & 1 & \\
\hline C asks $\mathrm{T}$ to let $\mathrm{S}$ revoice & & & & 4 & & 6 & & 1 & 2 & & 3 & \\
\hline C comments on IA protocol & & & & 6 & & 20 & & 3 & 1 & 12 & 2 & \\
\hline C comments on T's recording & & & & 9 & & 19 & & 6 & 4 & 2 & 5 & \\
\hline C compliments $T$ & & & & 3 & & 8 & & 3 & & 2 & 3 & \\
\hline $\mathrm{C}$ suggests that $\mathrm{T}$ give student $\mathrm{t} . .$. & & & & 5 & & 8 & & 5 & 1 & 3 & & \\
\hline T asks $C$ a question & & & & & & 10 & & 2 & & 7 & 3 & \\
\hline \multirow{2}{*}{ (1) } & 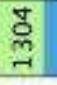 & $\stackrel{\circ}{\circ}$ & $\vec{\otimes}$ & $\stackrel{1}{\rightarrow}$ & $\stackrel{\infty}{\stackrel{N}{N}}$ & $\stackrel{8}{\circ}$ & 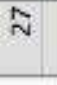 & m & के & $\underset{-}{g}$ & $\$$ & $\frac{\mathscr{R}}{\kappa}$ \\
\hline & 5 & & & & & & & & & & & \\
\hline
\end{tabular}

Figure 6. Matrix from Studiocode Database 
Table 7

S-T Instances

\begin{tabular}{|c|c|c|c|c|c|}
\hline Teaching Move & Total & Julian & Bridget & Charity & Grace \\
\hline T Revoices & 30 & 9 & 8 & 5 & 8 \\
\hline $\mathrm{T}$ Clarifies & 15 & 4 & 4 & 1 & 6 \\
\hline T Repeats & 70 & 27 & 12 & 12 & 18 \\
\hline $\mathrm{T}$ makes Connection & 7 & 2 & 1 & 1 & 3 \\
\hline Ask Different $S$ to Revoice & 31 & 5 & 4 & 14 & 8 \\
\hline Ask Different $S$ to Clarify & 5 & 1 & 0 & 3 & 1 \\
\hline Ask $S$ if Agree/Disagree & 10 & 0 & 4 & 2 & 4 \\
\hline $\mathrm{T}$ Invites Other Ideas from $\mathrm{S}$ & 17 & 7 & 1 & 4 & 4 \\
\hline Ask Different $\mathrm{S}$ to Add On & 6 & 1 & 1 & 2 & 2 \\
\hline Ask Different S to Make Connection & 4 & 0 & 3 & 0 & 1 \\
\hline Ask Original S to Clarify & 69 & 15 & 19 & 22 & 13 \\
\hline Ask Original S to Repeat & 22 & 9 & 7 & 2 & 4 \\
\hline Ask Original S to Make Connection & 4 & 0 & 2 & 0 & 2 \\
\hline Ask S a Direct Question & 89 & 9 & 29 & 27 & 23 \\
\hline
\end{tabular}




\begin{tabular}{|c|c|c|c|c|c|}
\hline Teaching Move & Total & Julian & Bridget & Charity & Grace \\
\hline Ask $\mathrm{S}$ for Opposing View & 6 & 1 & 1 & 2 & 0 \\
\hline Elicit Answer & 91 & 17 & 24 & 23 & 25 \\
\hline Elicit Observation & 13 & 3 & 4 & 3 & 3 \\
\hline Elicit Reasoning & 94 & 21 & 27 & 23 & 21 \\
\hline Elicit Reflection & 47 & 13 & 13 & 14 & 7 \\
\hline Elicit Strategy & 28 & 6 & 4 & 12 & 6 \\
\hline Prompts Students to Take Ind/Group Think Time & 11 & 1 & 1 & 3 & 6 \\
\hline Prompts Students to Take Think Time & 0 & 0 & 0 & 0 & 0 \\
\hline T Affirms & 30 & 10 & 3 & 7 & 10 \\
\hline T Answers Question & 10 & 0 & 4 & 3 & 3 \\
\hline T Records & 198 & 41 & 62 & 45 & 48 \\
\hline T Confirms Recording & 19 & 8 & 6 & 0 & 4 \\
\hline T Corrects & 2 & 0 & 0 & 2 & 0 \\
\hline T Further Questions S & 68 & 32 & 28 & 48 & 28 \\
\hline
\end{tabular}

Note. In the Studiocode matrix, the totals often do not represent the correct sum. Matrix counts may differ slightly from the actual counts of moves found later in this dissertation. The matrix was used as a starting point, rather than means of determining the actual number of enactments of each move. 
Table 8

Research Questions, Data Sources, Methods of Analysis

\begin{tabular}{|c|c|c|}
\hline Research Question & Data Source(s) & Analyses \\
\hline \multirow{5}{*}{$\begin{array}{l}\text { How do TCs develop skill in } \\
\text { teaching mathematics } \\
\text { responsively within a } \\
\text { methods course in which } \\
\text { cycles of investigation and } \\
\text { enactment are a central } \\
\text { pedagogy? }\end{array}$} & Video - rehearsals & Examined S-T instances of responsive teaching practices \\
\hline & $\begin{array}{l}\text { Video } \\
\text { annotation assignment }\end{array}$ & $\begin{array}{l}\text { Examined TC perceptions of their own enactment of responsive teaching practices, } \\
\text { the language they used, and the practical tools they discussed }\end{array}$ \\
\hline & Planning materials & $\begin{array}{l}\text { Examined TCs' anticipated student responses and protocol notes to look for evidence } \\
\text { of their plans to enact responsive teaching }\end{array}$ \\
\hline & $\begin{array}{l}\text { Interviews } \\
\text { (Pre, Mid, Post) }\end{array}$ & $\begin{array}{l}\text { Examined TC perceptions of their own enactment of responsive teaching practices, } \\
\text { the language they used, and the practical tools they discussed }\end{array}$ \\
\hline & Reflective Journal & $\begin{array}{l}\text { Looked for patterns/development for individual TCs and for all TCs as a group. } \\
\text { Identified other factors that might impact TC development }\end{array}$ \\
\hline \multirow{4}{*}{$\begin{array}{l}\text { How does coaching in } \\
\text { methods affect TCs' } \\
\text { responsive teaching? }\end{array}$} & Video - rehearsals & Examined C-T instances involving responsive teaching \\
\hline & $\begin{array}{l}\text { Video } \\
\text { annotation assignment }\end{array}$ & $\begin{array}{l}\text { Examined TC perception of the effect of coaching on their enactment of responsive } \\
\text { teaching practices, the language they used, and the practical tools they discussed }\end{array}$ \\
\hline & Interviews (Mid, Post) & $\begin{array}{l}\text { Examined TC perception of their own enactment of responsive teaching practices, } \\
\text { the language they used, and the practical tools they discussed }\end{array}$ \\
\hline & Reflectiv & $\begin{array}{l}\text { Looked for patterns/development for individual TCs and for all TCs as a group. } \\
\text { Identified other factors that might impact TC development }\end{array}$ \\
\hline \multirow{4}{*}{$\begin{array}{l}\text { To what extent do TCs teach } \\
\text { responsively in school } \\
\text { settings? }\end{array}$} & Video - classroom & Examined S-T instances of responsive teaching practices \\
\hline & $\begin{array}{l}\text { Video } \\
\text { annotation assignment }\end{array}$ & $\begin{array}{l}\text { Examined TC perception of their own enactment of responsive teaching practices, } \\
\text { the language they used, and the practical tools they discussed }\end{array}$ \\
\hline & Planning materials & $\begin{array}{l}\text { Examined TCs' anticipated student responses and protocol notes to look for evidence } \\
\text { of their plans to enact responsive teaching }\end{array}$ \\
\hline & Interviews (Post) & $\begin{array}{l}\text { Examined TC perception of their own enactment of responsive teaching practices, } \\
\text { the language they used, and the practical tools they discussed }\end{array}$ \\
\hline
\end{tabular}


Research question 1. To examine how TCs developed skill in teaching mathematics responsively within the methods course, I looked to the matrix to identify any trends related to the S-T instances and individual teaching moves from the rehearsals that occurred in methods. Note that S-T instances from the school enactments were not considered as part of these analyses. Labels that seemed to occur especially often (e.g., T Repeats) or especially infrequently (e.g., T Makes Connection) drew my attention first. I also sorted labels (moves) based on similarities. For example, I grouped moves in which the teacher articulated mathematical ideas (i.e., T Revoices, $T$ Clarifies, or T Repeats) to compare how moves were used with similar, though not identical, purposes. From this process, my analysis focused on four trends.

Matrix trends. The first trend I investigated was around a set of moves in which the teacher articulated a mathematical idea that had originated with a student (i.e., T Revoices, $T$ Clarifies, or $T$ Repeats). This was interesting to me for two reasons. First, there are key differences in how the teacher articulates a student's idea. Second, TCs used the move T Repeats much more often, which raises questions about how TCs are using these different moves. The second trend I investigated centered on the move T Makes Connection. This was one of the least frequently enacted moves during the study, which is consistent with the results of my pilot study. Thus, the infrequency of T Makes Connection raised questions about why TCs in two different courses enacted this move so sparingly - is it a move that is generally more difficult for TCs to enact? If so, why might that be? The third trend I investigated focused on a group of teaching moves that would seem to orient students to one another's thinking (i.e., Ask Different $S$ to Revoice, Ask Different $S$ to Clarify, and Ask $S$ if they Agree/Disagree). There was inconsistency in the use of these moves across TCs (i.e., certain TCs used these moves frequently, while others hardly used these move at all), which raises questions about how and when TCs were enacting 
these moves, as well as why they had tendencies toward particular moves that oriented students to one another's thinking. The final trend I investigated looked at moves when the TC invited additional ideas from students. I examined instances of $T$ Invites Other Ideas from $S$ and $T$ Invites Other Ideas from $S$ - none elicited more closely to determine whether there were common characteristics of moves that successfully elicited additional student ideas, as opposed to the moves when no new student ideas were elicited. Were successful moves more likely to occur in certain IAs or in certain moments of each IA (i.e., the introduction or the closure)? A better understanding of these trends might provide guidance to TEs and TCs about ways to invite additional ideas purposefully and therefore, responsively.

Analysis of matrix trends. To further investigate each of these four trends, I created merged rows for the relevant individual teaching moves in the Studiocode database. A merged row acts as a filter; it places all instances from a coding row with a particular label into a database row of their own for easier analysis. For example, one of my merged rows extracted all S-T instances that had been labeled Ask Different $S$ to Revoice. (A sample of the database coding window in Figure 7 shows the merged rows below the original coding rows.) I watched the instances in each merged row sequentially and recorded analytic memos using the Text feature in Studiocode, which stores each memo with its corresponding instance. The content and degree of detail in the analytic memo was dependent upon the teaching move being examined. In general, I considered whether the teaching move was used responsively and recorded the rationale for my decision. 


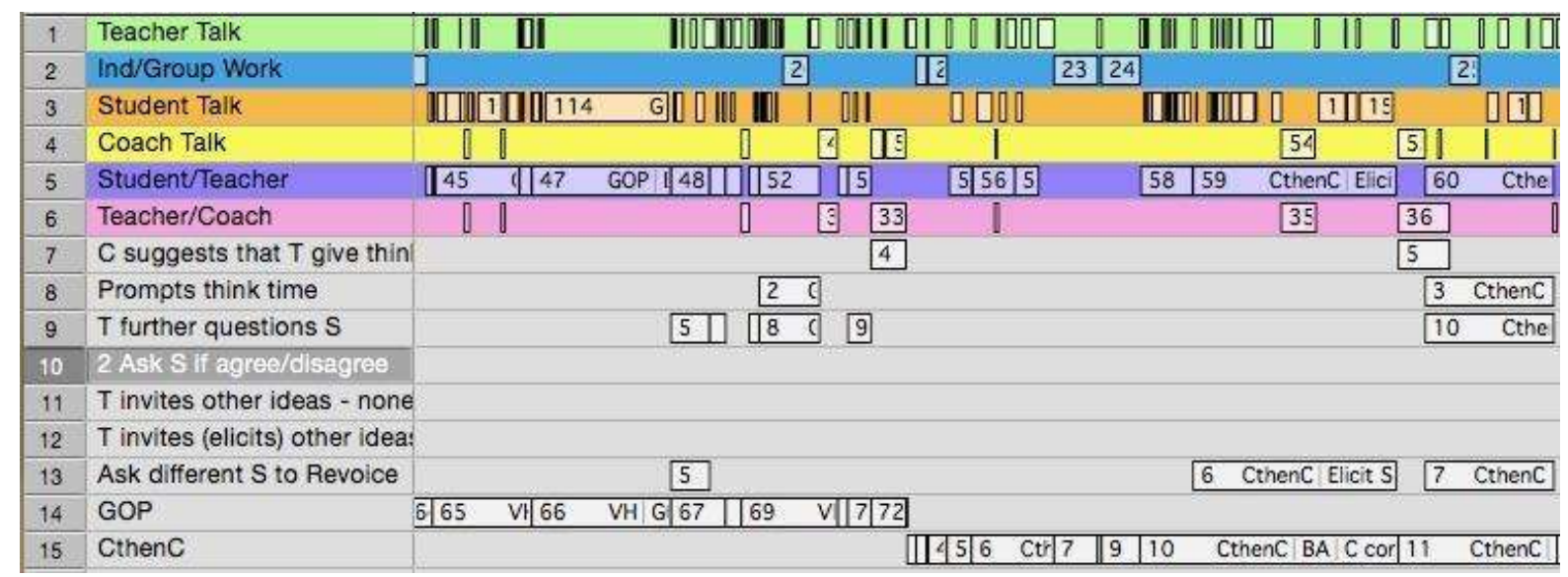

Figure 7. Sample of Studiocode Database

Deciding whether a particular teaching move was used responsively was not entirely straightforward. Considering the moves at varying levels of grain size helped me tease apart different aspects of responsiveness. A single move (smaller grain size) was characterized as responsive based primarily on the enactment of the move, with little or no consideration of the intent or result of the move. For example, Ask a Different $S$ to Revoice was considered a responsive move because, ideally, the intent behind this move would be to orient students to each other's ideas. During my initial phases of labeling S-T instances, the move was labeled simply if it happened at all—at that point, I did not consider the TC's intent or the result of the move.

Simply because a move is designed with a particular purpose in mind, however, does not ensure that TCs will enact the move with the same intent. It was not until the conclusion of the semester, when the database was created and I began watching S-T instances involving specific teaching moves sequentially, that I began to examine the overall responsiveness of the move in the context of the entire S-T exchange. This required looking at sequences of moves (larger grain size), which included a consideration of how the student responded and how (or if) the teacher incorporated that response as part of their instruction. Examining the responsiveness of teaching moves at varying grain sizes allowed for a more robust analysis. 
Consider the following example from a rehearsal of Which One Doesn't Belong. After eliciting several characteristics of a graph that make it different from the other three, the rehearsing teacher (Bridget) asked Julian (playing the role of a student) if he had anything to add to the list. When he said he had nothing to add, only then did she ask him to revoice — a move which, on the surface, seems like it should be responsive because it implies that the teacher is working to orient students to one another's ideas. In this case, however, the intent behind the move seemed to be to ensure that each student had an opportunity to speak, rather than to orient students to one another's ideas. Furthermore, although Julian did repeat why the chosen graph was different from the others, he simply read from the board the list of observations that had been recorded; he did not put the observations into his own words. Therefore, even though the teacher enacted what seemed on the surface to be a single responsive teaching move, this exchange was considered unresponsive as a whole because there was no evidence that Julian had come to a greater understanding of other students' ideas through his revoicing.

Once S-T instances were characterized as responsive or unresponsive, I created tables to compare the frequency and responsiveness of related moves (e.g., $T$ Clarifies, $T$ Repeats, and $T$ Revoices). These tables were organized according to IA, TC, or both, which allowed me to draw conclusions as to whether the frequency of a particular move was dependent upon the IA or the TC.

Other data sources. TCs' planning materials were examined through content analysis for evidence of development in responsive teaching. On the pre-planners, looking at the anticipated responses recorded by TCs provided some indication of how deeply they considered student responses before the enactment of the IA (e.g., Did TCs consider conceptual misunderstandings or were their considerations largely procedural, such as forgotten negative signs and minor 
arithmetic errors?). TCs' protocol notes also provided some insight into their development as responsive teachers through the inclusion (or exclusion) of notes related to enacting certain teaching moves at particular times. When present, these notes suggested that TCs were making an effort to enact responsive teaching moves purposefully.

TCs' video annotations and interview data were analyzed through open coding. After the conclusion of the semester, I placed TCs' interview responses (based on my written interview notes and/or recordings) in a table which organized them by TC and by interview (Pre, Mid, or Post). I then looked across the three different interviews for each TC to see how they talked about the teaching moves I had identified as significant. TCs' perceptions of those moves and their purposes would provide an indication of their understanding of how the moves may be used to support responsive teaching.

Video annotations from Vosaic Connect were downloaded to a spreadsheet and organized into a workbook. Each worksheet contained annotations from a different IA, organized by TC. Through this analysis, I was able to uncover evidence of TCs' perceptions of their enactments of responsive teaching moves. For example, TCs were asked to identify moments when they focused the discussion on student reasoning. In these annotations, TCs mentioned moves that they thought they had used well or that they wish they would have used. As with the interviews, this provided an indication of TCs' perceptions and understandings of responsive teaching and the moves that support that work.

Open coding of my reflective journal revealed my perceptions of TCs' evolving use of responsive teaching moves. For example, I was particularly attentive to TCs' developing use of the move Ask Different $S$ to Revoice. The results of this analysis provided insights into differences and similarities between TCs' perceptions and my perceptions, which suggested 
some potential revisions for the design of future iterations of the methods course. These revisions are discussed in Chapter 5.

Taken together, these considerations led me to three overarching themes in response to RQ1: teaching moves that were consistently enacted responsively by TCs, teaching moves that are positioned as responsive in the literature but were not always enacted in responsive ways by TCs, and teaching moves that were enacted habitually, rather than with any apparent purpose. These themes will be discussed in greater detail in Chapter 4.

Research question 2. In examining how coaching in methods affected TCs' responsive teaching, I looked to the C-T rows of the matrix to identify coaching trends (See Table 9). I first took note of labels that seemed to occur especially often or especially infrequently. I also used the S-T rows matrix to compare the frequency of labels that might have been related, such as Ask Different $S$ to Revoice and C Asks T to Let S Revoice. Trends of interest related to coaching did not come solely from the matrix; I also gave some consideration to the trends I found related to the first research question and how coaching might have impacted TCs' enactment of certain moves. In particular, I looked at how often TCs were enacting the moves on their own as opposed to enacting the same moves in response to a request from the coach. 
Table 9

\section{C-T Instances}

\begin{tabular}{lccccc}
\hline & $\begin{array}{c}\text { Teacher- } \\
\text { Coach }\end{array}$ & GOP & CthenC & $\begin{array}{c}\text { Card } \\
\text { Sort }\end{array}$ & WODB \\
\hline C Asks T to Ask Open Questions & 7 & 3 & 0 & 1 & 2 \\
C Asks T to Clarify Question & 6 & 3 & 0 & 1 & 2 \\
C Asks T to Further Question S & 11 & 1 & 4 & 4 & 3 \\
C Asks T to Highlight Math Idea & 7 & 3 & 2 & 1 & 1 \\
C Asks T to Let S Revoice & 6 & 1 & 2 & 0 & 3 \\
C Comments on IA Protocol & 20 & 3 & 1 & 12 & 2 \\
C Comments on T's Recording & 19 & 6 & 4 & 2 & 5 \\
C Compliments T & 8 & 3 & 0 & 2 & 3 \\
C Helps T with Technology & 3 & 0 & 2 & 1 & 0 \\
C Suggests that T Give Students Think Time & 8 & 5 & 1 & 3 & 0 \\
T Asks C a Question & 10 & 2 & 0 & 7 & 3 \\
\hline
\end{tabular}

Note. In the Studiocode matrix, the totals often do not represent the correct sum. Matrix counts may differ slightly from the actual counts of moves found later in this dissertation. The matrix was used as a starting point, rather than means of determining the actual number of enactments of each move. 
Matrix trends. I first investigated the coaching interjection C Asks T to Let S Revoice. One of the trends related to the first research question showed that TCs enacted Ask Different $S$ to Revoice more frequently than the other orienting moves. As a result, I considered the extent to which coaching played a role in TCs' enactment of that move. The second and third trends I investigated centered on C Comments on T's Recording and C suggests T give S Think Time. I examined these instances more closely to determine the extent to which my interjections were focused on responsive teaching because it was unclear from the matrix why I made these interjections. For example, C Comments on T's Recording was the second-most occurring coaching interjection, but if my interjections were focused more on TCs' handwriting than on whether the TC had accurately represented student ideas, then that large number of interjections would not have conclusively contributed to TCs' development of responsive teaching. The matrix revealed that my use of $C$ Suggests $T$ give S Think Time decreased over the course of the semester, which raised questions about whether TCs did this more frequently on their own and in responsive ways. This led me to compare both $C$ Comments on T's Recording and $C$ suggests $T$ give S Think Time with the frequency of their corresponding S-T instance labels, T Records and T Prompts $S$ to Take Think Time, to determine the extent to which TCs were enacting these moves on their own. Finally, I noticed that CAsks T to Further Question $S$ was the third most frequently occurring C-T label, which again raised questions about the substance of these interjections and whether they were centered on responsive teaching. For each of the four trends, I also considered whether interjections from early in the semester seemed to impact TCs' responsive teaching as the semester progressed.

Analysis of matrix trends. As with the analysis of the first research question, I created merged rows in the database, watched the instances from each row sequentially, and recorded 
analytic memos related to the substance of each C-T exchange. In particular, I noted whether the exchange was focused on responsive teaching, which was the lens through which I viewed my coaching responsibilities. The consideration of grain size was less pertinent in deciding whether the C-T exchanges were focused on responsive teaching because these instances did not involve a sequence of responsive teaching moves, as was often the case with the $\mathrm{S}$-T instances. Therefore, the determination of whether or not a C-T instance was focused on responsive teaching relied heavily upon the substance of the exchange.

Once C-T instances had been characterized according to whether they were focused on responsive teaching, I then created tables to display the frequency of coaching interjections with each substance label and whether they were focused on responsive teaching. The table is organized by substance and structure, which allowed me to draw conclusions related to the responsiveness of $\mathrm{C}-\mathrm{T}$ instances with particular substance labels as they related to the structure of the C-T instances.

Other data sources. TCs' video annotations and interview data were examined in ways similar to those discussed in reference to the first research question. Analyses related to the second research question, however, involved looking for trends specifically related to TCs' perceptions and attitudes toward coaching and the coached rehearsals. During the mid- and postinterviews, I specifically asked TCs to describe their thoughts and feelings related to the coached rehearsals and the ways they were impacting their development as teachers. TCs' responses to this question were fairly general in nature.

More detail was revealed through analysis of their video annotations. TCs were asked to identify challenging moments in their rehearsals, as well as moments when they either felt supported or felt they could have used more support in their teaching. It was in these annotations 
that TCs discussed particular moves and provided a sense of how they viewed my feedback. For example, although not explicitly stated, some of the TCs' video annotations seem to indicate that they perceived some of my coaching interjections as more punitive than instructive.

Open coding of my reflective journal revealed my perceptions of the coaching and the way it was impacting TCs' development. In particular, I made note of what I saw as my own shortcomings as a coach, which informs my analysis of TCs' responsive teaching. For example, I regularly wrote about my own flawed enactments of Ask Different $S$ to Revoice, which likely explains why I was especially attentive to TCs' enactment of that move.

Looking across these trends led me to first examine the extent to which each coaching interjection was focused on responsive teaching - a necessary first step in order to determine whether TC development in responsive teaching could reasonably be attributed to coaching. Then, I compared four of the most frequently occurring types of coaching interjections with their corresponding S-T instances (when TCs enacted the moves without direction from the coach) to determine whether coaching helped TCs to enact these moves more responsively on their own over time. Finally, using TCs' video annotations and interviews, I examined their perceptions of instructional coaching and how those perceptions may have impacted the ways they took up coaching feedback. These themes will be discussed in more detail in Chapter 4.

Research question 3. In considering the extent to which TCs teach responsively in school settings, I used the matrix a little differently. Instead of looking at primarily the S-T column, I also examined the School GOP column to gain a sense of how frequently the TCs enacted teaching moves in schools when compared with their Teaching Demonstrations or other IA rehearsals in methods. Three trends emerged from this analysis. 
Matrix trends. The first trend I investigated related to a comparison of TCs' School GOP enactments with their Teaching Demos. This comparison revealed that TCs enacted some responsive teaching moves (i.e., T Prompts $S$ to Take Think Time and Ask Different S to Revoice) in schools that were not enacted at all during their Teaching Demonstrations. I looked at TCs' enactments of these moves more closely to determine the extent to which they were used responsively in schools. The second and third trends involved a comparison of TCs' enactments of the IAs in methods and School GOP. The matrix showed that some moves were enacted less frequently (e.g., Ask Different $S$ to Revoice), while other moves (e.g., Ask $S$ if they Agree/Disagree) were enacted more frequently during the school enactments. This raises questions about the extent to which TCs used either group of moves responsively in the school setting when compared to their enactments of these moves in methods.

Analysis of matrix trends. To further investigate these three trends, I looked to the merged rows in the database. As with the analysis of the first research question, I watched the S-T instances from the enactments of School GOP in each merged row sequentially, recorded analytic memos in Studiocode, and considered whether each instance involved a responsive use of the teaching move being examined. Finally, I compared the responsiveness of moves used during School GOP with the responsiveness of moves during the IAs in methods and the Teaching Demos to better examine the extent to which TCs' responsive teaching may have changed in the school setting.

As a final stage of video analysis for TCs' school enactments, I downloaded video annotations from Vosaic Connect and then imported them into the individual Studiocode video files. A sample of the coding window at this stage of analysis is shown in Figure 8. The first six rows are my codes and labels; the bottom four rows show moments that the TC identified. This 
made it convenient to compare TCs' perceptions to my own perceptions around particular instances, to investigate these moments with a greater level of depth. This was only possible for instances on which TCs chose to comment; they were not prompted by me to write about any instance in particular.

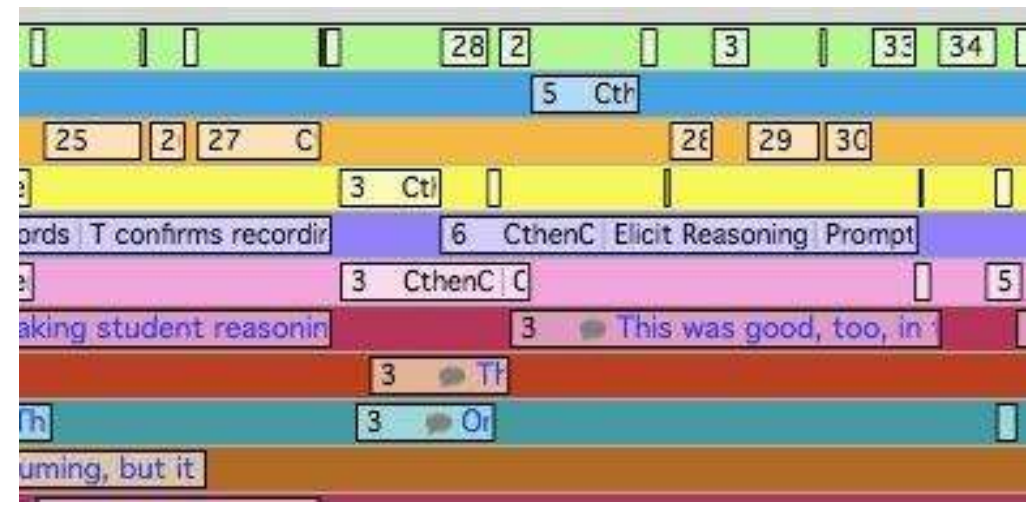

Figure 8. My Coding (Rows 1-6) and TC Video Annotations from Vosaic Connect (Rows 7-10) I chose to include this stage of analysis specifically for TCs' school enactments because I was unfamiliar with the school contexts. After coding the school enactments and reviewing TCs' video annotations, I realized that moves I had characterized as generally unresponsive or unproductive were sometimes a response to a specific attribute of the school setting. For example, Charity used the move Ask Different $S$ to Revoice excessively with varied success during her School GOP IA. Upon reading her video annotations, I found that the resource teacher in the room with her often had students restate the comments of others, which helped explain why she used that move so frequently. Importing the annotations into Studiocode facilitated the direct comparison of my and the TCs' perceptions of the school enactment. Once S-T instances from School GOP were characterized as responsive or unresponsive, I added this data to the tables I created for the first research question. These amended tables revealed that there were few trends related to TCs' enactments of teaching moves that applied to all TCs; their responsive 
teaching in schools varied significantly. As a result, I decided to build vignettes for each TC to provide a more general sense of their school enactments. Each vignette includes a commentary in which I discuss enactments of teaching moves that either stood out across all four school enactments (e.g., all TCs enacted T Prompts $S$ to Take Think Time) or that stood out for a particular TC (e.g., Bridget's enactments of Ask S if they Agree/Disagree were more frequent and more responsive in schools than in methods).

Other data sources. TCs' planning materials, video annotations, and interview data were examined in ways similar to those discussed in reference to the first research question. Similar to the analysis of RQ1, TCs' planning materials were examined for evidence of plans to enact responsive teaching, both through the TCs' anticipated student responses and through the notes written on their protocols as reminders of responsive teaching moves to enact in specific moments. When present, these notes suggested that TCs were making an effort to enact responsive teaching moves purposefully, even in the school setting.

TCs' video annotations revealed significant detail and insight into their perceptions of their school enactments. By looking at moments that TCs identified as focusing on student reasoning or as particularly challenging, I gained an understanding of how they viewed responsive teaching; their annotations mentioned particular moves and the reasons they chose to enact them (or the reasons they wished they would have enacted a particular move).

For the interviews, analyses related to TCs' school enactments focused primarily on responses to the post-interview questions related specifically to School GOP. TCs were more forthcoming with their responses to these questions, which provided valuable insights into the school classrooms where I was not an active participant. For example, TCs shared information 
about the students in their classes that informed my understanding of why they chose to use some teaching moves in certain ways.

The data for each TC varied significantly for their school enactments - each TC displayed different strengths and weaknesses in their planning and in their IA enactments. Based on the interviews and video annotation data, their perceptions of their school enactments also varied greatly. As a result of this variation, rather than identifying overarching themes, I chose to analyze RQ3 through a series of vignettes, one for each TC. Organizing my results to RQ3 in this way provided more insight into the TCs' school contexts. These vignettes, along with my commentary highlighting salient aspects of each TCs' school enactment, may be found in Chapter 4.

In this chapter, I have outlined my methods of data collection and analysis. Video data served as the central data source for my study, but TCs' video annotations and interview responses, along with my own reflective journal, provided valuable insights as I aimed to answer my research questions. In Chapter 4, I highlight themes that have emerged from my investigation into the trends I outlined above, which address my three research questions around the ways TCs developed skill with teaching responsively, the role that coaching during rehearsals played, and the ways in which those skills informed TCs' practice in schools. 


\section{CHAPTER FOUR}

\section{Findings}

As described in Chapters 1 and 2, responsive teaching has been shown to benefit students through a focus on their ideas (rather than the teacher's ideas) and by promoting more equitable classroom instruction. This type of teaching is intellectually demanding but can be learned and developed over time. TEs can support TCs in their development as responsive teachers by focusing methods courses on responsive teaching practices - a subset of core practices that also focuses on responsive teaching. Examples of responsive teaching practices include eliciting and responding to student thinking and orienting students to one another's ideas. Examples of responsive teaching moves - teacher actions at a finer grain size than practices-include making connections among student ideas and asking students to revoice other students' ideas.

Development in responsive teaching is demonstrated in part by TCs' judicious use of these moves (as practical tools).

Research on responsive teaching in teacher education is still in its early stages. Much of the work that has been done in mathematics has focused on elementary TCs' developing enactment of responsive teaching practices, but secondary mathematics TCs need support in enacting responsive teaching practices as well. Therefore, in an effort to contribute to this work, I aimed to answer the following three research questions:

1. How do TCs develop skill in teaching mathematics responsively within a methods course in which cycles of investigation and enactment are a central pedagogy?

2. How does coaching in methods affect TCs' responsive teaching?

3. To what extent do TCs teach responsively in school settings?

In the sections that follow, I discuss my findings in response to the research questions. 


\section{RQ1: How Do TCs Develop Skill in Teaching Mathematics Responsively Within a Methods Course in Which Cycles of Investigation and Enactment Are a Central Pedagogy?}

As TCs progressed through the experiences of multiple cycles of investigation and enactment in the methods course, they demonstrated their developing skill in responsive teaching, in part through their enactments of responsive teaching moves. In some cases, a single move was used in multiple ways. In most cases, each move was used with varying degrees of responsiveness. Even so, my analyses revealed that unresponsive enactments of teaching moves that were used habitually did not necessarily compromise the responsiveness of an entire studentteacher exchange.

In the sections that follow, I first discuss the ways in which TCs developed skill in responsive teaching by attending to student ideas in their planning (through the use of planning materials) and during class discussions (through responsive enactment of teaching moves). I then describe teaching moves that were consistently enacted responsively by TCs. Next, I highlight examples of teaching moves that are positioned as responsive in the literature and were attempted regularly by TCs, but were not always carried out responsively. Finally, I discuss moves that were enacted habitually as evidence that a single unresponsive teaching move does not necessarily compromise the responsiveness of an entire exchange.

Attention to student ideas in class discussion. A key feature of responsive teaching is attending to student ideas, rather than simply preparing lecture notes and presenting information at the front of the room. In planning, responsive teachers should anticipate student reasoning and make deliberate preparations for how and when students will share their ideas during the lesson. In practice, responsive teachers must actually do the work of keeping student ideas at the center of class discussions. TCs' planning materials, along with their enactments of responsive teaching 
moves, provide evidence of their developing skill in responsive teaching. In the sections that follow, I begin by discussing how TCs used the planning materials I provided. Then, I share some descriptive data around the S-T exchanges that occurred during the IA rehearsals in methods.

Planning materials. The protocols and pre-planners are two pedagogical tools that are intended to support TCs as they attend to student thinking by requiring TCs to anticipate student reasoning ahead of time. For example, the Going Over a Problem and Card Sort pre-planners asked TCs to anticipate and record potential correct and incorrect student reasoning. This exercise allowed TCs to start thinking about the problems or prompts from a student's perspective. The TCs in this study used these planning materials differently, some more effectively than others. I first consider the ways in which TCs anticipated student reasoning using the planning materials. I then discuss notes TCs wrote on their planning protocols—notes that provide evidence of the TCs' consideration of responsive teaching prior to rehearsing the IAs.

Anticipating student reasoning. In this section, I highlight planning materials from Bridget and Julian to illustrate the range of abilities displayed by TCs as they anticipated student reasoning. The other two TCs, Charity and Grace, anticipated student reasoning in ways that were more consistent with Bridget's planning.

In her pre-planner for the first IA, GOP, Bridget successfully anticipated multiple examples of correct and incorrect student reasoning (see Figure 9), all of which were fairly procedural in nature (e.g., incorrectly adding integers or performing correct steps, but in different orders). An example of anticipating reasoning from a conceptual viewpoint would have been pointing out that although a student might perform all of the steps correctly to arrive at $0=0$, the 
student might not realize that this means the equation has infinitely many solutions. Bridget's planning work in Figure 9 does not indicate that she considered that possibility. In fact, during the rehearsal, Stephanie ${ }^{3}$ did arrive at the answer $0=0$, but Stephanie interpreted this to mean that the answer was $c=0$. In her video annotation, Bridget commented:

This moment was challenging for me because I did not expect them to have a value for $c$. When I was planning how to teach it, I planned to use an answer similar to this because that is where I ended up at first. I wasn't really sure where to begin starting with [Stephanie's] answer [of $c=0]$.

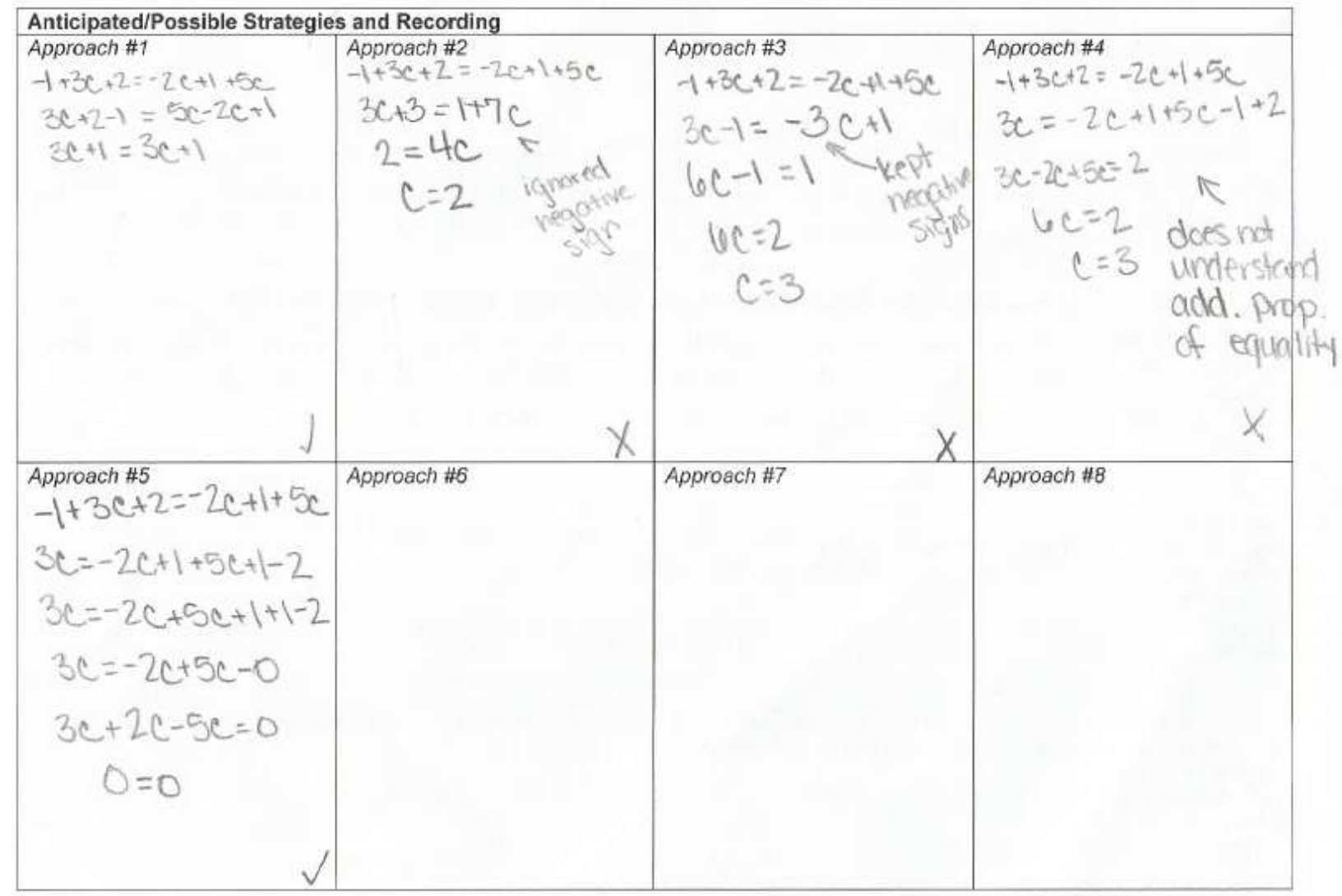

Figure 9. Bridget's Pre-Planner for Going Over a Problem

${ }^{3}$ Stephanie refers to me in the role of student. 
Perhaps as a result of this instance during the first IA, Bridget considered more conceptual approaches to the problem in her pre-planner for the second IA (CthenC). Bridget has provided four possible solutions to the problem (See Figure 10). Note that the pre-planner for this IA did not specifically ask TCs to anticipate both correct and incorrect student reasoning, which may explain why Bridget only provided examples that correctly solved the problem for CthenC. Her thinking is particularly evident in response to the question "How will STUDENTS APPROACH the task? What will they say?" In addition to more traditional approaches (\#1 and \#2), we can see that she also considered several less traditional solutions to the problem. (Two of those approaches are worked out; see approaches \#3 and \#4.) This illustrates Bridget's flexibility in problem solving, as well as her ability to anticipate student reasoning.

It is important to note that it remains unclear exactly what is responsible for Bridget's development of these planning skills. While the experience she gained from the first IA may have helped her prepare for the second IA, it is also likely that the differences in prompts on the planning materials for GOP and CthenC contributed to how she prepared for each IA. Bridget's personal understanding of the mathematics also would have come to bear on her preparation for each IA; had Bridget been assigned different mathematical problems, her planning may have been more or less thorough, depending on her level of understanding of the content. 

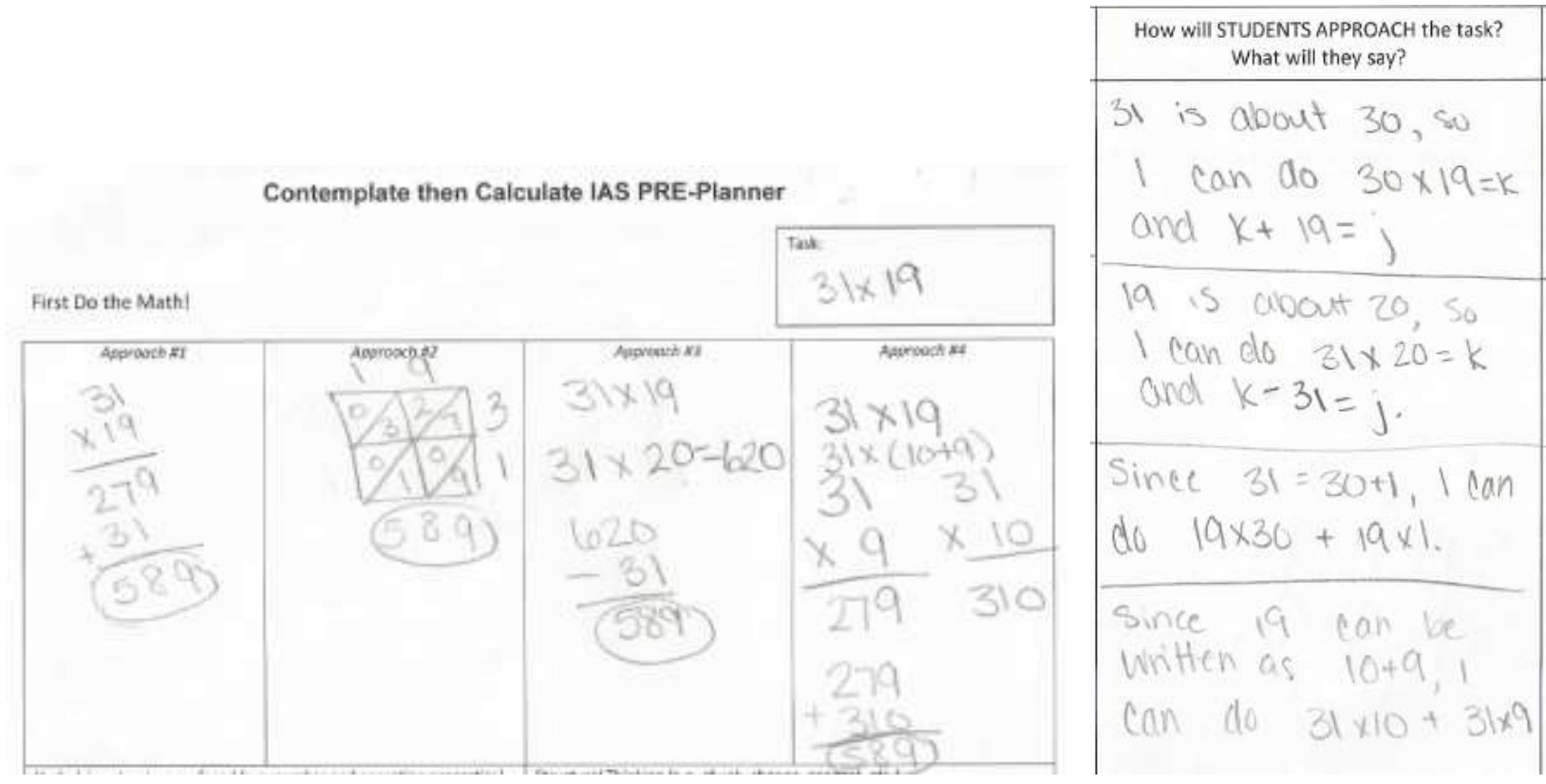

Figure 10. Bridget's Pre-Planner for Contemplate then Calculate

From the beginning of the course, Bridget was able to successfully anticipate student reasoning by identifying multiple strategies (both correct and incorrect) to address her prompts. Julian, on the other hand, initially expressed difficulty in completing these sections of the preplanners. During the mid-interview, when I asked Julian if he had any questions or comments about the rehearsal process, he responded:

I really like the rehearsals and the coaching. The only thing that I'm really having trouble with is those pre-planning papers that you give us...I just feel like it's kind of hard for me, I guess. I've got to get more practice with that.

When I asked him to describe what he found difficult about the planning papers, he said, "Part of it is, thinking about...when you had to write different ways that the students might solve the problem, that was actually pretty hard.” At that point, I emphasized that this was a learning experience and offered to help Julian think of different approaches for the remaining IAs. He did 
not request my help in preparing for the final two IAs, Card Sort and WODB. It is not clear why Julian decided to complete those pre-planners on his own, but it may have been partially a result of the differences in the structure of the final IAs and their planning materials, which prompted TCs in more specific ways than the first two IAs.

The pre-planners for GOP and CthenC provided a general prompt that asked TCs to write different approaches that students might use to solve the problem. The pre-planners for Card Sort and WODB, however, asked TCs to anticipate student reasoning specifically, for each individual card or tile. For example, the pre-planner for the Card Sort IA asked TCs to classify each individual card as an example or non-example, explain whether students are likely to find that card easy or difficult to sort, and then provide a rationale for that decision. These more pointed questions might have provided some additional guidance to Julian, which gave him more confidence to plan the final two IAs on his own. In fact, at the post-interview, he said:

I like how we did the handouts that you gave us and you had to write down your goals and the steps and you gave each step and each sub-step with it, I guess. I really enjoyed those. They helped me a lot.

He went on to explain that the planning materials helped provide "a broader understanding of things we can do in a math class that would break up using direct instruction all the time."

Preparing for enactment. As TCs turned their attention to preparing for the enactment of an IA, the structure of the protocols continued to support their efforts to maintain a focus on student thinking by suggesting questions to be asked and actions that could be taken. For example, the protocol for WODB directs TCs to ask a student which tile they thought did not belong and why. In each of the IAs, the TC is responsible for recording relevant student ideas to make them visible for the entire class. (Part of the design of the rehearsal process involves the 
TC recording student ideas, as opposed to bringing students to the board. This is partially highlighted by the structure of the IA protocols.) As they prepared for their rehearsals, TCs were mindful of the importance of their recording, as evidenced by written comments such as, "write exactly what students say" on their protocols (see Figures 11 and 12.) This is interesting because although the protocols do direct TCs to record student ideas on the board, they are less specific about the care with which TCs record those ideas; TCs who wrote these notes extended that instruction. This may have partially been a result of a compliment I gave Grace during the very first IA rehearsal. I praised Grace for "doing a really good job of writing only what [Charity] is saying." My highlighting of that move early in the semester may have caused TCs to be especially attentive to their recording. Some TCs' awareness of the importance of recording seemed to increase over time. Charity, for example, only made notes about recording during the final two IAs (Figure 12), which suggests that she gave more attention to her recording as the semester progressed.

\begin{tabular}{|c|c|}
\hline $\begin{array}{l}\text { prompt a student to summarize the arguments made } \\
\text { that support this being an example of } \\
\text { Elicit arguments for why it might be a non- }\end{array}$ & $\begin{array}{l}\text { - Wrate exactly what } \\
\text { Whestodigy says, }\end{array}$ \\
\hline
\end{tabular}

Figure 11. Julian's notes about careful recording - Card Sort IA

\section{[Record student reasoning throughout]}

\begin{tabular}{l|l|}
$\begin{array}{l}\text { Record relevant ideas on or alongside the panel. } \\
\text { This might include terminology that is used, as well } \\
\text { as notations/markings on the panel. }\end{array}$ & $\begin{array}{l}\text { students saty } \\
\text { students what }\end{array}$ \\
\hline
\end{tabular}

Figure 12. Charity's notes about careful recording - Card Sort IA (top), WODB IA (bottom) 
The protocols also ask TCs to provide time for students to think about a problem or prompt. TCs wrote frequent reminders to themselves about this aspect of the IAs as well, with notes such as, "Think-Pair-Share” and "Give think time.” (See Figure 13.) Although the protocols directed TCs to give students time to think about problems and other students' solutions, their written reminders demonstrate their understanding of the importance of allowing students time to make sense of the mathematics being discussed. During their initial Teaching Demos, none of the TCs provided time for students to think about or solve the problem on their own. Therefore, this attention to giving students time to think on their own provides evidence of their development as responsive teachers.
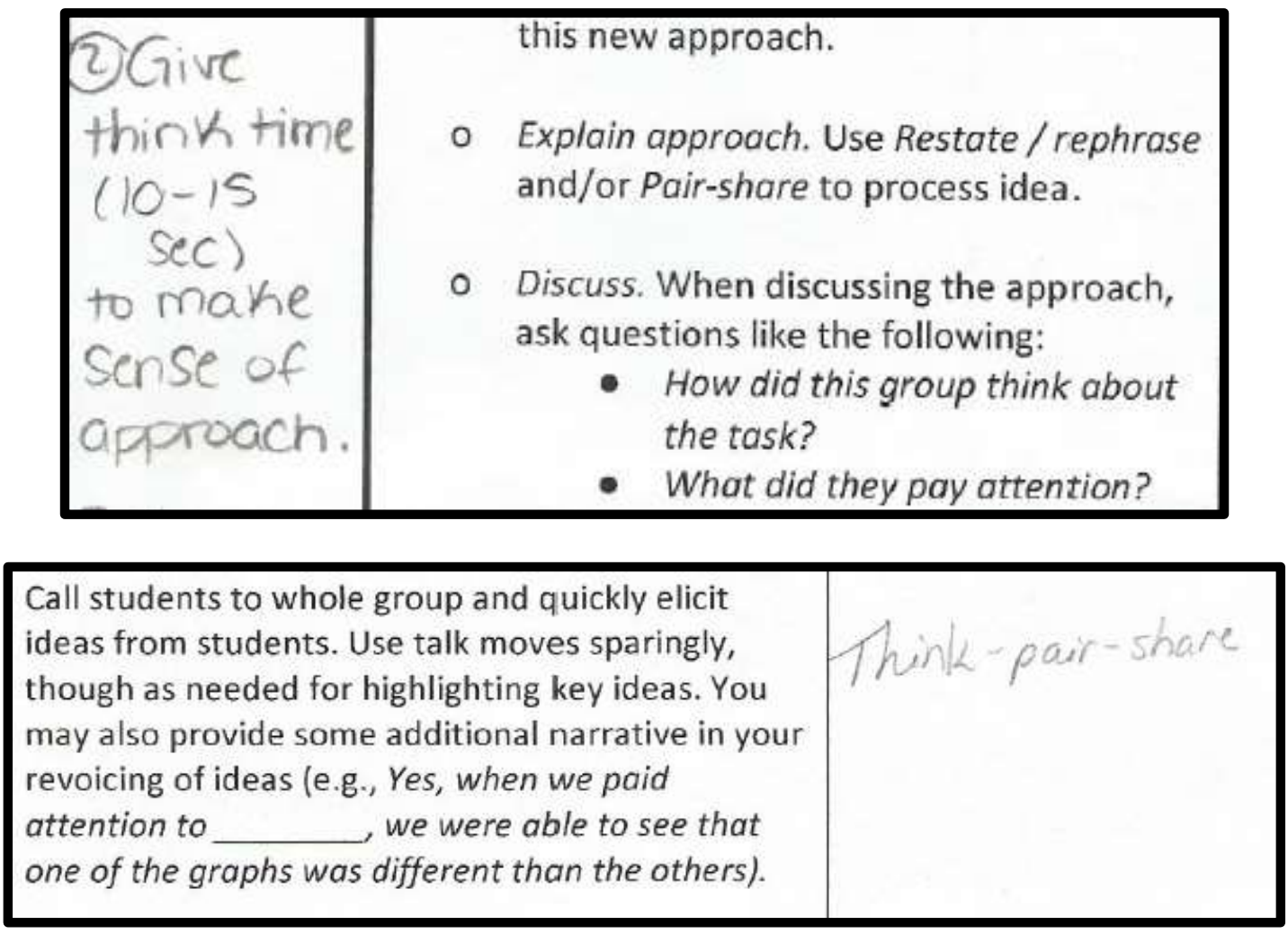

Figure 13. Planning related to T Prompts S to Take Think Time (Grace, CthenC; Charity, WODB) 
TCs used the planning materials differently over time. Grace wrote extensive notes on her protocols for the first two IAs. In her video annotation for CthenC, Grace noted, "I felt supported in my teaching because this method was one that I had prepared for and documented in my planner." For the final two IAs, however, Grace wrote very few notes on her protocols. It is not clear why there was such a shift in her planning notes, but unsurprisingly, comments about the helpfulness of the planning materials did not appear in her video annotations for the final two IAs.

In contrast, Charity's planning notes consistently showed that she had put significant effort into planning for each rehearsal, but the kinds of notes she wrote on her protocols changed over time. For the first two IAs, she wrote specific questions to ask during the rehearsal, but little about responsive teaching moves. For the last two IAs, her notes focused less on specific questions to be asked and more on responsiveness, with reminders to "write exactly what students say" (Figure 12) and "Think-Pair-Share" (Figure 13). This shift in Charity's planning notes suggests that she developed greater attentiveness toward responsive teaching as the semester progressed.

Planning is an important first step in developing skill as a responsive teacher. Anticipating student reasoning ahead of time helped TCs to feel more comfortable facilitating a class discussion around that reasoning. As they prepared to rehearse the IAs, TCs noted on their protocols responsive teaching moves that could be enacted at specific points. In the end, however, what matters most is how TCs used their planning materials in their enactments of responsive teaching. In the next section, I provide descriptive data, which provides an overview of TCs' responsive teaching throughout the semester. 
Descriptive data. Recall that S-T instances are exchanges between the teacher and one or more students that focus on a single idea. Descriptive data on S-T instances during IA rehearsals shows that TCs engaged with student ideas more frequently and spent longer periods of time engaging with those ideas during the IAs than during the Teaching Demos (see Table 10). There were nearly twice as many S-T instances during each of the IAs when compared with the Teaching Demos. Additionally, each S-T instance lasted approximately three times as long (or more) during the IAs than during the Teaching Demos. This suggests that the TCs were leading the IAs in ways that engaged student ideas more frequently and more deeply than in the Teaching Demos. Figure 14 contains representative examples of short and long S-T exchanges. Table 10

Number and Length of S-T Instances per IA

\begin{tabular}{ccccc}
\hline Demo/IA & $\begin{array}{c}\text { Average Length } \\
\text { of Demo/IA }\end{array}$ & $\begin{array}{c}\text { Total Number } \\
\text { of S-T } \\
\text { instances }\end{array}$ & $\begin{array}{c}\text { Average } \\
\text { Number of S-T } \\
\text { instances per } \\
\text { Demo/IA }\end{array}$ & $\begin{array}{c}\text { Average length } \\
\text { of S-T instances } \\
\text { per Demo/IA }\end{array}$ \\
\hline Teaching Demo & 4.5 min. & 27 & 6.75 & $13 \mathrm{sec}$. \\
GOP & $16.7 \mathrm{~min}$. & 53 & 13.25 & $37 \mathrm{sec}$. \\
CthenC & $17.0 \mathrm{~min}$. & 53 & 13.25 & $43 \mathrm{sec}$. \\
Card Sort & $29.2 \mathrm{~min}$. & 64 & 16 & $55 \mathrm{sec}$. \\
WODB & $23.8 \mathrm{~min}$. & 50 & 12.5 & $1 \mathrm{~min} .10 \mathrm{sec}$.
\end{tabular}

${ }^{a}$ Calculated using the Instance Frequency Report from Studiocode 


\begin{tabular}{|c|c|}
\hline Julian (Teaching Demo) & Julian (WODB) \\
\hline $\begin{array}{l}\mathrm{T}^{4} \text { : Can somebody tell me what the } \\
\text { mean is? }\end{array}$ & $\begin{array}{l}\text { T: Stephanie, I heard you picked a different card. } \\
\text { Which one did you pick and why did you pick it? }\end{array}$ \\
\hline Bridget: It's the average. & Stephanie: I picked the third one, $-2 x+4$. \\
\hline T: Yes. & T: And why did you pick it? \\
\hline \multirow{7}{*}{$\begin{array}{l}\text { Julian proceeded to calculate the mean } \\
\text { himself at the board. }\end{array}$} & Stephanie: Because it has a negative slope. \\
\hline & [T records Stephanie's response on the board.] \\
\hline & T: How did you know that it had a negative slope? \\
\hline & $\begin{array}{l}\text { Stephanie: Because the number in front of } x \text {, the } \\
\text { coefficient of } x \text {, is negative and that represents the } \\
\text { slope. And it's the only one that's negative. }\end{array}$ \\
\hline & T: Okay. Were there any other reasons for this one? \\
\hline & Stephanie: (shakes head) \\
\hline & T: No? Okay. \\
\hline
\end{tabular}

Figure 14. Sample S-T Instances from Teaching Demo and WODB

Notice that the S-T instance from Julian's Teaching Demo is very short. His question required a response of only a few words from Bridget, without a focus on her understanding of "average" or the way to calculate it. In contrast, the S-T instance from Julian's WODB rehearsal is longer, and he provided more opportunities for me (Stephanie) to share my reasoning about the tile I had chosen, making this instance far more responsive.

\footnotetext{
${ }^{4} \mathrm{~T}$ represents the rehearsing TC in each transcript.
} 
As TCs developed skill in teaching mathematics responsively, three teaching moves emerged as ones that TCs tended to enact responsively: T Revoices, Ask Different $S$ to Revoice, and T Makes Connection. I discuss TCs' enactments of each of these three moves in detail in the sections that follow. The next section begins with a general discussion of revoicing moves, followed by a more specific discussion of each individual revoicing move, and then a comparison of the enactments of those two moves. Lastly, in the final subsection related to attending to student ideas in class discussions, I discuss TCs' enactments of the move T Makes

\section{Connection.}

Responsive teaching moves enacted responsively. TCs enacted a variety of teaching moves aimed at keeping student ideas central to class discussion, but two revoicing moves placed explicit emphasis on student thinking. Revoicing an idea indicates that someone else (the teacher or another student) restates an idea in their own words, but without adding any information. Revoicing might involve emphasizing a specific part of a student's idea as a means of working toward the mathematical goal. Revoicing moves are worth highlighting because of their tendency toward responsiveness. When the idea being revoiced originates with a student, this move has the potential to be responsive by keeping the student's idea at the center of the class discussion. TCs enacted two different types of revoicing moves during the semester: $T$ Revoices and Ask Different S to Revoice.

T Revoices. Sometimes, a TC chose to personally revoice a student idea. TCs in this study used this move fairly consistently over the course of the semester, which indicates one aspect of TCs' attention to responsiveness and one way in which this revoicing move supported responsive teaching. Table 11 displays the number of responsive and unresponsive instances of $T$ Revoices organized by IA and TC. 
Table 11

Responsive and Unresponsive Instances of T Revoices

\begin{tabular}{|c|c|c|c|c|c|c|c|c|c|c|}
\hline \multirow[b]{2}{*}{ Demo/IA } & \multicolumn{2}{|c|}{ Julian } & \multicolumn{2}{|c|}{ Bridget } & \multicolumn{2}{|c|}{ Charity } & \multicolumn{2}{|c|}{ Grace } & \multicolumn{2}{|c|}{ Totals } \\
\hline & Resp. & Unresp. & Resp. & Unresp. & Resp. & Unresp. & Resp. & Unresp. & Resp. & Unresp. \\
\hline Teaching Demo & -- & -- & -- & -- & -- & 1 & -- & -- & 0 & 1 \\
\hline GOP & 1 & 2 & 2 & -- & -- & -- & 1 & -- & 4 & 2 \\
\hline CthenC & 1 & 1 & 1 & -- & -- & -- & 2 & -- & 4 & 1 \\
\hline Card Sort & -- & -- & 1 & -- & -- & -- & 1 & -- & 2 & 0 \\
\hline WODB & 3 & 1 & 2 & -- & 1 & -- & 3 & -- & 9 & 1 \\
\hline Totals & 5 & 3 & 6 & 0 & 1 & 1 & 7 & 0 & 19 & 5 \\
\hline
\end{tabular}


T Revoices was considered responsive for 19 of the 24 instances that occurred in methods. Responsive instances kept student reasoning central in some way, perhaps by ensuring proper recording of a student's idea or more concisely stating a student's idea to make it more accessible to the rest of the class. In eight of the 19 responsive instances of T Revoices, the move was used to highlight or emphasize important mathematical ideas in a student's response. Consider the following excerpt from Bridget's CthenC rehearsal. Earlier in the IA, Grace had estimated that $31 \times 19$ is 600 , rather than solving for an exact answer.

T: $\quad$ Grace, you had a different answer. Can you walk me through what you did?

Grace: Yeah, so I saw that the 31 was close to 30, so I changed it to 30. And that the 19 is close to 20 , so I changed it to 20 . Then I went ahead and just multiplied those two numbers to get an approximate answer.

$\mathrm{T}: \quad$...So are we saying 600 is an answer to our problem then, or about an answer? Grace: $\quad 600$ is an approximate answer.

T: $\quad$ So then, that's saying $31 \times 19$ is not 600 , but it's close. Is that what you're saying?

Grace: Yeah.

Notice that when Bridget revoiced Grace's idea, she validated Grace's reasoning while still making a clear point that the correct answer to $31 \times 19$ is not 600 . This was considered responsive because Bridget maintained a focus on Grace's idea. It is also worth noting that, while keeping Grace's idea central, Bridget's use of this revoicing move also slightly shifted the focus of the discussion from Grace's solution process to the fact that the answer was approximate, not exact. 
T Revoices was considered unresponsive for five of the 24 instances that occurred in methods. Note that four of these five unresponsive instances occurred early in the semester, during the Teaching Demos and the first two IAs (GOP and CthenC). In these instances, TCs used the move as a way to bring out a specific idea or to highlight some related idea that was not specifically addressed by the student. These purposes shifted the focus from the student's idea to the teacher's idea, making these enactments unresponsive. Figure 15 provides two examples of this move being used unresponsively.

\begin{tabular}{|l|l|}
\hline Unresponsive (Julian, GOP) & Unresponsive (Julian, WODB) \\
\hline $\begin{array}{l}\text { T: ... show me the steps that you took to solve } \\
\text { this. }\end{array}$ & $\begin{array}{l}\text { T: What was one detail that you had? } \\
\text { Grace: We should pay attention to the y- } \\
\text { intercept. }\end{array}$ \\
$\begin{array}{l}\text { T: Okay, so you multiplied this first, and } \\
\text { then... }\end{array}$ & $\begin{array}{l}\text { T: Okay. Why do you think we need to pay } \\
\text { attention to the y-intercept? }\end{array}$ \\
$\begin{array}{l}\text { Julian continued to write the solution without } \\
\text { input from Grace. }\end{array}$ & $\begin{array}{l}\text { Grace: Because it's important to know where } \\
\text { it crosses the y-axis. } \\
\text { T: Okay. So we need to know where on this y- } \\
\text { axis that it starts at. Is that what you're trying } \\
\text { to say? }\end{array}$ \\
\hline
\end{tabular}

Figure 15. Unresponsive enactments of T Revoices

During his GOP rehearsal, Julian's enactment of $T$ Revoices led him to take control of the class discussion by solving the problem without further student input. He only accepted a brief comment from Grace to start, but then began to solve the equation himself. Because the focus of the class discussion was shifted back to the teacher's thinking, this instance was considered unresponsive.

Julian's enactment of T Revoices during his WODB rehearsal, while still unresponsive, had a different result than his previous enactment of the move. He did not shift the discussion to 
his own ideas, but here his revoicing did not seem to serve any purpose. Grace's statement was already concise and mathematically correct, which left no real need for Julian to revoice her idea. Thus, he may have used the revoicing move more out of habit. While habitual uses of the move are not necessarily detrimental to the IA, they do not have student reasoning as their focus, which renders them unresponsive.

Over the course of the semester, responsive instances of $T$ Revoices remained fairly consistent, while unresponsive instances of the move slightly decreased (see Table 11). This is indicative of one aspect of TCs' development of skill in responsive teaching. Another aspect of TCs' development involved a different revoicing move: Ask Different $S$ to Revoice.

Ask Different S to Revoice. The second type of revoicing move enacted by TCs was Ask Different $S$ to Revoice. Rather than revoicing the idea themselves, TCs used this move to ask a student to revoice the original student's idea. This is one move teachers can use to achieve the responsive teaching practice of orienting students to each other's ideas. Ask Different $S$ to Revoice has the potential to be responsive because it allows the teacher to maintain a focus on the first student's idea, while also allowing the students (rather than the teacher) to drive the discussion.

This teaching move seemed to be new for the TCs in this study—none asked a different student to revoice during their Teaching Demos. After being introduced to the move, however, they made plans to enact it throughout the semester, as evidenced in their planning protocol notes (see Figure 16). 


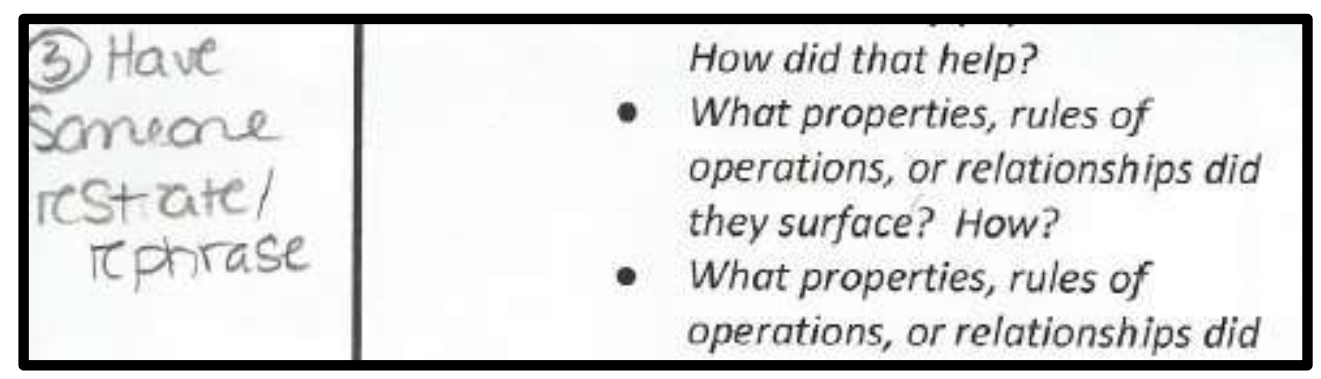

Teacher calls on student (this can be strategic based on the monitoring). Student shares card.

[Record the card in the example column on the board]

Figure 16. Reminders to Ask Different S to Revoice (Grace, CthenC; Charity, Card Sort)

Over the course of the semester, TCs enacted Ask Different $S$ to Revoice 22 times in methods. Of those instances, 20 were responsive, with four of the responsive instances being a direct result of coaching (see Table 12). The two unresponsive instances were labeled as such because the teacher asked a student (1) to revoice instructions that had been stated unclearly or (2) to revoice only after a student indicated they had nothing new to contribute to the discussion. In both of these instances, student reasoning was not the focus of the exchange. Figure 17 provides examples from the IAs in methods which illustrate the use of this move in both responsive and unresponsive ways. 
Table 12

Responsive and Unresponsive Instances of Ask Different S to Revoice

\begin{tabular}{|c|c|c|c|c|c|c|c|c|c|c|}
\hline Demo/IA & \multicolumn{2}{|c|}{ Julian } & \multicolumn{2}{|c|}{ Bridget } & \multicolumn{2}{|c|}{ Charity } & \multicolumn{2}{|c|}{ Grace } & \multicolumn{2}{|c|}{ Totals } \\
\hline GOP & -- & -- & 2 & -- & $1(1)$ & -- & 1 & -- & 5 & 0 \\
\hline CthenC & $1(1)$ & -- & 1 & -- & $1(1)$ & -- & 2 & -- & 7 & 0 \\
\hline WODB & (2) & 1 & -- & 1 & 2 & -- & $2(1)$ & -- & 7 & 2 \\
\hline Totals & 4 & 1 & 3 & 1 & 7 & 0 & 6 & 0 & 20 & 2 \\
\hline
\end{tabular}

Note. Instances prompted by coach are shown in parentheses. For example, 1(1) indicates that two responsive instances of Ask Different $S$ to Revoice took place, with one of those instances being prompted by the coach. 


\begin{tabular}{|c|c|}
\hline Responsive (Julian, CthenC) & Unresponsive (Bridget, WODB) \\
\hline 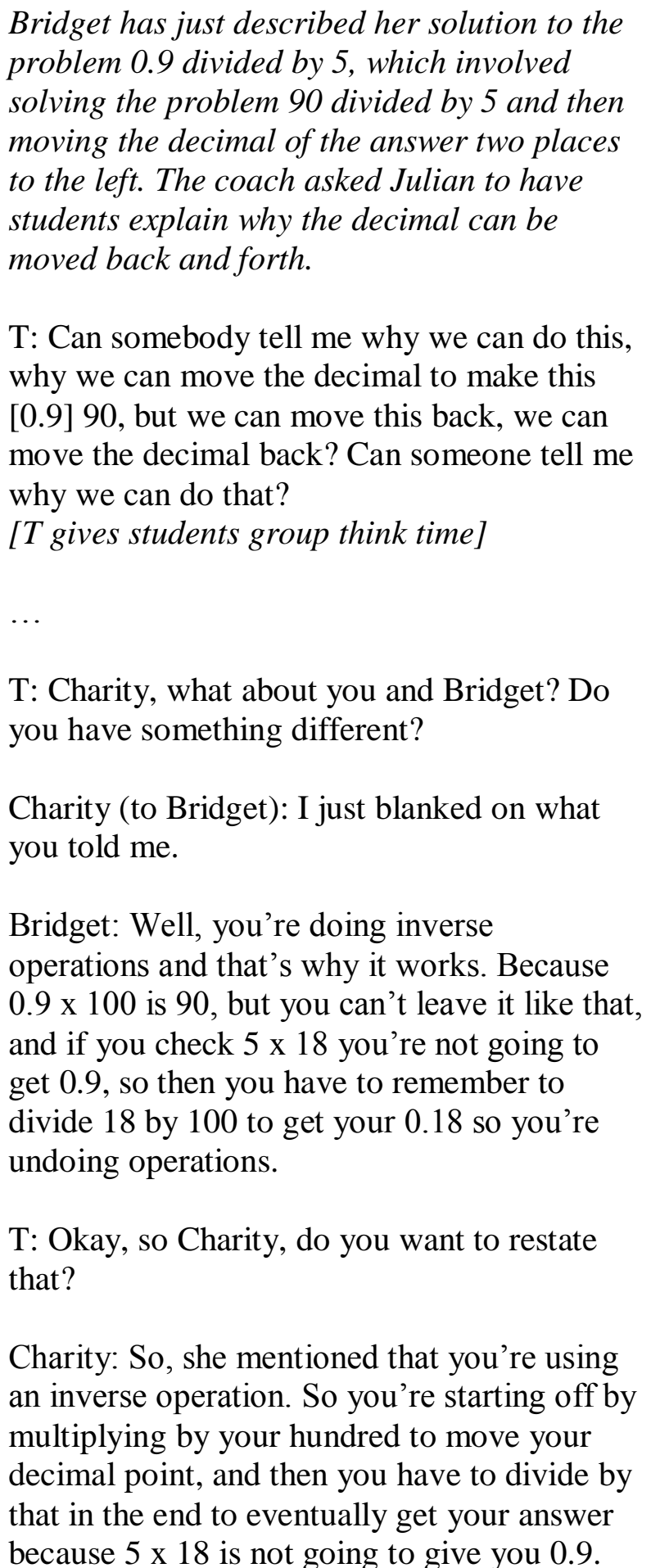 & $\begin{array}{l}\text { The teacher is eliciting reasons why the third } \\
\text { tile could be the one that doesn't belong. The } \\
\text { teacher has already elicited at least one } \\
\text { reason from each of the three other students. } \\
\text { T: Julian, is there anything else? } \\
\text { Julian: No, nothing else. } \\
\text { T: All right, can you kind of restate why this } \\
\text { graph is different? } \\
\text { Julian: Restate it? } \\
\text { T: Mm-hmm. } \\
\text { Julian: [reading from the board] Well, Grace } \\
\text { talked about too, that it starts high on the left } \\
\text { and ends low on the right, and there's also a } \\
\text { minimum value of - } 4 \text {, and the maximum rests } \\
\text { on the } x \text {-axis at, I think it's } 3,3 \text { or } 4 \text {. } \\
\text { T: That one is kind of hard to tell if it's } 3 \text { or } 4 \text {, } \\
\text { but it's close to one of them. }\end{array}$ \\
\hline
\end{tabular}

Figure 17. Responsive and Unresponsive Instances of Ask Different S to Revoice 
Notice that Julian asked Charity to revoice what Bridget said, which served to keep Bridget's idea at the center of discussion and to ensure that Charity understood it, making the move responsive. In contrast, Bridget asked Julian to revoice only after he said he had nothing new to contribute. This revoicing amounted to Julian reading the list of characteristics from the board without any further questioning from Bridget. This move neither clarified any of the ideas on the board, nor did it seem to clarify Julian's thinking, making it unresponsive. What is evident in these examples of Ask $S$ to Revoice is that, in order to develop skill in responsive teaching, TCs need to be supported not only in enacting the move, but also in considering when to enact the move to maintain a focus on student reasoning.

Comparing T Revoices and Ask Different S to Revoice. A comparison of the enactments of T Revoices and Ask Different $S$ to Revoice is useful because although both involve revoicing, they make different demands on the TC. T Revoices is a fairly ubiquitous move in teaching and as such, the TCs easily enacted this move on their own. Revoicing an idea personally also allows the TC to maintain more control of the discussion, which is likely to provide a more direct path toward achieving the mathematical goal of the IA.

In contrast, Ask Different $S$ to Revoice was a new move for TCs. As a result, TCs had to be more deliberate in their planning and enactments of this revoicing move. When asking a student to revoice, the TC allows students to take on more responsibility in directing the class discussion. As a result, it could take longer or be more difficult for the TC to achieve the mathematical goal of the IA. Thus, enactment of Ask Different S to Revoice presents more of a challenge for TCs, both in deciding when to enact the move and then deciding how to guide the discussion following its enactment. Table 13 provides a comparison of the number of times each TC responsively enacted the $T$ Revoices move and the Ask $S$ to Revoice move. 
Table 13

Comparison of responsive enactments of T Revoice and Ask S to Revoice

\begin{tabular}{|c|c|c|c|c|c|c|c|c|c|c|}
\hline \multirow[b]{2}{*}{ Demo/IA } & \multicolumn{2}{|c|}{ Julian } & \multicolumn{2}{|c|}{ Bridget } & \multicolumn{2}{|c|}{ Charity } & \multicolumn{2}{|c|}{ Grace } & \multicolumn{2}{|c|}{ Totals } \\
\hline & $\begin{array}{c}\mathrm{T} \\
\text { Revoice }\end{array}$ & $\begin{array}{c}\text { Ask S } \\
\text { to } \\
\text { Revoice }\end{array}$ & $\begin{array}{c}\mathrm{T} \\
\text { Revoice }\end{array}$ & $\begin{array}{c}\text { Ask S } \\
\text { to } \\
\text { Revoice }\end{array}$ & $\begin{array}{c}\mathrm{T} \\
\text { Revoice }\end{array}$ & $\begin{array}{c}\text { Ask S } \\
\text { to } \\
\text { Revoice }\end{array}$ & $\begin{array}{c}\mathrm{T} \\
\text { Revoice }\end{array}$ & $\begin{array}{c}\text { Ask S } \\
\text { to } \\
\text { Revoice }\end{array}$ & $\begin{array}{c}\mathrm{T} \\
\text { Revoice }\end{array}$ & $\begin{array}{c}\text { Ask S } \\
\text { to } \\
\text { Revoice }\end{array}$ \\
\hline Teaching Demo & -- & -- & -- & -- & -- & -- & -- & -- & 0 & 0 \\
\hline GOP & 1 & -- & 2 & 2 & -- & 2 & 1 & 1 & 4 & 5 \\
\hline CthenC & 1 & 2 & 1 & 1 & -- & 2 & 2 & 2 & 4 & 7 \\
\hline Card Sort & -- & -- & 1 & -- & -- & 1 & 1 & -- & 2 & 1 \\
\hline WODB & 3 & 2 & 2 & -- & 1 & 2 & 3 & 3 & 9 & 7 \\
\hline Totals & 5 & 4 & 6 & 3 & 1 & 7 & 7 & 6 & 19 & 20 \\
\hline
\end{tabular}


Comparisons of these two moves varied among TCs; some used both moves fairly equally, while others tended to use one move more than the other. Looking across the IAs in methods, TCs did not necessarily use either move more over time. However, this is not necessarily indicative of a lack of TC development. An important part of responsive teaching is using moves judiciously. Thus, overuse of the move could be just as detrimental as not using it at all. TCs' comments on planning materials and in their video annotations provide evidence that they were considering when and how to enact these moves appropriately, even if the result was not always what they planned.

The totals in Table 13 show that Charity used the move Ask Different $S$ to Revoice far more often than $T$ Revoices. Charity's video annotations and planning materials reveal that she was mindful of asking students to revoice throughout the semester. For example, in her video annotation for CthenC she wrote, "The final part of this clip shows student reasoning ... by having Julian explain what Grace had done. However, in hindsight I probably should have had Bridget restate because Julian had solved the problem using Grace's method." This comment indicates her consideration of not just using the move, but how to best enact the move. As the semester progressed, Charity continued to be mindful of asking students to revoice. In the planning materials for both the Card Sort and WODB, Charity wrote multiple times, "Have another student restate?" as personal reminders to enact the move during her rehearsals (see Figure 14).

Grace also provided evidence that she was mindful of asking students to revoice. In her planning materials for $\mathrm{CthenC}$, she wrote a reminder to "have someone restate/rephrase approach" (see Figure 14). Then, in her video annotation following her CthenC rehearsal, she wrote, "Asking another student to restate the process that was taken gave a different student the 
opportunity to share her own reasoning with the class." (While this enactment of Ask Different $S$ to Revoice was labeled as responsive, the imprecision in Grace's comments reveal an incomplete understanding of the purpose of the move. Although she wrote that using the move allowed another student the chance to "share her own reasoning with the class," that is not truly what occurred. In this instance, the second student revoiced the solution strategy of the first studentno new reasoning was shared.) Grace again attended to student revoicing during her WODB video annotation. She wrote, "The students were asked to restate because I needed to determine other students' understanding." These comments provide evidence that Grace was developing skill in deciding when to enact the move for a particular purpose.

In addition to her attention to asking another student to revoice, Grace also gave sustained attention to revoicing student ideas herself. She enacted the move $T$ Revoices more than any other TC during methods. None of the notes on her planning protocols were reminders for her to revoice, which suggests that she had a natural tendency to enact $T$ Revoices. Comparisons of Grace's enactments of $T$ Revoices with her video annotation comments provide evidence that Grace used the move either as a means of working toward the mathematical goal or as a means of coping with a challenging moment. Consider the following excerpt from Grace's Card Sort rehearsal:

T: What was something that was really easy for you to sort?

Julian: $\quad$ Ours was $f(x)=2(x-7)^{2}$.

T: $\quad$ Why was this so easy for you to sort?

Julian: Ours was kind of the same reasoning as that first one, because we have the $(x-7)^{2}$, you have to do that twice and then FOIL it and then you end up getting different $x$ 's with different degrees. 
T: Okay, so after you change the function given here? [T records]... [talking to herself] I'm trying to think of how to say this...[T records]...So you get the same thing as up here, more than one $x$ and the $x$ 's have different degrees?

Julian: Yes.

Grace identified this instance as a challenging moment:

I had a difficult time determining exactly what to write. I can't remember exactly what I wrote, but I knew I needed to condense what was said a little bit and I knew I wanted to write it more generally. The cards that had polynomials written in a way that made it hard to determine if it was actually a polynomial without simplifying were a challenge for me. I wanted to be sure the students knew exactly what the function would look like when rearranged.

However, she also identified this moment as one that moved her toward her mathematical goal for the IA:

Getting the student's thoughts and ideas on the board was the next step toward the goal because the students could continue to formulate their ideas of what makes a polynomial function by hearing [the] other group's thoughts. Here the students gave examples of cards they thought were polynomials.

It seems as though Grace's attention to Ask Different $S$ to Revoice in her planning, coupled with her own natural tendency to revoice, which helped her to overcome challenging moments and/or highlight her goals for the IAs, led her to use the two moves fairly equally.

In contrast to Grace's consistent use of both revoicing moves, Bridget responsively revoiced ideas herself (T Revoices) twice as often as she responsively asked students to revoice each other's ideas (Ask Different $S$ to Revoice). In general, she wrote minimal notes on her 
planning protocols. The only time she wrote about the revoicing moves in planning occurred on her pre-planner for CthenC. In response to the question, How will you RECORD/REPRESENT student approaches? Bridget wrote, "I will restate when recording," and "I will have students rephrase strategies." Similar notes were not present on her protocol for this IA or on any of her planning materials for the other IAs. Bridget's higher number of enactments of T Revoices suggests that, like Grace, this move came naturally to her. However, unlike Grace and Charity, Bridget did not write many notes on her planning materials about asking students to revoice, which may have led her to enact Ask Different S to Revoice less often.

While both $T$ Revoices and Ask Different $S$ to Revoice have a tendency to promote responsive teaching, it is important for TCs to move beyond revoicing student ideas themselves to inviting students to revoice one another's ideas. Such a shift would place more emphasis on the students and their understanding of each other's ideas. The data provides evidence to suggest that deliberate attention is required, in both planning and enactment, to support TCs in making this step.

My attention to student revoicing. The increased attention from Charity and Grace toward asking a different student to revoice may be partially explained by the attention I gave to the move as the teacher educator. My reflective journal provides evidence that I reflected on the move Ask Different S to Revoice frequently throughout the semester. For example, on August 30, after modeling the first IA for the TCs, I wrote, "I wish I had found a way to work in the restate move more," and anticipated that the TCs would need additional guidance in using this move. During the following class, I wrote about a more pointed class discussion focused on this move, which made me feel more confident about the TCs' ability to enact the move when they were 
rehearsing. Later (Sept. 27), I was still lamenting missed opportunities to develop their skills in using this move:

I do wish I would have asked [Grace] to have someone restate why we could use the distributive property on her problem. I'm sure she saw it on my paper, which is why she asked about it, and that's fine, but playing the dual role of coach and student, I lost track of that important point.

During the Card Sort rehearsals, TCs enacted Ask Different $S$ to Revoice only once on their own. I did not interject about this move during these rehearsals, though I am not sure why. I did, however, notice that three of the TCs did not even attempt to enact the move, which led me to share some video clips of the rehearsals with the TCs during our whole-class reflection of the Card Sort IA on Oct. 27. We first viewed a clip from Charity’s Card Sort that showed her responsive enactment of Ask Different $S$ to Revoice. We then viewed clips from each of the other TCs' rehearsals that provided examples of moments when asking a different student to revoice might have contributed positively to the responsiveness of the IA. One such example occurred during Grace's Card Sort, when she was eliciting ideas from Charity:

T: $\quad$ Bridget and Charity, will you give me one that was easily sorted?

Charity: $f(x)=x$.

T: $\quad$ Okay. Tell me a little bit of your reasoning.

Charity: There was only one variable and there's no differing exponents.

T: $\quad$ [records] And you said no differing exponents? What do you mean by that?

Charity: So, with our other one, our reasoning was, say with the $5 x^{4}+5 x^{2}$, there's two $x$ 's, two different exponents. In this one, you only have one variable, no exponent. 
T: So basically the same thing as over there.

Notice that Grace did not fully revoice the idea herself; instead she identified Charity's current reasoning within statements that had already been made. When I asked her if she could remember why she chose to follow up on Charity's reasoning that way, she said, "I think I knew what she meant, but I just wanted to make sure everyone else did, because what she was saying was just, it could have been taken in different ways, I guess." We eventually discussed that asking a student to revoice another student's idea is a different way to ensure that students understand what has been said. We also discussed what to do if a student was unable to revoice another student's idea:

I decided to focus today's reflection on opportunities to engage students with others' ideas. The TCs missed several opportunities to do this during their [Card Sort] rehearsals, so it seemed like something worthy of whole group discussion. In particular, we talked about what a teacher could do if the selected student was unable to restate what another student said, which led to talking (again) about how there are multiple paths to take at any given moment of teaching and how to look for 'signs' to decide on a path.

This class discussion likely resulted in increased mindfulness, for both me and the TCs, about asking students to revoice during WODB, the final IA rehearsed in methods. As I introduced the final IA (WODB), I acknowledged that although we had been discussing the move frequently, my enactments of the move (as the TE) were imperfect, which may have limited TCs' abilities to use the move. On October 30, I wrote:

...I realized how much the TCs are mirroring me when they do their rehearsals, which isn't necessarily a good thing, because I'm not as good as I'd like to be at using talk moves like having students restate other students' ideas. As I came to that realization, I 
tried to incorporate the move a bit more, but it wasn't great. (Then again, none of their ideas came out unclearly or imprecisely enough for me to want to have another student restate them. I guess...that there should be something 'special' about what a student offers to compel me to have students restate.)

The WODB rehearsals saw a slight increase in my coaching interjections around Ask Different $S$ to Revoice, which resulted in TCs' increased enactments of the move. I will discuss this finding further in response to RQ2.

Revoicing student ideas, whether done by the teacher or another student, is one way the TCs in this study developed skill around the responsive teaching practice of eliciting and responding to student thinking. Both $T$ Revoices and Ask Different $S$ to Revoice serve as ways to elicit student ideas publicly to be taken up by the whole class. Once student ideas have been elicited, TCs must decide how to leverage them to work toward their mathematical goals. In the next section, I discuss one teaching move that TCs enacted responsively to make use of student ideas and to move students toward an understanding of the goal for the IA: T Makes Connection.

T Makes Connection. A third responsive teaching move TCs enacted in consistently responsive ways is T Makes Connection. This move involves TCs making connections among students' ideas or between a student's idea and an established mathematical fact. TCs cannot make connections using student ideas until those ideas have been elicited, perhaps partly through the use of the revoicing moves discussed in the previous sections. In addition, there are fewer opportunities for TCs to make connections among students' ideas- the elicitation of multiple student ideas around a single problem does not necessarily mean that making a connection between those ideas will be productive. Finally, making such connections arguably requires more skill than other responsive teaching moves because it calls upon TCs to draw an in-the-moment 
comparison between ideas and then decide how to articulate relevant connections for the class. As a result, this move was enacted less often than either $T$ Revoices or Ask Different $S$ to Revoice. However, when TCs enacted T Makes Connection, the move was used responsively. Table 14 displays the number of enactments of T Makes Connection, organized by IA and TC. Table 14

Instances of T Makes Connection, organized by IA and TC

\begin{tabular}{cccccc}
\hline & Julian & Bridget & Charity & Grace & Totals \\
\hline GOP & -- & 1 & -- & -- & 1 \\
CthenC & -- & -- & -- & -- & 0 \\
Card Sort & -- & -- & -- & 1 & 1 \\
WODB & 2 & -- & 1 & 1 & 4 \\
Totals & 2 & 1 & 1 & 2 & 6 \\
\hline
\end{tabular}

It is worth noting that the WODB IA lends itself particularly well to the move T Makes

Connection, because the structure of the IA focuses on comparing and contrasting objects from a single mathematical category (e.g., systems of linear equations, equations of linear functions). Therefore, while the data in Table 14 makes it seem as though TCs increased their use of $T$ Makes Connection, at least some of that increase would be attributed to the structure of the IA.

TCs made connections a total of six times in the methods class. Of these instances, three were connections between two different student ideas. These three connections occurred throughout the semester: once during GOP, once during Card Sort, and once during WODB. In these instances, as well as one of the remaining instances, TCs seemed to make a connection in 
an effort to help students identify and correct a mistake. Consider the following excerpt from Charity's WODB rehearsal, which was focused on systems of linear equations:

T: How would we know that this is infinitely many solutions? If I were to graph this, what would this look like?

Bridget: The same.

[slight pause in discussion]

Stephanie: When I did it, I put them in $y=m x+b$ form. I got them both to equal $y=2 x+2$ when I did that.

T: $\quad$ Okay. So if they're both $y=2 x+2$, what does that look like if I graph it?

Bridget: They're on top of each other.

T: $\quad$ They're on top of each other. So what does that mean? If we said down here (pointing to a system that had previously been discussed), that one solution means where they intersect, what does that mean if they're laying on top of each other?

Bridget: It means every point [on the line] would be a solution. If they're on top of each other, it means that they're always touching.

When Charity asked students to consider a system of linear equations with infinitely many solutions during her WODB rehearsal, the students disagreed at first on what the solution(s) to the system should be. (Grace and Julian offered the idea that the system would have a single solution, while Bridget and Stephanie found the system to have infinitely many solutions.) Eventually, Charity connected an idea from a different system (that had only one solution) to help students understand why the system being discussed had infinitely many solutions. 
Charity mentioned this moment in her video annotation, but she did not seem aware of the connection she had made: "This tile was used to reach the goal of 'identical/equivalent lines result in infinitely many solutions'." Thus, she saw that she was working toward her goal, but she did not seem to notice how she worked toward the goal by making a connection between student ideas. An awareness of how Charity connected student ideas to help reach her goal is important to her development - if she does not recognize her use of T Makes Connection or the affordances of enacting the move, it is unlikely that she will identify moments in which it would be advantageous to enact this move responsively in the future.

The remaining three instances of T Makes Connection saw TCs drawing connections between a student's idea and a mathematical fact. Consider the following example from Grace's WODB rehearsal:

T: Charity, which equation did you choose?

Charity: $y=x$.

T: $\quad y=x$. okay. So why did it seem different than the others?

Charity: It's the only one that would be linear, so it's the only one that doesn't curve.

T: $\quad$ Okay.

Charity: And it's the only one without a power or exponent.

T: $\quad$ Okay...Bridget, did you agree with that?

Bridget: When she first said it, no. But then she pointed out that the $\sqrt{x}$ would still be $x^{\frac{1}{2}}$

T: Okay, but can't we just put a little 1 right here [as an exponent on $x$ in $y=x]$ ? What do we think about that? Can we do that?

Charity: Yes, you could. 
T: $\quad$ So you went ahead and said this one could be $y=x^{\frac{1}{2}}$ ?

In this instance, Grace drew a connection from the student's idea (i.e., $y=\sqrt{x}$ could be written as $y=x^{\frac{1}{2}}$ ) to the established mathematical fact that any variable with no exponent displayed actually has an exponent of 1 . This served to make apparent that, although it was not displayed on the board, $x$ actually does have an exponent in the equation $y=x$. Similar to Charity's example, making this connection helped Grace move toward one of her goals, which was to have students identify the powers of power functions.

The infrequency with which this move was enacted speaks to its difficulty-it requires TCs to make decisions in the moment not only about how to keep the IA moving toward the goal, but also how to draw connections among student ideas, which may or may not have been anticipated by the TC. Enacting this move well involves a great deal of complexity, and as such, TEs should not expect TCs to master it in a one-semester methods course. In addition to the complexity associated with enacting this move responsively, TCs' awareness of their enactments of the move must also be considered. The TCs in this study did not necessarily demonstrate development as responsive teachers through the use T Makes Connection, even though their enactments of the move were considered responsive. Their lack of awareness that they had enacted the move suggests that they enacted the move by chance, rather than in judicious ways. Without a solid understanding of when and why they enacted the move in responsive ways, the TCs cannot grow in their understanding of responsive teaching. These findings demonstrate that an increased focus on this move, by both TCs and TEs, will be required to help TCs develop skill in identifying connections between student ideas and making them public. 
Learning to attend to student ideas, both in planning and during class discussions, is an important part of becoming a responsive teacher. In their planning, the TCs in this study anticipated student reasoning and considered ways to foreground students' ideas during class discussions. During rehearsals, they developed skill in maintaining a focus on student ideas, as evidenced by their continued responsive use of the teaching moves T Revoices, and Ask Different S to Revoice. Although they were not always aware of their use of the move T Makes Connection, TCs' enactments of the move still led to responsive interactions with students by making connections between students' ideas or between a student's idea and an established mathematical fact. While TCs found success in enacting revoicing and connecting moves responsively, an examination of TCs' developing skill in responsive teaching requires a consideration of their shortcomings as well. In the next section, I highlight teaching moves that are positioned as responsive in the literature, but which were not always enacted responsively by the TCs in my study.

Potentially responsive moves not enacted responsively. In Chapter 2, I referenced a study by Milewski and Strickland (2016) in which they identified a responding framework that named responsive teaching moves as a means of analyzing and discussing their instructional practice (see Table 1). These moves were identified as responsive by a group of in-service mathematics and science teachers, along with their professional development facilitators. Despite their classification as responsive teaching moves, the TCs in my study demonstrated that teaching moves with the potential to be responsive are not always enacted in responsive ways. In the sections that follow, I provide examples of three moves identified as responsive by Milewski and Strickland (2016), but which were not always enacted responsively by the TCs in my study: T Clarifies, Ask S if they Agree/Disagree, and Ask Different S to Add On. 
T Clarifies. In terms of the adapted framework in Table 1 from Milewski and Strickland (2016), T Clarifies would fall under the category of Teacher Revoice. In my coding, I drew a slight distinction between $T$ Revoices and $T$ Clarifies. Recall that revoicing a student idea involves restating the idea without adding any information. When clarifying a student idea, however, the TC might revoice part or all of the idea, while also adding details as necessary. This move has the potential to be responsive because, ideally, a teacher would keep the student's idea central to the class discussion, even as they add words or information that might make the idea more understandable for the class as a whole. Although the TCs in this study appeared to attempt to foreground students' ideas through the use of $T$ Clarifies, their enactments were not always responsive as they added information to clarify those ideas.

TCs enacted the move $T$ Clarifies 13 times over the course of the semester, but only six of these instances were considered responsive. Responsive instances of the move kept student reasoning central by (1) ensuring proper recording of an idea, (2) graphing an equation to show a property described by a student, or (3) ensuring that the class was focused on the proper tile (during WODB only). The other seven instances were labeled as not responsive. In these instances, when TCs inserted details to clarify a student's idea, they had a tendency to shift the focus from the student's idea to their own. Often, the additional information from the TC seemed to be an effort to ensure that the mathematical goals for the IA were achieved, which is commendable. However, when these insertions did not allow for student input, they were rendered unresponsive. Enactments of $T$ Clarifies were also considered unresponsive if the TC made an inference about student reasoning, instead of asking how the student arrived at their conclusion. Table 15 displays the number of responsive and unresponsive instances of 
$T$ Clarifies, organized by TC and IA. Figure 18 provides examples of this move being used in both responsive and unresponsive ways. 
Table 15

Responsive and Unresponsive Instances of T Clarifies

\begin{tabular}{|c|c|c|c|c|c|c|c|c|c|c|}
\hline \multirow[b]{2}{*}{ Demo/IA } & \multicolumn{2}{|c|}{ Julian } & \multicolumn{2}{|c|}{ Bridget } & \multicolumn{2}{|c|}{ Charity } & \multicolumn{2}{|c|}{ Grace } & \multicolumn{2}{|c|}{ Totals } \\
\hline & Resp. & Unresp. & Resp. & Unresp. & Resp. & Unresp. & Resp. & Unresp. & Resp. & Unresp. \\
\hline Teaching Demo & -- & -- & -- & -- & -- & -- & -- & -- & 0 & 0 \\
\hline GOP & -- & 1 & -- & -- & -- & -- & -- & 1 & 0 & 2 \\
\hline CthenC & -- & -- & 2 & -- & -- & -- & -- & -- & 2 & 0 \\
\hline Card Sort & 1 & -- & -- & 1 & -- & 1 & -- & 1 & 1 & 3 \\
\hline WODB & 1 & 1 & -- & 1 & -- & -- & 2 & -- & 3 & 2 \\
\hline Totals & 2 & 2 & 2 & 2 & 0 & 1 & 2 & 2 & 6 & 7 \\
\hline
\end{tabular}




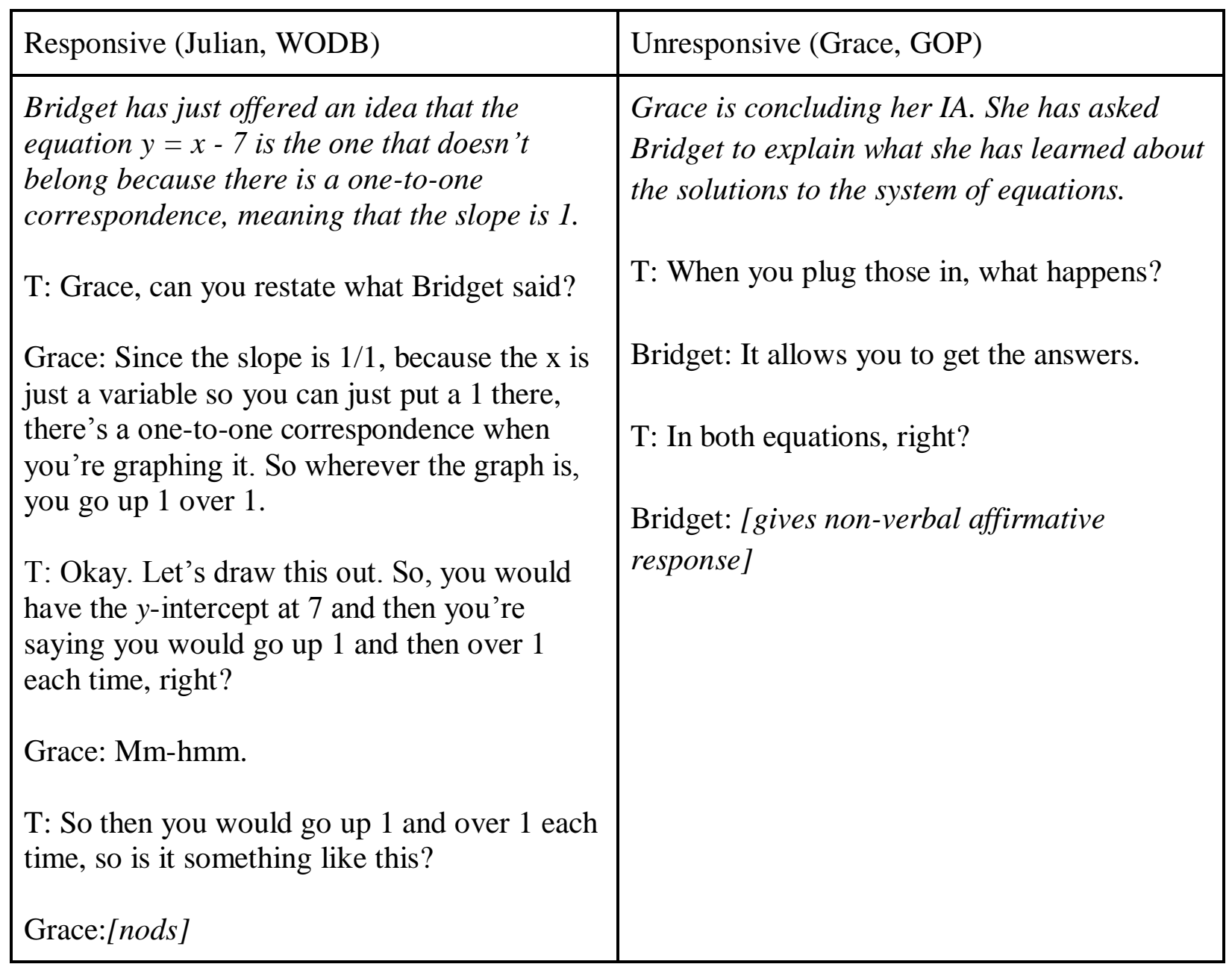

Figure 18. Responsive and Unresponsive Uses of T Clarifies

Julian elicited an idea from Bridget about a one-to-one correspondence and asked Grace to revoice it. (Note that neither student was describing the function as being one-to-one; they were describing "up 1, over 1" as a one-to-one correspondence.) The imprecise language used by both Bridget and Grace left Julian with an idea that had still not been clearly explained. As a result, he chose to clarify the idea by graphing the equation, being careful to use only the ideas put forward by the students to create the graph. He also confirmed with Grace that he had represented her ideas correctly. This instance was considered responsive for its attention to keeping student thinking central to the discussion. 
In contrast, when Grace asked, "So it has to be true for both equations, right?", she ensured that the words from her mathematical goal were uttered during the IA, but the exchange lacks evidence that students understood the statement or why it must be true. As a result, this instance was considered unresponsive. In her video annotation, Grace highlighted this exchange as part of a longer instance that she saw as focused on her goals for the IA. She wrote, "These concluding questions allowed the students to have the opportunity to summarize what was learned." Grace's other questions during the longer instance did foreground student ideas while also reiterating the goals for her IA. However, she did not seem to realize that this particular question, "In both equations, right?", did not surface student ideas, but instead, led students to the answer she was seeking.

In their attempts to enact the move $T$ Clarifies, TCs had a tendency to add information in ways that shifted the focus of the discussion from student ideas to the teacher's idea. Although this teaching move is classified as responsive in the literature, it was enacted unresponsively about half the time. Thus, enacting a move that appears to be responsive on the surface does not necessarily indicate development in responsive teaching. While the responsiveness of $T$ Clarifies relies mainly on the TCs enactment of the move itself, the responsiveness of two other teaching moves, Ask S if they Agree/Disagree and Ask Different $S$ to Add On, also rely on what follows TCs' enactment of the move. In the next section, I examine the first of these two moves, Ask $S$ if they Agree/Disagree.

Ask $S$ if They Agree/Disagree. Milewski and Strickland (2016) position Ask $S$ if they Agree/Disagree as a move which invites students to interact with each other's ideas, giving it the potential to be responsive. When enacting this move, the teacher asks a student (or the entire class) if they agree or disagree with something that has been said or written. However, this move 
really cannot be responsive if used in isolation; knowing whether students agree or disagree without knowing why is of little benefit (Chapin, O'Connor, \& Anderson, 2013; Smith \& Stein, 2011). Thus, the determination of the responsiveness relies heavily upon the response of the student(s) and the follow-up move used by the teacher (i.e., Does the teacher ask students why they agree or disagree, provide an explanation, or just move on?).

The only two TCs to enact Ask $S$ if they Agree/Disagree in methods were Bridget and Grace. They used the move six times during their IAs in methods, with three of Grace's enactments being considered responsive. Ask $S$ if they Agree/Disagree did not promote the responsive teaching practice of orienting students to each other's ideas on its own; the determination of responsiveness was dependent upon what followed. Table 16 displays a count of responsive and unresponsive instances of this move, organized by TC and IA. 
Table 16

Responsive and Unresponsive Instances of Ask S if they Agree/Disagree

\begin{tabular}{|c|c|c|c|c|c|c|c|c|c|c|}
\hline Demo/IA & \multicolumn{2}{|c|}{ Julian } & \multicolumn{2}{|c|}{ Bridget } & \multicolumn{2}{|c|}{ Charity } & \multicolumn{2}{|c|}{ Grace } & \multicolumn{2}{|c|}{ Totals } \\
\hline GOP & -- & -- & -- & 1 & -- & -- & 1 & -- & 0 & 2 \\
\hline CthenC & -- & -- & -- & -- & -- & -- & 1 & 1 & 1 & 1 \\
\hline WODB & -- & -- & -- & -- & -- & -- & 1 & -- & 1 & 0 \\
\hline Totals & 0 & 0 & 0 & 2 & 0 & 0 & 3 & 1 & 3 & 3 \\
\hline
\end{tabular}


In methods, Bridget and Grace always asked some variation of "Do you agree?" without offering the option to disagree. Chapin and colleagues (2013) note that this strategy of enacting Ask $S$ if they Agree/Disagree does not allow students to engage deeply with one another's reasoning. In each enactment of this move in methods, the original student had provided mathematically correct reasoning. By not offering students the option to disagree, Bridget and Grace seemed to be seeking support for those correct ideas with little concern as to why students agreed. In these instances, ensuring that students agreed with the idea was ultimately unnecessary; any misunderstanding or disagreement would have become evident as a result of a follow-up move (e.g., asking why a student agreed or disagreed). The examples in Figure 19 illustrate responsive and unresponsive enactments of this move.

\begin{tabular}{|c|c|}
\hline Responsive Exchange (Grace, GOP) & Unresponsive Exchange (Bridget, Card Sort) \\
\hline $\begin{array}{l}\text { T: So out of these two methods here, which } \\
\text { one do you think is most ideal for this system? }\end{array}$ & $\mathrm{T}$ : What did your group think about $y=\frac{4}{x} ?$ \\
\hline Bridget: Elimination. & Grace: We put it as a non-example. \\
\hline T: Do you agree? & T: Why? \\
\hline Charity: (nods) & $\begin{array}{l}\text { Grace: Because when you multiply that } x \text {, it's } \\
x y=4 \text {. So } x \text { is on the bottom. }\end{array}$ \\
\hline T: Why? & T: Any other reasons? \\
\hline $\begin{array}{l}\text { Charity: I mean, I'd much rather use } \\
\text { elimination. It goes quicker. }\end{array}$ & Grace: (shakes head) \\
\hline $\begin{array}{l}\text { T: When we look at the system itself, how do } \\
\text { we know that elimination was the best way to } \\
\text { go, without even starting? }\end{array}$ & $\begin{array}{l}\text { T: (to second group) Did you guys agree? } \\
\text { Students in second group: (nods) }\end{array}$ \\
\hline $\begin{array}{l}\text { Charity: Because you have a } 2 y \text { and a }-2 y \text {, so } \\
\text { those cancel. }\end{array}$ & T: All right. \\
\hline $\begin{array}{l}\text { T: So the coefficients are cancelling. Does } \\
\text { everyone see that? }\end{array}$ & \\
\hline
\end{tabular}

Figure 19. Responsive and Unresponsive Instances of Ask S if they Agree/Disagree 
In Grace's GOP rehearsal, she asked Charity to explain why she agreed with Bridget. This eventually led Grace to ask a follow-up question encouraging Charity to articulate how we can determine the most efficient method to solve a system. Grace described her perception of this moment in her video annotation: "Instead of the instructor saying why the elimination method was the most ideal, the student was given the opportunity to share her thoughts on the matter with the class." Grace's continued focus on student reasoning made this instance responsive.

Alternatively, in Bridget's Card Sort rehearsal, it is not clear whether Bridget is asking the second group if they (1) agree that this card is a non-example or (2) agree with the reasoning given by Grace explaining why the card was a non-example. Either way, Bridget does not ask the second group to explain why they agree; upon confirmation of agreement, she moved on to discuss another card. In this case, student reasoning did not remain central to the discussion, which made the instance unresponsive.

Ask $S$ if they Agree/Disagree is a teaching move that cannot be responsive on its own. Instead, as evidenced by Grace's enactments, asking students to explain why they agreed with the idea being discussed was essential to using this move as part of a responsive exchange. The TCs did not necessarily develop skill in enacting this move responsively, though more attention to the importance of the follow-up move on the part of the TE and TCs would support that development. Finally, I discuss a second move whose responsiveness relies not only on the TC's enactment, but also upon the student response: Ask Different $S$ to Add On.

Ask Different S to Add On. Ask Different S to Add On is another move positioned as responsive in the literature, but it was never enacted responsively by TCs in this study. Milewski and Strickland (2016) positioned this move as responsive in the sense that it provides an opportunity to orient students to one another's ideas. Ideally, by asking a student to add on, the 
teacher would keep the original student's idea as the focus of the discussion while a second student contributed additional ideas or details related to the original idea (rather than introducing a completely new thought). It is not clear why the TCs in this study chose to ask a student to add on during their rehearsals, as none of them discussed this move in their interviews or video annotations. However, based on the timing of their enactments, it seems that in each case the TC used the move to ensure that every student in a particular group had an opportunity to speak.

TCs enacted this move only four times during methods. None of the enactments were considered responsive. In each instance, the teacher was calling on a different member of the same group, which immediately hindered the potential to orient students to one another's ideas; since the students who were grouped together had previously discussed the ideas, they each already had an understanding of the idea. It would have been more responsive to ask a student from a different group if they had anything to add, to ensure that someone from outside the original group understood what was being discussed. As a result, none of these instances were considered responsive - they did not orient students to one another's thinking. Table 17 displays the number of instances of Ask Different $S$ to Add On, organized by TC and IA. Figure 20 shows excerpts of two different types of unresponsive instances of this move. 
Table 17

Responsive and Unresponsive Instances of Ask Different S to Add On

\begin{tabular}{|c|c|c|c|c|c|c|c|c|c|c|}
\hline \multirow[b]{2}{*}{ Demo/IA } & \multicolumn{2}{|c|}{ Julian } & \multicolumn{2}{|c|}{ Bridget } & \multicolumn{2}{|c|}{ Charity } & \multicolumn{2}{|c|}{ Grace } & \multicolumn{2}{|c|}{ Totals } \\
\hline & Resp. & Unresp. & Resp. & Unresp. & Resp. & Unresp. & Resp. & Unresp. & Resp. & Unresp. \\
\hline Teaching Demo & -- & -- & -- & -- & -- & -- & -- & -- & 0 & 0 \\
\hline GOP & -- & -- & -- & -- & -- & -- & -- & -- & 0 & 0 \\
\hline CthenC & -- & -- & -- & -- & -- & -- & -- & 1 & 0 & 1 \\
\hline Card Sort & -- & -- & -- & -- & -- & -- & -- & 1 & 0 & 1 \\
\hline WODB & -- & 1 & -- & 1 & -- & -- & -- & -- & 0 & 2 \\
\hline Totals & 0 & 1 & 0 & 1 & 0 & 0 & 0 & 2 & 0 & 4 \\
\hline
\end{tabular}




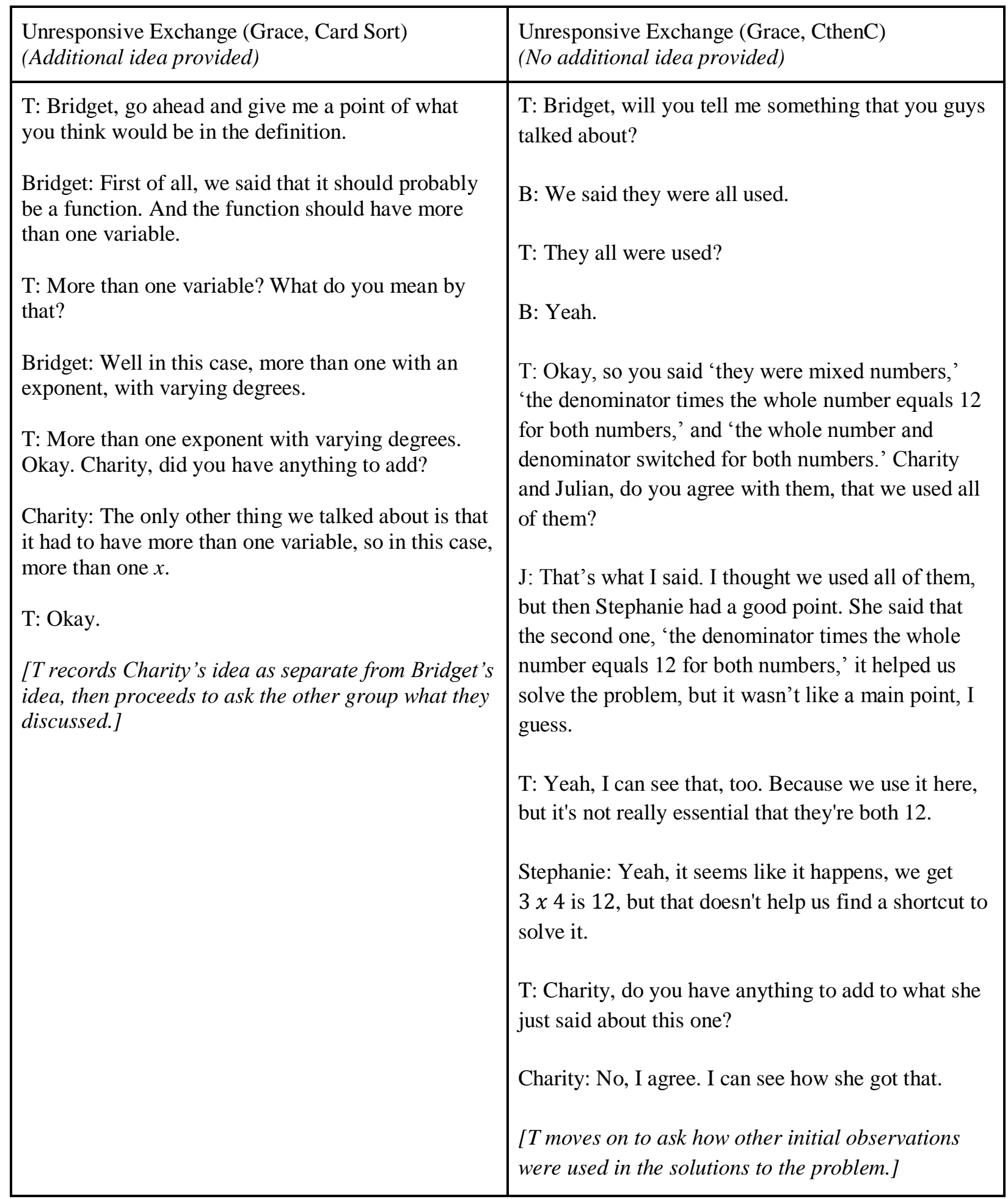

Figure 20. Two Types of Unresponsive Instances of Ask $S$ if they Agree/Disagree 
The instance of Ask Different S to Add On from Grace's Card Sort rehearsal was considered unresponsive because Grace called on Charity, a member of Bridget's group, to add on to Bridget's idea. Charity explains some additional details to help clarify Bridget's imprecise statement, which was helpful. However, this move did not serve to orient Charity to Bridget's thinking because Charity and Bridget had already discussed their ideas.

The instance of Ask Different S to Add On from Grace's CthenC rehearsal was also considered unresponsive, but for a different reason. It is not clear what Grace hoped to gain by asking Charity if she had anything to add. At that point in the discussion, Charity was the only student who had not yet contributed an idea; it seems like Grace may have called on Charity as more of a general invitation to participate in the conversation, rather than a specific invitation to add on to Stephanie's idea. This excerpt illustrates that the responsiveness of Ask Different $S$ to Add On relies not only on the TC's enactment of the move, but also on the student's response to the request. When Charity had nothing to add, that signaled the end of the exchange and Grace moved on with the IA, making it difficult for that particular enactment of Ask Different $S$ to Add On to be responsive.

The enactments of Ask Different $S$ to Add On during this study demonstrate that while the move could invite a student to interact with another student's idea, TCs can also use the move in other ways, such as a general invitation to join the discussion. The responsiveness of this move is heavily dependent upon the choice of the student called upon by the teacher (i.e., calling on someone from a different group) and the student's response. The evidence suggests that this move is difficult to enact responsively, unless the $\mathrm{TC}$ has given deliberate attention to the purpose for requesting additional ideas. Although TCs did enact Ask Different S to Add On more often as the semester progressed, this is not necessarily evidence of TC development in 
responsive teaching — simply using pre-defined responsive teaching moves does not mean they are used in responsive ways. However, TCs' increased use of the move suggests that they are attempting a wider variety of moves, which does indicate some degree of development. What remains for TCs to develop, then, is a more focused intent and a more responsive execution of Ask Different $S$ to Add On.

As evidenced in the discussion of T Clarifies, Ask $S$ if they Agree/Disagree, and Ask Different $S$ to Add On, the potential of a teaching move to be responsive does not guarantee that it will be enacted in responsive ways. The responsiveness of the moves depended on (1) when and how the teacher enacts the move, (2) how the student responds, and (3) how the teacher proceeds following the student response. This speaks to the complexity of this work and the need for TCs to have multiple opportunities to rehearse and reflect as they develop responsive teaching skills. In order to develop skill in enacting these moves responsively, TCs should carefully consider what they hope to achieve by enacting these moves. The TCs in this study did not seem to be particularly deliberate in their enactments of these three moves - they rarely spoke about them in their interviews or in their video annotations. In the next section, I discuss two other teaching moves that TCs enacted habitually, rather than deliberately: T Repeats and Anything Else.

Moves enacted habitually. As discussed in Chapter 3, individual moves were initially identified as responsive based solely on the enactment of the move with little or no consideration of the intent or result of the move. Thus, even moves that TCs enacted out of habit (rather than as a result of deliberate attention to responsiveness) were initially coded during each S-T exchange. In later phases of analysis, responsiveness was determined by considering the sequence of moves to which it belonged, which involved a consideration of the intent, enactment, and result of the 
move. In the sections that follow, I discuss two teaching moves that TCs seemed to enact out of habit: T Repeats and Anything Else.

T Repeats. While similar to the moves T Revoices and T Clarifies, which were discussed in previous sections of this chapter, T Repeats was defined with one subtle differenceteacher repeats a student's idea verbatim, without alterations. This is a ubiquitous move in teaching; many teachers repeat students' ideas or statements more out of habit than to achieve any particular purpose. With this in mind, it is not surprising that TCs in this study repeated student ideas frequently_49 times in methods — with Julian and Grace using the move nearly four times as often as Bridget and Charity. Table 18 displays the counts of responsive and unresponsive instances of this move. 
Table 18

Responsive and Unresponsive Instances of T Repeats

\begin{tabular}{|c|c|c|c|c|c|c|c|c|c|c|}
\hline \multirow[b]{2}{*}{ Demo/IA } & \multicolumn{2}{|c|}{ Julian } & \multicolumn{2}{|c|}{ Bridget } & \multicolumn{2}{|c|}{ Charity } & \multicolumn{2}{|c|}{ Grace } & \multicolumn{2}{|c|}{ Totals } \\
\hline & Resp. & Unresp. & Resp. & Unresp. & Resp. & Unresp. & Resp. & Unresp. & Resp. & Unresp. \\
\hline Teaching Demo & -- & -- & -- & -- & -- & 1 & -- & 4 & 0 & 5 \\
\hline GOP & -- & 1 & 1 & 1 & 1 & 1 & 1 & 3 & 3 & 6 \\
\hline CthenC & 2 & -- & 1 & -- & -- & 1 & 3 & 1 & 6 & 2 \\
\hline Card Sort & -- & 12 & 2 & -- & -- & -- & 3 & 1 & 5 & 13 \\
\hline WODB & 4 & 2 & -- & 1 & 1 & -- & -- & 1 & 5 & 4 \\
\hline Totals & 6 & 15 & 4 & 2 & 2 & 3 & 7 & 10 & 19 & 30 \\
\hline
\end{tabular}


T Repeats was considered responsive only when the TC repeated a student idea to ensure that it was properly recorded on the board; this accounted for only 19 instances of 49 . The other 30 instances of this move were considered unresponsive because they did not serve to maintain a focus on student reasoning. It is worth noting, however, that unresponsive instances of repeating student ideas did not necessarily prevent S-T exchanges from being responsive overall. Since all of the responsive instances of $T$ Repeats occurred when the TC wanted to ensure proper recording, I will focus the remaining discussion on unresponsive instances of the move.

TCs sometimes appeared to enact $T$ Repeats unresponsively as a means of giving themselves time to decide what to say or do next. This usually happened when a student gave an incorrect answer. Consider the following example from Grace's GOP rehearsal. At this point in the class discussion, Charity had just explained the steps she used to solve a system of linear equations by elimination, though she did not name the method she used. Grace is asking the class to name the solution method:

$\mathrm{T}$ : What is this called?

Bridget: Substitution.

T: [slowly repeats] Substitution. [slight pause] Are we substituting here, at this point?

In this instance, Grace repeated Bridget's incorrect answer slowly, then paused as if she needed a moment to decide how to proceed. She acknowledged this in her video annotation:

This was a challenging moment because I did not initially know what to say. I think a better response would have been to ask if the class agreed or not and have a student that disagreed explain her reasoning. Then I could have asked the student that initially disagreed if she agrees and to explain why the method is called elimination. 
Notice that Grace did begin to work toward eliciting Bridget's reasoning by asking where she saw the substitution occurring, which is a responsive teaching move. However, her repeating of Bridget's incorrect answer did not contribute to the responsiveness of the exchange. In fact, Grace's slow repeat might have served as a cue that something was incorrect, which could have made the instance decidedly unresponsive, as the attention was turned toward the teacher's thinking. Instead, Grace could have achieved the same purpose by simply pausing for a moment to decide upon her next question.

Other unresponsive instances of $T$ Repeats seemed to arise simply out of habit. Consider the following example from Julian's Card Sort rehearsal:

T: $\quad$ How about Charity and Bridget? What is one graph that you chose as an example?

Charity: Graph B.

T: $\quad$ Graph B? Okay. Why did you pick Graph B?

Here, Julian seemed to have a natural inclination to repeat Charity's response. He went on to use T Repeats in similar ways 11 more times during his Card Sort rehearsal alone, which further supports the idea that, for Julian, this was more of a habit than a deliberate responsive teaching move.

While it could be argued that Julian is maintaining a focus on Charity's response by repeating it, I contend that, although not especially detrimental, Julian's repeating of her answer is ultimately unnecessary. Instead, he maintains a focus on Charity's reasoning by using different teaching moves a little later in the exchange. Consider this slightly longer excerpt, which includes the teaching moves Julian enacted after he repeated Charity's response: 
T: How about Charity and Bridget? What is one graph that you chose as an example?

Charity: Graph B.

T: $\quad$ Graph B? Okay. Why did you pick Graph B?

Charity: Because it's a straight line and there's a constant slope of 0 .

T: $\quad$ [records] You said it's a constant slope at where?

Charity: It's a constant slope of 0 .

T: $\quad$ Is there anything else?

Charity: There's not a curve.

This S-T exchange as a whole would be considered responsive for its focus on Charity's reasoning throughout. Julian remained focused on asking Charity to provide her reasons for choosing Graph B and he even asked her to clarify her response to ensure he recorded it properly. Thus, while not especially productive, Julian's use of T Repeats was also not necessarily detrimental to the responsiveness of the instance.

Repeating student ideas, while not usually responsive, is also not decidedly unresponsive. As TCs develop skill in responsive teaching, they should be mindful of their enactments of T Repeats, but mainly to ensure that those enactments do not become a distraction during the IA. Similarly, TCs enacted one other move, asking some variation of "Anything else?" with little impact on the responsiveness of an exchange.

Anything Else. Another move which was enacted frequently, but which was largely considered unresponsive, occurred when TCs asked some version of the question, "Anything else?" as a way to invite new ideas from any student in the class. Typically, the planning materials for IAs encourage TCs to be selective as they decide who to call on to share ideas, as a 
means of keeping the IA focused on the goals. Broadly asking, "Anything else?" provides an opportunity for students to share ideas that might steer the discussion away from the instructional goals. However, TCs seemed to naturally enact this move on their own; I did not suggest it as a responsive teaching move.

TCs asked some version of "Anything Else?" regularly during the first two IAs, causing it to become a topic of conversation for our whole-class reflection after the second IA, CthenC. During that discussion, TCs took the stance that this move can sometimes be productive because the teacher is trying to provide additional time and opportunities for students to share their ideas. Charity suggested that asking "Anything else?" might provide students with a little more time to think, while Bridget noted that inviting student ideas in this way might surface a "really good" idea that the teacher did not notice earlier in the IA. Grace stated that, in CthenC specifically, it could be good to broadly ask for other ideas during the first part of the IA, when the teacher is eliciting observations about the problem. In response, I attempted to help TCs consider their intent in asking the question in order to determine whether or not it is likely to be productive.

In my analysis, I considered both the intent and the outcome of asking such a question. These considerations led to two different labels in my research. The first was T Elicits Other Ideas from $S$. All instances of this move were considered productive because the TC was able to successfully elicit a new student idea. As explained in Chapter 3, this label evolved over the course of the semester and eventually led to a second label: T Invites Other Ideas from S - None Elicited. For the remainder of this section, I will use Anything Else to refer to the enactments of moves with either of these two labels.

TCs enacted Anything Else 60 times during methods, with only 20 instances successfully eliciting a new student idea. Table 19 displays a count of when this move was used, organized by 
TC and IA. (The numbers in Table 19 represent S-T exchanges when the label Anything Else occurred; they do not represent the number of times a TC asked, “Anything else?" during an IA.) 
Table 19

Responsive and Unresponsive Instances of Anything Else

\begin{tabular}{|c|c|c|c|c|c|c|c|c|c|c|}
\hline Demo/IA & \multicolumn{2}{|c|}{ Julian } & \multicolumn{2}{|c|}{ Bridget } & \multicolumn{2}{|c|}{ Charity } & \multicolumn{2}{|c|}{ Grace } & \multicolumn{2}{|c|}{ Totals } \\
\hline GOP & -- & -- & -- & -- & -- & 1 & -- & -- & 0 & 1 \\
\hline CthenC & 1 & 1 & -- & -- & 1 & 2 & 2 & 1 & 4 & 4 \\
\hline WODB & 1 & 5 & 1 & 5 & 3 & 1 & 4 & 3 & 12 & 11 \\
\hline Totals & 7 & 10 & 3 & 11 & 5 & 11 & 8 & 8 & 23 & 37 \\
\hline
\end{tabular}

Note. These numbers represent S-T exchanges when the label Anything Else occurred. They do not represent the number of times a TC asked, "Anything else?" during a given IA. 
Generally, Anything Else was considered responsive if the TC successfully elicited a new idea from a student as a result. Three of the responsive instances shown in the table represent moments when a TC invited new ideas with no initial response from the student, but persistence eventually led to the elicitation of a new idea.

TCs' unresponsive enactments of the move Anything Else seemed to result from a natural inclination to ask such a question at different points during the IA. For example, TCs sometimes asked "Anything else?" or "Any questions?" at the end of an IA. When they received no response, this signaled the end of the IA. Similarly, as evidenced by Grace's comment during our whole-class reflection on CthenC, when TCs were eliciting observations about a problem, card, or tile, they were more likely to ask "Anything else?" before moving on with the IA, as a way to ensure that all student ideas had been elicited. The structures of the CthenC, Card Sort, and WODB IAs are likely partially responsible for these instances of Anything Else. These three IAs ask students to observe something, leaving more possibilities for students to share different ideas (as opposed to Going Over a Problem, where noticings are less central to the discussion).

In some cases, the teacher invited other ideas and successfully elicited two or three new ideas in succession. Then, when the teacher invited additional ideas, perhaps anticipating more responses, they received no response from the students. In these cases, it seems as though the TCs were simply ensuring they had elicited all student ideas. As an example, consider this excerpt from Charity's CthenC rehearsal, as she was eliciting observations about the problem.

T: Bridget, what is something you noticed?

Bridget: All the denominators are multiples of 11.

T: Julian, what did you notice?

Julian: Actually, we talked about the same thing. 
T: $\quad$ Same thing? Okay. Is there anything else anybody noticed?

Bridget: All the fractions are under a half.

T: $\quad$ Anything else?

Stephanie: The numerators didn't have any common factors.

T: $\quad$ Anything else?

[Receiving no response to this final question led Charity to move on with the IA.]

Notice that Charity broadly invited other ideas three times during this instance. The first two times resulted in successful elicitations of new ideas. The third time, although she did not elicit anything new, it seems as though she was making an effort to elicit every possible idea before moving on with the rehearsal.

The majority of TCs' enactments of Anything Else were deemed unresponsive. They seemed to enact this move more out of habit than as a deliberate effort toward responsive teaching. It is important to note, however, that unproductive instances of this move were not necessarily detrimental to the responsiveness of the $\mathrm{S}$-T exchange as a whole. Nevertheless, it is worthwhile for TCs to be mindful of how they expect students to respond to such a question and how they plan to work toward the mathematical goal(s) using student responses.

T Repeats and Anything Else are two ubiquitous moves in teaching. TCs' enactments of these moves did not usually seem deliberate; instead, they seemed to have been enacted out of habit. TCs also did not use either of these moves more judiciously over time. In terms of responsiveness, these moves are neutral — they are rarely responsive on their own, but they do not compromise the responsiveness of an entire $\mathrm{S}-\mathrm{T}$ exchange. This conclusion is encouraging for TCs and TEs, providing evidence that, aside from excessive use, these moves require little 
attention, allowing for a more pointed focus on moves such as Ask Different $S$ to Revoice, which have more of a direct impact on responsive teaching.

Summary. In summary, TCs developed skill in responsive teaching by attending to student ideas in their planning, aided by the pre-planners and protocols, and during class discussions, through the use of revoicing and connecting moves. TCs also made attempts to enact moves that are positioned as responsive in the literature. However, they did not always enact these moves in responsive ways. In the context of a methods course, this is not necessarily problematic, as it presents opportunities for further discussion and fine tuning of the moves, though those opportunities were not always fully realized or leveraged during the semester. Finally, there are some moves that TCs seemed to use habitually. TCs did not use these moves more judiciously over time, but since their use does not significantly add or detract from the responsiveness of an entire exchange, they require less attention than other teaching moves that directly impact responsive teaching.

\section{RQ2: How Does Coaching in Methods Affect TCs' Responsive Teaching?}

As described in Chapter 2, coached rehearsals require more of TCs and TEs than "microteachings" or "run-throughs" of lessons. During a coached rehearsal, the TE, acting as the coach, may pause the IA to provide immediate feedback to the TC. This feedback may or may not require the TC to "replay" a particular teaching move or instance. Either way, one of the affordances of using coached rehearsals is that the feedback is public, allowing all TCs the opportunity to hear and learn from feedback given to their classmates. During a rehearsal, the TC leading the IA is also permitted to pause the rehearsal to ask questions of the coach. The immediate feedback that results from TCs' questions is also valuable, in that TCs have the 
opportunity to work through suggestions from the TE in real time, rather than receiving suggestions after the IA.

The coach is responsible for deciding when and how often to interject during an IA. Too many interjections may make it difficult for the TC to experience the continuity of the IA, thus detracting from the authenticity of the rehearsal. Too few interjections, however, may mean missing opportunities for TCs to improve their practice and for TEs to make certain aspects of practice more visible. As the TE of a very small methods course, I played the dual role of instructional coach and student during the rehearsals. I viewed TCs' rehearsals through the lens of responsive teaching, which almost certainly impacted my decisions about when to interject and what feedback to provide. In the sections that follow, I first provide an overview of the kinds of coaching interjections that occurred throughout the semester and consider the extent to which they were focused on responsive teaching. I then look more specifically at four types of coaching interjections and compare them to moves that TCs enacted on their own. Finally, I discuss TCs' perceptions of instructional coaching and indicate how they may affect the ways in which TCs take up coaching feedback.

Overview of C-T exchanges. My analyses revealed that 65 of the $106 \mathrm{C}-\mathrm{T}$ exchanges were focused on responsive teaching. Eight of these 65 instances were labeled as having more than one substance. (Recall that C-T instances were categorized by both substance and structure, as described in Chapter 3.) Interjections that were classified as not related to responsive teaching, while often focused on other important aspects of teaching (e.g., asking clear questions, giving understandable instructions, or correcting mathematical errors on the board), did not have TCs' responsiveness to student reasoning as their focus. This is to be expected and is consistent with other research (Lampert et al., 2013). Table 20 displays the number of instances focused on 
responsive teaching (labeled Resp.) and the number of instances not focused on responsive teaching (labeled Unresp.) for each structure of coaching interjection: Directive Feedback, C Compliments $T$, and T asks $C$ a Question. Further descriptions of how the coaching moves were labeled are found in Table 6. 
Table 20

Responsive and Unresponsive Substance Labels for C-T Instances, Organized by Structure

\begin{tabular}{|c|c|c|c|c|c|c|c|c|}
\hline \multirow[b]{2}{*}{ Substance of C-T Exchange } & \multicolumn{2}{|c|}{$\begin{array}{l}\text { Directive } \\
\text { Feedback }\end{array}$} & \multicolumn{2}{|c|}{$\underline{\text { C Compliments }^{\mathrm{a}}}$} & \multicolumn{2}{|c|}{$\begin{array}{c}\text { T asks C } \\
\text { a Question }\end{array}$} & \multicolumn{2}{|c|}{ Totals } \\
\hline & Resp. & Unresp. & Resp. & Unresp. & Resp. & Unresp. & Resp. & Unresp. \\
\hline C Comments on IA Protocol & 2 & 20 & -- & -- & 2 & 1 & 4 & 21 \\
\hline C Comments on T's Recording & 12 & 6 & 2 & -- & 1 & -- & 15 & 6 \\
\hline C Asks T to Highlight Math Idea & 6 & 1 & 1 & 1 & 1 & 1 & 8 & 3 \\
\hline C Asks T to Further Question S & 6 & -- & 3 & -- & 3 & -- & 12 & 0 \\
\hline C Asks T to Ask Open Questions & 6 & -- & -- & -- & 1 & -- & 7 & 0 \\
\hline C Asks T to Clarify Question & 2 & 4 & 1 & -- & -- & -- & 3 & 4 \\
\hline C Asks T to Give Think Time & 6 & 1 & -- & -- & -- & -- & 6 & 1 \\
\hline C Asks $\mathrm{T}$ to Let $\mathrm{S}$ Revoice & 6 & -- & -- & -- & -- & -- & 6 & 0 \\
\hline $\begin{array}{c}\text { C Helps with } \\
\text { Technology/Materials }\end{array}$ & -- & 5 & -- & -- & -- & -- & 0 & 5 \\
\hline Miscellaneous & 3 & 9 & 1 & -- & -- & 2 & 4 & 11 \\
\hline Totals & 49 & 46 & 8 & 1 & 8 & 4 & 65 & 51 \\
\hline
\end{tabular}

${ }^{\mathrm{a}}$ None of compliments given were for moves that had been requested by the coach. For example, the instances categorized as both $C$ compliments $T$ and $C$ Asks $T$ to Highlight Math Idea indicate that I complimented the TCs for highlighting a math idea on their own. 
Notice that only one-third of the C-T exchanges were not focused on responsive teaching, with only one substance never being focused on responsive teaching: $C$ helps with Technology/Materials. In instances with this label, I helped TCs resolve issues that arose with the computer, projector, or cards (during the Card Sort rehearsal). Three other substances (Comments on IA Protocol, C Asks T to Clarify Question, and Miscellaneous) describe instances that were usually not focused on responsive teaching; still, each of those substances was focused on responsive teaching in at least one instance. Thus, the substance of a C-T exchange does not necessarily indicate that those exchanges will always be focused on responsive teaching (or not); that determination is made by the conversation that takes place.

To illustrate this point, consider the substance label C Comments on IA Protocol. In C-T instances labeled with this substance, I was usually reminding TCs to enact part of the IA they had forgotten. Since these reminders were not focused on student thinking, they were not counted as being focused on responsive teaching. For example, during Julian's Card Sort rehearsal, I interjected to say, "You also might want to remind us that it's okay if we don't get them all sorted, because you're going to stop us [before we finish sorting].”

However, in two instances labeled C Comments on IA Protocol, my reminders about the protocol also served to help the TC focus on student thinking. One example occurred during Julian's GOP rehearsal. As students began working on the problem, he remained stationed at the front of the room. I interjected to say, "You want to be walking around to just see what [the students] are doing." TCs were instructed to do this in the protocol, but my reminder also encouraged Julian to take note of the approaches being used by students to solve the problem. As a result, this interjection was considered to be focused on responsive teaching. Consequently, it 
was the substance of an interjection, not the purpose itself that determined whether a particular C-T instance was focused on responsive teaching.

Because I viewed TCs' rehearsals with an eye toward responsiveness, it is expected that instances labeled $C$ Compliments $T$ would be focused on responsive teaching (e.g., "You're doing a good job of writing exactly what she says."). This kind of positive feedback is important to TC development, as it highlights a successful enactment of a responsive teaching move for all TCs.

Somewhat unexpectedly, instances of $T$ asks $C$ a Question were also primarily focused on responsive teaching. This provides evidence for their development as responsive teachers; it demonstrates their awareness of moves that were unresponsive (e.g., give students a word to think about) and their desire to enact responsive teaching moves instead, even though they are unsure of what those teaching moves should be. Some examples of these instances will be shared in the sections that follow.

The instances I have described thus far have focused on purposes of interjections which were not primarily focused on responsive teaching. However, a determination of how coaching affected TCs' responsive teaching is best made by considering interjections that were primarily focused on responsive teaching. In the sections that follow, I discuss coaching interjections with four different substances, each of which was focused on a particular teaching move: $C$ Asks $T$ to Give Think Time, C Comments on T's Recording, C Asks T to Further Question S, and C Asks T to Let S Revoice. I chose these four substances because, taken together, they span the interjections which were focused on responsive teaching. Along with these interjections, I also compare the results of these interjections to the instances when TCs enacted these moves on their own, as a means of providing evidence to show the impact of coaching. I then discuss TCs' 
perceptions related to instructional coaching and explain why their perceptions are an important consideration for TEs engaging in this work.

Comparing coaching interjections and TCs' enactments. One useful way to determine how coaching affects TCs' responsive teaching is to compare moves enacted as a result of coaching interjections to moves that TCs enacted on their own. In some cases, TCs seem to have enacted certain moves more frequently as a result of coaching. In other cases, TCs enacted particular moves on their own, but began to enact them in more responsive ways as a result of coaching. In the sections that follow, I examine four different types of coaching interjections and compare the moves TCs made in response to those interjections with moves TCs made on their own. The first two, (1) C Asks T to Give Think Time vs. T Prompts S to Take Think Time and (2) C Asks T to Let S Revoice vs. Ask Different $S$ to Revoice, involve teaching moves that the TCs did not enact at all during their Teaching Demos, which suggests that they needed guidance to even attempt these moves. The last two, (3) C Comments on T's Recording vs. T Records, and (4) $C$ Asks $T$ to Further Question $S$ vs. T Further Questions $S$ involve moves that the TCs readily enacted during their Teaching Demos. However, TCs required guidance in learning how to enact these moves in more responsive ways. I discuss instances of $T$ Asks $C$ a Question and $C$ Compliments $T$ as they relate to these four comparisons.

T Prompts $S$ to Take Think Time. T Prompts S to Take Think Time seemed to be a new teaching move for the TCs in this study, as none of them provided time for students to think about or solve problems on their own during their Teaching Demos. In their pre-interviews, the TCs made some comments to suggest that they viewed the act of teaching as consisting only of the time they spent at the board explaining the problems to students. For example, Julian remarked more than once during his interviews that he wanted to develop skill in "finding the 
right words to say" as he worked to "communicate content better." Grace described "voice fluctuations, [to] keep kids involved" as one of her strengths as a teacher. These comments suggest that the TCs initially viewed communicating the content as their primary teaching responsibility. This helps to explain why TCs mainly asked direct, closed-ended questions during their Teaching Demos (e.g., “And 3 x 4 is?”). However, these types of questions severely limited the TCs' abilities to gain a true sense of students' mathematical understanding; in order to understand students' thinking, students must first be given time to think.

In some instances, the IA protocols asked TCs to enact T Prompts $S$ to Take Think Time; in other instances, TCs enacted the move on their own or at the suggestion of the coach. Table 21 displays the number of enactments of this move when prompted by the IA protocol as compared with TCs' novel enactments of the move. 
Table 21

Instances of T Prompts S to Take Think Time (suggested in IA protocol vs. novel enactments)

\begin{tabular}{|c|c|c|c|c|c|c|c|c|c|c|}
\hline \multirow[b]{2}{*}{ Demo/IA } & \multicolumn{2}{|c|}{ Julian } & \multicolumn{2}{|c|}{ Bridget } & \multicolumn{2}{|c|}{ Charity } & \multicolumn{2}{|c|}{ Grace } & \multicolumn{2}{|c|}{ Totals } \\
\hline & IA & Novel & IA & Novel & IA & Novel & IA & Novel & IA & Novel \\
\hline Teaching Demo & & -- & -- & -- & -- & -- & -- & -- & 0 & 0 \\
\hline GOP & 2 & 1 & 1 & 5 & 2 & 6 & 2 & 1 & 7 & 13 \\
\hline CthenC & 5 & 1 & 8 & -- & 5 & -- & 7 & 2 & 25 & 3 \\
\hline Card Sort & 3 & -- & 2 & 3 & 2 & 3 & 2 & 4 & 9 & 10 \\
\hline WODB & 4 & -- & 8 & -- & 4 & -- & 3 & 2 & 19 & 2 \\
\hline Totals & 14 & 2 & 19 & 8 & 13 & 9 & 14 & 10 & 60 & 28 \\
\hline
\end{tabular}


TCs enacted T Prompts $S$ to Take Think Time 88 times during methods, with none of those instances occurring during their Teaching Demos. Sixty of those instances were the result of a suggestion in the IA protocol. The remaining 28 enactments were novel in that the TC or the coach identified salient moments when taking think time might be beneficial. Although the novel instances were in the minority, these are the moments I will discuss throughout the remainder of this section, as TCs' decisions to prompt think time on their own provide insight into their development as responsive teachers. Table 22 displays the number of responsive and unresponsive instances of TCs' novel enactments of T Prompts S to Take Think Time, organized by TC and IA. Table 22 does not include instances when the IA protocol suggested that the TC provide think time. 
Table 22

Novel Instances of T Prompts S to Take Think Time

\begin{tabular}{|c|c|c|c|c|c|c|c|c|c|c|}
\hline \multirow[b]{2}{*}{ Demo/IA } & \multicolumn{2}{|c|}{ Julian } & \multicolumn{2}{|c|}{ Bridget } & \multicolumn{2}{|c|}{ Charity } & \multicolumn{2}{|c|}{ Grace } & \multicolumn{2}{|c|}{ Totals } \\
\hline & Resp. & Unresp. & Resp. & Unresp. & Resp. & Unresp. & Resp. & Unresp. & Resp. & Unresp. \\
\hline Teaching Demo & -- & -- & -- & -- & -- & -- & -- & -- & 0 & 0 \\
\hline GOP & 1 & -- & 3 & 2 & 6 & -- & 1 & -- & 11 & 2 \\
\hline CthenC & 1 & -- & -- & -- & -- & -- & 2 & -- & 3 & 0 \\
\hline Card Sort & -- & -- & 3 & -- & 3 & -- & 4 & -- & 10 & 0 \\
\hline WODB & -- & -- & -- & -- & -- & -- & 2 & -- & 2 & 0 \\
\hline Totals & 0 & 0 & 5 & 1 & 8 & 0 & 8 & 0 & 26 & 2 \\
\hline
\end{tabular}

Note. Table does not include instances when the protocol suggested providing think time. 
There were only two unresponsive instances of T Prompts $S$ to Take Think Time during methods. In one instance, Bridget offered students time to think, but then immediately asked students to respond. In the other instance, Bridget assigned students values to plug into an equation. Since student reasoning was not central to the class discussion, this instance was considered unresponsive. In the remaining 26 instances of this move, TCs provided time for students to formulate ideas and/or discuss them with a classmate before sharing ideas in the group discussion.

TCs were more likely to invite students to take time to think about the problems before responding during their IA rehearsals, as opposed to the Teaching Demos. In part, this may have resulted from prompts in the IA protocols that reminded TCs to provide those opportunities. However, TCs made additional notes on their protocols, such as "Give think time (10-15 sec) to make sense of approach" and "Think-Pair-Share," that demonstrated their attention to this teaching move, even when the protocols did not call for it (see Figure 13). My coaching interjections further helped TCs decide when it might be productive to enact this move. Table 23 displays the number of $\mathrm{C}$ - $\mathrm{T}$ exchanges focused on prompting think time compared with the number of instances when TCs enacted this move on their own. 
Table 23

T Prompts S to Take Think Time (responsive instances only) vs. C Asks T to Give Think Time

\begin{tabular}{|c|c|c|c|c|c|c|c|c|c|c|}
\hline Demo/IA & \multicolumn{2}{|c|}{ Julian } & \multicolumn{2}{|c|}{ Bridget } & \multicolumn{2}{|c|}{ Charity } & \multicolumn{2}{|c|}{ Grace } & \multicolumn{2}{|c|}{ Totals } \\
\hline GOP & -- & 1 & 2 & 2 & 6 & -- & 1 & $1^{\mathrm{b}}$ & 9 & 4 \\
\hline CthenC & -- & 1 & --- & -- & -- & -- & 2 & -- & 2 & 1 \\
\hline WODB & -- & -- & -- & -- & -- & -- & 2 & -- & 2 & 0 \\
\hline Totals & 0 & 2 & 6 & 2 & 8 & 1 & 8 & 2 & 21 & 7 \\
\hline
\end{tabular}

Note. Table does not include instances when the protocol suggested providing think time.

${ }^{a}$ There were no coaching interjections at all during the Teaching Demos.

${ }^{\mathrm{b}}$ This instance did not result in an enactment of $T$ Prompts $S$ to Take Think Time. 
Over time, TCs required less intervention from the coach to provide students with time to think. Notice that, during the Card Sort IA, Bridget, Charity, and Grace enacted T Prompts $S$ to Take Think Time on their own more often than in previous IAs. Grace was the only TC to enact this move during WODB, but I attribute that more to the structure of the IA than a lack of TC development. In WODB, when no students have identified why a particular tile might be the one that doesn't belong, the protocol suggests that TCs provide students some time to think about why that particular tile doesn't belong. As Julian, Bridget, and Charity rehearsed WODB, there were no particularly salient moments for TCs to provide think time, besides those indicated in the IA protocol. This conclusion is supported by the lack of coaching interjections around this move during WODB.

The seven instances of $C$ Asks $T$ to Give Think Time occurred primarily at two different times in the IA: when it was time for TCs to elicit reflections as a closure and when TCs posed a question that students had difficulty answering immediately. In three instances, I suggested that TCs give think time before eliciting reflections at the end of an IA because TCs sometimes had a tendency to want to elicit students' reflections too quickly. As an example of this type of interjection regarding think time, consider this excerpt from Julian's GOP rehearsal:

T: $\quad$ So, looking at this. Looking at these problems, what did you guys learn about solving... equations like this? [short pause] Grace, what's something you learned?

Coach: ...They need a minute to just reflect, 'Oh, what did I learn about this?' So, give them a minute to think, and you can even give them a minute to talk, if you want. 
T: Talk in your group...talk about what you guys learned today. Whenever you're done, give me a thumbs up.

A similar instance occurred during Grace's GOP rehearsal, but her comments shed some light on why TCs had this tendency to expect quick responses from students-providing wait time is sometimes difficult or uncomfortable. Consider this excerpt from her rehearsal:

T: $\quad$ Charity, will you summarize when we are supposed to use the elimination method?

Charity: You can use elimination whenever you have coefficients in your system that will cancel each other out.

T: And Bridget, will you explain again what this answer means in terms of our equations.

Bridget: $\quad$ It means $x=4$ and $y=-4$.

T: $\quad$ And so when you plug those in, what happens?

Bridget: [long pause] It allows you to get the answers?

T: $\quad$ In both equations, right?

Coach: The only thing I might have suggested there is give them a minute to think about what you were asking. If you wanted to focus that last bit, where you had them reflect, that's fine, but you might have wanted to let them think a minute before you call on them.

T: That's the hardest part! 'Cause you're standing here like, 'I know you know it.'

Coach: So they're thinking about exactly how to say it...If you can just say, 'Take a minute to think about what you learned' or 'Take a minute to think about how 
you know elimination is the most efficient method.' You don't have to wait a really long time, just long enough to let people gather their thoughts.

During this instance, my interjection occurred after Grace asked the students to reflect. The exchange between Grace and Bridget reveals that Grace had something fairly specific in mind for these reflections and Bridget had some difficulty in expressing those ideas immediately. The exchange between Grace and me (the coach) allowed some insight into why this might be a difficult move for TCs to make and served as a reminder for me to keep encouraging them to provide think time before asking students to reflect.

In the remaining four interjections of $C$ Asks $T$ to Give Think Time, I suggested that TCs provide think time in moments when the TC asked a question (not as part of the wrap-up of the IA) and none of the students responded. In one such instance, Charity felt pressured to ask funneling questions (i.e., questions meant to lead students to a desired conclusion; NCTM, 2014) to help students say the word(s) that she had in mind. Consider this excerpt from her Card Sort rehearsal.

T: $\quad$ I'm going to go ahead and give you the definition of a linear function. A linear function is a function where for any two ordered pairs in the table, the ratio of the change in $y$ to the change in $x$ is constant. What do you think that means...the ratio of the change in $y$ to the change in $x$ ? [Short pause. No students respond.] If it's a linear function.

[Longer pause. No students respond.]

T: $\quad$ If we're graphing, which way do we go with $y$ ?

Coach: You might want to do a turn and talk right there. Because, otherwise, you're going to start to do that funneling question thing. Because I think I know what 
you're getting at. You have a particular word in mind, right?

T: $\quad$ Yeah.

Coach: Okay. So don't funnel us. Just say, 'Turn and talk. What does that mean? Nobody mentioned a ratio before. What does that mean in the definition?' Let us talk about it and then get it from us.

T: $\quad$ Okay. Turn and talk to your partner.

T: $\quad$ Okay, do I have any brave takers? What did we talk about?

Bridget: Earlier, we had the question about [Card] D. When she was explaining [Card] A, I connected it with [Card] D because the change in $y$ went up 6 in the first two points and $x$ went up by 1, so it would be 6 to 1 .

T: $\quad$ Which one are you talking about?

Bridget: [Card] D. So then in the next one, with $(-1,-1)$ and $(1,11)$, it went up 12 and the other side it went up 2 , so it was a 6 to 1 ratio.

Charity never managed to elicit the particular word she had in mind. Instead, Bridget described her own understanding of the ratio idea using one of the cards that had been sorted; the suggestion to give students a moment to think converted what might have been an unresponsive exchange into a responsive one. In her video annotation, Charity indicated awareness of her tendency toward funneling: "I fall victim to the funneling questions quite often if I don't get a response." She continued to be mindful of this tendency as the semester progressed. In her video annotation for the subsequent IA, WODB, she wrote, “...I feel like that in order to save time, I used a funneling technique to get students to help me graph the systems.” The coaching 
interjection around the funneling technique seemed to raise Charity's awareness for future rehearsals.

Although TCs did sometimes decide on their own to prompt students to take think time, instructional coaching helped TCs attend to student thinking by identifying moments when it was particularly useful to enact that move. Coaching introduced think time as a more productive way to elicit answers from students than, for example, asking a series of funneling questions, which brings the focus of the discussion to the teacher's ideas, rather than the students' ideas. There were fewer instances of $C$ Asks $T$ to Give Think Time as the semester progressed, which I attribute to TCs' growing ability to identify salient moments in which to enact the move. While TCs did enact T Prompts $S$ to Take Think Time (a move that was new to them) on their own more often as the semester progressed, they required more sustained support from me (the coach) to enact a different new move, Ask Different $S$ to Revoice, on their own. In the section that follows, I discuss interjections labeled C Asks T to Let S Revoice and how they compared to TCs' enactments of Ask Different S to Revoice.

C Asks T to Let S Revoice. As discussed in response to RQ1, asking a different student to revoice appears to have been a new move for TCs, as none of them enacted this move during their Teaching Demos (see Table 12). During their IA rehearsals in methods, however, TCs did enact this move, both with and without prompting from me. Of the 22 instances when Ask Different $S$ to Revoice was enacted by TCs during methods, seven could reasonably be attributed to coaching. Three interjections occurred during the first two IAs (GOP and CthenC), while the other four instances occurred during the final IA in methods (WODB). The increased emphasis on this move during WODB may have partially resulted from my own attention to the move throughout the semester, as described in response to RQ1. 
C Asks $T$ to Let $S$ Revoice. As noted above, I (the TE) gave more attention to the move Ask Different $S$ to Revoice at the start of the semester and again following the Card Sort rehearsals. Table 24 displays the number of responsive enactments of Ask Different $S$ to Revoice compared with the number of instances of $C$ Asks $T$ to Let $S$ Revoice. 
Table 24

Ask Different S to Revoice (responsive instances only) vs. C Asks T to Let S Revoice

\begin{tabular}{|c|c|c|c|c|c|c|c|c|c|c|}
\hline \multirow[b]{2}{*}{ Demo/IA } & \multicolumn{2}{|c|}{ Julian } & \multicolumn{2}{|c|}{ Bridget } & \multicolumn{2}{|c|}{ Charity } & \multicolumn{2}{|c|}{ Grace } & \multicolumn{2}{|c|}{ Totals } \\
\hline & $\mathrm{T}$ & Coach & $\mathrm{T}$ & Coach & $\mathrm{T}$ & Coach & $\mathrm{T}$ & Coach & $\mathrm{T}$ & Coach \\
\hline Teaching Demo $^{\mathrm{a}}$ & -- & -- & -- & -- & -- & -- & -- & -- & 0 & 0 \\
\hline GOP & -- & -- & 2 & -- & 1 & 1 & 1 & -- & 4 & 1 \\
\hline CthenC & 2 & 1 & 1 & -- & 1 & 1 & 2 & -- & 6 & 2 \\
\hline Card Sort & -- & -- & -- & -- & 1 & -- & -- & -- & 1 & 0 \\
\hline WODB & -- & 2 & -- & -- & 2 & -- & 2 & 1 & 4 & 3 \\
\hline Totals & 2 & 3 & 3 & 0 & 5 & 2 & 5 & 1 & 15 & 6 \\
\hline
\end{tabular}

${ }^{a}$ There were no coaching interjections at all during the Teaching Demos. 
I interjected six times throughout the semester to ask TCs to let a student revoice, with half of those interjections occurring during the WODB rehearsals. This is likely explained by my renewed attention to the move following the Card Sort rehearsals (as described in response to RQ1), rather than a regression in TCs' abilities to enact the move on their own. In each of these six instances, the TC immediately followed my interjection by enacting the move as suggested.

In two other instances, it seems reasonable to conclude that the TC asked a different student to revoice because of a recent (but not directly related) coaching interjection. Consider two consecutive S-T instances from Grace's WODB rehearsal. In the first, Grace asks Julian to revoice Bridget's idea at my (the coach's) request:

T: Were there any other reasons that your group discussed about either of these?

Bridget: We didn't discuss it, but just thinking about it, that one was probably, the $y=\sqrt{x}$ is probably the only one that has a possibility, it could have an imaginary solution. So if $x$ were to be like, negative something, it could have [inaudible].

T: $\quad$ So there's a possibility of an imaginary value there. Do you both have any other reasons for this card?

[No response.]

Coach: With the imaginary value, you might ask some of the rest of us to explain that a little bit more, because that's a newer idea than we've been talking about and it might be helpful to make it clear how we would get an imaginary value. That could be helpful for your discussion later on to talk about when that would happen. 
T: Okay, Julian, will you kind of restate what Bridget was saying about the imaginary value?

Julian: I think she was saying that the $x$ has a possibility of being negative, so taking it to the $1 / 2$ would make some kind of an imaginary value.

T: $\quad$ So let's think about this graphically. What would this look like if it was an imaginary value? Take a minute and think about that to yourselves...

Notice that when I interjected to ask Grace to have someone revoice Bridget's idea, Grace immediately responded by asking Julian to restate it. Now consider the S-T instance that immediately followed. Here, Grace decided on her own to ask Bridget to revoice Stephanie's idea:

T: $\quad$ Okay, so what do we think? Stephanie, will you give me some of your thoughts?

Stephanie: To get an imaginary value, we have to put in a negative number for $x$, but that wouldn't show up on the graph at all then, so if we put in $x=-1$, we don't graph that imaginary value, so I would look at that and say that even though the $\sqrt{x}$ can be imaginary, we wouldn't put in negative values of $x$. We would only use positive values, or at least non-negative values, of $x$.

T: $\quad$ Okay, so Bridget, can you restate what she was saying about the imaginary values?

Bridget: So if it was just an equation, it's a possibility that we could have the $y$ be equal to an imaginary number, but when you graph it, we wouldn't represent the imaginary values. 
Since these enactments of Ask Different $S$ to Revoice occurred in such rapid succession and were focused on the same topic, it seems likely that Grace may have still been attending to my coaching interjection when she asked Bridget to revoice in the second instance.

In her video annotation, Grace identified these two S-T instances as one long challenging moment. She wrote, “...I had not thought of this point. A lot was going on in my mind and it was difficult for me to determine what to do on the spot." While I did not interject because it seemed like Grace was having difficulty in deciding what to do, it seems that my interjection did give her an idea of how to handle this kind of challenging moment, when an idea is presented that the teacher had not considered beforehand.

This excerpt from Grace's rehearsal also provides evidence to suggest that coaching interjections have an impact beyond the TC's initial response. For Grace, this is apparent in her decision to enact Ask Different S to Revoice in the S-T instance following my interjection. Charity also described remembering previous comments to help her make decisions about her teaching. In her video annotation for her CthenC rehearsal, she wrote, "The final part of this clip shows student reasoning (as I remembered your suggestion of having students restate :) ).” These examples further make a case for the rehearsal model of teacher education-TCs do remember and attend to coaching suggestions in subsequent situations.

The two types of coaching interjections discussed above, C Suggests T give Think Time and $C$ Asks T to Let S Revoice, both highlight the coach's role in helping TCs enact moves that may be new to them. In these cases, simply enacting the move was often enough to ensure a responsive teaching instance. By highlighting these opportunities to use these moves, TCs may begin to use the moves more frequently, while also more judiciously and effectively. Other moves, however, are more common in TCs' practice but require enactment in particular ways in 
order to be responsive. In the next sections, I discuss C Comments on T's Recording and C Asks $T$ to Further Question $S$. These two types of coaching interjections center on moves that TCs already seemed familiar with at the start of the semester. However, TCs required some guidance to learn to enact those moves responsively.

Attending to recording. Recording students' ideas on the board makes them public, which is a key aspect of responsive teaching. While it is important to ensure that what is recorded is mathematically correct, it is also important to ensure that what is recorded accurately represents what the student said, particularly since there is an expectation for students to engage with other students' ideas. When these two goals are in conflict (i.e., when what the student says is mathematically incorrect), the TC should prioritize recording the student's idea and then provide opportunities for students to revise their thinking through discussions with others, ending by correcting the error(s) on the board. Before concluding an IA, the TC should ensure that what remains on the board is mathematically correct. This may involve erasing and correcting mistakes or simply striking through incorrect answers.

Although TCs did not discuss recording during any of their interviews, they occasionally commented on recording in their video annotations, usually to identify moments when they had difficulty deciding what to write. For example, Charity wrote after her CthenC rehearsal: "I had a little bit of struggling in trying to follow [Bridget's] way of thinking and write it on the board in a way it made sense to the class." Grace commented on a similar difficulty after her Card Sort rehearsal, though her issue was not misunderstanding a student idea, but in how to write the idea in a way that would be meaningful to the class discussion:

I had a difficult time determining exactly what to write. I can't remember exactly what I wrote, but I knew I needed to condense what was said a little bit and I knew I wanted to 
write it more generally...I wanted to be sure the students knew exactly what the function would look like when rearranged.

These comments make apparent the difficulties that can arise as TCs attempt to record student ideas in responsive ways. However, the TCs in this study generally managed to be responsive in their recording throughout the semester. Table 25 displays the number of responsive and unresponsive instances of T Records, organized by TC and IA. 
Table 25

Responsive and Unresponsive Instances of T Records

\begin{tabular}{|c|c|c|c|c|c|c|c|c|c|c|}
\hline \multirow[b]{2}{*}{ Demo/IA } & \multicolumn{2}{|c|}{ Julian } & \multicolumn{2}{|c|}{ Bridget } & \multicolumn{2}{|c|}{ Charity } & \multicolumn{2}{|c|}{ Grace } & \multicolumn{2}{|c|}{ Totals } \\
\hline & Resp. & Unresp. & Resp. & Unresp. & Resp. & Unresp. & Resp. & Unresp. & Resp. & Unresp. \\
\hline Teaching Demo & 1 & -- & 5 & 2 & 4 & -- & 5 & -- & 15 & 2 \\
\hline GOP & 2 & 2 & 7 & 1 & 6 & -- & 5 & -- & 20 & 3 \\
\hline CthenC & 5 & -- & 8 & -- & 6 & -- & 8 & -- & 27 & 0 \\
\hline Card Sort & 13 & -- & 15 & -- & 14 & -- & 12 & -- & 54 & 0 \\
\hline WODB & 12 & -- & 17 & -- & 8 & -- & $9^{a}$ & $1^{a}$ & 46 & 1 \\
\hline Totals & 33 & 2 & 52 & 3 & 38 & 0 & 39 & 1 & 162 & 6 \\
\hline
\end{tabular}

${ }^{\mathrm{a} A}$ single S-T instance included a responsive and an unresponsive enactment of T Records. 
TCs recorded student ideas 167 times during methods, primarily in responsive ways.

Unresponsive recording occurred when the TC recorded their own ideas, rather than student ideas. Note that all except one unresponsive instances of T Records occurred during the Teaching Demos or the first IA.

Although TCs consistently recorded student ideas responsively, there were moments when coaching interjections helped to improve their recording. Table 26 compares responsive instances of T Records and instances of C Comments on T's Recording that were focused on responsive teaching. 
Table 26

T Records (responsive instances only) vs. C Comments on T's Recording (responsive instances only)

\begin{tabular}{|c|c|c|c|c|c|c|c|c|c|c|}
\hline \multirow[b]{2}{*}{ Demo/IA } & \multicolumn{2}{|c|}{ Julian } & \multicolumn{2}{|c|}{ Bridget } & \multicolumn{2}{|c|}{ Charity } & \multicolumn{2}{|c|}{ Grace } & \multicolumn{2}{|c|}{ Totals } \\
\hline & $\mathrm{T}$ & Coach & $\mathrm{T}$ & Coach & $\mathrm{T}$ & Coach & $\mathrm{T}$ & Coach & $\mathrm{T}$ & Coach \\
\hline Teaching Demo & 1 & -- & 5 & -- & 4 & -- & 5 & -- & 15 & 0 \\
\hline GOP & 2 & 2 & 7 & 3 & 6 & -- & 5 & 2 & 20 & 7 \\
\hline CthenC & 5 & 2 & 8 & -- & 6 & -- & 8 & -- & 27 & 2 \\
\hline Card Sort & 13 & -- & 15 & -- & 14 & 1 & 12 & -- & 54 & 1 \\
\hline WODB & 12 & 3 & 17 & 1 & 8 & 1 & 9 & -- & 48 & 5 \\
\hline Totals & 33 & 7 & 52 & 4 & 38 & 2 & 39 & 2 & 164 & 15 \\
\hline
\end{tabular}


I commented on TCs' recording a total of 21 times throughout the semester. Six instances were not focused on responsive teaching. In these instances, I interjected to make a correction that was not a misrepresentation of a student's idea, but instead, was simply a mathematical error made by the TC.

Fifteen of the 21 total instances of C Comments on T's Recording were focused on responsive teaching, with half of those instances occurring during the first IA. The very first interjection I made related to recording was a compliment to Grace for "writing exactly what [Charity] said" as she explained the steps she used to solve a system of equations. (This instance was also labeled $C$ Compliments $T$.) This early interjection likely drew TCs' attention to careful recording, as evidenced in both their planning notes (see Figures 11 and 12) and their rehearsals throughout the semester. This kind of positive feedback is a useful tool for coaches and important to TCs' development as responsive teachers, as it highlights a successful enactment of a responsive teaching move for all TCs.

The instances of C Comments on T's Recording that were focused on responsive teaching took several forms. In several instances, I helped TCs identify and correct minor errors in their recording (e.g., "I think she said she subtracted 12 from both sides, right?”). In other instances, I encouraged the TCs to listen carefully to what the student was saying. For example, consider the following excerpt from Julian's GOP rehearsal:

T: $\quad$ So, Grace...show me the steps that you took to solve this.

Grace: $\quad$ So, I distributed and I had $-\frac{2}{3} r$.

T: $\quad$ Okay, so you multiplied this first, and then multiplied this by the $3 \ldots$

Coach: Don't put any words in her mouth. So, when you're recording, make sure you just record exactly what she says. 
In this example, Julian elicited a single step of the solution from Grace, but then continued solving the equation on his own. My interjection was a reminder that he should be listening to how Grace described the solution. Sometimes I suggested writing additional details on the board, as a means of keeping a careful record of the class discussion (e.g., "Why don't you write up there that $c=4 \ldots$ just so we can keep track of everything that's going on.").

In one unique instance, I suggested that Charity record an idea from Bridget, rather than just listening to it. In this excerpt from Charity's Card Sort, Charity had incorrectly sorted Card D in her planning, while the students had correctly sorted Card D during the IA, though Charity had not yet fully agreed with the students' conclusion. At this point in the IA, Charity had just given students think time to consider what the phrase "the ratio of the change in $y$ to the change in $x "$ means.

T: $\quad$ Okay, do I have any brave takers? What did we talk about?

Bridget: Earlier, we had the question about [Card] D. When she was explaining

[Card] A, I connected it with [Card] D because the change in $y$ went up 6 in the first two points and $x$ went up by 1 , so it would be 6 to 1 .

T: Which one are you talking about?

Bridget: [Card] D. So then in the next one, with $(-1,-1)$ and $(1,11)$, it went up 12 and the other side it went up 2, so it would be a 6 to 1 ratio.

Coach: This might be a time, Charity, when you want to record a little bit of what she's saying, even if you just mark those differences...just to track it a little bit.

Charity: All right. Do you mind restarting for me?

Bridget: [repeats her idea as Charity records] 
In this instance, as Charity worked to determine whether she or the students had made an error in sorting Card D, she did not think to record Bridget's idea. Charity wrote about this moment in her video annotation, "I marked this as a challenging moment because I was a little confused about what Bridget had said. I guess I was still trying to figure out if I had even put D in the right category.” I responded to this comment (in Vosaic Connect), “That's why I suggested that you write down what she was saying - both for the other students AND for yourself, to help you decide whether you agreed with Bridget's reasoning or if you thought she was incorrect."

Although some of the reasons for interjecting about TCs' recording were more substantive than others, each increased the responsiveness of the exchange taking place. TCs seemed to be learning from my interjections, as comments related to recording were made less frequently during successive IAs. This remained true until the final IA. It was during WODB rehearsals that we, as a class, began attending more closely to the balance between accurate recording and the pacing of the IA.

During WODB, I commented to Bridget (the first TC to rehearse WODB) that while it is important to record everything a student is saying, it is also important to be mindful of time and pacing:

T: $\quad$ Charity, which one did you decide did not belong?

Charity: The top right one.

T: $\quad$ Why?

Charity: It's the only one that stays in the top two quadrants, it crosses the y-axis at a positive interval. [long pause as T records]

T: $\quad$ And what was the second thing you said? 
Charity: It has a positive $y$-intercept.

T: [records 'only one to have positive y-intercept']

Coach: In recording, since we're going to be highlighting things that are unique about each tile, you don't have to write all of that. You can just put 'positive $y$ intercept.'...just to streamline it a little bit.

My suggestion that Bridget work to paraphrase the student's idea, while still remaining true to the student's meaning, seemed to create some confusion. In subsequent rehearsals of WODB, TCs began to paraphrase student ideas in ways that did not fully represent the student's meaning. Consider this example from later in Bridget's WODB rehearsal:

Grace: We said that it only crosses on the negative $x$-axis, it's not touching.

T: $\quad$ Okay, so we'll just exclude the origin then, so we'll say it crosses at 0 or less. Is that about the same thing as what you were trying to say?

Coach: Let's also say it crosses the $x$-axis at 0 or less, so that it clear which axis it's crossing.

In this instance, although Bridget was attending to an important mathematical detail (i.e., clarifying that the origin is not included as part of the negative $x$-axis), she did not record which axis Grace was talking about. This was an important detail to record because students were also noting differences in where the graphs crossed the $y$-axis. It seems that as Bridget was working to address my earlier comment about the pacing of the IA she overcompensated slightly by leaving out an important detail of Grace's response. This example from Bridget highlights the way in which this move was used with more complexity, which is a sign of development and demonstrates a need for continued growth; TCs were learning to balance careful recording with 
proper pacing of the IA. Thus, it was important to consider not only the number of times I interjected about TCs' recording, but also the nature of the C-T exchanges.

My analyses suggest that TCs attended more to recording as the semester progressed. They needed less correcting from me and fewer reminders to record students' ideas (not their own) over time. This is an important responsive teaching move for TCs to develop because proper recording is necessary for TCs to make student ideas public and to then engage students in mathematical discussions around those ideas. TCs must also understand, however, that there should be a balance between being careful with recording and being mindful of the timing and pacing of the activity.

Recording students' ideas seemed to be a teaching move that TCs expected to enact from the start of the semester. Initially, they required some support from me (the coach) to ensure that the ideas being recorded were mathematically correct and that they accurately represented the ideas shared by the student. As the semester progressed, my comments shifted to focus on more nuanced aspects of recording (i.e., properly recording student ideas while also attending to the pacing of the IA). This shift in my comments highlights TCs' development in recording student ideas, as they were eventually able to attend to more nuanced aspects of recording toward the end of the semester. TCs also attended to more nuanced aspects of a different teaching move, T Further Questions $S$, as the semester progressed.

T Further Questions $S$. Responsive teaching involves knowing when to probe students' ideas for further clarification or explanation. Because of their inexperience, TCs may not recognize moments when it would be particularly useful, or perhaps necessary, to ask a student to further explain what is meant by a mathematical term or description. However, the TCs in this study did recognize some opportunities to further question students on their own throughout the 
semester, though they also benefited from some coaching in this area. I first provide an overview of TCs' enactments of $T$ Further Questions $S$ and the types of questions TCs asked. In the remainder of the section, I will discuss my coaching interjections and how they impacted TCs' further questioning of students.

Reviewing instances of T Further Questions $S$ revealed that TCs asked different types of questions in their enactments of this move. I used a framework from Boaler and Brodie (2004) to identify the question types. Table 27 displays the number of both responsive and unresponsive instances of each question type as they occurred in methods. The instances in Table 27 do not include C-T instances. 
Table 27

Types of Questions Used in Enactments of T Further Questions S (adapted from Boaler and Brodie, 2004)

\begin{tabular}{|c|c|c|c|}
\hline Question Type & Description & Example & $\begin{array}{l}\text { Number of } \\
\text { Instances }\end{array}$ \\
\hline Probing & $\begin{array}{l}\text { Students are asked to further explain } \\
\text { their ideas }\end{array}$ & $\begin{array}{l}\text { T: ...it's not as steep as the other ones? } \\
\text { S: Right. } \\
\text { T: ...How do you know that it's not as steep } \\
\text { as the other equations? }\end{array}$ & 33 \\
\hline Gathering Information & $\begin{array}{l}\text { Require immediate answers; } \\
\text { Rehearses known facts/procedures }\end{array}$ & $\begin{array}{l}S: \text { Then I multiplied by the reciprocal. } \\
T: \text { And what is [the reciprocal]? }\end{array}$ & $13^{\mathrm{a}}$ \\
\hline $\begin{array}{l}\text { Exploring Mathematical } \\
\text { Meanings/Relationships }\end{array}$ & $\begin{array}{l}\text { Point to underlying mathematical } \\
\text { relationships and meanings }\end{array}$ & $\begin{array}{l}\text { S: There were steady increases. } \\
T: \text { What do you mean by steady increase? }\end{array}$ & $8^{\mathrm{a}}$ \\
\hline $\begin{array}{l}\text { Inserting } \\
\text { Terminology/Notation }\end{array}$ & $\begin{array}{l}\text { Teacher ensures that correct } \\
\text { mathematical language and/or notation } \\
\text { are used to discuss an idea that has } \\
\text { already been brought forward }\end{array}$ & $\begin{array}{l}\text { T: When we do systems, is there another way } \\
\text { we could write this? } \\
\text { Charity: An ordered pair. } \\
\text { T: So what would that be? } \\
\text { Charity: }(4,-4)\end{array}$ & 1 \\
\hline Total & & & 54 \\
\hline
\end{tabular}

ancludes one instance that is also counted in Probing 
Different types of questions elicited different levels of student reasoning. For example, Inserting Terminology/Notation or Gathering Information questions elicited responses from students that were easily evaluated as correct or incorrect. Consider this example of a Gathering Information question from Bridget's Card Sort rehearsal:

Grace: When you graph it, it's still a straight line and it passes the vertical line test, so it's a function.

$\mathrm{T}: \quad \quad$...and what is the vertical line test again?

Grace: [holds up her pencil vertically] So, the vertical line test is when you take your pencil and you move it across the graph and it only touches the function at one point.

Grace used the term "vertical line test" in her answer and Bridget asked her to explain it. If Grace had provided an incorrect definition, Bridget would have eventually needed to correct Grace's thinking. As a result, this was considered unresponsive because the focus was not necessarily on Grace's view of the problem, but instead, on a mathematical definition.

Alternatively, the two remaining question types, Exploring Mathematical Meanings/Relationships and Probing, elicited ideas from students that may have represented one of several possible correct perspectives from which to consider a problem or prompt. Consider this example of a Probing question from Bridget's CthenC rehearsal:

Grace: $\quad$... paying attention to the values of the numbers is important because then you can use rounding.

T: $\quad$ Can you explain a little more what you mean by 'values of the numbers', just to clarify? 
Grace: $\quad$ Sure. So I meant that if the value is, just like the rules of rounding, so if the value is higher you can round it up.

T: $\quad$ So, like in this case, it was 31 is close to 30 ?

Grace: Yeah.

In this instance, while Bridget has asked Grace to clarify a specific mathematical idea, there was more room for Grace's interpretation to be heard and accepted- “values of the numbers" was a phrase that Grace used in a vague way, which made it important for Bridget to push her to clarify what she meant by it. Because of this focus on Grace's thinking, the instance was considered responsive.

It could be argued that all 54 instances of $T$ Further Questions $S$ were responsive; by its very nature, this move elicits a student response, thereby making student thinking the subject of class discussion. For the purpose of considering the effect that coaching had on TCs' further questioning of students, however, I considered questions which had a definitive correct answer to be unresponsive. While these kinds of questions elicited responses from students, there was little room for students to provide their own perspective; instead, TCs asked them to define a term or identify proper notation, which is an important part of teaching, but not necessarily responsive. As a result, all thirteen instances of Gathering Information and the single instance of Inserting Terminology/Notation were unresponsive because the responses were easily identifiable as correct or incorrect. The other two question types, Exploring Mathematical Meanings/Relationships and Probing, were primarily used in responsive ways. Table 28 displays the number of responsive and unresponsive instances of Probing and Exploring Mathematical Meanings/Relationships questions, organized by IA. 
Table 28

Responsive and Unresponsive Instances of Probing and Exploring Mathematical

Meanings/Relationships Questions (coaching not included)

\begin{tabular}{cccccc}
\hline & \multicolumn{2}{c}{ Probing } & & \multicolumn{2}{c}{ Exploring } \\
\cline { 2 - 3 } \cline { 5 - 6 } Demo/IA & Resp. & Unresp. & & Resp. & Unresp. \\
\hline Teaching Demo & -- & -- & & -- & -- \\
GOP & 4 & 1 & & -- & 1 \\
CthenC & 2 & 2 & & -- & -- \\
Card Sort & 4 & 2 & & 2 & -- \\
WODB & 15 & 3 & & 3 & 2 \\
Totals & 25 & 8 & 5 & 3 \\
\hline
\end{tabular}

There was a marked increase in the number of probing questions asked during WODB, which I attribute in part to the structure of the IA. As students compared different representations of mathematical objects (e.g., equations, graphs), their ideas provided more opportunities for probing than the ideas put forward during the other IAs. Table 29 displays the number of responsive and unresponsive instances of each question type, organized by TC. 
Table 29

TCS' Responsive and Unresponsive Enactments of T Further Questions S (organized by question type)

\begin{tabular}{|c|c|c|c|c|c|c|c|c|c|c|}
\hline \multirow[b]{2}{*}{ Question Type } & \multicolumn{2}{|c|}{ Julian } & \multicolumn{2}{|c|}{ Bridget } & \multicolumn{2}{|c|}{ Charity } & \multicolumn{2}{|c|}{ Grace } & \multicolumn{2}{|c|}{ Totals } \\
\hline & Resp. & Unresp. & Resp. & Unresp. & Resp. & Unresp. & Resp. & Unresp. & Resp. & Unresp. \\
\hline Probing & 5 & 3 & 3 & 1 & 8 & 2 & 7 & 2 & 23 & 8 \\
\hline Exploring & -- & 1 & 4 & -- & 1 & -- & -- & 2 & 5 & 3 \\
\hline
\end{tabular}


Notice that each TC attempted and responsively enacted Probing questions more than any other question type. Bridget, Charity, and Grace each further questioned students responsively slightly more than half of the time, which may have partially been a result of coaching. In the remainder of this section, I discuss C-T exchanges labeled C Asks T to Further Question S.

Throughout the semester, I asked the TCs to further question students seven times; all of these interjections were focused on either Probing or Exploring questions. Table 30 compares the number of responsive enactments of $T$ Further Questions $S$ and the number of interjections labeled C Asks T to Further Question S. 
Table 30

T Further Questions $S$ (responsive only) vs. C Asks T to Further Question S

\begin{tabular}{|c|c|c|c|c|c|c|c|c|c|c|}
\hline \multirow[b]{2}{*}{ Demo/IA } & \multicolumn{2}{|c|}{ Julian } & \multicolumn{2}{|c|}{ Bridget } & \multicolumn{2}{|c|}{ Charity } & \multicolumn{2}{|c|}{ Grace } & \multicolumn{2}{|c|}{ Totals } \\
\hline & $\mathrm{T}$ & Coach & $\mathrm{T}$ & Coach & $\mathrm{T}$ & Coach & $\mathrm{T}$ & Coach & $\mathrm{T}$ & Coach \\
\hline \multicolumn{11}{|c|}{ Teaching Demo ${ }^{a}$} \\
\hline GOP & -- & -- & 1 & -- & -- & -- & 3 & -- & 4 & 0 \\
\hline CthenC & -- & 1 & 1 & -- & -- & 1 & 1 & -- & 2 & 2 \\
\hline Card Sort & -- & -- & 3 & 1 & 2 & -- & 1 & 1 & 6 & 2 \\
\hline WODB & 5 & 2 & 3 & -- & 7 & -- & 2 & 1 & 17 & 3 \\
\hline Totals & 5 & 3 & 8 & 1 & 9 & 1 & 7 & 2 & 29 & 7 \\
\hline
\end{tabular}

Note. Instances when the coach interjected are not counted in the T column. For example, during WODB, Julian further questioned students a total of seven times, with two of those instances being prompted by the coach.

aThere were no coaching interjections at all during the Teaching Demos. 
I made this type of interjection more frequently over time, which I attribute to the nature of the IAs, as opposed to a regression in TCs' development. Recall that the first IA, GOP, is concerned with solving a problem and the different strategies students used to obtain a solution. While some further questioning might be required during the class discussion of GOP, it is likely that these questions would focus more on terminology or procedures. Additionally, the questions to be asked are around things that might more obviously require clarification (i.e., using the correct notation in an answer). The remaining three IAs have at least some focus on listing observations about a problem or a series of mathematical representations (e.g., cards in Card Sort, or the four tiles in WODB). Eliciting observations from students provides more opportunities for students to introduce reasoning that require further clarification in the context of the IA, but this may occur in more subtle ways than during GOP. Thus, TCs may not always recognize which aspects of a student's idea to follow up on. In those instances, an interjection from the coach may help TCs decide what to follow up on, which in turn affects the type of question asked by the TC. Table 31 displays the number of instances of $C$ Asks $T$ to Further Question S, organized by TC and question type.

Table 31

C Asks T to Further Question S, organized by question type

\begin{tabular}{cccccc}
\hline & Julian & Bridget & Charity & Grace & Totals \\
\hline Probing & 2 & -- & 1 & 2 & 5 \\
Exploring & 1 & 1 & -- & -- & 2 \\
Totals & 3 & 1 & 1 & 2 & 7 \\
\hline
\end{tabular}


Interjecting to highlight underlying mathematics. The times when I interjected as the coach to ask the TCs to further question a student usually required listening for vaguely explained ideas in a student's response. Consider this excerpt from Julian's CthenC rehearsal, in which I suggest that he ask an Exploring Mathematical Meanings/Relationships question to give students the opportunity to articulate a mathematical principle. Here, Bridget has just described how she divided 0.9 by 5 mentally by solving an equivalent problem, 90 divided by 5 :

Coach: $\quad$...There's some things that Bridget did that we should just make explicit as to why you can do that.

T: Oh, okay.

Coach: She moved the decimal from 0.9, she turned that into 90, and then at the end, she had 18 , but she made it 0.18 . So...a question you can pose is, 'Why can we do that?' or 'Can we do that?' Was this coincidence or will this work all the time? So that's a question you want to pose that to the class and make sure everybody understands that.

T: $\quad$ Can somebody tell me why we can do this? Why we can move the decimal to make this 90, but we can move...the decimal [in 18] back?

Grace: $\quad$...I thought that it was okay to move the decimal as long as you remember to move it back at the end.

T: $\quad$ Yeah, yeah. So if you move the decimal in the beginning you have to make sure you do the same thing in your answer, right?

Grace: Yeah.

T: $\quad$ [to Bridget and Charity]...Did you have something different? 
Bridget: Well, you're doing inverse operations and that's why it works. Because, $0.9 \mathrm{x}$ $100=90$, but you can't leave it like that, and if you check $5 \times 18$ you're not going to get 0.9 . So you have to remember to divide...18 by 100 to get your 0.18 , so you're undoing operations.

Julian identified and commented on a longer version of this instance in his video annotation. His comment made it evident that he was very focused on the pacing of the IA, in addition to trying to understand Bridget's method: "That was tough, but in the end I understood what she was talking about. I really wanted to understand what she was talking about, but I probably took too much time to do this." His comment helps to explain why he may not have considered asking students to explain why the decimal could be moved back and forth — he was concentrating on other aspects of Bridget's idea. This demonstrates one affordance of the rehearsal model of teacher education - the instructional coach can bring up details that TCs might not notice.

Interjecting to help clarify student thinking. Sometimes, my interjections served a slightly different purpose. Instead of asking students to articulate an underlying mathematical principle, my interjections sometimes helped clarify vague aspects of a student's idea. Consider this excerpt from Charity's CthenC rehearsal, in which I ask Charity to use a Probing question to better understand Bridget's idea. The prompt asked students to order the fractions $\frac{4}{11}, \frac{7}{22}$, and $\frac{23}{66}$ from least to greatest:

T: Bridget, how did you approach this problem?

Bridget: I used two different ways to do it. I compared them all to a half and $\frac{4}{11}$ is the largest, so I had that out of the way. I knew it was greatest because it was 
closer to $\frac{1}{2}$ than the other two. So then I had $\frac{7}{22}$ and $\frac{23}{66}$ left over, but they were so close, where they would fall is so close to each other, that I just compared them to common denominators of 66 . And 21 is less than 23.

Coach: One thing that might be important to follow up on here is how she knew $\frac{4}{11}$ was closest to $\frac{1}{2}$, because she never really said that. Even though it's true, maybe ask her how she knew $\frac{4}{11}$ was closest to $\frac{1}{2}$.

T: $\quad$ So, how did you know that $\frac{4}{11}$ was closest to $\frac{1}{2}$ ?

Bridget: $\quad 5 \frac{1}{2}$ is half of 11 , so 4 is pretty close to $5 \frac{1}{2} .11$ is half of 22 , but 7 isn't that close to 11 . And 33 is half of 66 , but 23 isn't really that close to 33 . So, that's how I got the $\frac{4}{11}$.

I noticed an unexplained detail in Bridget's comment, (i.e., that $\frac{4}{11}$ is closest to $\frac{1}{2}$ ), and suggested that Charity probe Bridget's idea to help the class better understand her thinking. This kind of further questioning of students may require a more experienced ear, as evidenced by the fact that I made similar requests in four of the five coaching interjections about probing questions. Charity wrote about this interjection in her video annotation and explained that this was a challenging moment for her because she "had a little bit of struggling in trying to follow [Bridget's] way of thinking and write it on the board in a way it made sense to the class." In a comment specifically related to my coaching interjection, she acknowledged that she did not consider asking Bridget to further explain her strategy:

I hadn't even thought to make Bridget explain this idea to the rest of the class. Because I knew what she was doing (and obviously she did too), I just assumed the rest of the class 
would pick up on this. I'm sure I would've approached this a little differently if I was in an actual classroom and not a room of math majors.

TCs might also choose not to further question students in a methods class simply because they assume that all of the students already understand the mathematics. In a classroom full of mathematics education majors, it would be reasonable to expect that all students understood that inverse operations allowed for the movement of the decimal back and forth. There are two problems with such an assumption. First, even if the other TCs in the room understand this idea, actually articulating it may still prove to be difficult. Second, TCs will eventually be teaching in classrooms with students who do not already understand the mathematics content and who will require explicit explanations in order to gain an understanding of the mathematics behind a “trick." In order for TCs to successfully know when and how to further question secondary students in the classroom, they must practice those skills in the methods classroom - coaching interjections can help them identify those opportunities.

Coaching helped TCs' further question students responsively on their own. The data in Tables 28 and 30 provide evidence that TCs began to further question students on their own over time, particularly using Probing and Exploring Mathematical Meanings/Relationships questions. In doing this, they began to develop their abilities to follow up on more subtle aspects of student responses on their own as the semester progressed. Consider this example from Julian's WODB rehearsal:

T: Now, Stephanie and Grace, what is a reason that you guys had for picking this one $[y=x+7]$ as a non-example?

Grace: So, Stephanie said that it's not as steep as the other ones. 
T: Okay. What was the first thing that you said? That it was the only one with a slope of 1 ?

Grace: $\quad$ Mm-hmm.

T: $\quad$ [records] the only equation with a slope of 1

T: $\quad$ And then Stephanie said that it's not as steep as the other ones?

Grace: $\quad \mathrm{Mm}-\mathrm{hmm}$.

T: $\quad$ The graph isn't as steep?

Stephanie: Right.

T: $\quad$ Okay. [records] How do you know that it's not as steep as the other equations?

Coach: That was a really good question to ask me. That was a good follow up.

Stephanie: I know that a slope of 4 or a slope of 3 is going to make the line steeper, because for $4 x$ I would have to go up 4 and over 1, instead of just going up 1 and over 1 , so my graph is going to be steeper with $4 x$ and $3 x$. And then the third one with the $-2 x$, the line is still steeper than $x+7$, it's just going the other way. So I still would have to go up 2 and go to the left 1, but that's still going to be steeper than the $1 x$.

T: Okay, okay. So since we have this graphed right now, it looks something like this, so if it's steeper, then it would be somewhat like this. Is that what you're saying?

Stephanie: Right. Like for $4 x$ and $3 x$, but for $-2 x$, the line would be going the other way, T: $\quad$ So it would be going this way?

Stephanie: Right, but still changing more steeply than the $1 x$. 
T: $\quad$ Okay. That's a good point.

There are two things worth highlighting in this excerpt. First, notice that Julian asked the Probing question, "How do you know that it's not as steep as the other equations?" on his own, without prompting from me (the coach). This provides evidence of his development as a responsive teacher in terms of his skills in listening to student reasoning and then deciding when and how to probe those ideas. Second, this is an example of how $C$ Compliments $T$ might be seen during a rehearsal. By complimenting Julian, I highlighted his Probing question, "How do you know it isn't as steep as the other equations?" which then flagged the question (and similar questions) as a productive teaching move, not only for Julian, but also for the other TCs. This highlights the importance of the coach's providing positive as well as corrective feedback to TCs during their rehearsals.

Although TCs were further questioning students on their own from the start of the semester, there is evidence to suggest that instructional coaching helped TCs to refine their questioning skills. In particular, they began to listen for vague or incorrect statements in students' ideas. It is important for the coach to bring these ideas to the forefront, both to help the TCs recognize moments when further explanation is needed and, in some cases, to refine the TCs' own mathematical understandings. Compliments from the coach are helpful as well, in that they flag certain moves as productive in some way. All TCs participating in the rehearsal can learn from such compliments.

C Asks T to Ask Open Questions. One coaching interjection label that is related to the idea of questioning students is also worth mentioning here. Recall that in instances labeled $T$ Further Questions $S$, the TC required guidance from the coach to ask any question at all about the details of a student idea. In contrast, instances labeled $C$ asks $T$ to ask Open Questions refer 
to moments when the TC did pose a question, but in such a way that the desired response was obvious. This is not responsive teaching. Although the teacher might elicit the desired words or ideas from the students, by asking leading questions, the teacher is actually maintaining a focus on their ideas. It was these types of questions that led me to interject to ask TCs to ask more open questions. Of the seven instances of $C$ asks $T$ to ask Open Questions, six occurred during Bridget's rehearsals.

Consider this example from her GOP rehearsal. Bridget had elicited two possible solutions for solving an equation; both solutions were written side-by-side on the board:

T: $\quad$ So, have you noticed anything similar between your two methods? [pause] I see one step in particular that looks the same to me. [long pause]

Coach: $\quad$...as a student...I was ready to say something, but when you said 'there's one step in particular' I thought, 'Oh, I don't know which one she wants me to say.' That kind of stifled me a little bit. So, if you want to say that, just say, 'Do you notice any similarities?' Just leave it open, even though you have one in mind. You can keep that in mind and you can just make sure it comes out at some point, even if you have to be the one to say it...

Despite this early interjection reminding Bridget to phrase questions in such a way that allowed students to freely share their thinking (rather than verbalize what Bridget was thinking), she continued to struggle to ask questions in ways that would allow the students the freedom to share their reasoning. As a result, I made similar interjections during her rehearsals throughout the semester. By the end of the semester, however, she was showing progress in asking questions more openly. This will be discussed further in the next section, as I discuss moments when TCs 
paused their own rehearsals to ask questions of the coach. These questions from TCs tended to focus on TCs' further questioning of students.

Responding to TCs' questions in the moment. One significant affordance of the rehearsal model of teacher education is that TCs may choose to pause their own rehearsal to ask a question of the coach. Table 20 shows the number of instances of $T$ asks $C$ a Question, organized by substance. Table 32 displays the number of instances of $T$ asks $C$ a Question, organized by TC and IA. 
Table 32

Instances of T Asks C a Question

\begin{tabular}{|c|c|c|c|c|c|c|c|c|c|c|}
\hline \multirow[b]{2}{*}{ Demo/IA } & \multicolumn{2}{|c|}{ Julian } & \multicolumn{2}{|c|}{ Bridget } & \multicolumn{2}{|c|}{ Charity } & \multicolumn{2}{|c|}{ Grace } & \multicolumn{2}{|c|}{ Totals } \\
\hline & Resp. & Unresp. & Resp. & Unresp. & Resp. & Unresp. & Resp. & Unresp. & Resp. & Unresp. \\
\hline Teaching Demo & -- & -- & -- & -- & -- & -- & -- & -- & 0 & 0 \\
\hline GOP & -- & -- & 1 & -- & 1 & -- & -- & -- & 2 & 0 \\
\hline CthenC & -- & -- & -- & -- & -- & -- & -- & -- & 0 & 0 \\
\hline Card Sort & 1 & -- & -- & 1 & 1 & -- & 1 & 2 & 3 & 3 \\
\hline WODB & -- & -- & 1 & -- & 2 & 1 & -- & -- & 3 & 1 \\
\hline Totals & 1 & 0 & 2 & 1 & 4 & 1 & 1 & 2 & 8 & 4 \\
\hline
\end{tabular}


In the four unresponsive instances of $T$ asks $C$ a Question, TCs asked questions related to time constraints or mathematics content. Because of the straightforward nature of the responses to these questions, these instances will not be discussed further. Of the eight instances focused on responsive teaching, five were related to TCs' further questioning of students, which is a sign that this idea of questioning students was very salient to TCs. These five instances were labeled C Asks T to Further Question S, C asks T to ask Open Questions, or C Asks T to Highlight Math Idea.

In the three instances labeled $C$ Asks T to Further Question S, TCs wondered if they could be more direct in answering or posing questions. Consider the following excerpt from Grace's Card Sort rehearsal. As Grace walked around the room, Bridget and Charity were discussing the differences between irrational and real numbers during group think time:

Bridget: The real numbers include irrational numbers.

Charity: Do they? I don't remember.

Bridget: [to Charity] I think they do.

[to T] Can you tell us if real numbers include irrational numbers?

T: $\quad$ [out of screen and inaudible; seems to respond because Bridget and Grace appear to use her response to sort a card.]

Bridget: Okay, so that goes over there.

At the end of this rehearsal, I (the TE) quickly explained how the other cards should be correctly sorted. I also brought up the card $y=5 x^{4}-\pi x^{3}$ and the fact that it does represent a polynomial function. I mentioned that I overheard Bridget and Charity discussing irrational and real numbers earlier in the IA. It was at this point that Grace talked about what she was thinking in that moment: 
Grace: I Idn't know if I could tell her or not. I was kind of like, 'Eh, no.” But then it was just a coefficient, so I didn’t know.

Coach: When they ask you a question like that, yeah...

The fact that Grace was questioning how she should respond to Bridget shows that she recognized this as an opportunity to focus on student thinking. Although she was unsure about what her actions should be in the moment, the fact that she identified this opportunity as she was Teaching Demonstrates her development as a responsive teacher.

In the single instance labeled $C$ asks $T$ to ask Open Questions, Bridget enacted a similar instance of $T$ asks $C$ a Question during her WODB rehearsal. Recall that Bridget struggled all semester to avoid asking questions that would lead students to a particular phrase or idea; I interjected six times to request that she ask more open questions. During her WODB rehearsal, Bridget still seemed compelled to guide students with her questions, but this time, rather than asking the students a question, she first asked me what she should do:

T: [to the class] I want you to just think about this graph here to yourselves. Give me a thumbs up when you think of any reasons that it could be different. [pause] You can discuss with your partner.

T: [to Coach] So, if I have a group that isn't coming up with anything, can I give them a word to think about?

Coach: What do you mean, 'a word to think about?'

T: $\quad$ Like which way is it going, or something?

Coach: $\quad$ Maybe if the [inaudible] and then you can have [inaudible] because if they can come up with something, let them verbalize it. 
This example provides evidence that Bridget knew what not to do, but was still unsure of what she should do instead. Still, the fact that she paused to ask me (the coach) what to do, rather than automatically leading students with a "word to think about," demonstrates her growth as a responsive teacher.

In all of these instances, the hesitation TCs felt about taking the lead indicates growth in their understanding of responsive teaching practices; although their instincts were telling them to direct students' thinking by suggesting a word or idea, TCs also saw the need for asking questions more openly, to allow students the space to reason through the problem themselves.

In the previous two sections, I discussed two types of coaching interjections, $C$ Comments on T's Recording and C Asks T to Further Question S, both of which involved helping TCs enact familiar teaching moves in more responsive ways. As the semester progressed, TCs attended more carefully to recording student ideas accurately and efficiently, while also maintaining a reasonable pace for the IA and refining their questioning skills. In particular, instructional coaching helped TCs learn to ask more open questions and to identify vague or incorrect statements in student responses that may require further questioning. While all of the TCs responded favorably and productively to my coaching interjections, it is also important to consider how they viewed coaching and its impact on their teaching. In the final section of RQ2, I discuss TCs' perceptions of instructional coaching.

TCs' perceptions of instructional coaching. TCs' perceptions of instructional coaching are important to consider, as they may impact the extent to which TCs enact the coach's suggestions on their own, both during IA rehearsals and in school classrooms. Comments from TCs during their interviews and in their video annotations reveal that TCs viewed the coaching in different ways - some found my feedback helpful, while others, at times, seemed to view my 
feedback as critical. For the remainder of this section, I provide examples, in the TCs' own words, which illustrate their perceptions of my coaching.

TCs perceived coaching as helpful. In general, TCs' found instructional coaching to be helpful in their development as teachers. During both the mid- and post-interviews, I asked TCs, "Tell me what you think (how you feel) about the rehearsals/coaching. How are they affecting your development as a teacher?" Some TCs spoke more generally about the rehearsal process, while others talked more specifically about the coaching interjections, with some TCs identifying particular interjections they found helpful.

Bridget commented generally on the rehearsal process, without talking specifically about the coaching interjections. During her mid-interview, she said:

I like them. I feel like if we didn't do them ourselves, I probably wouldn't have given it as much thought to try to use it. And...actually doing something helps me to understand it a lot more. It's like more tools in your toolbox.

Julian and Grace both commented on the coaching aspect of the rehearsals more specifically. In his mid-interview, Julian said:

I like how you pause in the middle of what we're doing and you give that feedback...and then we know and can start over with it. It sticks in my mind better whenever you stop me right in the middle and tell me to do something different and then I can do it, instead of just waiting until after and I get the grade and then you write what I should have done. Julian highlighted the benefits of receiving feedback in real time. Grace made a similar comment during her mid-interview, though she also imagined times when coaching interjections could be more distracting than helpful: 
Grace: I think it's helping, because as I'm doing it, I'm able to think differently, especially if you say something, "well you could have done this," instead of me doing it and then later, you know, and then be like, "Well, okay, I don't even know my thought process when that was happening," you know? So I like that.

TE: $\quad$ So you think in the moment, giving you feedback right then is better than giving you the feedback after the fact. Is that what you mean?

Grace: Yeah. In a lot of cases, it's better.

TE: Are there any times when you feel like it's not better?

Grace: I don't think there has been. But I guess if I had a train of thought and then I lost it or something, because I got thinking about something that was said...that's all I can think of for that. If I was in front of actual students [while the coach was interjecting], that would be bad.

Grace also commented on the helpfulness of coaching during her post-interview, as well:

Grace: I thought they were beneficial, helpful...

TE: $\quad$...beneficial in what way? How were the rehearsals and the coaching beneficial, do you feel?

Grace: Because I was able to change and actually do what you were recommending me to do, instead of me thinking back and not really seeing how that would play out. Something about acting it out helps instead of just hearing about it afterwards. 
Both Julian and Grace appreciated receiving feedback in real time and then having the opportunity to implement that feedback right away; replaying those moments helped them to remember my suggestions more than only receiving feedback at the conclusion of the rehearsal.

In both their interviews and their video annotations, TCs also sometimes identified specific coaching interjections that they found particularly helpful. In her mid-interview, Charity talked about an interjection from her GOP rehearsal, when she was confused by something Julian said. After hearing his response, she said, "Okay. So, let's think this way..." and began to do a teacher-led example on the board. I (the coach) interjected to suggest that she ask another student to revoice what Julian said, rather than simply not responding to Julian. In her video annotation, she wrote about this instance:

This was a challenging moment because I was really confused by what Julian said and wasn't quite sure if I should ask him for more clarification. I knew he wasn't feeling well and wasn't fully with me so I wasn't quite sure if I should ask him more.

She further reflected on this moment during her mid-interview:

TE: $\quad$ Are there any particular coaching instances that stand out to you as being particularly either helpful or not helpful?

Charity: Probably the most helpful was when we did the GOP... [Julian] gave me that one response that I had no idea what he was meaning. I wasn't sure if I should ask him for more, ask someone else to attempt, because they all looked just as lost as I did...Bridget, for sure I could tell she was lost.

Charity's reflections on this difficult moment reveal that the coaching interjections can alleviate some of the stress the TC might feel in the moment by suggesting alternatives for continuing the IA and then giving the TC a chance to replay that moment immediately. 
Grace wrote about a moment when she answered her own question during her GOP rehearsal. In this instance, the coaching was not so much about navigating a difficult moment, but instead, about drawing attention to the fact that she had answered her own question and then offering an alternate course of action for similar moments that might occur in the future. In her video annotation, she commented:

This was a challenging moment. Right away I knew that the question I asked didn't make sense and wasn't what I meant to say. Next time, I plan to take more time to think about how to say the question I want to ask. If I ask a question like this again I plan to say, 'Let me rephrase that question,' so the students will be able to determine what I am asking and I won't have to answer it myself.

Grace also brought up this moment in her mid-interview:

TE: Do you have any particular instances of coaching that stood out to you...?

Grace: There was one when I said a question that was awkwardly stated, then I kind of had to answer it myself because they didn't understand what I was saying until after I answered it. And you said, "just take a second and think about it." TE: Oh, okay. Yeah.

Grace: So that was a good one because I wanted to be like, 'That didn't make sense.' With an instance like this, the interjection was less about helping Grace through a difficult moment and more about helping her to see a different way to handle similar situations in the future. During the rehearsal, I did not ask her to replay that moment — she had already answered the question. However, my interjection seemed to help her feel more comfortable about taking a moment to phrase the question mentally or, if necessary, to take a moment to rephrase a poorlyworded question, rather than answer it herself. 
Similarly, other TCs also made comments in their video annotations suggesting that my interjections gave them permission to ask questions, call on students, or provide think time when they either did not think to do those things at all or when they were unsure about whether taking such an action was appropriate. In the video annotation for her Card Sort, Charity wrote, "Thanks for jumping in and telling me that I can call on people based on what I see on their papers!" Grace also wrote about a moment during her Card Sort, when I (the coach) asked her to give students some think time: "In my head I was thinking to do a turn and talk, but I didn't listen to myself. Next time I will.” In this case, my comment seems to have reinforced an idea that Grace already had but did not follow up on.

Finally, sometimes TCs commented on the helpfulness of the coaching interjections as reminders of things they already knew they should be focusing on. In Julian's video annotation for his CthenC rehearsal, he wrote, "I don't know why I stopped and didn't put all she said. Good thing you [caught] that." In two of her video annotations Charity was appreciative of reminders not to assume that all students understand what is being discussed. In her CthenC video annotation, she wrote, "I had just assumed everyone understood Bridget's method. Thank you for pointing it out to me! I forget when doing these rehearsals that I'm practicing for a classroom, not solely teaching a room full of math majors." Then, a similar comment from her WODB video annotation:

In a room full of math majors (where I understand what we're all trying to say), it's easy to go along with any of their responses. In a room full of students, this can become difficult, as students need to use the mathematical precise language. Thanks for jumping in and clarifying this. 
In general, the TCs felt that participating in the rehearsals had a positive impact on their teaching. They especially appreciated receiving feedback in real time, having the opportunity to replay moments to act on my feedback immediately, and receiving help from the coach to navigate difficult moments. The fact that Charity, Grace, and Julian identified specific moments when the coaching had been helpful is encouraging; this provides evidence that they were internalizing what they were learning, which will hopefully have a positive impact on their responsive teaching in school classrooms. When TCs have a less than favorable perception of coaching, however, its ability to contribute meaningfully to TCs' development is diminished. In the next section, I consider evidence to suggest that one TC in this study may have seen some of my feedback as critical.

TCs perceived feedback as critical. Because of my position of authority, TCs may have been motivated to act upon my coaching interjections because those were actions I requested and, as the instructor of the course, I was responsible for evaluating their performance. When this occurs, TCs lack a true understanding of why I suggested certain moves at certain moments. This is problematic because it leaves TCs with a limited understanding of how and when to enact responsive teaching moves, making it less likely that they will enact those moves judiciously in classroom settings. In the current study, Julian is the only TC who provided evidence that this may have occurred. Although Julian responded well to my feedback during his rehearsals and directly commented on aspects of coaching he found to be helpful, other evidence from his interviews and video annotations suggest that he may have perceived some of my feedback as critical, rather than instructive.

Julian had a tendency to view his choices of teaching moves as right or wrong, with little room for negotiation. Our discussion of coaching during Julian's post-interview illustrates this 
point. I had chosen a specific coaching interjection to discuss with him because I wanted to talk about what he had planned to do if I had not interrupted him:

TE: $\quad$ There was one thing I wanted to look at here. [Takes a moment to load video.]

Julian: Did I do something bad?

TE: $\quad$ No. I just interrupted you during your lesson, and I wanted to ask you a question about it.

Julian: $\quad$ Oh, okay...

[We watch the video clip.]

TE: $\quad$...So right there, when I interrupted you, can you remember what you were thinking about when I stopped you? You had just asked Charity where the origin was and what she meant by that, and then I interrupted. Can you remember what you were thinking about?

Julian: I knew I was going to ask her what the origin was, and I did that. And then, I was thinking about if I was going to actually graph it or not. Because if I was going to graph that one, I would end up graphing the other three, and I didn't know if I would have time for it, or even if I should do it. So, yeah, I was kind of undecided on that one.

TE: $\quad$ Sure.

Julian: What should I have done?

TE: I'm not saying it's right or wrong. I was just curious, because I interrupted your train of thought and I didn't get to see what you would have done at that point. 
This excerpt illustrates Julian's high level of concern for doing things in ways that would meet my approval. This focus seemed to overshadow his ability to discern when to enact certain teaching moves, as evidenced in his WODB rehearsal—-he found a reason to further question almost every student response. This left me wondering if he really felt it was important to follow up at all of those times or if he thought that was what I wanted him to do, as a result of the comments I made to other TCs during their WODB rehearsals. Consider the following example from his WODB rehearsal:

T: $\quad$ Why did you pick it?

Stephanie: Because it has a negative slope?

T: How did you know that it's a negative slope?

Stephanie: Because the number in front of $\mathrm{x}$ is negative, and that represents the slope, and it's the only one that's negative.

In this second example from his WODB rehearsal, I (the coach) interjected to help Julian refocus his questions slightly, in order to better address the goals of the IA:

T: $\quad$ Did you have anything different for this card?

Charity: I said that if you graph it, it's the only one that will cross the origin.

T: Okay. So if we were going to graph it, well, where is the origin at?

Charity: Right there where they meet.

T: $\quad$ So it would be right here?

Charity: Mm-hmm.

T: $\quad$ So this would be the graph, it would go through this point right here?

Charity: Mm-hmm.

T: $\quad$ Okay. Were there any other reasons why you picked this card? 
Coach: ...You might also ask her how she knew it was going to go through the origin, because that's not clear from the equation. So, that's something else you might follow up on.

T: $\quad$ So how did you know that it would go through the origin?

Charity: Because if $\mathrm{I}$ add the zero on to it, that puts it in $\mathrm{y}=\mathrm{mx}+\mathrm{b}$ form, so zero would be the y-intercept.

T: $\quad$ Okay, that makes sense.

These examples represent only two of the ten times Julian further questioned students during his WODB rehearsal. It is important to note that the results of $T$ Further Questions $S$ in these instances were not necessarily unresponsive—-here, I am merely providing evidence to support the claim that Julian may have been using this move in a more habitual way, rather than carefully considering what he hoped to gain by further questioning students. The fact that, in the second excerpt above, I needed to interject to help Julian ask a question that was more focused on his goals also supports the idea that Julian may have been further questioning students because he thought it was what I wanted him to do, rather than attempting to use the move judiciously.

Julian's video annotation comments also suggest that he viewed my coaching interjections as disapproving. One of the video annotation prompts asked TCs to identify and comment on moments when they felt supported or when they felt they could have used additional support. Julian's comments on these moments seem to be more of a cataloging of the reasons I interjected - they read more like a list of "dos and don'ts" (see Figure 21): 


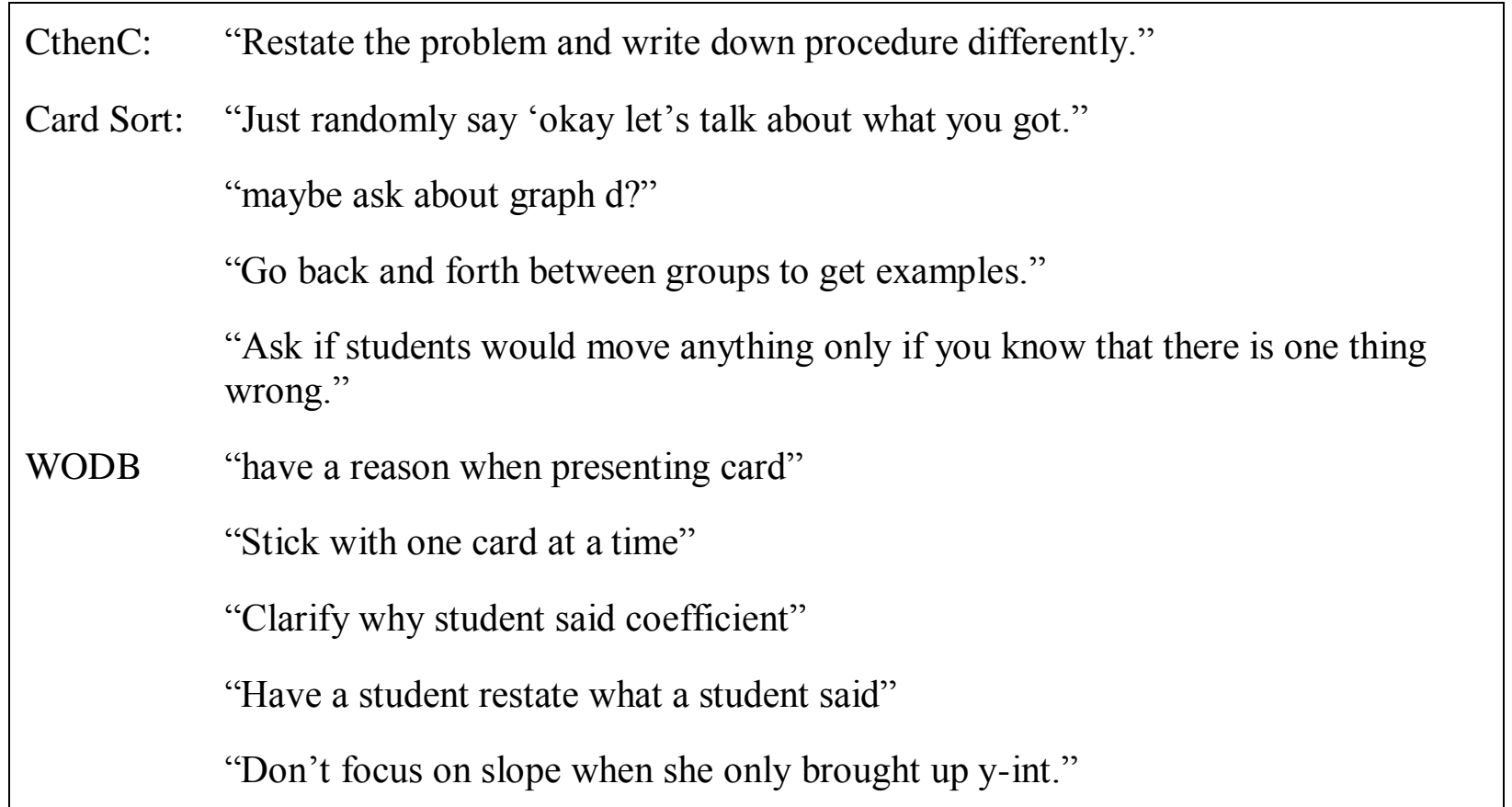

Figure 21. Sample of Julian's video annotation comments for moments he labeled Support Some of these comments are extremely specific to our context (e.g., "maybe ask about graph d?’), while others are written so generally that there is no evidence that Julian understood why I made a particular recommendation at that particular moment (e.g., "Have a student restate what a student said"). His focus on teaching in ways that would meet my approval seems to have hindered his ability to fully understand the purposes of teaching moves and how to leverage their use.

It is important to consider TCs' perceptions of coaching as a means of helping TCs maximize the benefits of this form of teacher education. Although coaching helped TCs develop skill in responsive teaching, both in enacting unfamiliar teaching moves ( $C$ Suggests that $T$ Provide Think Time and C Asks $T$ to Let S Revoice) and familiar teaching moves (C Comments on T's Recording and C Asks T to Further Question S), their perceptions of the purposes of my interjections may inhibit their judicious use of responsive teaching moves, both in methods and in school classrooms. In the next section, which focuses on analyses of TCs' enactment of the 
GOP IA in a school setting (without the presence of an instructional coach), I will examine TCs' responsive teaching and draw some conclusions about how the coached rehearsals in methods affected TCs' practice with secondary students.

\section{RQ3: To What Extent Do TCs Teach Responsively in School Settings?}

In addition to the four coached rehearsals in methods, TCs were also asked to enact the GOP IA in a classroom setting, which I will subsequently refer to as School GOP. One goal of this assignment was to encourage TCs to practice responsive teaching with secondary mathematics students. For the purposes of this study, however, I also wanted to see how TCs enacted moves differently (if at all) in a more authentic setting. Results indicate that, in school settings, TCs enacted responsive teaching to a greater extent than in their original Teaching Demos, but to a lesser extent than in their coached rehearsals. Thus, TCs ultimately developed into more responsive teachers over the course of the semester, despite the fact that some teaching moves were enacted differently in the school setting than in methods.

TCs' initial preparation for School GOP placed more responsibility on them in terms of choosing a problem and writing goals for the IA. For each of the four IAs in methods, I assigned the prompts and goals for each TC. Because the TCs had a better understanding of their school context than I did, TCs worked with their mentor teachers in the schools to choose an appropriate topic or perhaps even a specific problem, depending on the preference of the teacher. TCs were also responsible for writing appropriate goals and completing the planning materials. TCs' problems and goals for their School GOP enactments may be found in Appendix G.

In the sections that follow, I first provide descriptive data related to TCs' school enactments. I then provide four vignettes to give an overview of each TCs' preparation for and enactment of School GOP. I also provide a brief commentary for each TC to highlight 
particularly salient aspects of their school enactment as compared with their rehearsals in methods.

Descriptive data. A comparison of TCs' Teaching Demos and School GOP enactments reveal that, overall, TCs became more responsive in their teaching over the course of the semester. Compared to their Teaching Demos, TCs engaged in twice as many S-T instances per IA, with each instance during School GOP lasting an average of three times as long as each instance during the Teaching Demos (see Table 33). This suggests that the TCs led the IAs in schools in ways that were similar to their IAs in methods, by engaging student ideas more frequently and more deeply than in their Teaching Demos.

Table 33

Number and Length of S-T Instances per IA, Including School GOP

\begin{tabular}{ccccc}
\hline Demo/IA & $\begin{array}{c}\text { Average Length } \\
\text { of Demo/IA }\end{array}$ & $\begin{array}{c}\text { Total Number } \\
\text { of S-T } \\
\text { Instances }\end{array}$ & $\begin{array}{c}\text { Average } \\
\text { Number of S-T } \\
\text { Instances per } \\
\text { Demo/IA }\end{array}$ & $\begin{array}{c}\text { Average Length } \\
\text { of S-T Instances } \\
\text { per Demo/IA }\end{array}$ \\
\hline Teaching Demo & 4.5 min. & 27 & 6.75 & 13 sec. \\
GOP & 16.7 min. & 53 & 13.25 & 37 sec. \\
CthenC & 17.0 min. & 53 & 13.25 & 43 sec. \\
Card Sort & 29.2 min. & 64 & 16 & 55 sec. \\
WODB & 23.8 min. & 50 & 12.5 & 1 min. 10 sec. \\
School GOP & 15.6 min. & 48 & 12 & $41 \mathrm{sec}$. \\
\hline
\end{tabular}

${ }^{\mathrm{a}}$ Calculated using the Instance Frequency Report from Studiocode

Responsive teaching, however, is concerned with more than the amount of time TCs engage student ideas. Table 34 provides a more detailed overview of the teaching moves enacted 
by TCs during their School GOP enactments, as well as the number of times each move was used responsively and unresponsively. 
Table 34

TCs' Responsive and Unresponsive Use of Teaching Moves During School GOP

\begin{tabular}{|c|c|c|c|c|c|c|c|c|}
\hline \multirow[b]{2}{*}{ Teaching Move } & \multicolumn{2}{|c|}{ Julian } & \multicolumn{2}{|c|}{ Bridget } & \multicolumn{2}{|c|}{ Charity } & \multicolumn{2}{|c|}{ Grace } \\
\hline & Resp. & Unresp. & Resp. & Unresp. & Resp. & Unresp. & Resp. & Unresp. \\
\hline Ask Different $\mathrm{S}$ to Revoice & -- & -- & -- & -- & 5 & 3 & -- & 2 \\
\hline Ask $S$ if they Agree/Disagree & -- & -- & 2 & 2 & -- & 2 & 1 & 3 \\
\hline $\begin{array}{c}\text { T Prompts } \mathrm{S} \text { to Take Think } \\
\text { Time } \\
\text { (IA) }\end{array}$ & 1 & -- & 2 & -- & 2 & -- & 2 & -- \\
\hline $\begin{array}{c}\text { T Prompts } \mathrm{S} \text { to Take Think } \\
\text { Time } \\
\text { (not in IA protocol) }\end{array}$ & -- & -- & -- & -- & 1 & 2 & 5 & 2 \\
\hline T Further Questions S & -- & 2 & -- & 1 & 5 & 1 & -- & -- \\
\hline $\begin{array}{l}\text { T Invites Other Ideas from } \mathrm{S} \\
\text { (none elicited) }\end{array}$ & 1 & -- & -- & -- & 1 & 1 & -- & -- \\
\hline T Makes Connection & -- & -- & -- & -- & -- & -- & 1 & -- \\
\hline T Records & 6 & -- & 7 & -- & 5 & 2 & 7 & 3 \\
\hline T Repeats & -- & 7 & 2 & 4 & -- & 5 & 1 & -- \\
\hline T Revoices & -- & -- & 2 & -- & 2 & 1 & 1 & -- \\
\hline
\end{tabular}


The moves included in Table 34 are a subset of the moves that were enacted during TCs' rehearsals. Moves that were used very infrequently (or not at all) during School GOP were not included in the table. For example, some moves, such as T Confirms Recording and T Clarifies, were used during rehearsals but were not used in schools. Another move, T Corrects, first occurred during TCs' school enactments, but was used unresponsively only twice by a single TC.

Notice that TCs enacted several responsive teaching moves during their School GOP enactments that were not used during their Teaching Demos (e.g., T Prompts $S$ to Take Think Time, Ask a Different $S$ to Revoice). Although not every enactment of these new moves was responsive, the TCs' attempts to enact them in schools is indicative of their development as responsive teachers. TCs first learned about some of these moves during the methods course (e.g., Ask Different $S$ to Revoice); their efforts to enact those moves with secondary students is suggestive of their desire to engage student ideas. However, further insight into TCs' development may be gained by considering how they enacted these moves in schools.

I have chosen to organize my response to RQ3 in a series of vignettes (one for each TC) to present a more complete picture of each TC's development. In the vignettes, I discuss some teaching moves in more detail, as they relate to individual TCs. For each vignette, I first discuss the TC's planning work. Then, I describe their enactments to provide a sense of the atmosphere of each classroom and TCs' responsive teaching during the IA. Finally, I provide a commentary to highlight salient aspects of each TC's enactment that speak to their development as responsive teachers.

Julian. Julian enacted the School GOP IA in a sixth grade classroom, thanks to the cooperation of one of Julian's former mentor teachers. (Recall that Julian was the only TC not concurrently enrolled in the education course that required a 75-hour school placement.) His IA 
lasted approximately 8 minutes from start to finish, making it the shortest School GOP enactment. Approximately 11 students participated.

Planning materials. Julian let me know ahead of time what type of problem he would use for School GOP to determine whether his problem would work reasonably well for the IA. I agreed that the problem he had chosen had the potential to promote class discussion. Beyond that, I was not involved in the other aspects of his planning.

Reviewing Julian's planning materials revealed that he had some difficulty in writing and/or articulating the mathematical goals for the IA (see Figure 22).

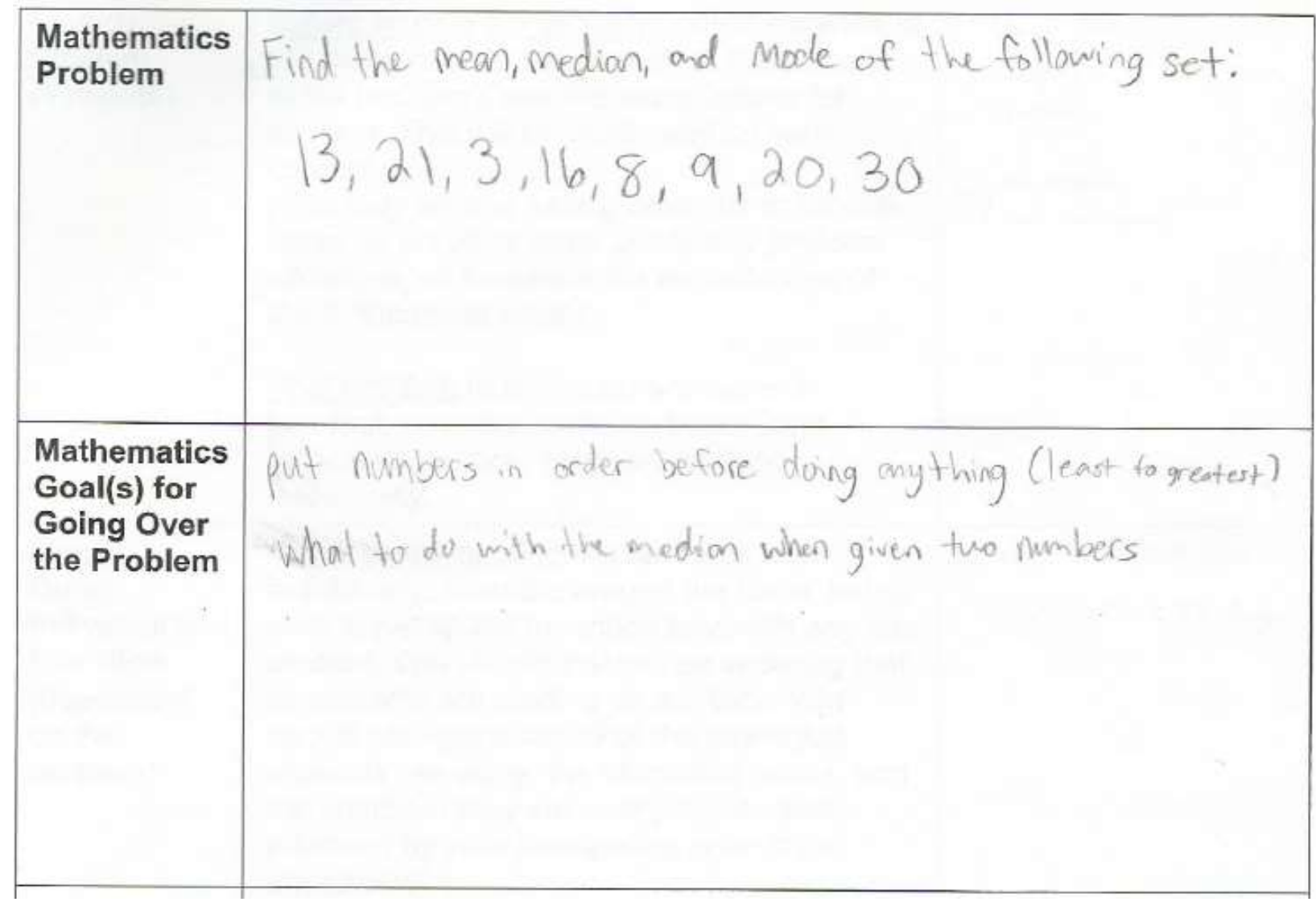

Figure 22. Julian's Problems and Goals for School GOP.

Notice that his problem asks students to "find the mean, median, and mode," but his mathematical goals for the IA are only related to finding the median. (To correctly find the mean 
and mode, the numbers are not required to be in numerical order.) His goals are also not stated in a way that makes it clear what the students should be able to do. For example, his second goal would be better stated as, "Students will articulate how to find the median of a data set when the set has an even number of values." Recall that Julian was not enrolled in the same education course as the other TCs and as a result, did not have the same level of instruction as the other TCs prior to completing this assignment. An examination of Julian's planning materials reveals that he would have benefited from additional support from me (the TE) to write goals that better aligned with his problem and that articulated what action should be expected from students. Some assistance in anticipating student reasoning might also have been helpful.

Preparing a thorough set of anticipated student responses ahead of time can help the TC gather ideas of strategies and mistakes to watch for as students are working and to make decisions about which strategies or mistakes to consider highlighting during the class discussion. Throughout the semester, Julian seemed to have difficulty in anticipating student reasoning. Recall that, in his mid-interview, Julian reported having difficulty anticipating student reasoning as he prepared for each IA. This seemed to have remained the case for his School GOP IA (see Figure 23). 


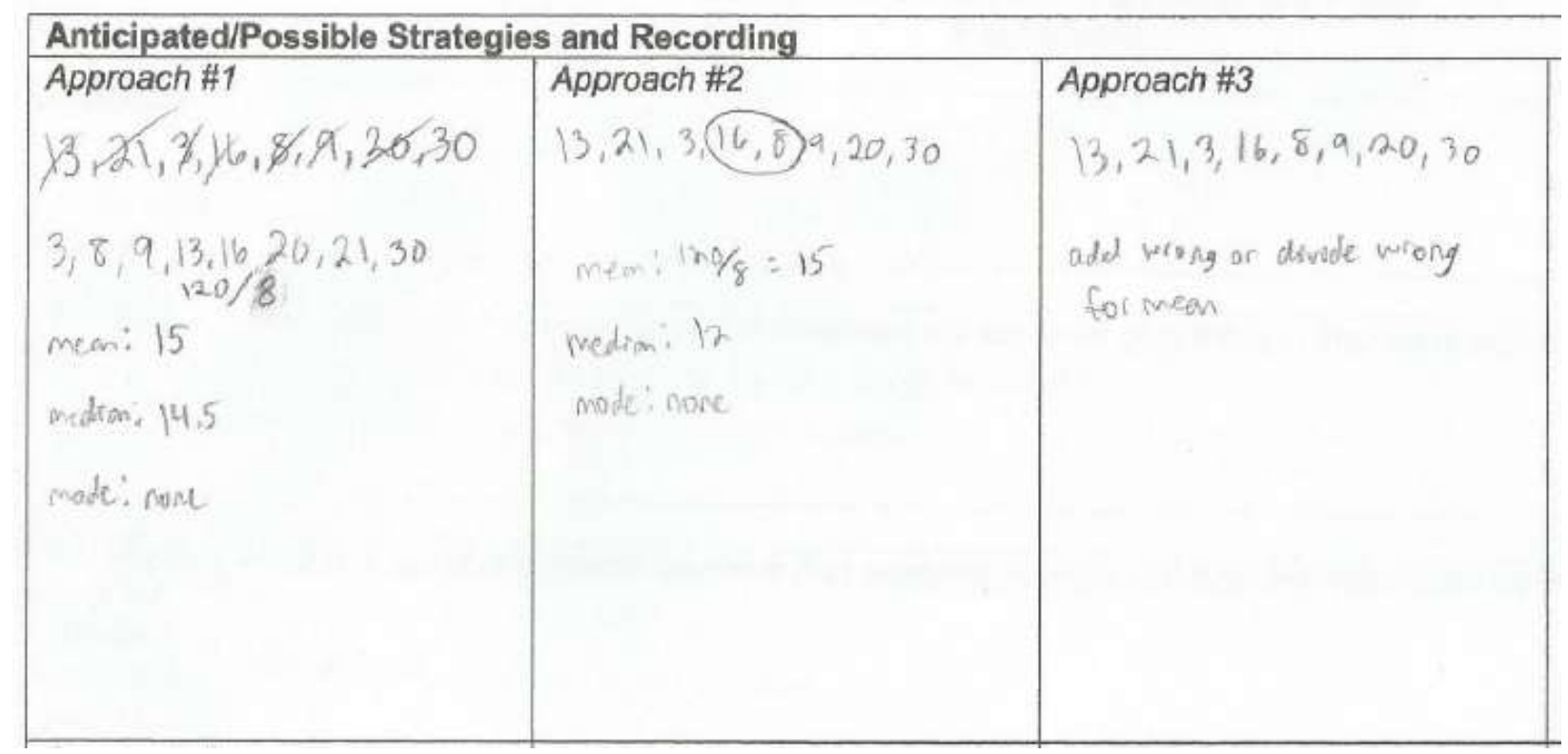

Figure 23. Julian's Anticipated Student Responses for School GOP

Julian's anticipated student responses include one that is completely correct, one that only has an incorrect answer for the median (with the error being that the numbers were not ordered before performing the calculation), and the third is a general note that students may make arithmetic errors in calculating the mean.

It is worth mentioning that, because Julian was not regularly working with students in classrooms throughout the semester, he may have been at a slight disadvantage in terms of anticipating student reasoning for his School GOP IA. The other three TCs had been working with the same groups of students and the same mentor teacher throughout the semester - they had likely learned from seeing the kinds of mistakes their students were prone to make. Julian did not have the same opportunities to learn from repeated work with the same group of students who participated in the IA.

Julian's protocol notes are also worthy of consideration. Similar to his planning notes for the IAs in methods, Julian's notes were vague; they did not include any specific teaching moves 
to enact or specific student ideas to listen for. His notes included reminders to "walk around and look to what students are doing" and "walk around and listen to groups (listen for things to discuss)." As an example of a more specific note, instead of writing "look to what students are doing," he might have written something like, "look for students who forget to put the numbers in order to find the median." More specific notes would have helped him to think more carefully about what he wanted to highlight in the class discussion and might have helped him get those ideas from students. Instead, Julian asked some leading questions to help him reach his mathematical goals during his enactment, which will be discussed in the next section.

Enactment. Julian began by posting the problem on the board. He read the problem aloud and asked the students to work individually to solve it. As the students worked, he circulated throughout the room and looked at students' papers. Once most students seemed to be finished working individually, Julian asked the students to work in groups of two or three to discuss their solutions. Again, Julian circulated throughout the room, pausing and looking toward students' papers as he moved from group to group.

He regained the students' attention for whole class discussion and then used a seating chart to call on particular students to share the answers they got for the mean. After each group responded, Julian asked if anyone else had a different answer; none of the students spoke up.

Next, he called on the group who had given their answer for the mean to explain how they arrived at that answer. The students responded, nearly in unison, that they had added the numbers and divided by eight. Julian then asked, "Did you have to do something first?" to which the students responded that they put the numbers in order first. Julian asked them which number would go first and the students proceeded to read their ordered list of numbers as he recorded them on the board. Julian then repeated the procedure as they had explained it: "So you said you 
added up all of these numbers and divided by 8 , and that's how you got your mean?' One member of the group nodded.

Julian then called on one specific student to explain how he found the median. The student responded that he had to find the middle two numbers. Julian asked, "What would be the middle two numbers?" Once the student responded, he circled those numbers on the board, then asked the student to continue with his explanation. The student explained that he found the number in between the two middle numbers:

Julian: You found the number that was in between them?

Student: You find the mean of those two numbers.

Julian: I like that. You find the mean of those two numbers. So you added these two numbers and then divided by what?

Student: Two.

Julian: $\quad$ Two. And then that's how you get 14.5.

Finally, Julian asked a third group how they found the mode. One student responded, "You look for the numbers that repeat." Julian affirmed that answer, then began to write out the steps required to find the mean, median, and mode. The following transcript is from the last moments of the IA:

Julian: Let's solve this out. What did you get whenever you added this set right here? What was the total number?

Students: 120 .

Julian: $\quad 120$ ? You got 120, then you divided it byStudents: 8 . 
Julian: $\quad$. And that's how you got 15 , which is the mean. And the median, we already discussed that, you find the two middle numbers, add them together, and then divide by two, so in this, you basically have to do the mean to find the median, and you get 14.5, and that's correct. And what did you guys say? How did you know that there was no mode?

Students: None of the numbers repeated.

Julian. Yeah. None of the numbers repeated. There are no repeating numbers in this, so there is no mode. Okay, we're good then. Thank you, guys.

Commentary. Julian showed some development in responsive teaching during his School GOP enactment, when compared with his Teaching Demo. Most notably, he gave students time to solve the problem individually and in groups before discussing the problem as a class. While this was a move that was suggested in the IA protocol, enacting it responsively is evidence of growth.

In other ways, however, his School GOP enactment closely resembled his Teaching Demo, in both responsive and unresponsive ways. During both enactments, Julian responsively recorded student ideas on the board by writing exactly what the students said. It is encouraging that his responsive enactment of $T$ Records did not regress during School GOP.

Another similarity between Julian's Teaching Demo and School GOP IA may be seen in his questioning strategies. During his Teaching Demo Julian asked only two questions, each of which had a single correct answer, causing them to be considered unresponsive. During his School GOP IA, he asked more questions, but they resulted in either leading students to an idea that Julian wanted to bring forward (e.g., "Did you have to do something first?") or they were 
questions that had easily identifiable correct answers (e.g., "What did you get when you added this set right here?"). These questions were considered unresponsive.

Throughout the entire semester, Julian habitually repeated student ideas unresponsively. He enacted the move $T$ Repeats more than any of the other TCs during his IA rehearsals in methods. This remained true during his school enactment; he repeated student ideas seven times, none of which were considered responsive (i.e., none of the instances seemed to be used to ensure proper recording). Here is a representative example of Julian's enactment of T Repeats:

Julian: What did you get for your median?

Student: 14.5 .

Julian: Okay. 14.5. Tiffany and Edward, what did you get for the mode? While not necessarily detrimental, Julian's repeating of the student's answer was also not responsive. As discussed in response to RQ1, unresponsive enactments of T Repeats are usually not problematic, though TCs should be aware of how often they enact the move, as overuse could become distracting for students, which could then detract from the overall responsiveness of S-T exchanges.

As the IA progressed, Julian's teaching became less responsive overall, with Julian doing much of the explaining. When he asked students about how they found the median, we see one missed opportunity for a responsive exchange:

Student: You find the mean of those two numbers.

Julian: I like that. You find the mean of those two numbers. So you added these two numbers and then divided by what?

After Julian repeated the student's response, he then made an assumption about how the student found the mean (i.e., by adding the numbers and dividing by something). This could have been 
more responsive if Julian would have said, "I like that. You find the mean of those two numbers. How did you do that?" In that case, the student would have been free to respond with the process he had used, which would have put the emphasis on student thinking.

Julian's school enactment displayed limited development as a responsive teacher, which highlights areas for continued improvement. During his School GOP enactment, he stumbled in many of the same ways as his IA rehearsals (e.g., asking questions that lead students to a particular response). His planning, particularly establishing goals, could have been impacted by the lack of teacher education experiences that would support those practices, which were not explicitly supported by the IA rehearsals. Other aspects of his enactment raise questions about the potential impact of his not knowing the students (and the students not knowing him) as a result of the lack of a concurrent school placement. These highlight implications that I discuss more in Chapter 5. While the other three TCs demonstrated more development of responsive teaching practices through their school enactments, they each present different cases that highlight different aspects of responsive teacher development. These are discussed in the vignettes that follow.

Bridget. Bridget enacted the School GOP IA in an eighth grade classroom. The video recording of her IA lasted approximately 11 minutes, but gaps in the recording suggest that the individual and group think time were not recorded in their entirety. Thus, while her recording was shorter than the average length of TCs' School GOP enactments, there is reason to believe that her enactment of the IA was slightly longer than 11 minutes. Approximately 18 students participated.

Planning materials. By chance, Bridget's problem for her School GOP IA closely resembled the problem I had assigned her for her GOP rehearsal. Recall that, in preparation for 
the rehearsals during methods, Bridget demonstrated a strong ability to anticipate a variety of possible student reasoning strategies for each prompt. This was true for her School GOP rehearsal as well—she identified a variety of student responses, including subtle variations of strategies (e.g., Approaches \#2 and \#6; see Figure 24).

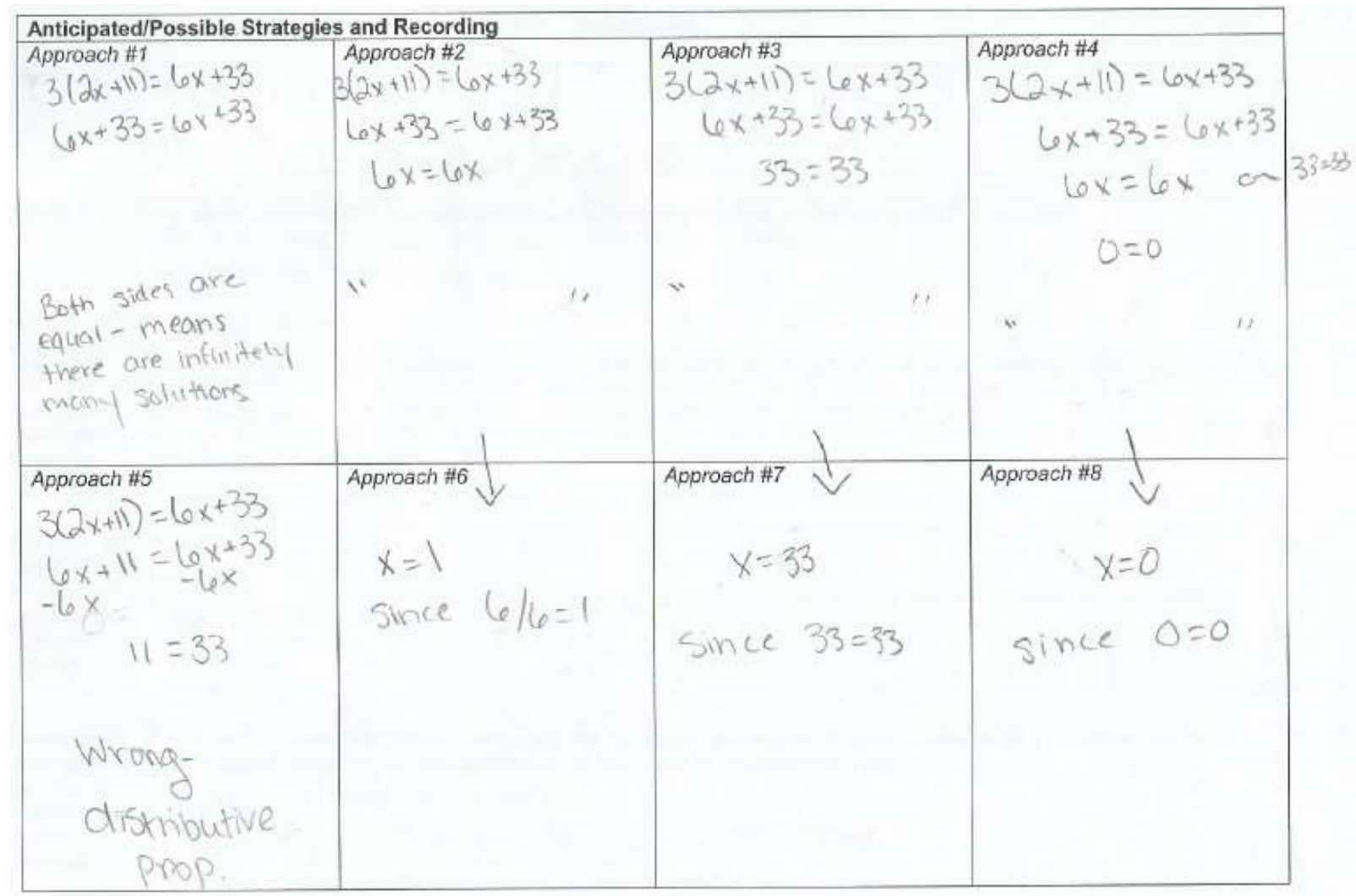

Figure 24. Bridget's Anticipated Strategies for School GOP.

Although Bridget's goals were consistent with her problem, they were not worded in ways to specifically describe what the students should be able to do. For example, one of Bridget's mathematical goals was, "distribute correctly" (see Figure 25). 


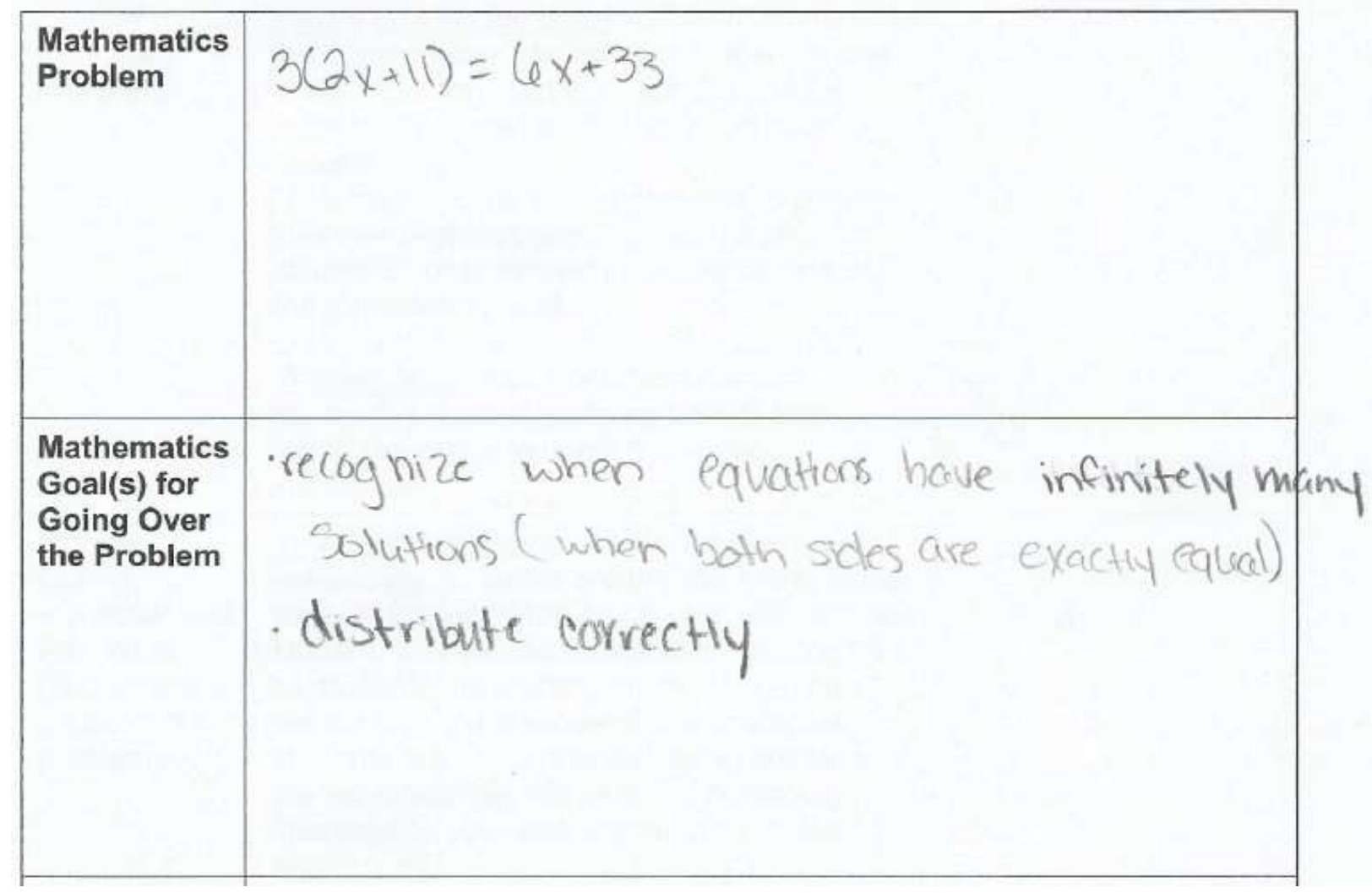

Figure 25. Bridget's Problem and Goals for School GOP.

Clearly articulated goals are important to maximize the potential of the IA to be responsive. For example, students in Bridget's class could successfully meet her goal of "distribute correctly" by individually solving the problem on paper. While this would provide evidence that students understood how to apply the distributive property (assuming that the teacher examined each student's paper), it would not require the teacher to facilitate a classroom discussion. Revising the goal to read, "Students will describe how to correctly apply the distributive property to solve an equation" requires students to verbalize their understanding of the distributive property and how it applies to solving the equations, which provides far more opportunities for responsive teaching during the IA. With this version of the goal, the mathematics content being taught 
remains the same, but the teacher might be less interested in students' written work and more inclined to promote small group or class discussions around the topic.

When it came to preparing for enactment, Bridget's notes on her IA protocol were similar to Julian's in that she included reminders to "listen to discussion, decide who makes most sense—call on them to explain method during discussion." However, her notes were slightly more detailed in terms of highlighting specific mathematical ideas, such as "orient them to try multiple values for $x$ " and "this means there are mult. values for $x$ " (see Figure 26).

\begin{tabular}{|c|c|c|}
\hline $\begin{array}{l}\text { Debrief: Elicit } \\
\text { and Connect } \\
\text { Reasoning } \\
\text { ( } 2-4 \text { minutes) }\end{array}$ & $\begin{array}{l}\text { You should purposefully elicit and discuss } \\
\text { strategies-to highlight common errors, } \\
\text { highlight key mathematical ideas, and/or } \\
\text { make connections. Be focused on your goal } \\
\text { and also efficient with time. } \\
\text { Call on a student to provide his/her strategy } \\
\text { or reasoning for a given solution. Whatever } \\
\text { you do, this should not be simply be "Who } \\
\text { would like to share how they did it?" } \\
\text { As the student shares, record their reasoning } \\
\text { (you should have thought about recording in } \\
\text { your planning). Elicit additional ideas from the } \\
\text { student as needed. Orient other students to } \\
\text { ideas by asking students to restate, } \\
\text { agree/disagree, ask questions, etc. You } \\
\text { should do this mostly around ideas that are of } \\
\text { core importance to your goals. Revise } \\
\text { thinking/recording as needed. } \\
\text { Elicit additional strategies in the same } \\
\text { manner as needed. }\end{array}$ & $\begin{array}{l}\text { - Follow up on } \\
\text { variety of } \\
\text { methods. } \\
\text { Compare } \\
\text { Solutions } \\
\text { - orient them } \\
\text { to try multiple } \\
\text { lalues for } \\
\text { x half room } \\
\text { may try } \\
\text { neg. \#, } \\
\text { ralf pos. } \\
\text { \# } \\
\text { - This means there } \\
\text { are mult. values } \\
\text { for } x\end{array}$ \\
\hline
\end{tabular}

Figure 26. Bridget's School GOP Protocol Notes.

These mathematical notes on her protocol provide evidence that she had given consideration to the mathematics she wanted to highlight during the IA, as well as ways she planned to go about it. 
Enactment. Bridget began her enactment by writing the problem on an interactive whiteboard. She asked the students to decide which type of equation this was and to solve the equation. Although she did not specify this in her instructions, students worked independently as Bridget circulated throughout the room, looking at students' papers. After a few moments, she asked the students to discuss their work with a partner, emphasizing that students should discuss the rationale for their answers: “...talk about the answer you got, why you think it's that answer. Compare and see if you have the same thing." Again, Bridget circulated throughout the room, moving slowly but constantly, occasionally pausing briefly to look at student papers. [This is one place in the enactment when the video skips.]

Bridget then began to call on particular students for their answers to the problem. She seemed to have strategically chosen the students on whom she called because after eliciting three different answers, she said, "I think that was all the answers I saw, but did anyone else get anything different?" This question elicited one more answer from a fourth student. Two of the answers were correct, two were incorrect.

Bridget first asked Quincy, who had given the incorrect answer of $x=1$, to explain his solution process. She elicited the process from him step-by-step, making no assumptions about his thinking, even to simplify using basic arithmetic. For example, when Quincy said he "did the distributive on the left," Bridget responded by asking, "Okay, so what did you get when you did the distributive property?" At Quincy's final step, $8 x=8 x$, he explained that he divided both sides by 8 and got $x=1$. Bridget asked, "Do we agree with this statement? $8 x$ divided by 8 equals $8 x$ divided by 8 is equal to $x=1$ ? Agree [gestures with a thumbs up, indicating how students should respond if they agree] or disagree [gestures with a thumbs down]?" The video does not allow us to see the students' thumbs, making it unclear whether there was a majority of students 
who chose one way or the other. Bridget looked around the room for a moment, then said, "All right. Let's go do some more."

For a second solution process, Bridget called on Joseph, who had given a correct answer of $27=27$. Similar to the elicitation of the first solution, Bridget wrote on a new screen only what Joseph said, eliciting details as necessary. At the conclusion of this solution, Bridget said, "So we don't have anything for $x$ for that one, but that's okay." Without further discussion, she immediately asked Kristy to describe her solution process.

Kristy had given a correct answer of $x=x$. On a new screen, Bridget carefully recorded Kristy's steps and elicited details as necessary. She asked the class, "Do we agree with that statement? That $8 x$ divided by 8 and $8 x$ divided by 8 cancels out and leaves you with $x=x$ ?" No audible or visual response from the students is apparent in the video.

Bridget then switched back to the screen with Quincy's solution, which resulted in $x=1$, and asked, "Okay, so do we agree with this statement now? Yes [thumbs up] or no [thumbs down]?" Student responses were not visible. Bridget then called on Kaden to "defend your answer." With the incorrect solution still displayed on the board, Kaden explained that Quincy “didn't keep the $x$ on the right side. If he had kept the $x$, it would have been $x=x$." Bridget responded by revoicing Kaden's idea: “Okay, so you're saying that he just kind of pretended like that $x$ wasn't there and got $x=1$ ? Is that kind of what you were thinking, Quincy?" Quincy did not respond audibly and he cannot be seen in the video, but it seems that he responded affirmatively. Bridget then displayed the third solution, which resulted in the answer $27=27$, and asked, "What do we think about this one? It's a little different. Do we agree with it [Bridget puts her thumb up]?" Students did not respond audibly. The exchange below immediately followed to conclude the IA: 
Bridget: We disagree with that one $[x=1]$. But we still agree with this one $[27=27]$. So what does that mean?

Student: There's infinitely many answers?

Bridget: Yeah, there's infinitely many answers. So my question for you...where is an okay place to stop? Which step tells you that there's infinitely many solutions? [Bridget numbers the steps of the solution that resulted in $x=x$ using the digits 1 - 4.]... Hold up your fingers for which step you think it's okay to stop. [Some of the students who are visible in the video held up two fingers.]...Okay, good. So when we see that one side is equal to the other side that means you can stop and there's infinitely many solutions. Make sense? [No student response] All right."

Commentary. Bridget demonstrated development as a responsive teacher during her school enactment. She attended to the details of students' ideas by carefully recording what they said and asking for more details as needed, rather than filling in missing details herself. Her choices of which students to call on and the order in which to discuss solutions helped her to create opportunities to enact responsive teaching moves, such as Ask $S$ if they Agree/Disagree.

Bridget's frequent use of Ask $S$ if they Agree/Disagree stands out in her School GOP enactment. Bridget enacted this move only twice during her four IA rehearsals; both instances were unresponsive because Bridget asked students if they agreed with correct reasoning without ever asking why they agreed. In contrast, Bridget enacted the move four times during her school enactment, with two of those instances being responsive. That data alone shows Bridget's development as a responsive teacher. 
Recall that, during their rehearsals, TCs tended to ask students if they agreed (without the option to disagree) with a correct answer. In her two unresponsive instances of Ask $S$ if they Agree/Disagree during School GOP, Bridget enacted the move this way. In the two responsive instances of the move, however, is where we see her development as a responsive teacher. In the first instance, she gave students the option to agree or disagree with Quincy's incorrect solution. This slight shift (to offer both agree and disagree as possible responses) was considered responsive, but only because Bridget seemed to make a deliberate choice not to further discuss Quincy's solution at that moment. Instead, she revisited his solution toward the end of the IA. In this final instance of Ask $S$ if they Agree/Disagree, she again asked students if they agreed or disagreed, then called on Kaden to "defend your answer." This instance was responsive because she heard from Kaden why he disagreed with Quincy's solution. As discussed in response to RQ1, Ask $S$ if they Agree/Disagree is a move that really cannot be responsive on its own; understanding why students agree or disagree is key.

Bridget demonstrated development as a responsive teacher during her school enactment. Like Julian, though her goals could have been written in ways that would have been more supportive of responsive teaching, she did record student ideas responsively. She also responsively revoiced student ideas, similar to her rehearsals in methods. In her planning, she anticipated a variety of solution strategies and wrote detailed notes in her planning protocol with reminders as to how to highlight the mathematics. While Bridget responsively enacted most of the teaching moves she attempted, she did not attempt as many moves as Grace or Charity, which will be described in the remaining vignettes.

Grace. Grace's School GOP enactment took place in an eighth grade classroom. Her IA lasted approximately 20 minutes, from start to finish. Only six students are visible in the video 
recording, but she called on several students who cannot be seen, suggesting that this class had 15-20 students participating in the IA.

Planning materials. Of the TCs in this study, Grace requested the most extensive help in planning her School GOP IA. She shared both her problem and goals with me ahead of time and asked me to help her make adjustments as necessary. We kept her problem the same, but instead of asking students to evaluate at $x=2$, I suggested asking them to evaluate at $x=-2$, because that might surface a range of underlying conceptions about evaluating expressions involving exponents with a negative value for $x$ (see Figure 27). I also suggested that she add a third goal involving students' abilities to correctly evaluate the expression.

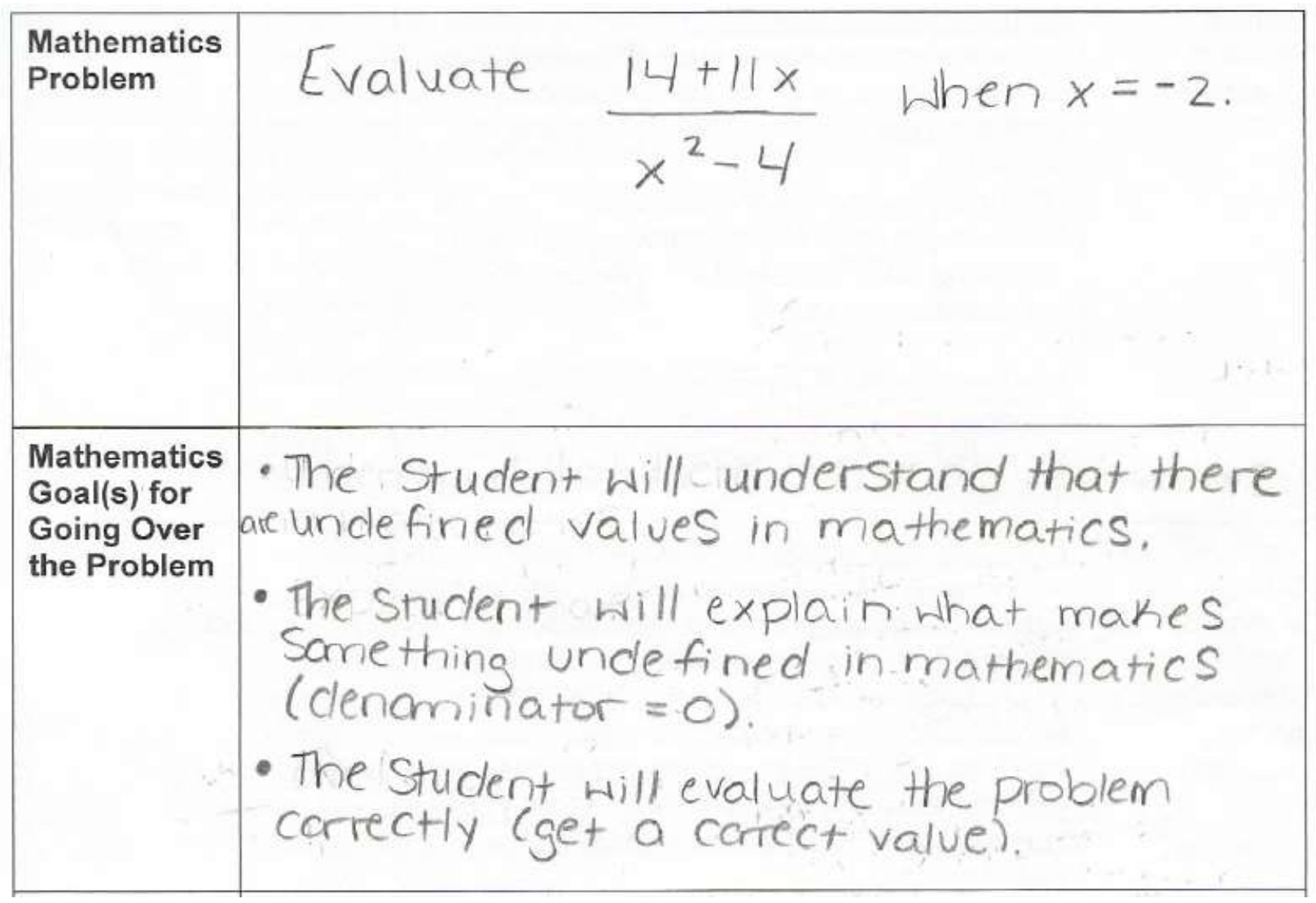

Figure 27. Grace's Problem and Goals for School GOP. 
Although I suggested that Grace add a third goal, we did not discuss the wording of her goals. Grace's first goal called for students to "understand" rather than to perform an action that would demonstrate their understanding. It is impossible to know whether students understand something without some sort of action on their part (e.g., explaining a solution in writing or orally). Her other two goals explicitly stated what she expected students would be able to do by the conclusion of her IA.

Thoroughly anticipating student responses can help TCs avoid being surprised by unexpected strategies. Grace submitted a very thorough set of anticipated student responses, which included multiple correct and incorrect strategies, sometimes with written notes about which step(s) of the problem seemed likely to cause difficulty for students (see Figure 28). Grace's experience in working with these students throughout the semester may have helped her to better anticipate student reasoning; because she had been working with this group of students on this topic, she likely had some ideas of what they might find challenging about this problem. 


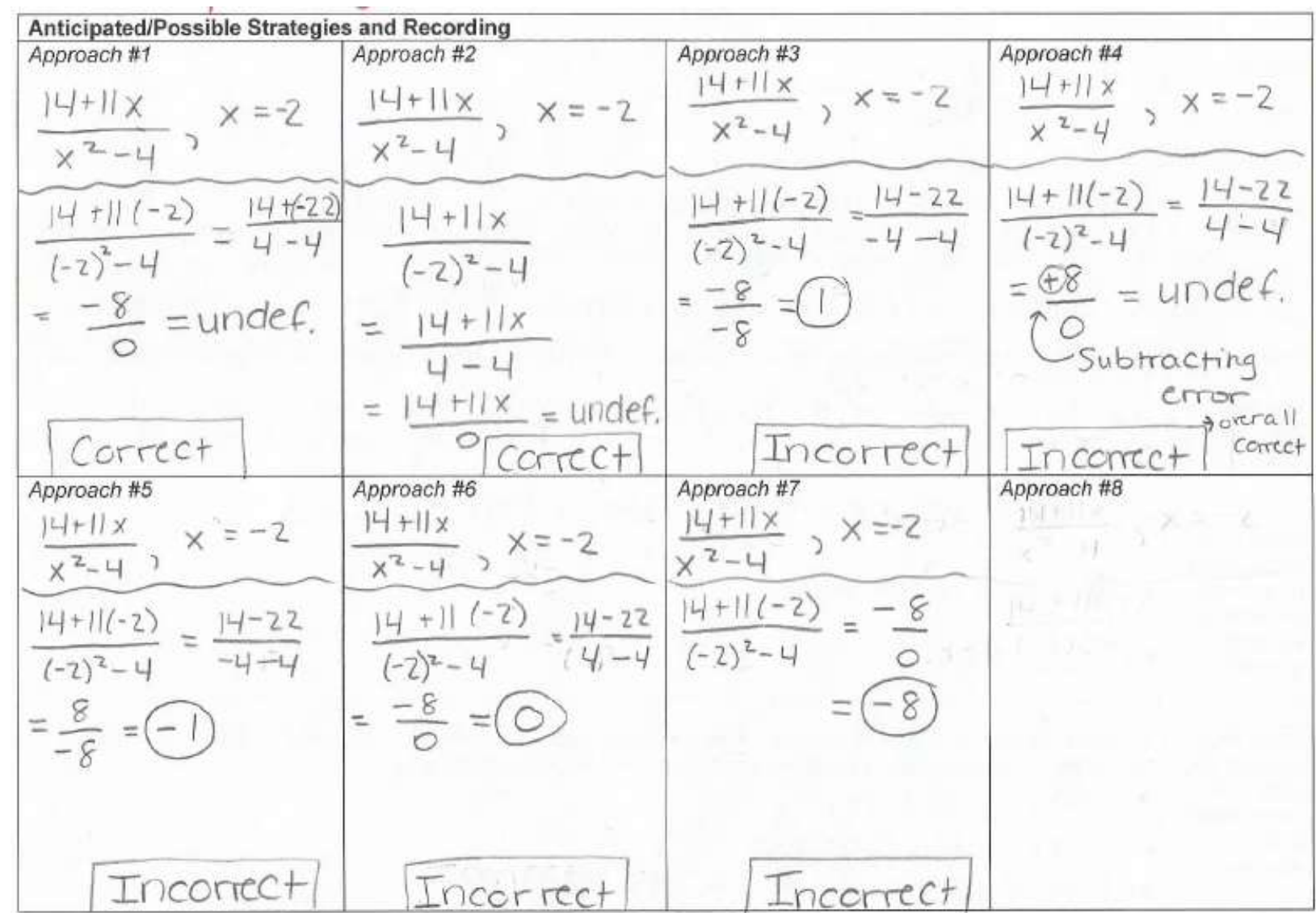

Figure 28. Grace's Anticipated Student Responses for School GOP.

Recall that Grace's notes on the planning protocols for her rehearsals were minimal or absent for the final two IAs during methods. This remained true for the School GOP IA — Grace did not write a single note on her planning protocol. As a result, it is difficult to know exactly what moves she hoped to enact or specifically how she planned to accomplish her mathematical goals during the IA.

Enactment. At the start of the IA, Grace already had the problem written on the board. She began by reading the problem aloud and then asking the students to work individually to solve the problem. As the students worked, Grace circulated throughout the room, pausing occasionally to look at students' papers. After approximately two minutes, she asked the students to discuss both their answer and their process with a partner. Again, Grace moved throughout the 
room, pausing to look at students' papers as the students worked in groups. As Grace gathered the students' attention, she elicited three different answers to the problem: $0,8 / 0$, and -8 .

Grace first called on Andy, who had given the answer 0, to share his process first. As he described his steps, Grace recorded only what he said, pausing or asking him to repeat as necessary to ensure she had accurately represented his process. With no further discussion about Andy's idea, Grace called on Brian, who had given the answer 8/0, to share his process. Again, she recorded carefully, pausing or asking him to repeat his steps to ensure accurate recording.

Grace then asked the students to individually write down their thoughts about the two different solutions displayed on the board. Grace circulated through the room, looking at students' papers. After approximately one minute, she asked students to discuss what they had written with a partner. Again, Grace moved through the room, pausing periodically and looking at students' papers.

As Grace drew the students' attention to the front of the room, she asked, "So, do we agree with this?", though it was not clear what she was referring to with "this." Several students responded simultaneously to say whether they agreed or disagreed with one solution or the other. She then asked, "Why do we disagree with [the one that resulted in 8/0]?" One student pointed out an arithmetic error, which made the answer $-8 / 0$ once it was corrected.

She then asked about the solution that resulted in an answer of 0 :

Grace: What about this one? Do we agree or disagree? [Several students shouted responses simultaneously.]

Student 1: I agree.

Student 2: I disagree.

Student 3: I agree with Andy. 
Grace: Okay, why do you disagree?

Student: Because-like, why? [other students laugh]

Grace: Okay. So in both of these problems, we get the $-8 / 0$, but here we just stopped. So we don't really know what to do after we get the $-8 / 0$.

Grace then asked the class to individually consider 0/-8. After about 20 seconds, she asked students to share their thoughts with a partner. Once students were working in groups, Grace circulated through the room for approximately 30 seconds before calling students' attention back to the front of the room. She called on Rory, who said that $0 /-8$ is equal to 0 :

Grace: Do we agree or disagree?

Students: Agree.

Grace: This is like saying - Shh. In order to simplify this problem, we think 'What times $-8=0$ ?' which is 0 . So now, let's think about $-8 / 0$...I want you to think about the answer to this problem now. [Students began to talk.] Think about it individually.

Student: It has no value.

Grace: Think about it individually and when you have some thoughts, go ahead and talk with your partner. [Grace circulated through the room once, then went back to the board as students finished their conversations.]

Grace: This is like saying 'what times 0 equals -8 ?'

[Several students responded simultaneously.]

Student 1: -8

Student 2: Nothing!

Student 3: Nothing times 0 equals [inaudible]. 
Student 4: It's physically impossible!

Grace: Does everyone hear what Andy is saying?

Student 5: No! I cannot!

Grace: Everyone, turn forward. Listen to Andy and Henry, they're both saying the same thing. Andy, go ahead.

Andy: Well, anything times 0 will equal 0 , so 'What times 0 equals -8 ' is already physically impossible. You cannot do it.

Grace: Okay, Henry, what were you saying?

Henry: It has no value.

Grace: Okay, so when there's not a value, there's not something defined, what do we say in math? I want you to think about it to yourselves. [Students are talking, some seemingly to themselves.] Think about it to yourselves first. Not a value in math. [Students still talking.] I want you to think about it to yourselves. [Students still talking. Grace paused for a moment.] Then when you have some ideas, share them with your partner.

Once students were talking with their partners, Grace circulated through the room, looking at students' papers.

Grace: Okay, so Alex, what did your group talk about?

Alex: $\quad$ We talked about how it wasn't real and that it was fake.

Grace: So what do you say? You were saying it back there. Do you remember?

Alex: $\quad$ No.

Grace: You said that there was no solution.

Student: It's unde-something. 
Grace: Unde-something? [laughs] It's undefined!

Grace then wrote 'undefined' on the board. (Her completed board work may be seen in Figure 29.)

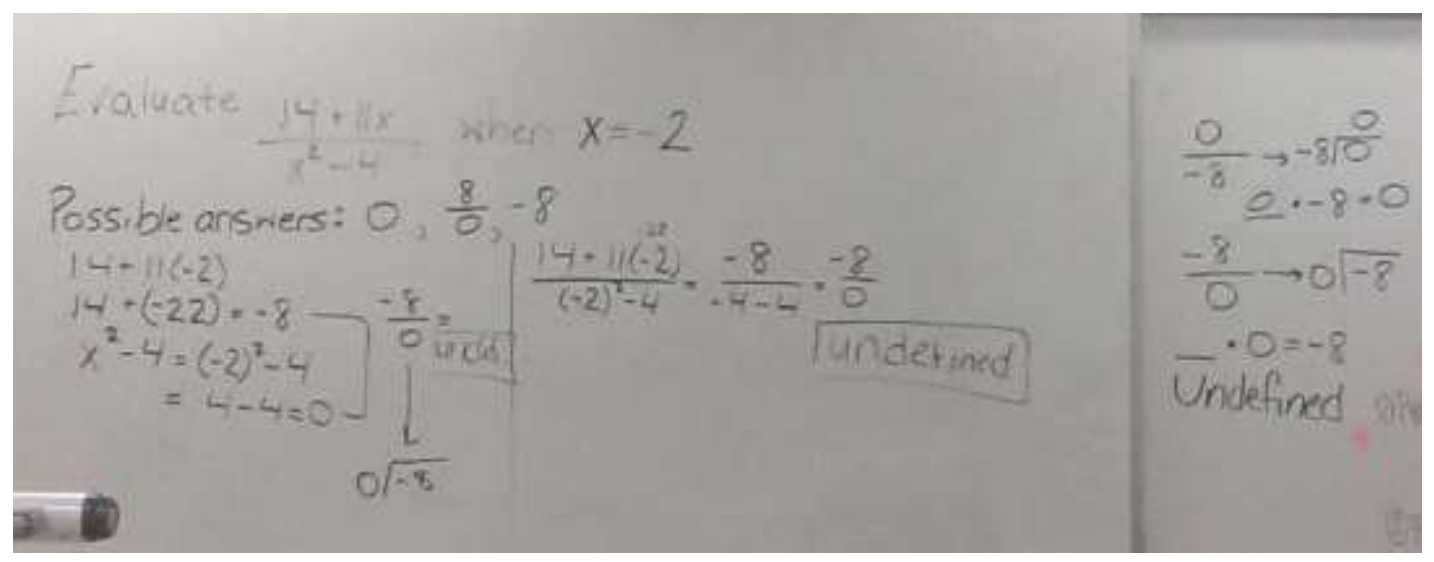

Figure 29. Grace's recording of student solutions during School GOP

Then, Grace asked a different student to revoice the solution to the problem:

Grace: Okay, so real quick, I want Heather, will you explain to me how to correctly do this problem? Just real briefly.

Heather: What do you mean 'correctly solve this problem?'

Grace: Like what did we do in this situation to solve the problem?

Heather: I don't know.

Grace: All right. Do you want to ask somebody? Phone a friend?

Before Grace managed to get Heather to revoice the process used to solve the problem, Andy brought a question forward by saying, "It didn't say it could be undefined." Grace was unsure what he meant by that, and in the moment, Andy said he could not explain it any better. This issue was tabled momentarily. In the meantime, Grace asked the class to look back at the first two processes to see if they agree or disagree. She gave instructions for students to think individually first, then when they had an idea, they were to share with a partner. As the students 
were working, Andy called Grace back over and they had a brief conversation about a difference between evaluating an expression and solving an equation. Grace returned to the front of the room:

Grace: Do we agree?

Student: With what?

Grace: With both of them? They're the same thing. Do we agree that they're both undefined?

Students: Yes.

Grace then called on a student to explain the process that was used to solve the problem. He described how to plug in the value for $x$, simplify, and then identify the answer as undefined. She concluded the IA by asking Andy, "Can there be undefined values in math?" to which he replied, "Yes." Then she asked Henry, "When can we get undefined values in math?" Henry replied, "When you divide by $0 . "$

Commentary. For Grace's School GOP enactment, the students' enthusiasm to share ideas (sometimes to the point of being slightly disruptive), was one notable difference between her rehearsals in methods and her School GOP. The students in Grace's class were especially eager to work together and share their responses during her School GOP IA. During her postinterview, Grace commented on this as one aspect of her IA that did not go as planned; in her experience, it was usually difficult to get these students to participate during class. She went on to say that this was the reason she permitted their somewhat chaotic shouting of answers and the group conversations during individual think time — she wanted to avoid stifling this rare showing of enthusiasm. This type of tension may arise anytime a teacher engages students in class discussion or group work. The fact that Grace did not have to manage this type of student 
behavior during her rehearsals in methods, however, may have contributed to the difficulty she had in managing the behavior of the eighth graders.

Grace enacted some teaching moves in ways that were similar to Julian and Bridget. Although she usually questioned students in ways that allowed them to share their thinking, her attempt to get students to say the word "undefined" led her to ask questions that pushed students toward her thinking, which is similar to some of Julian's questioning strategies. Like Bridget, she enacted Ask $S$ if they Agree/Disagree with some success. I will further discuss Grace's use of this move later in the commentary.

Perhaps the most unique aspect of Grace's School GOP IA was her frequent use of $T$ Prompts $S$ to Take Think Time, particularly when the IA did not call for it. She enacted this move more than any other TC during School GOP. Recall that, during methods, my coaching interjections around this move suggested that the teacher provide think time (1) as students were reflecting on the lesson or (2) when the students did not seem to know how to respond to a prompt. Grace did not enact the move in either of these ways during her school enactment. Instead, she prompted students to take individual and/or group think time to (1) compare two solution methods on the board; (2) consider the problem $0 /-8$; (3) consider the problem -8/0; and (4) consider what we say in math when something has no value.

Grace's enactments of T Prompts S to Take Think Time provide one example of how my attention to different contexts (i.e., methods course and schools) allows for the consideration more nuanced ways to view certain moves. Her use of T Prompts $S$ to Take Think Time progressed from more responsive, to less responsive, to unresponsive. The first enactment of this move gave students substantial freedom to come up with their own ideas. There were multiple perspectives that would have allowed students to reach the correct answer. In the second and 
third enactments, the prompts Grace gave the students were more like mathematical facts to be explained, rather than open-ended questions that allowed for creative student thinking. The last enactment of this move saw Grace essentially asking students to come up with the term undefined on their own, which was unresponsive for its focus on Grace's idea rather than the students' ideas. These progressive shifts in Grace's enactments of T Prompts S to Take Think Time may have partially been in response to her goals of the IA. Since one of her goals was to have students explain what makes something undefined in math, she may have felt the need to introduce the term to students. However, a more responsive way to do this might have been for her to state the term for students, but then allow them some time to think about how to explain why $-8 / 0$ is undefined.

Another teaching move of interest in Grace's School GOP IA is Ask $S$ if they

Agree/Disagree. She enacted the move in a slightly different way than Bridget; instead of asking whether students agreed with a correct answer, Grace sometimes asked if students disagreed with an incorrect answer. It is interesting that in both of these instances, several students vocalized responses of both agreement and disagreement with the idea in question, but in both instances the idea was incorrect and Grace chose to follow up with the students who said they disagreed with those ideas. These efforts to nudge the IA along were not necessarily unresponsive; as has been discussed in previous sections, that determination is dependent upon whether Grace asked students to explain their choice. Note that, in the second instance, Grace asked why the student disagreed, but he responded, "Because—like, why?" Rather than pushing him to articulate exactly what was wrong with the process, she explained it instead. Thus, despite her effort, this exchange was considered unresponsive. 
Finally, Grace attempted to enact Ask Different $S$ to Revoice, but with no success. This may partially be a result of the lack of planning notes on her IA protocol. It is not clear why Grace chose not to write any notes, but the following excerpt from her post-interview suggests that her enactments of Ask Different $S$ to Revoice might have better met her expectations if she had written a few reminders for herself: “... I almost wish you would have been there when I was doing it in the schools because I forgot to have them restate. I was watching my video, I think I might have once." Although she did not completely forget to have students revoice, it seems that she intended to enact that move more often and/or more judiciously. Grace's reminders in her protocols to "have someone restate/rephrase" (Figure 14) early in the semester proved useful during her rehearsals. It is reasonable to conclude that similar reminders on her School GOP protocol would have helped her better achieve this goal she had set for herself.

Another difficulty with Grace's enactments of this move involved the student responses she received. For example, it was clear that Heather was not interested in revoicing the correct process for solving the problem; she first delayed answering the question before eventually just stating, "I don't know," which prompted Grace to move on to ask another student. Grace identified this moment as challenging in her video annotation. She wrote, "This student does not like to answer or explain things for the class. She normally answers short and refuses to explain the material. I thought that she might act differently in this activity because many of the students were." Heather's response was unlike any Grace had received during methods; the other TCs always made an effort to revoice when asked. Although, in some sense, she expected Heather to respond this way, the extreme difference in the student response undoubtedly caused Grace some difficulty in deciding how to respond. 
Grace's school enactment displayed some development as a responsive teacher, while also highlighting areas for continued improvement. She made repeated use of $T$ Prompts $S$ to Take Think Time, but almost to the point of overuse - allowing group think time for students to come up with the word undefined was not the most judicious use of the move. Like Bridget, she had a tendency to enact Ask $S$ if they Agree/Disagree in ways that subtly led students to desired conclusions (i.e., specifically asking if they disagreed with an incorrect solution). She demonstrated strong planning abilities, particularly establishing goals and anticipating student strategies, but making no notes on her protocol may have contributed to the IA not fully proceeding as she hoped. More written notes on the protocol might have helped her to more carefully think through how and when to best enact responsive teaching moves, such as Ask Different S to Revoice. In the final vignette, we will see that Charity enacted this move, as well as several others, in responsive ways.

Charity. Charity's School GOP enactment took place in a sixth grade classroom. Her IA lasted approximately 24 minutes, from start to finish. Approximately 20 students participated, some of whom are enrolled in special education. As a result, this class also usually has a resource teacher in the room, in addition to the classroom teacher. During Charity's enactment, however, the resource teacher was not present.

Planning materials. The problem Charity chose to use for her School GOP IA asked students to interpret a bar graph to find the fraction of students who chose video games as their favorite hobby (see Figure 30). She asked me ahead of time if this type of problem would be appropriate for the School GOP IA; I agreed that it would work with no alterations. Beyond this, we did not discuss other aspects of her planning (e.g., goals, anticipated student reasoning). 

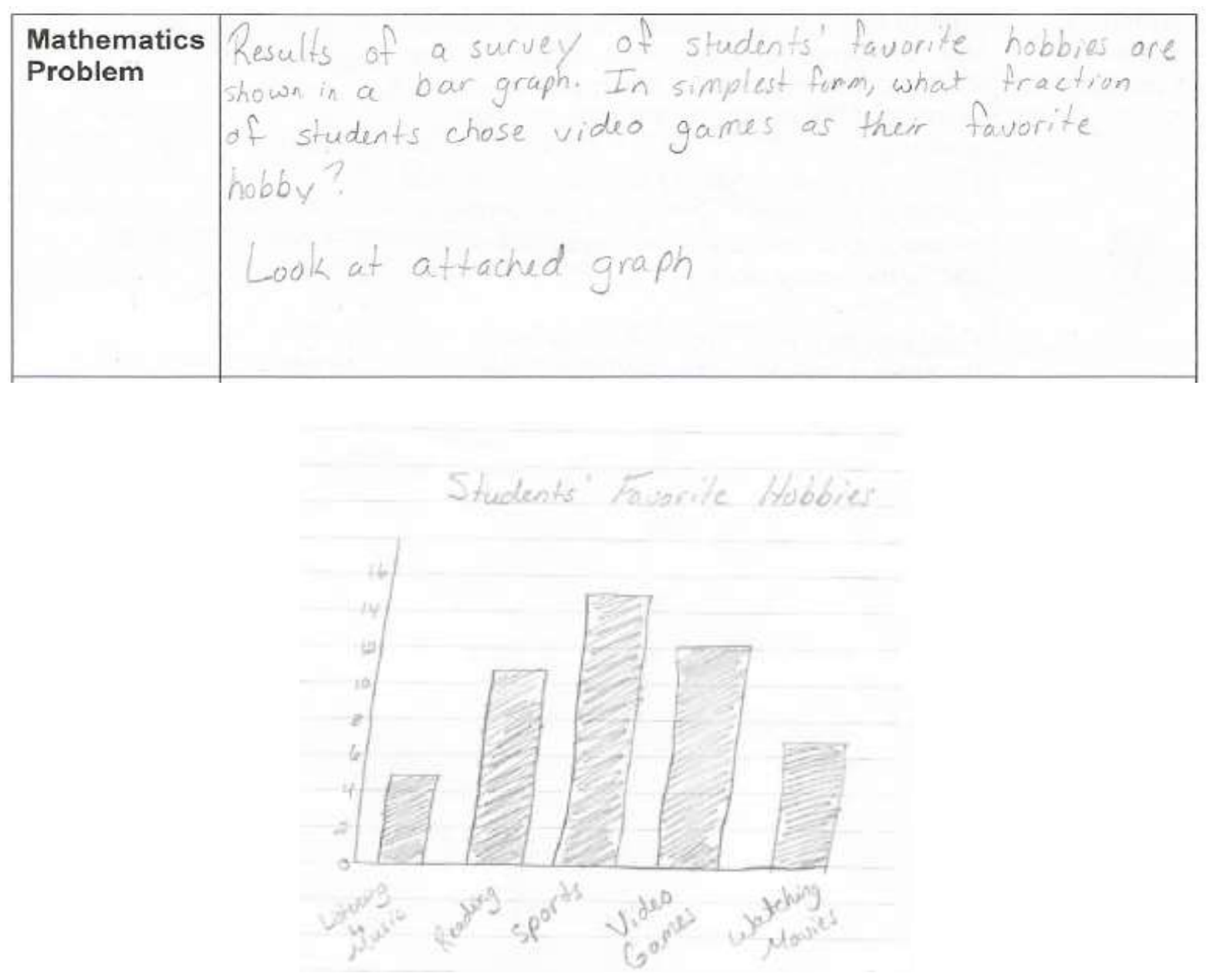

Figure 30. Charity's Problem for School GOP

Similar to the goals written by other TCs, Charity's goals could have been written to explicitly describe what the students should be able to do. Charity had three mathematical goals for her IA (see Figure 31). 


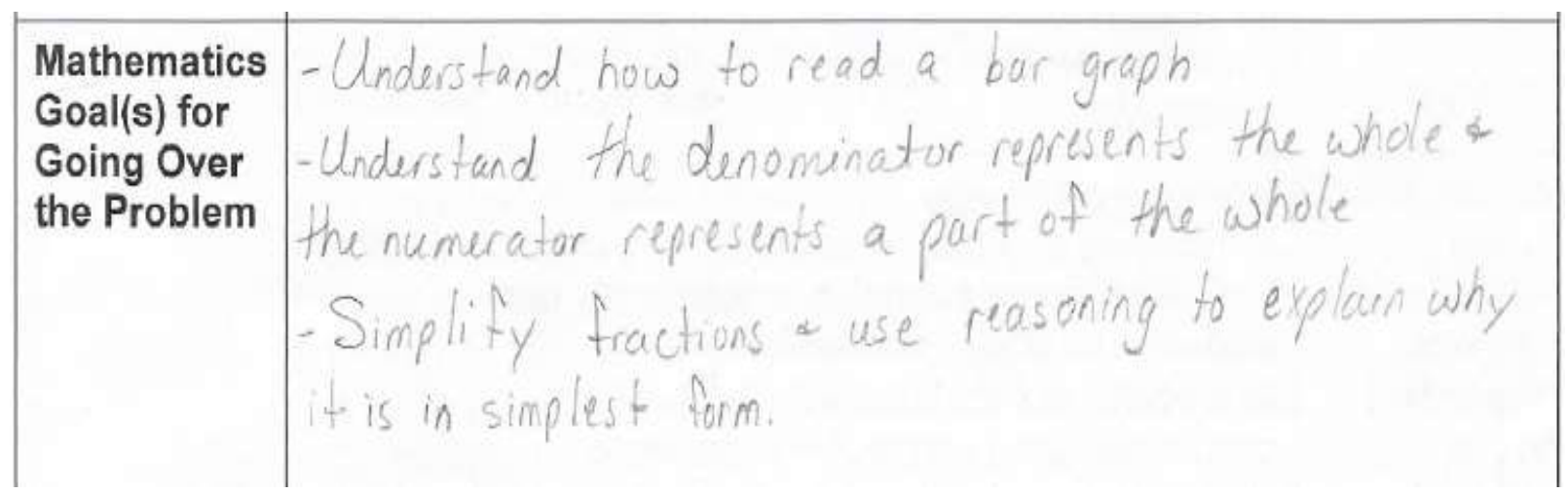

Figure 31. Charity's Goals for School GOP

Similar to Grace, two goals stated that students would "understand" something, which is problematic because these goals do not require any evidence of students' understanding. Her third goal, however, did a better job of conveying exactly what skills the students should demonstrate (i.e., simplify fractions) and how the students would demonstrate that knowledge (i.e., "use reasoning to explain why [the fraction] is in simplest form").

Also, like Grace, Charity submitted a thorough set of anticipated student responses (see Figure 32). For the School GOP IA, she included two correct approaches that involved different processes for simplifying the fraction. She also included 5 incorrect approaches, along with a few words or phrases to describe what error in student thinking would be made apparent with each incorrect approach (e.g., "If student just looks at the y-axis, they may think that the total number of students is 16 "). This thorough set of anticipated student responses likely helped Charity gather ideas of strategies and mistakes to watch for as students were working, as well as to make decisions about which strategies or mistakes to highlight during the class discussion. 


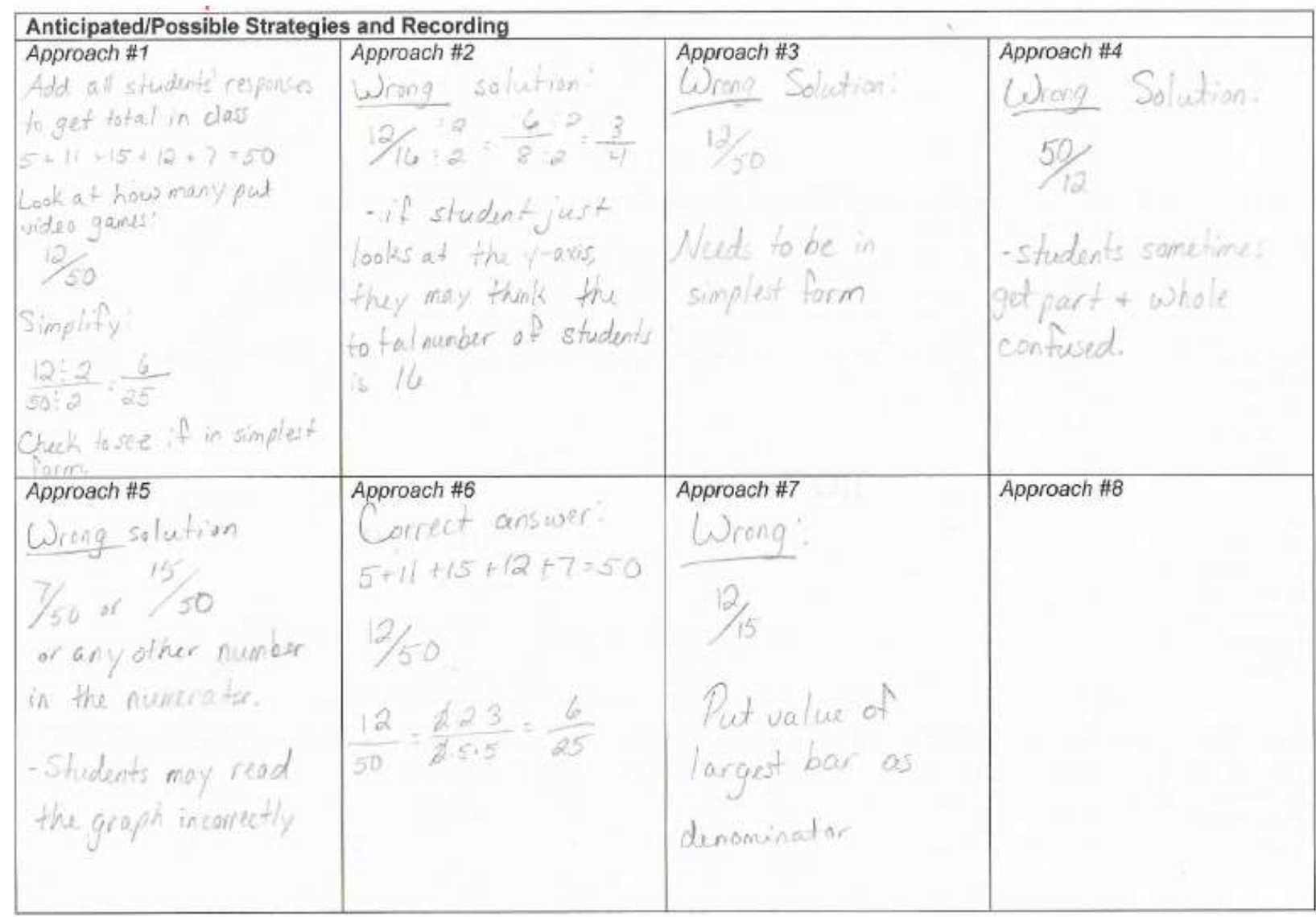

Figure 32. Charity's Anticipated Student Responses for School GOP

Charity's planning protocol notes were more extensive and more detailed in terms of specific moves to be made at particular moments than the notes written by other TCs for their school enactments. Consistent with her planning work for the IAs in methods, she included reminders to "Think-Pair-Share" and "Have others restate - try not to just pick people around them." Her emphasis on these specific responsive teaching moves is apparent in her enactment, which will be discussed in the next section.

Enactment. Charity began her enactment by asking students to open their textbooks to view the problem. After the class read the problem in unison, she asked students to identify important parts of the word problem. The students shared responses such as "video games" and "fraction in simplest form," which Charity wrote on the board. Then, she asked the students to 
solve the problem and to give her a thumbs up when they were finished. As students worked individually, Charity walked around the room, looking down at students' papers. After a few moments, three students raised their hands. Charity walked toward them, but said loudly enough for the entire class to hear: "I'm not going to let you know if it's right or not. I'm just walking around to see what we're doing." The three students slowly lowered their hands and continued working.

After a few more moments, Charity asked the students to talk the problem over with a partner. She asked them to discuss the answers they got, as well as how they arrived at those answers. During this time, Charity quickly walked around the room, briefly looking at some students' work. She regained the students' attention by doing a rhythmic clap that they echoed. Then, Charity invited students to raise their hands to tell her what answers they got. Three students responded, each with an incorrect answer. Charity recorded their responses of $\frac{3}{25}, \frac{1}{3}$, and $\frac{3}{4}$ on the board.

Charity first called on Daniel, who got $\frac{3}{4}$, and asked him to explain how he got his answer. With some prompting from Charity to explain the details of his solution, Daniel explained that since 12 students liked video games and there were 16 students in all, he began with the fraction $\frac{12}{16}$. To simplify, he divided by 4 . Charity revoiced Daniel's reasoning, then asked, "What do we think of that? Do we agree? Disagree? Raise your hand if we have an answer. [short pause] Okay, what do you think, Daphne?” Daphne responded, “I disagree. I don't know why. I just have a different answer."

At this point, Charity shifted the conversation from a consideration of the answer $\frac{3}{4}$ to a consideration of Daphne's different answer, $\frac{3}{25}$. Daphne explained that she started with $\frac{12}{100}$, then 
simplified by dividing by two and then dividing by two again. When Charity asked why she used 100 in the denominator, Daphne could not provide a mathematically sound explanation and eventually said, "I don’t know." Charity asked the class, "What do we think of that?" She called on Nicole, who had raised her hand. Nicole responded that she added the number of people who preferred each hobby to get a total of 50 people.

At this moment, Charity paused Nicole to ask all of the students to add up the numbers Nicole mentioned. She asked the students to give her a thumbs up when they had an answer. While students worked, some individually and some in small groups, Charity remained at the front of the room. After a few moments, she called their attention to the front and began to work through the addition at the board. After correcting a recording error and giving the students a few more moments to add the numbers, she asked the students to "Show me on your hands what you got." Several students held up five fingers on one hand and made a zero with their other hand. Although Charity saw "a lot of 50s," she went on to add the numbers at the board, eliciting periodic responses from the class. Once the entire class seemed to be in agreement that the sum of the numbers was 50, Charity returned the class's attention to Nicole by asking her to explain what the 50 means. Nicole explained that the 50 represented "the number of students in the whole class." Charity then asked Brandy to restate:

Charity: Brandy, can you restate why we did this? Why did we add all these up?

Brandy: So we'd know how many kids are in the classroom.

Charity: So we'd know how many kids in our classroom. How many in our classroom?

Brandy: 50.

Charity: $\quad 50$. Okay, keep going, Nicole. 
Nicole explained that she put 12 in the numerator, 50 in the denominator, and then simplified. Charity asked her to describe how she simplified. Nicole responded that she divided both the numerator and the denominator by 2 to get $\frac{6}{25}$, an answer that was not stated at the beginning of the class discussion. Once again, Charity asked someone to revoice:

Charity: Can somebody restate what Nicole just said? How we solved this problem? David, Can you restate what Nicole did?

David: $\quad$ I got $\frac{3}{4}$.

Charity: Okay. So you solved it like Daniel did. [Kristin raises her hand.] Go ahead, Kristin.

Kristin: She added up all the people and [inaudible] and then she got 50 then she put [inaudible] in the denominator and then she divided it by 2 .

After Kristin's revoice of Nicole's idea, Charity turned the conversation back to how students arrived at the answer of $\frac{3}{4}$ :

Charity: Okay. Let's revisit what Daniel and a few others have done. Why did we choose to use 50 instead of 16 ? Go ahead, Fiona.

Fiona: Because each of the kids, if you look at the number of hobbies and the number of students for them, it doesn't add up to 16 .

After Fiona's explanation, Charity asked once more for someone new to revoice the process used to solve the problem. Several students asked what the right answer was. Charity responded, "I don't know, what do we think the right answer is?" A few students answered, “ $\frac{6}{25}$." Charity then called for the students to vote for which answer was correct, $\frac{3}{4}$ or $\frac{6}{25}$. Most students voted for $\frac{6}{25}$, but there were at least two who chose $\frac{3}{4}$. Charity questioned the students about why it was 
necessary to use 50 for the denominator. Daphne responded, "Because 50, if you add up ALL the kids, you get 50. And then you have to take how many people want video games out of the 50, divide that, and that gives you your answer, $\frac{3}{4}$." Charity then asked David, who believed $\frac{3}{4}$ to be the correct answer, to revoice Daphne's explanation.

Charity: Okay. David, can you restate what she just said?

David: I can restate how I got my answer.

Charity: Okay, why did you get your answer then?

David: So, I figured because there was 12 kids that liked video games, and the graph only went to 16 . I did $\frac{12}{16}$, divided that by 4 , and then I got $\frac{3}{4}$.

Charity: Okay, someone explain to David why we're not using 16 again. Go ahead, Baylee.

Baylee: Because that's how many people like one of these hobbies and it's not that, it's the 12 people, it's not the 16 .

Charity: Nicole, do you have something to add?

Nicole: If you re-look at the problem, it says, in simplest form, what fraction of the students chose video games. Out of all the students, if you added them, you get 50, and that's how much students there are in all. And, if you can look at the video games in there, there's 12 students out of 50, the fractions of the students chose, that's how you know.

Charity revoiced Nicole's statement, then asked another student, Frankie, to explain how he got $\frac{12}{50}$. Frankie accurately explained the idea and Charity then shifted the discussion to focus on 
whether $\frac{6}{25}$ is in simplest form. One student said that it was simplified because 6 and 25 have no common factors.

Following this discussion about simplifying, Charity again asked for a different student, Sam, to restate the process used to solve the problem. Sam said that they added, then Charity prompted him with questions, such as "What did we add?" to help him articulate the details of the process.

In the final four minutes of the IA, Charity asked students to solve a similar problem as a way to check their understanding. Using the same graph, she asked students to identify, in simplest form, the fraction of people who chose sports as their favorite hobby. Students were instructed to give a thumbs up when they had solved the problem. As students worked individually, Charity circulated throughout the room, looking at students' papers, generally ignoring students' raised hands, and periodically reminding students to show "how you got there."

As she drew students' attention back to a whole-class discussion, she called on Lexi to give her answer to the problem. Lexi gave $\frac{3}{10}$ as her answer. After Charity asked her to explain her process, Lexi explained that since 15 liked sports and there were 50 total people, she first wrote the fraction $\frac{15}{50}$. Then, she divided by 5 .

Charity: What made you choose 5 ?

Lexi: $\quad$ Because the division rule says that if you want to do it by 5 , then it has to be 5 or 10 as the ending number.

Charity: So 15 divided by 5 is?

Lexi: $\quad 3$. 
Charity: And 50 divided by 5 is?

Lexi: $\quad 10$.

Charity: Do we agree or disagree?

Students: [in unison] Agree.

Charity: Okay, if we agree, how'd we get there? I need another person to restate. [Several students raise their hands.] Ian, can you explain how we got here? [Ian shakes his head.] Isabella, can you explain how we got here?

Isabella restated the correct procedure for solving the problem. Charity concluded the IA by asking, "Any questions? Do we need to do another one?" There was no audible student response to either question.

Commentary. Charity involved a large number of students in the discussion around her problem, often through the enactment of the move Ask Different S to Revoice. She commented in her video annotation that she "felt the need to restate because typically that is what the resource teacher does with the special needs students in the class." It is not clear exactly how the resource teacher enacts the restate move, but based on Charity's enactments of T Repeats and Ask Different $S$ to Revoice, it seems that repetition is one of the norms for this class.

During her Teaching Demo and two IA rehearsals in methods, Charity enacted T Repeats a total of five times; during her single School GOP enactment, she also enacted T Repeats five times - only slightly less frequently than Julian. None of these instances were considered responsive. Perhaps the resource teacher uses a restate move as a means of repetition, to ensure that students have properly heard and understand instructions, which has led Charity to repeat students' words often. 
Because Charity used the word "restate" to describe the resource teacher's actions, and because Charity enacted Ask Different S to Revoice during her School GOP more than any other TC, this move also warrants consideration. Although this move has the potential to orient students to one another's ideas, based on the comment above from her video annotation, it seems that this was not necessarily her motivation for enacting Ask Different S to Revoice. Both Charity and the students likely had the resource teacher's technique in mind, which may have had an impact on how Charity and the students took up this revoicing move.

Charity enacted Ask Different $S$ to Revoice responsively several times throughout the IA, but she also found herself in some difficult situations when attempting to use this move. This was particularly true with David's multiple refusals to revoice. Charity commented about the first refusal in her video annotation: "I had no idea what to do when David insisted on telling me that he solved it a different way. I just wanted to tell him, 'Just tell me what Nicole did!'” Charity also commented about his second refusal, saying that she "couldn't really stop him" when he insisted on explaining how he got his answer instead. This is evidence of the tension that TCs may feel as they attempt to teach responsively—negotiating the need to focus on one student's idea to move toward the mathematical goal while also helping all students feel that their ideas are valued. This may have been at least part of the issue for David; he may have been less willing to reason about Daphne's idea because Charity had not yet fully engaged with his idea. It is also worth noting that David's attitude was different from Heather's attitude during Grace's IA—David kept insisting on explaining his answer rather than revoicing someone else's while Heather seemed uninterested in discussing the problem at all. However, the end result for each TC was the same; both instances were considered unresponsive because they did not succeed in engaging students with one another's ideas. 
Another, perhaps larger, issue with Charity's interactions center on her potential underlying motives for calling on David. Specifically choosing David to revoice may have served as a suggestion that his answer was incorrect and that he needed to be convinced by the other idea. While it is true that David was incorrect, Charity's teaching moves seemed to be an attempt to push David toward a particular way of thinking, which also would not be responsive.

There is evidence to suggest that Charity slightly misunderstood the purpose of Ask Different S to Revoice as it should be used in responsive teaching. Recall that Charity interrupted Nicole more than once as she shared her (correct) solution strategy. Charity commented in her video annotation that she "felt it was good to stop [Nicole] from time to time and have the other students restate, so that they're not just listening to her." While her desire to maintain students' attention is commendable, this was not a responsive use of Ask Different S to Revoice. By interrupting Nicole periodically, Charity took on a larger role in guiding the other students' thinking; her pauses highlighted certain aspects of Nicole's solution, thereby reducing the amount of time that the class discussion was student-directed.

Another move used by Charity was Ask S if they Agree/Disagree, though she used it differently than Bridget and Grace. Recall that Bridget and Grace had a tendency to ask if students agreed with a correct answer or disagreed with an incorrect answer, thereby leading students to a desired conclusion. In each of Charity's enactments of the move, however, she asked students if they agreed or disagreed with a particular idea. In the first instance, Charity called on a Daphne, who said, "I disagree. I don't know why. I just got a different answer." Rather than pushing Daphne to explain why she disagreed, Charity allowed the conversation to shift to Daphne's solution to the problem. In the second instance, when the idea was correct, several students said they agreed, but instead of asking why they agreed with the solution, 
Charity asked, "How'd we get there?" Restating the process is not the same as expressing an understanding about why it is the correct method, which rendered this instance unresponsive as well. While each of these instances got off to a neutral start, they were ultimately considered unresponsive because of the ways Charity followed up the move.

One other move, T Further Questions $S$, is worth mentioning as it relates to Charity's School GOP enactment because she was the only TC to enact this move responsively. Recall that instances of $T$ Further Questions $S$ were considered responsive if the questions left room for students to share their own reasoning about a problem or idea (e.g., "Where did you get 100?"), rather than requiring a straightforward correct answer (e.g., “And 3 x 4 is?”). In each of Charity's responsive enactments of $T$ Further Questions $S$, she asked a probing question that allowed a better understanding of student thinking. This is consistent with her enactments of the move during her IA rehearsals, when she also asked more responsive probing questions than the other TCs. It is encouraging that her ability to recognize appropriate moments in which to probe student thinking was not diminished in the school setting.

Charity's school enactment displayed development as a responsive teacher, although there is room for improvement. Like the other TCs, her articulation of the mathematical goals for the IA did not always identify what evidence the students would produce to demonstrate their achievement of the goals. Again, this suggests that more support from the TE in establishing the goals for the IA would be beneficial. However, Charity's other planning materials stood out, as her anticipation of student strategies and her protocol notes were very detailed. She enacted a wider variety of moves than the other TCs, some of which she enacted more responsively than the other TCs (e.g., T Further Questions $S$ and Ask Different S to Revoice). Charity's use of these moves is consistent with her use of the moves during the IA rehearsals, which suggests that it is 
possible for TCs to apply what is learned in methods in school settings. On the other hand, there were some moves that Charity enacted less responsively than the other TCs, such as Ask $S$ if they Agree/Disagree. Even in her flawed enactments of this move, however, we can see her development as a responsive teacher in the way that she began each enactment of this move by offering students the options to agree or disagree, no matter whether the idea in question was correct or incorrect.

Summary of TCs' responsive teaching in schools. Looking across all four vignettes leads to several conclusions about TCs' responsive teaching in school settings. First of all, the structure of the IA supported TCs in their responsive teaching. Even Julian, whose school enactment was not very responsive, still enacted some responsive teaching moves simply because they were called for in the IA protocol. Secondly, goals that are not articulated well may contribute to difficulties in teaching responsively. TCs who noted specific responsive teaching moves to be made were more likely to attempt those moves during their enactments, which illustrates the importance of TCs' planning for specific moves.

Finally, the different setting — the school classroom as opposed to the methods classroom—seemed to impact TCs' responsive teaching; TCs took up some moves differently in methods and in schools. For example, T Repeats was used less responsively in schools, while enactments of Ask S if they Agree/Disagree, although still flawed, were generally more responsive in the school setting. As part of this different setting, school students participated differently from TCs acting as students during the IA rehearsals. During methods, the students made every effort to revoice another student's idea when asked, sometimes beginning their response with, "I'll try," indicating that they did not feel fully confident in revoicing the idea. School students, however, did not always make the same effort. (Recall the interactions between 
Charity and David or Grace and Heather.) The differences in student responses also had an impact on TCs' responsive teaching. Responding to school students put TCs in some situations that were unlike what they experienced in methods, which left some of TCs' attempts at responsive teaching (e.g., enacting Ask Different $S$ to Revoice) unresponsive. Despite these differences between settings, TCs' attempts to enact responsive teaching moves in schools indicates that their participation in approximations of practice (i.e., IA rehearsals) in methods led them to be more mindful of responsive teaching in the school setting.

In Chapter 4, I reported my findings around three research questions: how TCs developed skill in responsive teaching during a methods course in which cycles of investigation and enactment were a central pedagogy, how coaching in a methods course affected TCs' responsive teaching, and finally, how extensively TCs taught responsively in school settings. In Chapter 5, I summarize the findings and provide implications for practice and future research. 


\section{CHAPTER FIVE}

\section{Discussion of Findings}

In Chapter 1 of this dissertation, I argued that this study was needed to provide insight into how TEs might help secondary mathematics TCs learn to teach responsively through a methods course designed to support TCs' development of responsive teaching skills. Responsive teaching — making students' content-specific ideas the focus of instruction—positively impacts student learning, but is intellectually demanding for teachers (Hammer et al., 2012; Kazemi et al., 2015; Richards \& Robertson, 2016; Robertson et al., 2016). Because of the challenges inherent in this work, TCs require support from TEs to begin to develop skill in responsive teaching. Such development includes enacting responsive teaching moves purposefully and at times that have the potential to maximize the responsive potential of those moves. TCs must plan to enact responsive teaching moves ahead of time, but also learn to execute those teaching moves in responsive ways. These skills are not developed one after another, but instead, simultaneously.

In a university methods course, the TE can provide TCs with pedagogical tools, such as responsive teaching moves, and help TCs learn to enact those moves judiciously, in part through the use of a pedagogy of practice that involves coached rehearsals as part of a cycle of investigation and enactment. Although TCs can develop responsive teaching skills in a methods course, in order for secondary students to reap the benefits of responsive teaching, TCs must be able to appropriately use the pedagogical tools from methods in the school setting. To better understand TCs' development in responsive teaching, my study was guided by three research questions:

1. How do TCs develop skill in teaching mathematics responsively within a methods course in which cycles of investigation and enactment are a central pedagogy? 
2. How does coaching in methods affect TCs' responsive teaching?

3. To what extent do TCs teach responsively in school settings?

I looked to previous course designs involving pedagogies of practice in teacher education to inform the design of my methods course, which was centered on a cycle of investigation and enactment. In a secondary mathematics methods course, TCs participated in four iterations of this cycle, each around a different IA. TCs' IA enactments took the form of coached rehearsals, with me (the TE) acting as coach. Each IA rehearsal was video recorded; TCs then reflected and commented on their videos in response to a series of prompts. TCs also enacted one IA in a school setting with secondary mathematics students. This enactment was also video recorded and TCs used the same set of reflection prompts to comment on their videos.

I looked to previous research designs involving pedagogies of practice, including my own pilot study, to inform the design of the current study. Videos of TCs' Teaching Demos, rehearsals in methods, and school enactments served as primary data sources for my research. Using Studiocode, I first analyzed videos to objectively code instances to describe who was talking (e.g., teacher, student). Then, I coded the videos to identify S-T and C-T exchanges which were focused on a single idea or substance. I examined the S-T exchanges to determine what responsive teaching moves were enacted and whether they were enacted responsively. A similar examination occurred for C-T instances, but instead, I looked at whether the substance of the C-T exchange was focused on responsive teaching. These analyses, along with other data sources (TCs' video annotations, three interviews per TC, and my reflective journal) were used to pursue each of the three research questions. 


\section{Summary of Findings}

I first summarize my findings around each RQ. Next, I discuss implications of those findings for the design and implementation of coached rehearsals in secondary mathematics teacher education. Finally, I describe the implications of my findings for future research, including the research design for future iterations of similar studies.

RQ1 findings. First, it is important to note that TCs' development of responsive teaching skills in a semester-long methods course means that they may enact some moves better (i.e., more responsively and judiciously), even if they do not necessarily enact all moves well. This development will likely look different for individual TCs; they may not develop in the same ways or to the same extent as their classmates. Thus, rather than suggesting that TCs completed my methods course as fully responsive teachers, my findings say more about what development might look like and the barriers that TEs and TCs may face as that development occurs.

TCs' development of responsive teaching skills took a variety of forms in my study. They demonstrated development in their planning for each IA, as they anticipated a wider range of possible student reasoning and wrote notes on their protocols as reminders to enact responsive teaching moves (e.g., "write exactly what students say" and "ask someone to restate"). In terms of their enactments, TCs demonstrated basic development by giving students time to think about problems or prompts before engaging them in a whole-class discussion. TCs also showed development in responsive teaching in their attempts to enact responsive teaching moves that were new to them (e.g., Ask Different S to Revoice). TCs' lack of awareness of the responsive teaching moves they enacted is an example of a barrier we encountered. Recall that TCs enacted the move $T$ Makes Connection in responsive ways, but their reflections indicated a lack of awareness that the move had been used at all or that the move was relevant in terms of their 
responsive teaching. Either way, this is problematic - if TCs do not recognize when or how the move was used well, it is unlikely that they will be able to enact the move judiciously in the future.

As they rehearsed the IAs, TCs enacted a variety of teaching moves, some more responsively than others. Of particular significance is the finding that moves positioned as responsive in the literature are not always enacted responsively (e.g., Ask $S$ if they Agree/Disagree; Milewski \& Strickland, 2016). This highlights the importance of TCs learning not only that these moves should be enacted, but also that they should be enacted purposefully and judiciously, which contributes to the moves being enacted responsively. The classification scheme used to distinguish between responsive and unresponsive instances of the same move provided a means of understanding the nuances of different types of moves. For example, my teasing apart of revoicing moves into $T$ Revoices, $T$ Clarifies, and $T$ Repeats revealed that while the differences among these moves are subtle, they have a significant impact on the responsiveness of S-T exchanges. T Revoices, a move in which TCs restate a student idea in their own words, is far more likely to be responsive than $T$ Repeats, when TCs repeat a student's words verbatim. Increasing TCs' awareness of the subtle differences among these moves can contribute to their development as responsive teachers. One way to make TCs more aware of those nuances is through instructional coaching.

RQ2 findings. Instructional coaching played an important role in TCs' development as responsive teachers, in part by introducing TCs to teaching moves that they might not have otherwise considered enacting, such as T Prompts S to Take Think Time and Ask Different S to Revoice. By introducing TCs to these moves and then highlighting salient moments in which to enact them, either through directive feedback or compliments for responsive enactments, the 
coach made contributions to TCs' responsive teaching. Coaching also helped TCs refine their use of more common teaching moves, such as T Records and T Further Questions $S$. Although TCs enacted these moves on their own, coaching suggestions about how to enact the moves in responsive ways, along with compliments to TCs for responsive enactments, made further contributions to TCs' development as responsive teachers.

The rehearsal model of teacher education afforded TCs the opportunity to pause their own rehearsals to ask the coach how to proceed in difficult moments. TCs took advantage of these opportunities and demonstrated particular interest in further questioning students responsively. Their questions demonstrated that they had learned what not to do (e.g., ask leading question to elicit a particular word), but were still unsure of how to continue in responsive ways. In this way, it seems that TCs were working to unlearn some of what had been ingrained in them during their years as students, which is also an important step in their development as responsive teachers (Lortie, 1975; as cited in Lampert, 2010, p. 30).

Finally, TCs' perceptions of instructional coaching are an important consideration for TEs engaged in this work. Although three of the four TCs expressed primarily positive perceptions of coaching, Julian's video annotation comments and some interview responses revealed that he viewed some of my feedback as more punitive than instructive, which perhaps prevented him from fully understanding and attending to the reasons I suggested using certain moves at certain times. Without an understanding of why the coach makes particular suggestions, it is less likely that TCs will have the ability to properly enact responsive teaching moves in the school setting.

RQ3 findings. In response to the third research question, TCs generally taught more responsively in schools than during their Teaching Demos, but not as responsively as their IA 
rehearsals in methods. One particular challenge of the School GOP enactment seems to have resulted from the additional planning responsibilities of choosing a problem and establishing goals for the IA. TCs did not perform either of these tasks for any of their IA rehearsals in methods, which added a layer of difficulty beyond the shift from the university to the school setting.

Findings also revealed that TCs who were concurrently enrolled in a school placement seemed better able, or were more willing to, enact responsive teaching moves. In part, this may have been a result of the rapport that the TCs had already developed with their students. Julian, who was not enrolled in a concurrent school placement, enacted the smallest range of responsive teaching moves and generally seemed less at ease in front of the students than the other TCs.

Finally, although TCs enacted many of the same teaching moves in methods and in schools, the ways they enacted those moves sometimes differed. For example, TCs used $T$ Repeats less responsively in school settings. Alternatively, TCs used Ask $S$ if they Agree/Disagree in more responsive, if still somewhat flawed, ways. This may partially be attributed to the differences in the students in methods and in the school setting; perhaps TCs felt that school students generally required more repetition to ensure that ideas were heard. They may also have felt that Ask S if they Agree/Disagree might be useful as means of gathering feedback from the entire class in a short period of time (e.g., Bridget's request that students give a thumbs up/thumbs down to indicate whether they agreed or disagreed with an idea), in addition to the move's potential to further understand student thinking. Further supporting this conclusion, Charity specifically commented on one of her IA rehearsals that she would have questioned students differently if she had been in a school setting, rather than in a room full of math majors. Although TCs' reasons for enacting moves differently in the school setting is not completely 
understood, it is a finding with implications for practice and future research, which will be discussed in the following sections.

\section{Implications for Practice}

The findings from my study have important implications for practice. Based on these findings, I draw several conclusions related to how TEs might better support TCs' planning work and their development in enacting potentially responsive teaching moves in responsive ways. I also make recommendations for how instructional coaches might better attend to responsive teaching in their comments to TCs. Finally, I discuss implications for designing future iterations of the course, specifically including my plans for adjusting the course design in the upcoming semester.

Planning. TCs' planning work affects their responsive teaching. TCs who noted specific responsive teaching moves to be made in particular moments seemed more likely to actually enact those moves. This was true for both their IA rehearsals in methods, as well as their school enactments. Thus, it is worthwhile for TEs to spend some focused time talking with TCs, both as a class and individually, about their planning for IAs and the importance of writing down what they hope to do in their IA enactments.

Specific to their School GOP IAs, TCs had difficulty establishing goals for the IA, likely in part because they were not responsible for establishing the goals for any of their IA rehearsals in methods. Without well-articulated mathematical goals, it is more difficult for TCs to enact the IA in responsive ways. This is not enough of a reason to suggest that TCs write the mathematical goals for all of their IA rehearsals in methods; as TCs are getting acquainted with the rehearsal model of teacher education and learning how to plan for and enact IA, having assigned, well- 
articulated goals enables TCs to focus more squarely on leading mathematical discussions in responsive ways.

However, perhaps as the TCs gain some experience with IA planning and rehearsals, they might benefit from writing goals for the later IAs. Another option would be to ask TCs to rehearse the GOP IA twice - for the second rehearsal, the TE might assign only a broad topic and then require the TC to choose the problem and establish the goals. Either of these scenarios would allow TCs to "rehearse" goal-writing before they establish goals for their school enactments.

Alternatively, the TE might support TCs in writing goals in ways other than requiring them to write goals for their rehearsals in methods. Perhaps the TE would provide the problems and goals for the IAs along with a rationale about their design, which would allow TCs to gain some insight into how the problems and goals were chosen without requiring them to focus their attention on those aspects of planning. The TE might then dedicate some class time to discussing how to write goals as TCs begin planning for their school enactments. This might involve requiring TCs to have their problems and goals approved by both the TE and the TCs' mentor teachers before the enactment takes place. Whatever method is chosen, results from my study indicate that TCs need support in writing goals, particularly as they prepare for school enactments of IAs. Similarly, as discussed in the following section, TCs also require support in enacting teaching moves in responsive ways.

Potentially responsive moves not always enacted responsively. There are also implications related to the finding that teaching moves positioned as responsive in the literature are not always enacted responsively. There seem to be two possible reasons for this: (1) the TC does not have a clear or responsive intent in mind before enacting the move, or (2) the student 
response results in the TC completing the exchange in an unresponsive way. I describe ways the TE might help TCs enact moves more responsively in response to each of these reasons in the following paragraphs.

TEs can assist TCs in being mindful of their intent by highlighting responsive and unresponsive instances of different moves before (using video of other teachers), during (coaching), and after rehearsals (using video of the current TCs). For example, during methods, TCs in the current study tended to ask students if they agreed with correct answers, without the option to disagree. Once students confirmed their agreement, the TCs did not ask why they agreed, but instead simply moved on with the IA. By asking TCs, "What did you hope to learn by asking that question?" TEs can raise TCs' awareness of what might be gained by using moves in particular ways.

In terms of helping TCs enact responsive teaching moves in response to unexpected students responses, TEs may need to introduce these types of responses in the methods course. TCs were more likely to face unexpected student responses during their school enactments than in methods; TCs acting as students during IA rehearsals did their best to comply with the rehearsing TCs' requests. For example, when a TC called on someone in methods to revoice an idea, students put forth their best effort to revoice that idea. This was not always the case with school students during TCs' school enactments—-recall Charity's interactions with David during her School GOP IA. In this instance, Charity felt a tension between validating David's ideas and foregrounding Daphne's correct solution as a means of working toward the goal for her IA. During her rehearsals in methods, these two aims were not in conflict. To better attend to TCs' developing skills in response to these kinds of contributions, TEs could approximate these situations and ensure that each TC had opportunities to negotiate these kinds of conflicting aims 
in the methods classroom. One way TEs might do this is by playing the role of a student to introduce both correct and incorrect unanticipated responses. As additional benefits, TCs could then receive real-time feedback from the coach about other ways to respond in these situations.

Coaching. Throughout the semester, the substance of C-T exchanges was focused on responsive teaching $61 \%$ of the time. It is reasonable that some C-T instances should be unresponsive as TCs may require guidance in more procedural aspects of teaching, such as articulating instructions concisely and understandably. However, it would have been possible for me (the coach) to focus even more coaching interjections on responsive teaching. For example, several of the C-T exchanges related to the IA protocol could have been more focused on responsive teaching. Much of what is called for in each IA is supportive of responsive teaching, but in my interjections, I did not always highlight those purposes for TCs. For example, the IA protocols for GOP and CthenC each mention restating as a possible move TCs might choose to make during their rehearsals. Although I did make some interjections related to Ask Different $S$ to Revoice, TCs did not seem to fully understand the responsive purpose behind that move, as evidenced by their lack of enactments of the move during the Card Sort IA, whose protocol does not explicitly suggest that TCs have someone restate. Being more attentive to foregrounding these responsive aspects of the IAs themselves would help TCs to better understand why the protocol suggests certain moves, which may then help TCs to incorporate those suggestions in school settings.

Findings also revealed that TCs' perceptions of coaching likely impacted the ways and the extent to which TCs took up coaching feedback. To ensure that TCs receive the maximum benefits from coaching, the TE should also take care to explain that instructional coaching is meant to be instructive, not punitive. The purpose of coaching feedback is not to identify what 
TCs do right or wrong, but instead, to raise TCs' awareness of how to enact teaching moves in more responsive ways. Helping TCs understand that purpose may help to ease their apprehension about the process.

Related to TCs' perceptions of coaching, The TE should be mindful of how TCs are taking up feedback and be prepared to make adjustments as necessary. These adjustments might involve holding a one-on-one conference with TCs who seem to feel criticized by coaching feedback. The TE should request regular feedback directly from TCs to gain an understanding of how they are perceiving the feedback. The TE may also want to reflect upon how the feedback is being delivered to determine whether a gentler delivery of feedback would still be effective.

Along these lines, it might be beneficial to require (or more strongly encourage) TCs to comment on why they chose particular moments in response to the video annotation prompts. Without those comments, it was not always clear why TCs made those choices, which hindered my ability to provide them with useful feedback. These comments might also assist the TE in better focusing the whole-class reflection discussions at the end of each IA cycle. Perhaps inviting TCs to comment on each other's videos might promote more of an ongoing conversation around responsive teaching that could be observed by the TE, who could make comments as it seemed appropriate.

Course design. Finally, findings from this study have implications for the general design of future iterations of practice-based methods course, which include the benefits of concurrent enrollment in a school placement and the choice of IAs to be rehearsed.

Concurrent school placement. One finding of this study revealed that Julian was at a disadvantage during his School GOP enactment because he did not have the same opportunities to learn about or develop a rapport with the class of students he taught during School GOP. This 
gives reason to believe that concurrent enrollment in a methods course and a school placement may help support TCs' development as responsive teachers, though there may also be some hindrances (e.g., Charity's resource teacher's use of Ask Different S to Revoice). TCs concurrently enrolled in school placements may be better able to think about how to enact responsive teaching moves in school classrooms because they are working with both groups (methods class and school students) throughout the semester. This also suggests that there would be benefits to asking TCs to enact more than one IA in the school setting-more opportunities to enact and reflect upon their responsive teaching in school settings would arguably improve their responsive teaching abilities for when they are teaching in classrooms of their own.

Choice of IAs. Another implication for course design involves the number and kinds of IAs assigned to TCs. One finding related to TCs' planning work revealed that they had difficulty in establishing goals for their school enactments. One way TCs might be better supported in writing goals would involve asking TCs to rehearse the GOP IA twice—once when the problem and goals are assigned, and then again when they are assigned a broad topic, but are required to choose a problem and write their own goals for the IA. This would allow TCs an opportunity to work through the goal-writing aspect of planning for an IA with some guidance from the TE.

To make time for additional rehearsals of GOP, perhaps not all TCs should enact all four IAs. While this organization proved useful in terms of my research, in practice, the Card Sort and WODB IAs began to feel redundant. This did not necessarily promote responsive teaching because that redundancy may have allowed TCs to be less mindful of the reasons they were further questioning students. As an example, three of the four Card Sort IAs focused on linear functions in some way, which led TCs to ask many of the same follow-up questions (e.g., "And what is the vertical line test again?"). Julian was the final TC to enact a Card Sort focused on 
linear functions. Because two other TCs had previously asked students to explain the vertical line test, when Julian posed the same question, the student responses were a bit less authentic. It also caused me to wonder if Julian asked the question in more of a rote way (because he had seen two other TCs ask that question), and thus, without a clear intent toward responsive teaching, which would be problematic. Although the mathematical content for the WODB IAs varied a bit more, the structure of that IA lends itself to asking the same kinds of questions, which again, may prevent TCs from fully attending to the responsive purposes of the moves they enact. Asking each TC to enact two GOP IAs, one CthenC, and one Card Sort or WODB would allow some more time to be focused on establishing goals while also eliminating some redundancy among the IAs.

Specific to my plans for future iterations of the course, I intend to ask TCs to enact the GOP IA twice in methods. The first time, I plan to assign the prompts and goals, but provide a narrative to explain why the selected problem and goals work well for GOP. The second time, perhaps after midterm, I plan to provide students with a CSO (one that is relevant to their school placement, if possible), but then require that they choose a problem and write the goals. This will allow more scaffolding than was present in the current study, in that TCs will gain experience in doing more extensive planning work while still enacting the IA in the safety of the methods class. This shift will necessitate a change in the other IAs that will be assigned. I anticipate asking each TC to enact CthenC, but asking only half of the class to enact Card Sort, and the other half of the class to enact WODB.

\section{Implications for Future Research}

Both the design and results of my study carry implications for future research. I first discuss some limitations of my study, as some of the research implications are meant to help 
address those limitations in the future. Then, I move on to discuss implications for similar research design and future research in general.

Limitations. The TCs in the study had a fairly narrow range of educational experiences, in that they all attended West Virginia schools as children. There are some benefits to this-West Virginia students traditionally have shown poor performance in mathematics at the secondary level and the current educational climate discourages teachers from moving to West Virginia to teach. Thus, helping secondary mathematics TCs who plan to remain in the state learn to enact responsive teaching is one way to begin to change the landscape of mathematics education and achievement in West Virginia.

However, it seems reasonable to conclude that the similarities in TCs' backgrounds, in terms of the kinds of mathematics teaching they experienced as students, led them to demonstrate some similarities in their development of responsive teaching skills (e.g., none of the TCs thought about giving students time to solve problems on their own during the Teaching Demos). It would be interesting to know how TCs from other places would bring different experiences to bear on their teaching. For example, would they require prompting to give students time to solve the problems on their own? To what extent might TCs from other places have experienced responsive mathematical class discussion as students and how might that impact their own development as responsive teachers? These are questions that cannot be answered by my study.

Another limitation of my work is that the only data I collected from TCs in schools was a single IA enactment. This was necessary because of the circumstances surrounding their school placements and the single-semester methods course. However, more opportunities to enact IAs in 
schools and more video of what they would consider "typical" teaching would provide additional opportunities for reflection.

TCs' reflections on their school enactments were also somewhat limiting, in that only two TCs wrote substantial comments in their video annotations to provide insight into the school setting. These comments have particular significance related to the school enactment because I was not involved in those classrooms. Thus, more opportunities to reflect with TCs on their enactments would be beneficial in moving this research forward. Perhaps this might mean watching the school enactment with the TC, which would allow for pausing the IA to talk about why the TC chose to enact particular moves in particular ways. More insight could also be gained if the TE watched the school enactments before holding a post-interview; then, the TE could identify moments of interest ahead of time and engage the TC in stimulated recall to ask about their choices of teaching moves.

More insight into TCs' decisions for enacting certain moves in certain ways would also be beneficial. For example, in Bridget's school enactment, is it unclear why she chose to enact Ask $S$ if they Agree/Disagree so frequently. It is also unclear why she sometimes chose to ask students why they agreed or disagreed, while at other times she moved on after a class-wide vote. Having a better understanding of TCs' rationale for their enactments of certain moves would allow a deeper understanding of the extent to which they taught responsively; these insights might even provide the field with a more nuanced view of responsive teaching, thereby continuing to move the field forward. This would also contribute to the existing body of research around teacher noticing, which was only at the periphery of my study.

Implications for research design. My study also carries implications for future research design of similar studies. In the sections that follow, I discuss how my labeling schemes, both for 
teaching moves and coaching, contribute to moving this field of research forward. Then, I discuss how combining this kind of research with research on teacher noticing could be powerful for better understanding how TCs develop skill in responsive teaching.

Labeling scheme. The labeling schemes I used for this study contribute to research in mathematics education in several ways. First, the labels I used for S-T instances teased apart similar moves in ways that are more nuanced than previous research (e.g., T Revoices, $T$ Clarifies, T Repeats). Considering teaching moves at this level of detail allows for a deeper understanding of what slight variations in moves can afford TCs, both in general and in their responsive teaching. Future research should continue this work to examine different types of moves in more detailed ways to identify the affordances of variations of those moves.

Second, the labeling scheme I used for C-T instances is new to the field; while previous research discusses affordances and cautions of using coached rehearsals in teacher education, no work had been done to study coaching feedback in more specific ways. More work should be done in this area to guide TEs engaged in this work toward coaching interjections with substances that will help them move TCs toward desired teaching practices, such as responsive teaching. Further research might consider revisions to my labeling scheme, as my study revealed some redundancy. For example, there was significant overlap between the labels $C$ Asks $T$ to Highlight Math Idea and C Asks T to Further Question S. Since it seems reasonable to conclude that the majority of my requests for TCs to further question students were about mathematical ideas, eliminating the label C Asks T to Highlight Math Idea would streamline the labels. However, my labeling scheme provides a starting point from which future researchers might continue this work. 
Finally, although not organized into tabular form, my descriptions of how to determine whether particular teaching moves were used responsively (e.g., T Repeats was considered responsive only if it was used to ensure proper recording of a student's idea) contribute to the field of responsive teaching research. These descriptions helped to demonstrate that teaching moves with the potential to be responsive will not always be enacted in responsive ways, though primarily for a small set of responsive teaching moves (T Clarifies, Ask $S$ if they Agree/Disagree, and Ask S to Add On). Similar work could be done to distinguish between responsive and unresponsive enactments of other teaching moves. Coming to a consensus around these ideas would also contribute to the establishment of a common language with which researchers might discuss responsive teaching moves.

More insight into TCs' thinking. Future iterations of research would benefit from hearing more from TCs about why they enacted certain moves at certain times. While my research shed some light on this aspect of TCs' teaching, I did not deliberately collect data from TCs about their reasons for enacting certain moves. Hearing more from TCs would allow researchers and TEs to better understand what TCs view as salient moments to enact responsive teaching moves, which could then guide further work aimed at helping TCs develop skill in responsive teaching. This would be of particular interest in studying TCs' school enactments, when the researcher may not be fully aware of the contextual issues in the school setting.

Future iterations of this research would also benefit from obtaining a more complete understanding of TCs' perceptions of instructional coaching. There was evidence to suggest that Julian's perceptions of coaching negatively impacted his ability to teach responsively. However, in his direct communication with me, he claimed that he saw the feedback as helpful. This suggests that I might receive more candid feedback from TCs if they were interviewed by a third 
party about their perceptions of my coaching, particularly if I agreed not to listen to those interviews until grades had been submitted at the end of the term. Having a clearer picture of TCs' perceptions of my coaching would be beneficial as I aim to improve my own practice for future iterations of the course.

\section{Conclusions}

My study contributes to a growing body of literature around responsive teaching, as well as literature focused on the use of core practices in practice-based teacher education classes. Research at the intersection of these two ideas is important because responsive teaching has been shown to positively impact student learning, and the use of core practices in teacher education has been shown to positively impact TCs' teaching practice. If we can use core practice to help TCs learn to enact responsive teaching, students will ultimately reap the benefits, which include better understanding of content and more equitable learning experiences for all students. 


\section{References}

Altheide, D. L., \& Johnson, J. M. (1994). Criteria for assessing interpretive validity in qualitative research. In N. K. Denzin \& Y. S. Lincoln (Eds.), Handbook of Qualitative Research (pp. 485-499). Thousand Oaks, CA: SAGE Publications.

Baldinger, E., Selling, S. K., \& Campbell, M. (2015). Linear function sort cards and definitions.

Baldinger, E. E., Selling, S. K., \& Virmani, R. (2016). Supporting novice teachers to lead discussions that reach a mathematical point: Defining and clarifying mathematical ideas. Mathematics Teacher Educator, 5(1). 8-28.

Ball, D. L. (1993). With an eye on the mathematical horizon : Dilemmas of school mathematics, 93(4), 373-397.

Ball, D. L., \& Cohen, D. K. (1999). Developing practice, developing practitioners: Toward a practice-based theory of professional education. Teaching as the Learning Profession: Handbook of Policy and Practice, 3-32. http://doi.org/10.1037/0022-3514.90.4.644

Ball, D. L., \& Forzani, F. M. (2009). The work of teaching and the challenge for teacher education. Journal of Teacher Education, 60(5), 497-511. http://doi.org/10.1177/0022487109348479

Ball, D. L., \& Forzani, F. M. (2011). Building a common core for learning to teach and connecting professional learning to practice. American Educator, 35(2), 17-21. Retrieved from http://web.b.ebscohost.com/ehost/pdfviewer/pdfviewer?sid=ff50f0dc-44a2-4da1884b-5f0d04fe9785@sessionmgr113\&vid=1\&hid=118

Barab, S. \& Squire, K. (2004). Introduction: Design-based research: Putting a stake in the ground.

Boaler, J. \& Brodie, K. (2004). The importance, nature, and impact of teacher questions. 
Proceedings of the twenty-sixth annual meeting of the North American Chapter of the International Group for the Psychology of Mathematics Education, 2, 774-790.

Boerst, T. A., Sleep, L., Ball, D. L., \& Bass, H. (2011). Preparing teachers to lead mathematics discussions. Teachers College Record, 113(12), 2844-2877. Retrieved from http://www.tcrecord.org

Brodie, K. (2011). Working with learners' mathematical thinking: Towards a language of description for changing pedagogy. Teaching and Teacher Education, 27(1), 174-186. http://doi.org/10.1016/j.tate.2010.07.014

Brofenbrenner, U. (1979). The ecology of human development, Cambridge, MA: Harvard University Press.

Campbell, M. (2016). Going Over a Problem Pre-Planner and Protocol for Rehearsal and Enactment.

Campbell, M. (2016). Which One Doesn't Belong Pre-Planner and Protocol. Adapted from Christopher Danielson (http://talkingmathwithkids.com/2015/01/07/building-a-bettershapes-book-2/) and Mary Bourassa (http://wodb.ca)

Campbell, M. P. (2014). Responsive pedagogies of practice: researching an ambitious secondary mathematics teacher education design. (Unpublished doctoral dissertation). Oregon State University.

Chapin, S.H., O’Connor, C., \& Anderson, N. C. (2013). Classroom discussions in math: A teacher's guide for using talk moves to support the Common Core and more, Grades K-6. Sausalito, CA: Math Solutions.

Common Core State Standards for Mathematics. (2017). Standards for mathematical practice. Retrieved from http://www.corestandards.org/Math/Practice/ 
Colestock, A. A., \& Sherin, M. G. (2016). What teachers notice when they notice student thinking: Teacher-identified purposes for attending to students' mathematical thinking. In A. D. Robertson, R. Scherr, \& D. Hammer (Eds.), Responsive Teaching in Science and Mathematics (pp. 126-144). New York, New York: Routledge.

Design-Based Research Collective. (2003). Design-based research: An emerging paradigm for educational inquiry. Educational Researcher, 32(1), 5-8. http://doi.org/10.3102/0013189X032001005

Dotger, B. H. (2015). Core pedagogy: individual uncertainty, shared practice, formative ethos. Journal of Teacher Education, 66(3), 215-226. http://doi.org/10.1177/0022487115570093

Dyer, E. B., \& Sherin, M. G. (2015). Instructional reasoning about interpretations of student thinking that supports responsive teaching in secondary mathematics. ZDM Mathematics Education, 48(1), 1-14. http://doi.org/10.1007/s11858-015-0740-1

Ferguson, B. (2013). Which One Doesn't Belong tiles (equation representations of power and exponential functions). Retrieved from http://wodb.ca/images/graph22.jpg, http://algebrasfriend.blogspot.ca/2014/09/mtboschallenge-week-5-summary.html

Forzani, F. M. (2014). Understanding "core practices" and "practice-based" teacher education: Learning from the past. Journal of Teacher Education, 65(4), 357-368. http://doi.org/10.1177/0022487114533800

Franke, M. L., Webb, N. M., Chan, A. G., Ing, M., Freund, D., \& Battey, D. (2009). Mathematical thinking in elementary school classrooms. Journal of Teacher Education, 60(4), 380-392. http://doi.org/10.1177/0022487109339906 
Ghousseini, H., Beasley, H., \& Lord, S. (2015). Investigating the potential of guided practice with an enactment tool for supporting adaptive performance. Journal of the Learning Sciences, 24(3), 461-497. http://dx.doi.org/10.1080/10508406.2015.1057339

Ghousseini, H., \& Herbst, P. (2014). Pedagogies of practice and opportunities to learn about classroom mathematics discussions. Journal of Mathematics Teacher Education, 79-103. http://doi.org/10.1007/s10857-014-9296-1

Grossman, P. (2011). Framework for teaching practice : A brief history of an idea. Teachers College Record, 113(12), 2836-2843.

Grossman, P., Compton, C., Igra, D., \& Williamson, P. W. (2009). Teaching practice : A crossprofessional perspective. Teachers College Record, 111(9), 2055-2100. http://doi.org/10.1177/0022487109336543

Grossman, P., Hammerness, K., \& McDonald, M. (2009). Redefining teaching, re-imagining teacher education, 602, 273-289. http://doi.org/10.1080/13540600902875340

Grossman, P. L., \& McDonald, M. A. (2008). Back to the future: Directions for research in teaching and teacher education. American Educational Research Journal, 45(1), 184-205. http://doi.org/10.3102/0002831207312906

Grossman, P. L., Valencia, S. W., Evans, K., Thompson, C., Martin, S., \& Place, N. (2000). Transitions into teaching: Learning to teach writing in teacher education and beyond. Journal of Literacy Research, 32(4), 631-662. https://doi.org/10.1080/10862960009548098

Grossman, P.L., Smagorinsky, P., \& Valencia, S. (1999). Appropriating tools for teaching English: A theoretical framework for research on learning to teach. American Journal of Education, 108(1), 1-29. https://doi.org/10.1086/444230 
Haigh, J. (2007). Expansive learning in the university setting: The case for simulated clinical experience. Nurse Education in Practice, 7(2), 95-102. http://doi.org/10.1016/j.nepr.2006.04.013

Hammer, S. D., Goldberg, F., Fargason, S. (2012). Responsive teaching and the beginnings of energy in a third grade classroom. Review of Science, Mathematics, and ICT Education, $6(1), 51-72$.

Jacobs, V. R., Empson, S. B., Krause, G. H., \& Pynes, D. (2015, April). Responsive teaching with fractions. Paper presented at the NCTM Research Conference, Boston, MA.

Jacobs, V. R., Lamb, L. L. C., \& Philipp, R. A. (2010). Professional noticing of children's mathematical thinking. Source: Journal for Research in Mathematics Education Journal for Research in Mathematics Education, 41(2), 169-202. http://doi.org/10.2307/20720130

Joyce, B. R., \& Showers, B. (1981). Transfer of training: The contribution of" coaching". Journal of Education, 163(2), 163-172.

Kazemi, E., Franke, M., \& Lampert, M. (2009). Developing pedagogies in teacher education to support novice teachers' ability to enact ambitious instruction. Crossing Divides: Proceedings of the 32nd Annual Conference of the Mathematics Education Research Group of Australasia, 1, 12-30.

Kazemi, E., Ghousseini, H., Cunard, A., \& Turrou, A. C. (2015). Getting inside rehearsals: Insights from TEs to support work on complex practice. Journal of Teacher Education, 67(1), 18-31. http://doi.org/10.1177/0022487115615191

Kelemanik, G., Lucenta, A., and Creighton, S. J. (2016). Routines for reasoning: Fostering the mathematical practices in all students. Portsmouth, NH: Heinemann. 
Kennedy, M. (1999). The role of preservice teacher education. In L. Darling-Hammond \& G. Sykes, Teaching as the Learning Profession: Handbook of Teaching and Policy (pp. 54-86). San Francisco: Jossey Bass.

Lampert, M. (2010). Learning teaching in, from, and for practice: What do we mean? Journal of Teacher Education, 61(1-2), 21-34. http://doi.org/10.1177/0022487109347321

Lampert, M., Franke, M. L., Kazemi, E., Ghousseini, H., Turrou, A. C., Beasley, H., ... Crowe, K. (2013). Keeping it complex: Using rehearsals to support novice teacher learning of ambitious teaching. Journal of Teacher Education, 64(3), 226-243. http://doi.org/10.1177/0022487112473837

Lampert, M., Ghousseini, H. N., \& Beasley, H. (2015). Positioning novice teachers as agents in learning teaching. Socializing Intelligence through Academic Talk and Dialogue, 349-359.

Lampert, M., \& Graziani, F. (2009). Instructional activities as a tool for teachers' and TEs' Learning. The Elementary School Journal, 109(5), 491-509. http://doi.org/10.1086/596998

Leatham, K. R., Peterson, B. E., Stockero, S. L., \& Van Zoest, L. R. (2015). Conceptualizing mathematically significant pedagogical opportunities to build on student thinking. Journal for Research in Mathematics Education, 46(1), 88-124. http://doi.org/10.5951/jresematheduc.46.1.0088.

Lee, E. (2013). Which One Doesn’t Belong tiles (equation representations of linear functions). Retrieved from http://wodb.ca/images/graph8.JPG

Lincoln, Y. S., \& Guba, E. G. (1985). Naturalistic Inquiry. Newbury Park, CA: SAGE Publications.

Lortie, D. C. (1975). Schoolteacher: A sociological study. Chicago: University of Chicago Press. 
Marks, B. (2013). Which One Doesn't Belong tiles (systems of linear equations). Retrieved from http://wodb.ca/images/graph19.jpg

McDonald, M., Kazemi, E., \& Kavanagh, S. S. (2013). Core practices and pedagogies of teacher education: A call for a common language and collective activity. Journal of Teacher Education, 64(5), 378-386. http://doi.org/10.1177/0022487113493807

Milewski, A. \& Strickland, S. (2016). (Toward) Developing a common language for describing instructional practices of responding: A teacher-generated framework. Mathematics Teacher Educator, 4(2), 126-144.

National Council of Teachers of Mathematics. (2000). Principles and Standards for School Mathematics. Reston, VA: Author.

National Council of Teachers of Mathematics. (2014). Principles to actions: Ensuring mathematical success for all. Reston, VA: Author.

Peercy, M. M., \& Troyan, F. J. (2017). Making transparent the challenges of developing a practice-based pedagogy of teacher education. Teaching and Teacher Education, 61, 26-36. http://doi.org/10.1016/j.tate.2016.10.005

Richards, J., \& Elby, A. (2014). Incorporating disciplinary practices into characterizations of progress in responsive teaching. Journal of Learning Sciences, 1-29.

Richards, J., \& Robertson, A. D. (2016). A review of the research on responsive teaching in science and mathematics. In A. D. Robertson, R. Scherr, \& D. Hammer (Eds.), Responsive Teaching in Science and Mathematics (pp. 36-55). New York, New York: Routledge.

Robertson, A. D., Atkins, L. J., Levin, D. M., \& Richards, J. (2016). What is responsive teaching? In A. D. Robertson, R. Scherr, \& D. Hammer (Eds.), Responsive Teaching in Science and Mathematics (pp. 1-35). New York, New York: Routledge. 
Schon, D. A. (1987). Educating the reflective practitioner. San Franscisco: Jossey Bass.

Sleep, L. (2012). The work of steering instruction toward the mathematical point: A decomposition of teaching practice. American Educational Research Journal, 49(5), 935970. http://doi.org/10.3102/0002831212448095

Sleep, L., \& Boerst, T. A. (2012). Preparing beginning teachers to elicit and interpret students' mathematical thinking. Teaching and Teacher Education, 28(7), 1038-1048. http://doi.org/10.1016/j.tate.2012.04.005

Smith, M. S. \& Stein, M. K. (2011). Five practices for orchestrating productive mathematics discussions. Reston, VA: National Council of Teachers of Mathematics.

Stahnke, R., Schueler, S., \& Roesken-Winter, B. (2016). Teachers' perception, interpretation, and decision-making: A systematic review of empirical mathematics education research. ZDM Mathematics Education, 48(1), 1-27. http://doi.org/10.1007/s11858-016-0775-y

Surti, M. (2013). Which One Doesn't Belong tiles (graphical representations of polynomial functions). Retrieved from http://wodb.ca/images/graph19.jpg

Thompson, J., Hagenah, S., Kang, H. Stroupe, D., Braaten, M. Colley, C., Windschitl, M. (2015). Rigor and responsiveness in classroom activity. Teachers College Record.

Tyminski, A. M., Zambak, V. S., Drake, C., \& Land, T. J. (2014). Using representations, decomposition, and approximations of practices to support prospective elementary mathematics teachers' practice of organizing discussions. Journal of Mathematics Teacher Education, 17(5), 463-487. http://doi.org/10.1007/s10857-013-9261-4 
Van Zoest, L. R., Peterson, B. E., Leatham, K. R., \& Stockero, S. L. (2016). Conceptualizing the teaching practice of building on student mathematical thinking. In M.B. Wood, E. E.

Turner, M. Civil, \& J. A. Eli (Eds.), Proceedings of the 38th annual meeting of the North American Chapter of the International Group for the Psychology of Mathematics

Education, Tucson, AZ.

Vygotsky, L. S. (1978). Communities of practice: Learning, meaning, and identity. Cambridge, England: Cambridge University Press.

Wideen, M., Mayer-Smith, J., \& Moon, B. (1998). A critical analysis of the research on learning to teach: Making the case for an ecological perspective on inquiry. Review of Educational Research, 68(2), 130-178. 


\section{Appendix A: Math 4431 Weekly Schedule}

\begin{tabular}{|c|c|c|}
\hline \multicolumn{3}{|c|}{$\begin{array}{l}\text { MATH } 4431 \text { Course Outline } \\
\text { (Fall 2017) }\end{array}$} \\
\hline & Rehearsals & Other Assignments \\
\hline Week 1 & $\begin{array}{l}\text { Introduce Core Practices } \\
\text { - Eliciting and responding to student } \\
\text { thinking } \\
\text { - Orienting students to one another's } \\
\text { ideas } \\
\end{array}$ & $\begin{array}{l}\text { Check-In Conferences } \\
\text { (by end of Week 2) }\end{array}$ \\
\hline Week 2 & Teaching Demos & Check-In Conferences (by Friday) \\
\hline Week 3 & $\begin{array}{l}\text { Bike \& Truck Task (Video) } \\
\bullet \quad \text { Watch and analyze } \\
\text { Introduce IA: Going Over a Problem }\end{array}$ & Manipulative (Share Session) \\
\hline Week 4 & Rehearsals: Going Over a Problem & \\
\hline Week 5 & Reflect: Going Over a Problem & \\
\hline Week 6 & $\begin{array}{l}\text { Introduce IA: } \\
\quad \text { Contemplate then Calculate }\end{array}$ & $\begin{array}{l}\text { NCTM Illuminations } \\
\quad \text { (Share Session) } \\
\text { Introduce Unit Assignment }\end{array}$ \\
\hline Week 7 & Rehearsals: Contemplate then Calculate & \\
\hline Week 8 & Reflect: Contemplate then Calculate & $\begin{array}{l}\text { Math History Project } \\
\text { Compile information - single timeline }\end{array}$ \\
\hline Week 9 & Introduce IA: Card Sort & $\begin{array}{l}\text { WV TREE Math Resource } \\
\text { (Share Session) } \\
\text { Math History Presentations }\end{array}$ \\
\hline Week 10 & Rehearsals: Card Sort & \\
\hline Week 11 & Reflect: Card Sort & \\
\hline Week 12 & $\begin{array}{l}\text { Introduce IA: } \\
\text { Which One Doesn't Belong }\end{array}$ & \\
\hline Week 13 & $\begin{array}{l}\text { Rehearsals: } \\
\text { Which One Doesn't Belong }\end{array}$ & Unit due (Monday) \\
\hline Week 14 & $\begin{array}{l}\text { Reflect: } \\
\text { Which One Doesn't Belong } \\
\text { GOP Rehearsal in School due (Friday) }\end{array}$ & Article Summaries due (Wednesday) \\
\hline \multicolumn{3}{|c|}{ THANKSGIVING BREAK } \\
\hline Week 15 & Wrap-Up & $\begin{array}{l}\text { Literature Review due (Wednesday) } \\
\text { Check-Out Conferences (Friday) }\end{array}$ \\
\hline
\end{tabular}




\section{Appendix B: Planning Materials for Going Over a Problem}

"Going Over a Problem" Instructional Activity

Pre-Planner

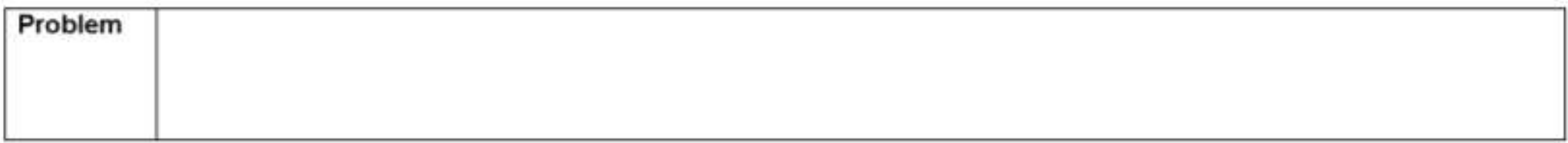

\begin{tabular}{|l|l|}
\hline Goal(s) & What skills, understandings, and/or practices will students gain through their work with this problem? \\
\hline
\end{tabular}

\begin{tabular}{|l|l|}
\hline $\begin{array}{l}\text { Rationale } \\
\text { for how } \\
\text { problem } \\
\text { supports } \\
\text { goal(s) }\end{array}$ & \\
\hline
\end{tabular}

\begin{tabular}{|l|l|}
\hline Ideas to be & \\
highlighted & \\
in work on & \\
problem to & \\
support & \\
goal(s) & \\
\hline
\end{tabular}

\begin{tabular}{|l|l|}
\hline $\begin{array}{l}\text { Ideas that } \\
\text { you will not } \\
\text { pursue in } \\
\text { the moment } \\
\text { if they } \\
\text { emerge }\end{array}$ & $\begin{array}{l}\text { What are the ideas that you will not spend time discussing with students-either because they do not contribute to } \\
\text { your goals or because you are confident all students are proficient with the idea. }\end{array}$ \\
\end{tabular}


284

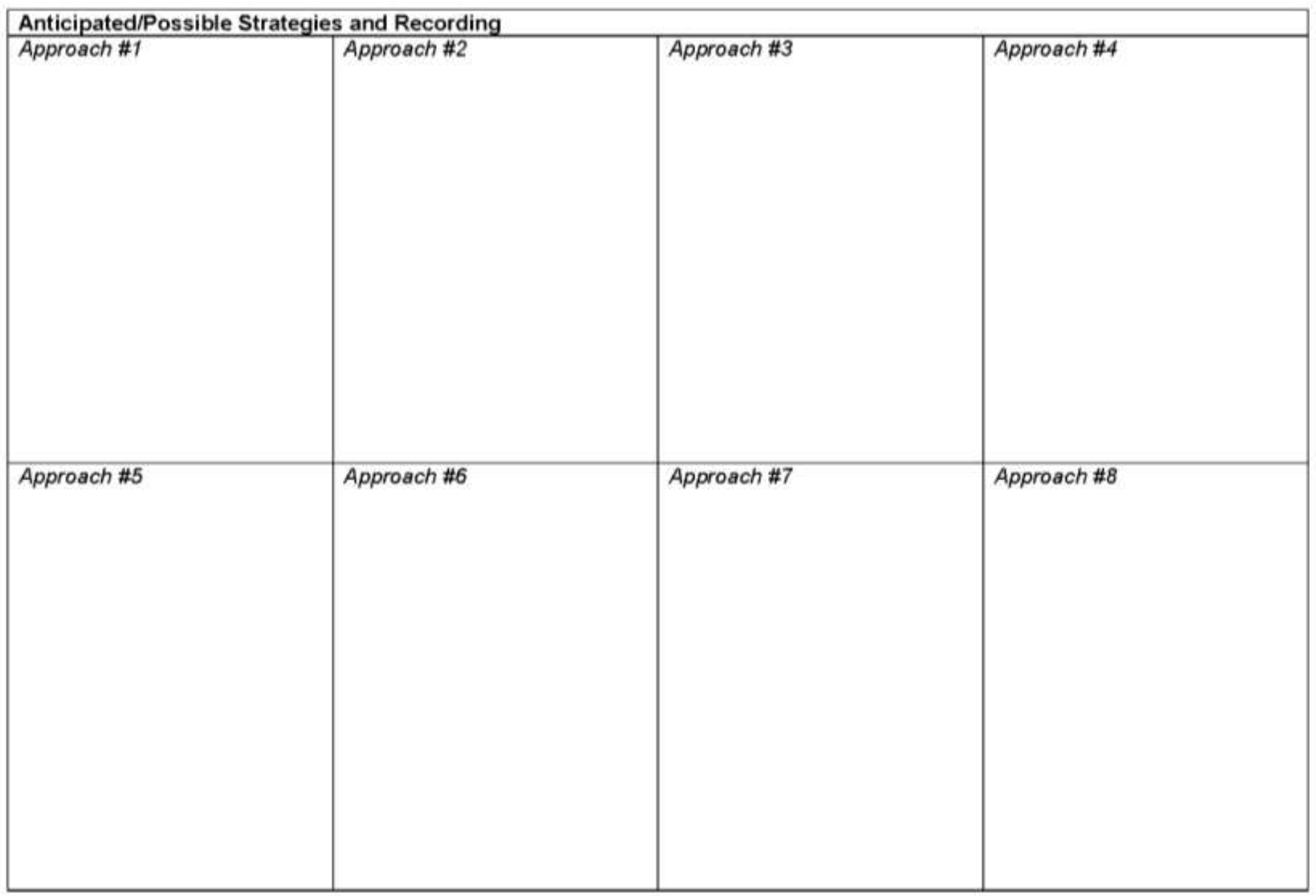

(6) 2016 West Virginia University

Contact: Matthew Campbell (mpcampbell@mati.wvuedu) 


\section{Going Over a Problem Instructional Activity \\ Protocol for Rehearsal and Enactment}

Name:

Date:

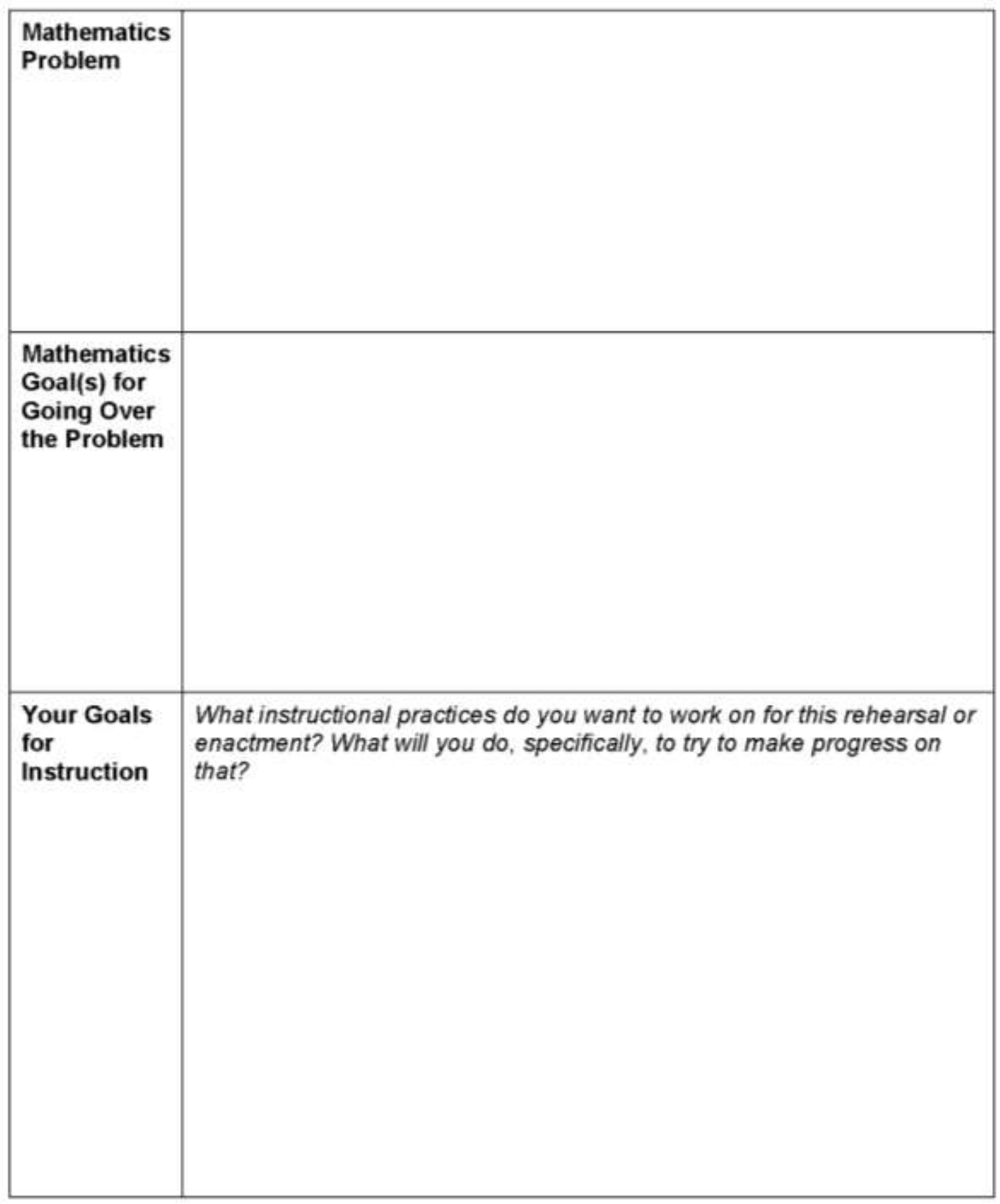




\section{"Going Over a Problem" Instructional Activity Protocol for Rehearsal and Enactment}

\begin{tabular}{|c|c|c|}
\hline Episode/Goal & Protocol of Actions and Moves & Notes \\
\hline $\begin{array}{l}\text { Launch } \\
\text { Problem } \\
\text { (1 minute) }\end{array}$ & $\begin{array}{l}\text { Before posting the problem, orient students to } \\
\text { the mathematics, the context (if there is one } \\
\text { in the problem), and the expectations for } \\
\text { working. This will be contingent on your } \\
\text { context. } \\
\text { [This may involve asking students to provide } \\
\text { ideas on previous days' content or problem } \\
\text { situations, or to restate the expectations of } \\
\text { the forthcoming work.] } \\
\text { Post problem (e.g., document camera, } \\
\text { handout, projector, write on board) and } \\
\text { remind students to begin by working } \\
\text { individually. }\end{array}$ & \\
\hline $\begin{array}{l}\text { Monitor } \\
\text { During } \\
\text { Individual and } \\
\text { Pair Work } \\
\text { (dependent } \\
\text { on the } \\
\text { problem) }\end{array}$ & $\begin{array}{l}\text { Individual monitoring. As students work } \\
\text { individually, circulate around the room, being } \\
\text { sure to not spend too much time with any one } \\
\text { student. You should instead be ensuring that } \\
\text { all students are starting on the task. You } \\
\text { should also get a sense of the strategies } \\
\text { students are using, the stumbling points, and } \\
\text { the solutions they arrive at [all potentially } \\
\text { informed by your anticipating prior to the } \\
\text { enactment]. } \\
\text { Group monitoring. When students have had } \\
\text { enough time to work individually (i.e., all } \\
\text { students have made some good progress, } \\
\text { though before too many students have } \\
\text { finished or become listless), direct students to } \\
\text { share their solutions and strategies with a } \\
\text { partner. Continue your monitoring, listening } \\
\text { and looking for solutions, strategies, and } \\
\text { stumbling points. } \\
\text { Make decisions on the ideas to have shared } \\
\text { in the whole group discussion (and who } \\
\text { should share them), keeping in mind what will } \\
\text { help make progress toward the stated goals. }\end{array}$ & \\
\hline
\end{tabular}




\begin{tabular}{|c|c|c|}
\hline Episode/Goal & Protocol of Actions and Moves & Notes \\
\hline $\begin{array}{l}\text { Debrief: Elicit } \\
\text { Solutions } \\
\text { (<1 minute) }\end{array}$ & $\begin{array}{l}\text { Call for students to come back together. Wait } \\
\text { for full attention. Elicit and record only the } \\
\text { solutions that students got (you should } \\
\text { already know the range of solutions from your } \\
\text { monitoring). Do not give students a sense of } \\
\text { which solution is correct, unless it is the one } \\
\text { solution that all students got. }\end{array}$ & \\
\hline $\begin{array}{l}\text { Debrief: Elicit } \\
\text { and Connect } \\
\text { Reasoning } \\
\text { ( } 2-4 \text { minutes) }\end{array}$ & $\begin{array}{l}\text { You should purposefully elicit and discuss } \\
\text { strategies-to highlight common errors, } \\
\text { highlight key mathematical ideas, and/or } \\
\text { make connections. Be focused on your goal } \\
\text { and also efficient with time. } \\
\text { Call on a student to provide his/her strategy } \\
\text { or reasoning for a given solution. Whatever } \\
\text { you do, this should not be simply be "Who } \\
\text { would like to share how they did it?" } \\
\text { As the student shares, record their reasoning } \\
\text { (you should have thought about recording in } \\
\text { your planning). Elicit additional ideas from the } \\
\text { student as needed. Orient other students to } \\
\text { ideas by asking students to restate, } \\
\text { agree/disagree, ask questions, etc. You } \\
\text { should do this mostly around ideas that are of } \\
\text { core importance to your goals. Revise } \\
\text { thinking/recording as needed. } \\
\text { Elicit additional strategies in the same } \\
\text { manner as needed. }\end{array}$ & \\
\hline $\begin{array}{l}\text { Closure } \\
\text { (1 minute) }\end{array}$ & $\begin{array}{l}\text { Every task, activity, lesson, and unit should } \\
\text { have some kind of closure as a final way to } \\
\text { engage with the key ideas (and to provide } \\
\text { additional information to the teacher). For a } \\
\text { single problem, this would be brief, but should } \\
\text { be an opportunity to reiterate important ideas } \\
\text { from the work that was just completed. } \\
\text { Ask a student to summarize key ideas from } \\
\text { the problem (e.g., how a particular procedure } \\
\text { works, the meaning of a particular term or } \\
\text { idea, whether a particular strategy would } \\
\text { always work, etc.). Follow up with the student } \\
\text { or other students as needed. }\end{array}$ & \\
\hline
\end{tabular}


Appendix C: Planning Materials for Contemplate then Calculate

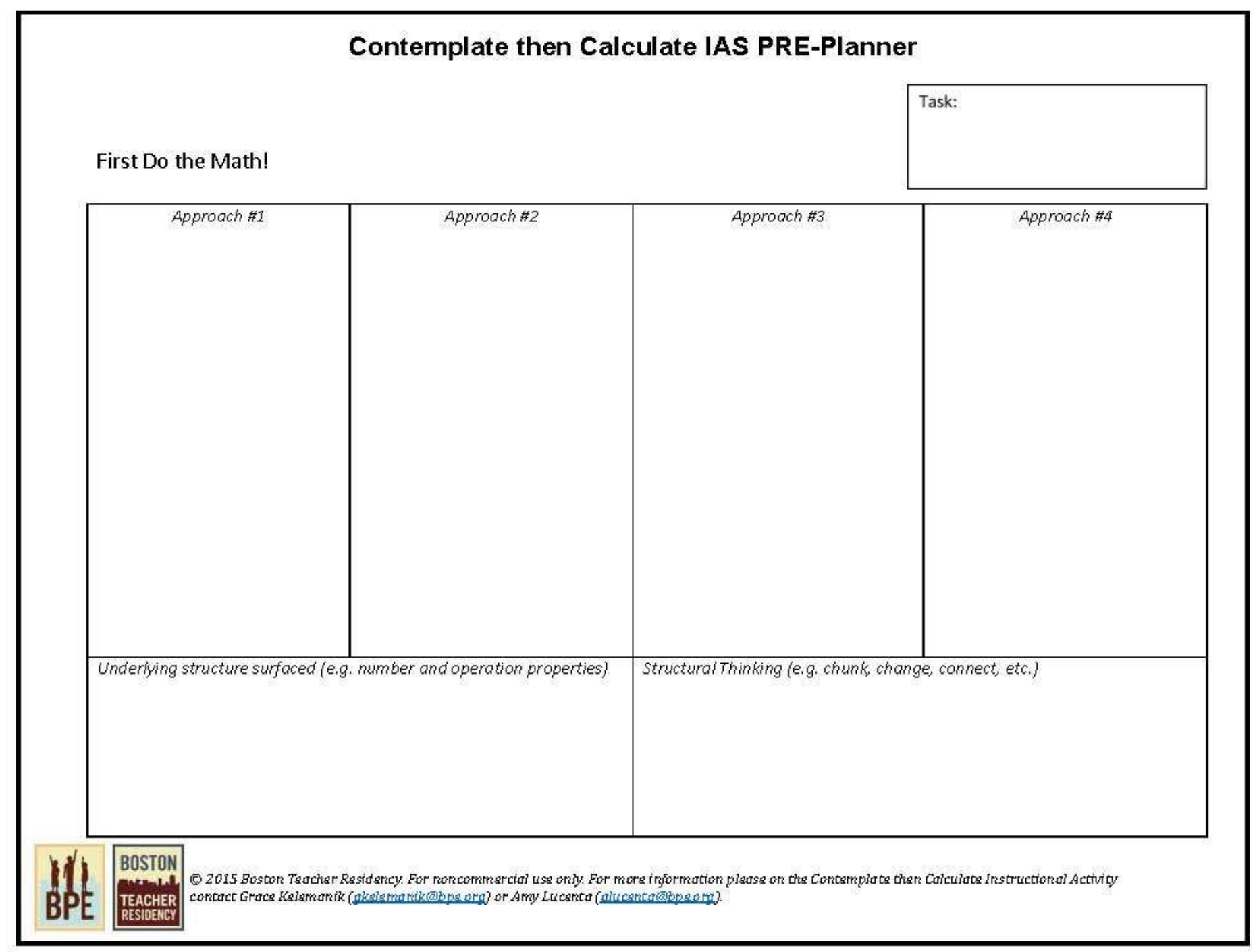




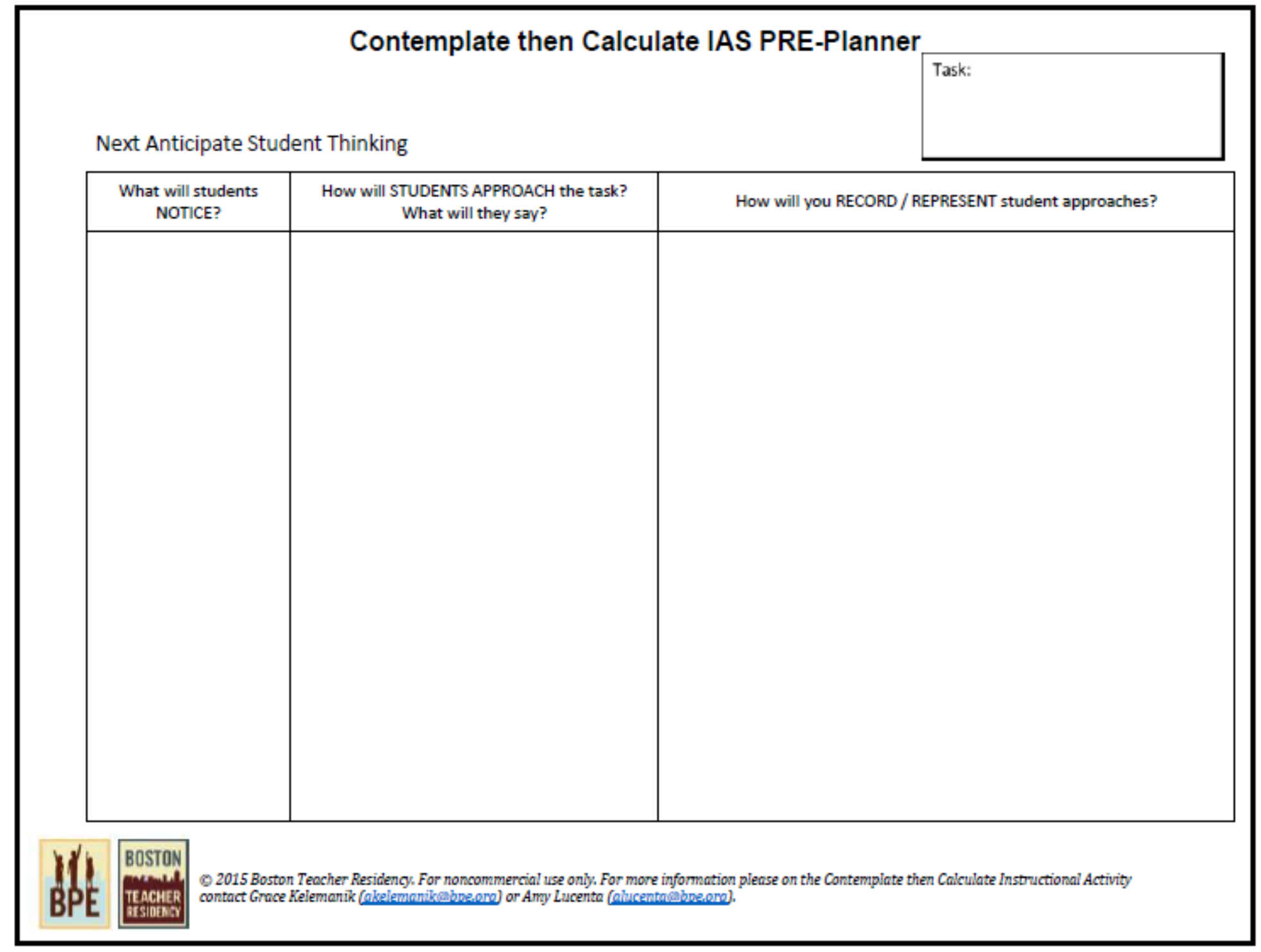




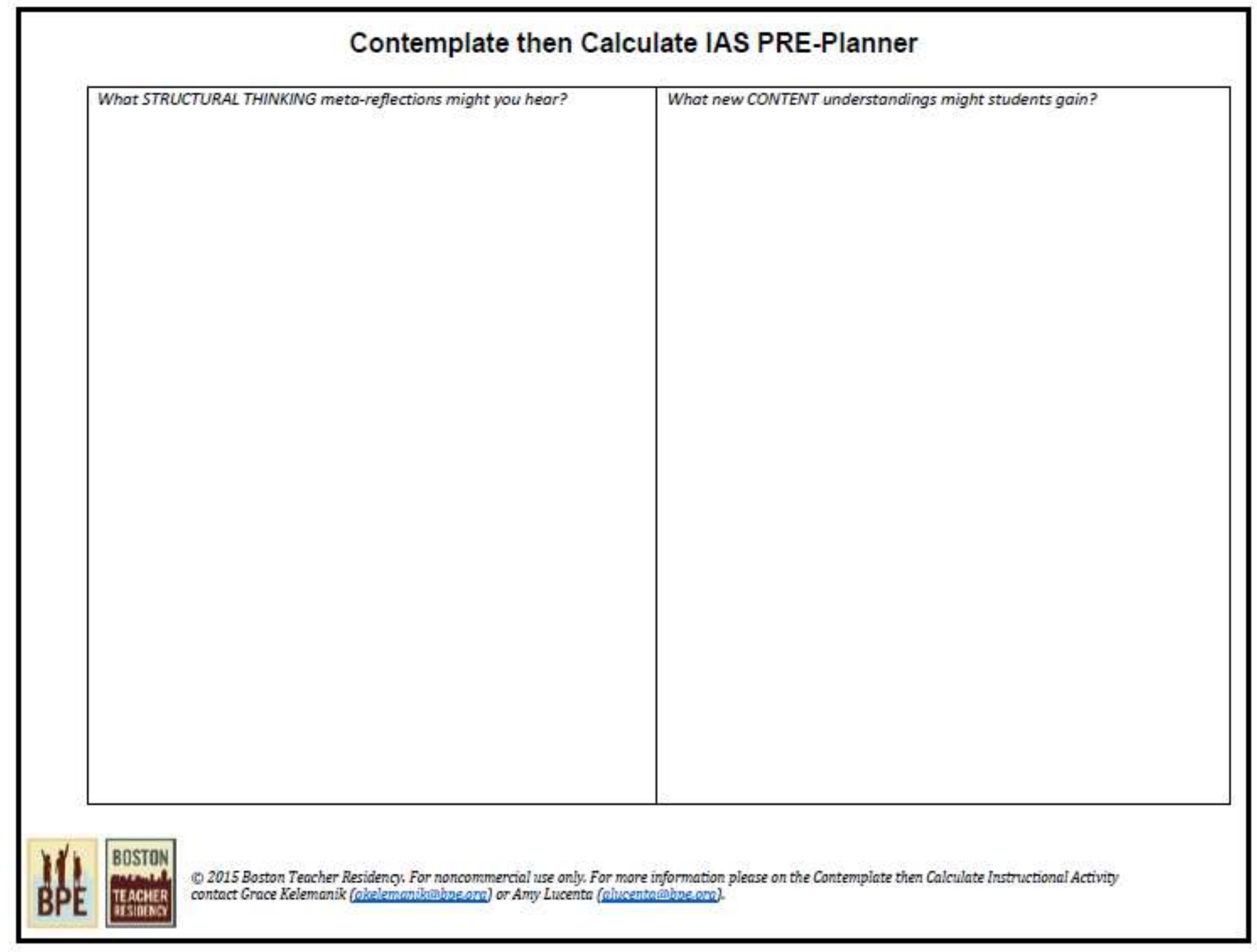




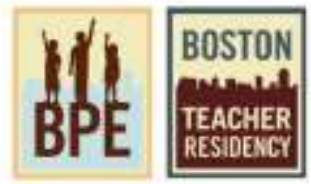

Task:

Planning for a Contemplate Then Calculate Instructional Activity

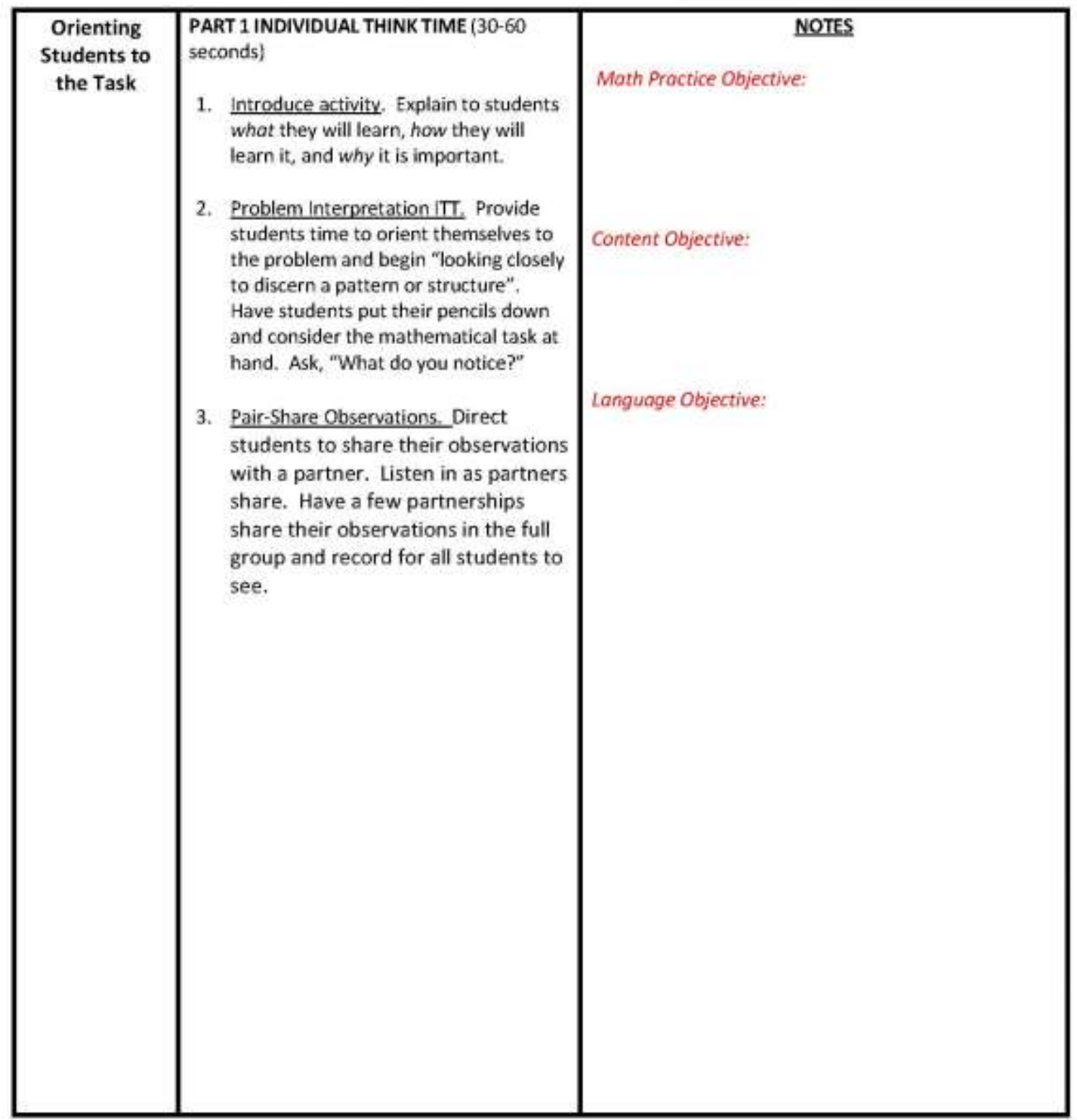

Q 2015 BPE. For noncommercial use only. For more information please on the Contemplate then Calculate Instructional Activity contact

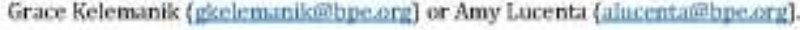



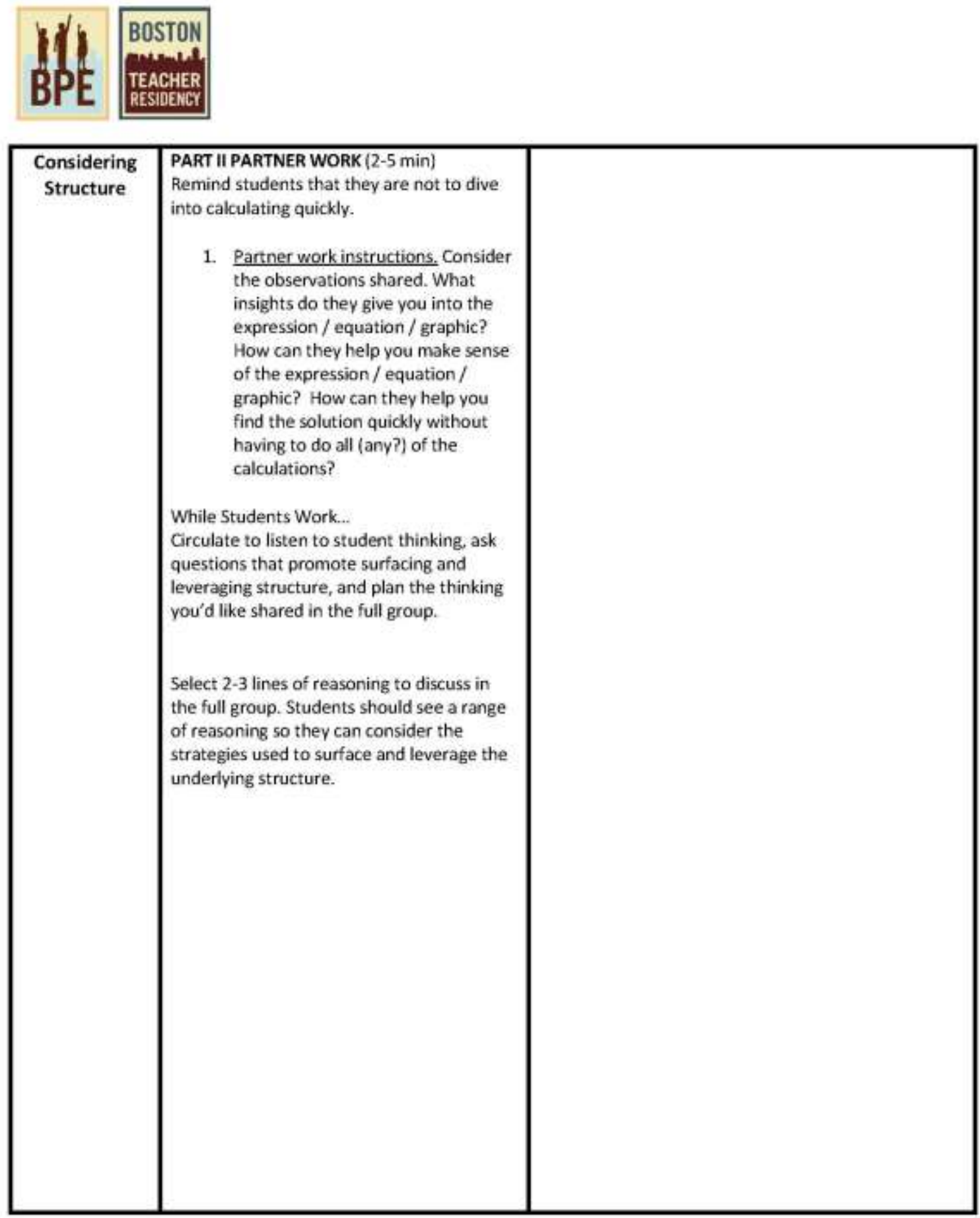

Q4) 2015 BPE. For nencommencial use only. For more information please on the Contemplate then Calculate Instructional Astivity contact

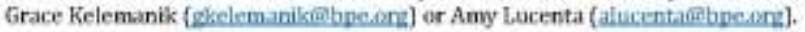




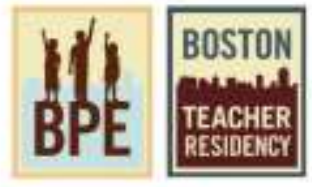

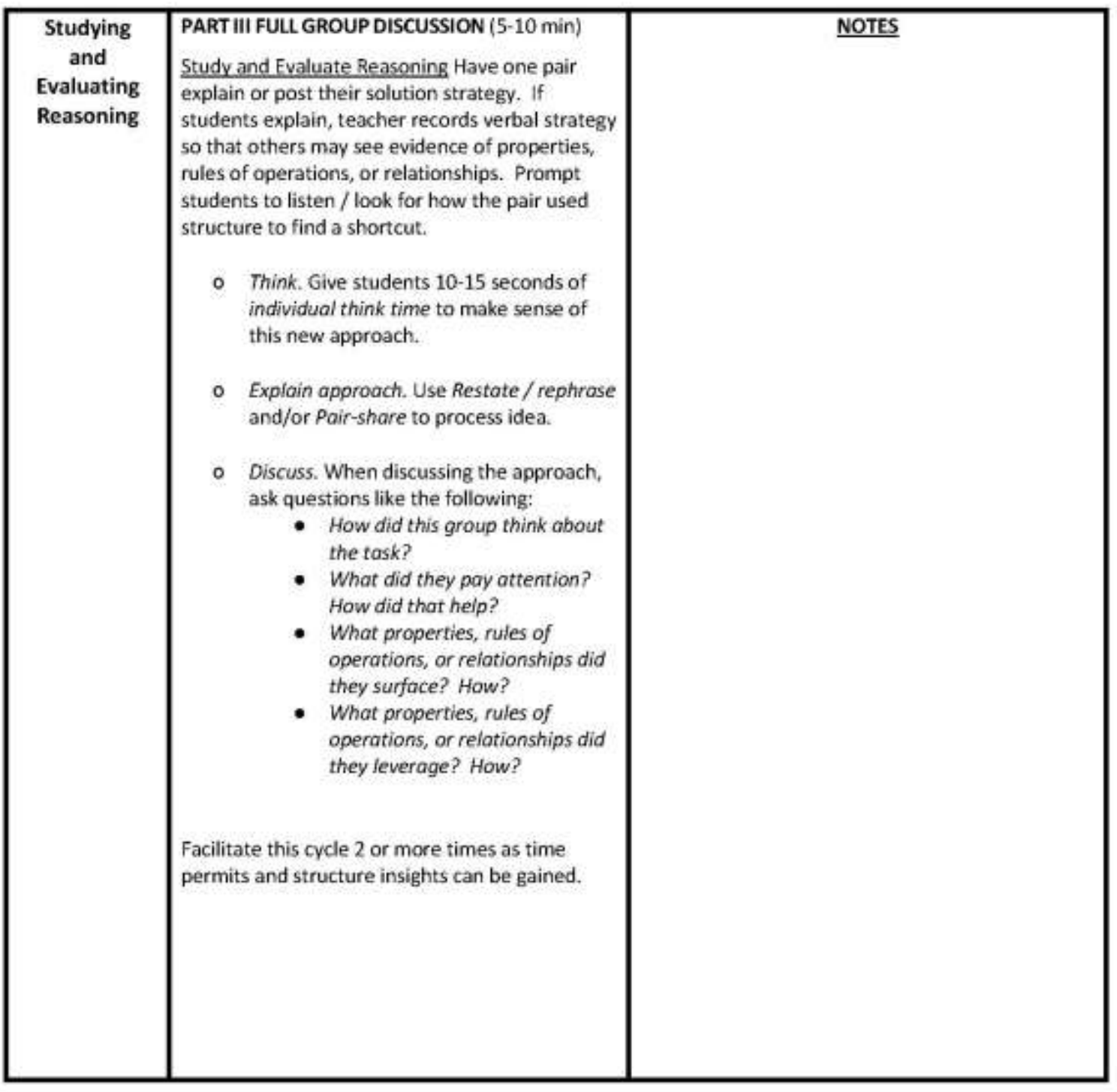

Q) 2015 BPE. For noncommencial use only. For more information please on the Contemplate then Calculate Instructional Activity contact

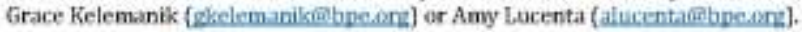




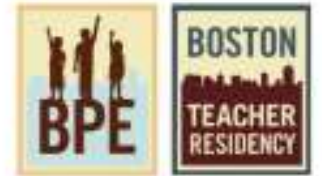

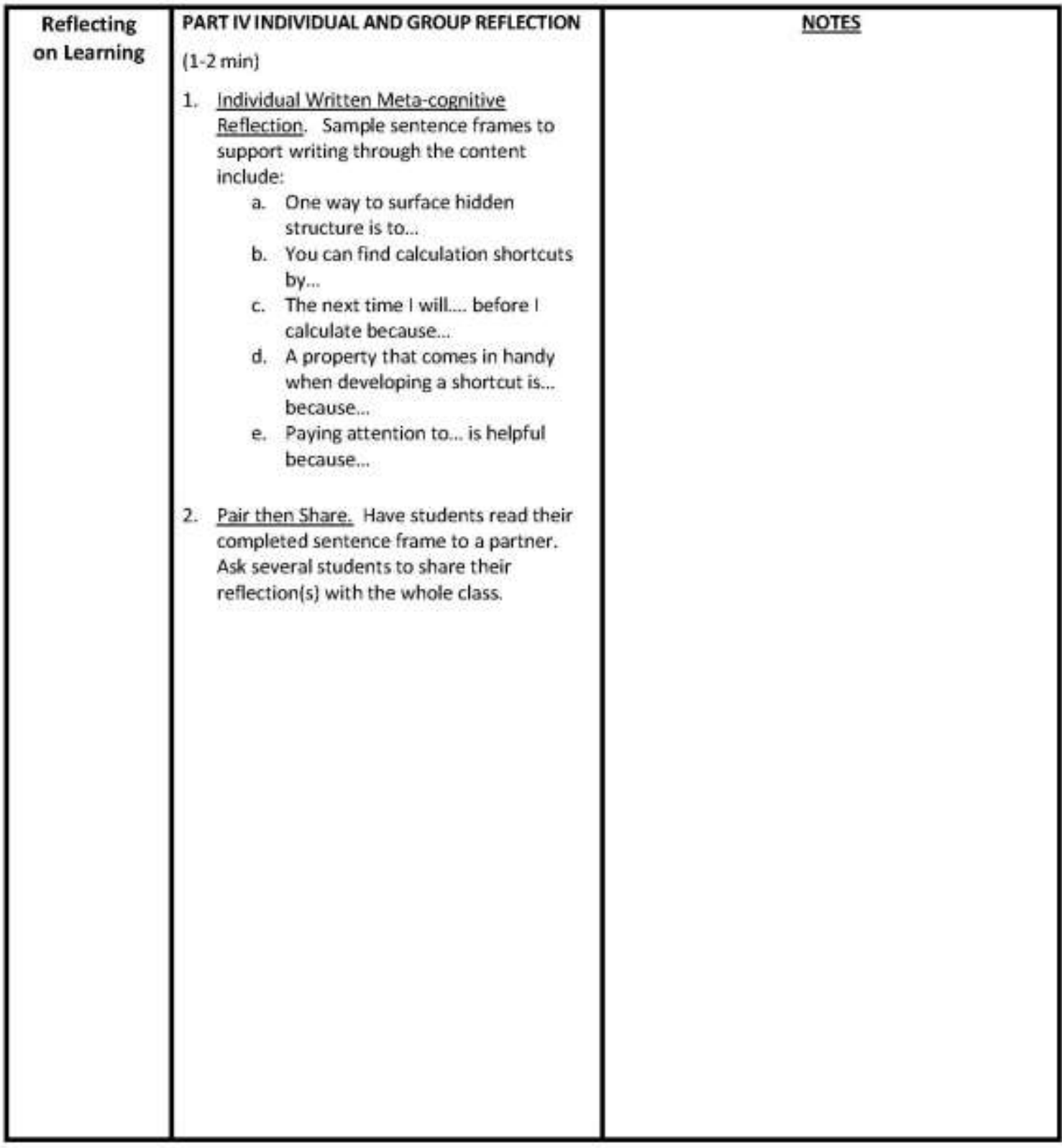

Q4) 2015 BPE. For nencommercial use only. For more information please on the Contemplate then Calculate Instructional Activity contact

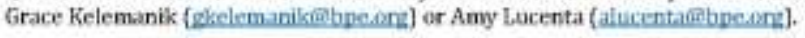




\section{Appendix D: Planning Materials for Card Sort}

\section{Sorting Task Card Analysis}

The following questions are designed to help you get a sense of the potential of the cards in a sorting task to help students work on constructing a definition of a mathematical object or relationship.

You will analyze each card for how it relates to the definition, and you will anticipate how students might respond to each card.

The mathematical goal of the sorting task is to reach the following definition:

\begin{tabular}{|l|l|}
\hline $\begin{array}{l}\text { Card (draw) } \\
\text { Example or non-example? }\end{array}$ & $\begin{array}{l}\text { How does this card relate to the definition? } \\
\text { How might you use it to help move toward the definition } \\
\text { during the discussion? }\end{array}$ \\
\hline & \multicolumn{2}{|l|}{} \\
\hline $\begin{array}{l}\text { Anticipating student thinking: } \\
\text { What will students notice about this card? }\end{array}$ & $\begin{array}{l}\text { Will this card be easy or hard for students to sort? } \\
\text { Is it likely to be sorted incorrectly? }\end{array}$ \\
$\begin{array}{l}\text { What confusions might they have? } \\
\text { Is it a boundary case? Why/why not? }\end{array}$ \\
\hline
\end{tabular}

Note. TCs answered these same questions for each of the cards they were assigned. 
Sorting Task Instructional Activity

(Eliciting and Refining Definitions)

Teacher Talk $=$ Italicized

Teacher Actions = Plain Text

\begin{tabular}{|l|l|}
\hline Mathematical \\
Point of Card \\
Sort (i.e., the \\
definition you \\
are building)
\end{tabular}

\begin{tabular}{|c|c|c|}
\hline $\begin{array}{l}\text { Episode/ } \\
\text { Goal }\end{array}$ & Protocol of Actions and Moves & Notes \\
\hline $\begin{array}{l}\text { Launch } \\
\text { Activity } \\
\text { (1 minute) }\end{array}$ & $\begin{array}{l}\text { Recall that we have talked about } \\
\text { Today our goal is to improve our understanding of } \\
\text { what makes a__ } \\
\text { I'm going to give each group a set of cards. In your } \\
\text { groups, sort the cards into and not } \\
\begin{array}{l}\text { each card as a_. Discuss your reasons for classifying } \\
\text { disagrees about. }\end{array} \\
\text { You may not have time to sort everything. That's okay. } \\
\text { The goal is to think about what makes something a } \\
\begin{array}{l}\text { Teacher hands out sets of the cards to every group } \\
\text { (between 2-4 students per group is fine). }\end{array}\end{array}$ & \\
\hline $\begin{array}{c}\text { Monitor } \\
\text { During } \\
\text { Group } \\
\text { Sorting } \\
\text { (4-5 minutes) }\end{array}$ & $\begin{array}{l}\text { The teacher should pull the group together before any } \\
\text { group has finished and before conversations start } \\
\text { petering out. } \\
\text { The teacher circulates during this time to listen to the } \\
\text { conversations to note common errors, points of } \\
\text { disagreement, and correct conceptions. The teacher } \\
\text { should also note which cards might be useful to } \\
\text { discuss if students don't offer a suggestion. Make a } \\
\text { decision about which student or group might be a } \\
\text { good group to share first. }\end{array}$ & \\
\hline $\begin{array}{l}\text { Debrief: } \\
\text { Eliciting } \\
\text { Examples }\end{array}$ & $\begin{array}{l}\text { Stop your sorting, wherever you are. We're going to } \\
\text { come back together as a class to discuss what makes } \\
\text { something a }\end{array}$ & \\
\hline
\end{tabular}

(C) 2015 - Erin Baldinger, Sarah Kate Selling, \& Matthew Campbell

updated 8/23/2017 


\begin{tabular}{|l|l|l|}
\hline $\begin{array}{c}\text { Reasoning } \\
\text { (2 minutes) }\end{array}$ & $\begin{array}{l}\text { Pause and wait for full attention. } \\
\text { Give me a card that is an example of a } \\
\text { that everybody in your group } \\
\text { agreed on - something that was easy to sort. } \\
\text { Teacher calls on student (this can be strategic based } \\
\text { on the monitoring). Student shares card. } \\
\text { [Record the card in the example column on the board.] }\end{array}$ \\
$\begin{array}{l}\text { If the student does not support his or her choice, the } \\
\text { teacher prompts: } \\
\text { How did your group know that this was an example of } \\
\begin{array}{l}\text { a } \\
\text { [Record student reasoning throughout.] } \\
\text { Continue to elicit and respond to student thinking, and } \\
\text { orient students to one another's thinking. } \\
\text { Ask for a second card that was easy to classify as an } \\
\text { example. Two cards will likely be enough to elicit } \\
\text { several reasons for classifying something as a is disagreement or an error, you do not have to } \\
\text { resolve it here. How might you acknowledge and } \\
\text { record disagreement or errors without resolving them? }\end{array}\end{array}$
\end{tabular} \mid




\begin{tabular}{|c|c|}
\hline $\begin{array}{c}\text { Debrief: } \\
\text { Eliciting } \\
\text { Non- } \\
\text { Examples } \\
\text { and } \\
\text { Reasoning } \\
\text { (2 minutes) }\end{array}$ & $\begin{array}{l}\text { Give me a card that is NOT an example of a } \\
\text { group agreed on - something that everybody in your } \\
\text { Teacher calls on student (this can be strategic based } \\
\text { on the monitoring). Student shares card. } \\
\text { [Record the card in the non-example column on the } \\
\text { board.] } \\
\text { If the student does not support his or her choice, the } \\
\text { teacher prompts: } \\
\text { How did your group know that this was not an } \\
\text { example of } \\
\text { [Record student reasoning throughout.] } \\
\text { Continue to elicit and respond to student thinking, and } \\
\text { orient students to one another's thinking. } \\
\text { Ask for a second card that was easy to classify as a } \\
\text { non-example. Two cards will likely be enough to elicit } \\
\text { several reasons for not classifying something as a } \\
\text { If there is disagreement or an error, you do not have to } \\
\text { resolve it here. How might you acknowledge and } \\
\text { record disagreement or errors without resolving them? }\end{array}$ \\
\hline $\begin{array}{c}\text { Debrief: } \\
\text { Surfacing } \\
\text { Ambiguity } \\
\text { (5-7 minutes) }\end{array}$ & $\begin{array}{l}\text { This time, I'd like to hear a card that your group } \\
\text { disagreed about - something that was difficult to sort. } \\
\text { Teacher calls on student (this can be strategic based } \\
\text { on the monitoring). Student shares card. } \\
\text { [Record the card between the example and non- } \\
\text { example columns on the board.] } \\
\text { What made this difficult to sort? } \\
\text { Elicit arguments for why it might be an example \& } \\
\text { summarize: Who can offer an argument for why this } \\
\text { might be an example of _ } \\
\text { [Record student reasoning throughout.] }\end{array}$ \\
\hline
\end{tabular}




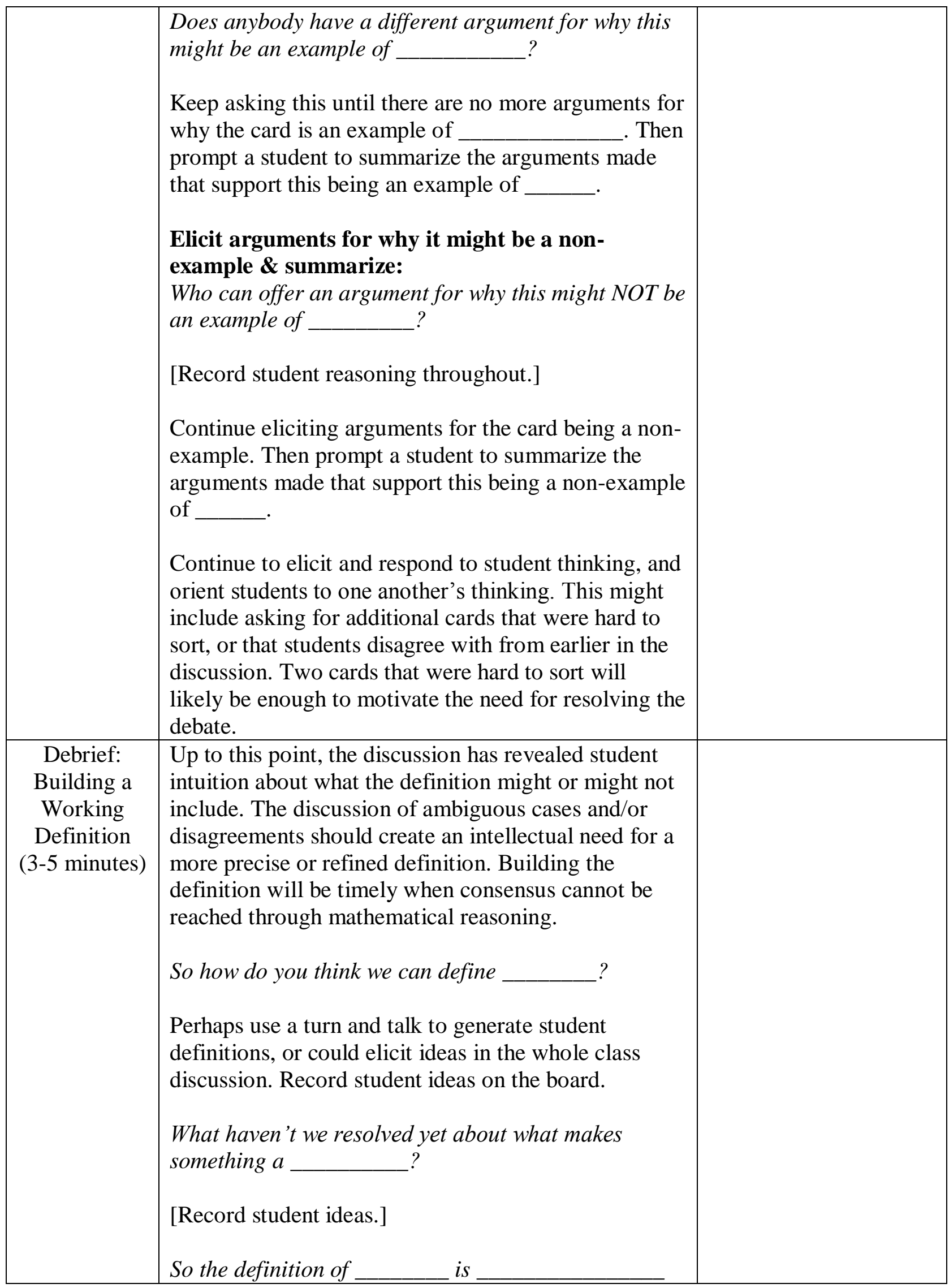

(C) 2015 - Erin Baldinger, Sarah Kate Selling, \& Matthew Campbell

updated 8/23/2017 


\begin{tabular}{|l|l|l|}
\hline & Write or post this on the board. & (2 minutes) \\
\hline $\begin{array}{l}\text { Closure } \\
\text { after this discussion to see where your students are. It } \\
\text { might include determining if something is an example } \\
\text { or non-example, generating examples and non- } \\
\text { examples, or writing the definition in their own words. } \\
\text { You might also consider asking students (individually } \\
\text { or in groups) to sort cards that were not discussed } \\
\text { during the activity. }\end{array}$ & \\
\hline
\end{tabular}

\begin{tabular}{|l|l|l|}
\hline Imagining & $\begin{array}{l}\text { In teaching, not everything goes to plan. Below are } \\
\text { some considerations of dilemmas that might arise } \\
\text { during your card sort discussion. You might consider } \\
\text { how you would respond if this happened, or what you } \\
\text { might do to try to prevent the dilemma from arising: } \\
\text { What if everyone agrees and easily sorts every card? }\end{array}$ & $\begin{array}{l}\text { What if part of the definition is not addressed in the } \\
\text { discussion? } \\
\text { What if student reasoning includes an error related to } \\
\text { the definition? } \\
\text { What if student reasoning includes an error not } \\
\text { related to the definition? }\end{array}$
\end{tabular} \mid




\section{Appendix E: Planning Materials for Which One Doesn't Belong \\ “Which One Doesn't Belong” Instructional Activity \\ Pre-Planner}

Adapted from Christopher Danielson (http://talkingmathwithkids.com/2015/01/07/building-a-better-shapes-book-2/) and Mary Bourassa (http://wodb.ca)

\begin{tabular}{|l|l|}
\hline WODB “Card” & \\
Description & \\
\hline Mathematics & \\
Goal(s) for & \\
Activity & \\
\hline
\end{tabular}

Reasoning about the "Card" - Provide as many reasons as possible for each of the four panels on the card regarding whether the panel is the one that doesn't belong.

\begin{tabular}{|l|l|}
\hline & \\
& \\
& \\
\hline & \\
\hline IA Pre-Planner (1) 2015 West Virginia University & \\
\hline Adapted from Christopher Danielson (http://talkingmathwithkids.com/2015/01/07/building-a-better-shapes-book-2/) & \\
\hline
\end{tabular}




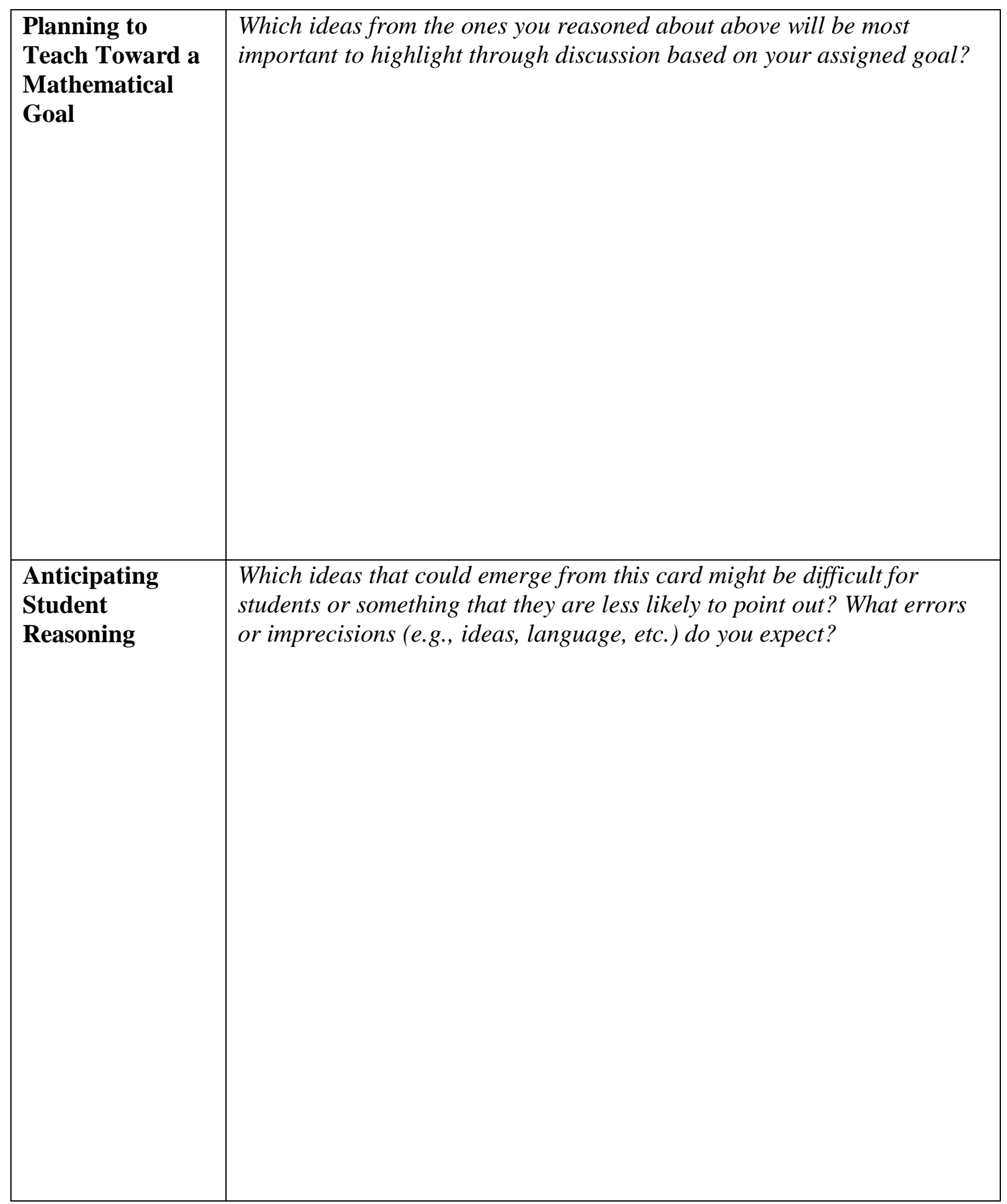

Adapted from Christopher Danielson (http://talkingmathwithkids.com/2015/01/07/building-a-better-shapes-book-2/) and Mary Bourassa (http://wodb.ca)

IA Pre-Planner (C) 2015 West Virginia University 
"Which One Doesn't Belong" Instructional Activity Protocol

Adapted from Christopher Danielson (hetp//takingmutbwithaids cem/2015/01/07/buidine-a-better-shapes-book-2/) and Mary Bourasse (hette//modb ca)

\begin{tabular}{|l|l|}
\hline $\begin{array}{l}\text { WODB "Card" } \\
\text { Description }\end{array}$ & \\
\hline $\begin{array}{l}\text { Mathematics } \\
\text { Goal(s) for } \\
\text { Activity }\end{array}$ & \\
\hline
\end{tabular}

\begin{tabular}{|c|c|c|}
\hline Episode/Goal & Protocol of Actions and Moves & Notes \\
\hline $\begin{array}{l}\text { Launch Activity } \\
\text { (1-2 minutes) }\end{array}$ & $\begin{array}{l}\text { Before posting the "card" } \\
\text { Introduce to students the content they will be } \\
\text { working on and what they are expected to do in } \\
\text { this activity. Emphasize that they are to think } \\
\text { about which of the four graphs/equations/ } \\
\text { numbers/figures doesn't seem to belong with the } \\
\text { others and be able to explain their reasoning. They } \\
\text { may aiso think of ways to consider different } \\
\text { objects as being the one that doesn't belong. } \\
\text { Stress that students are to start by thinking about } \\
\text { this on their own and that they should give a } \\
\text { "silent thumb" when they have had enough time } \\
\text { to make a decision. They will then be prompted to } \\
\text { talk with a partner/small group about their ideas. } \\
\text { Have student(s) restate what they will be } \\
\text { doing/are being asked to do to clarify those } \\
\text { expectations } \\
\text { Post card (e. g., document camera, handout, } \\
\text { projector, write on board) and remind students to } \\
\text { begin by working individually. }\end{array}$ & \\
\hline $\begin{array}{l}\text { Monitor During } \\
\text { Individual and } \\
\text { Pair Work } \\
\text { ( } 3-4 \text { minutes, } \\
\text { though } \\
\text { dependent on } \\
\text { the problem) }\end{array}$ & $\begin{array}{l}\text { Individuol monitoring. Look out at students and } \\
\text { wait for most students to show a "silent thumb". } \\
\text { Group monitoring. When students have had } \\
\text { enough time to think individually, direct students } \\
\text { to share their solutions and strategies with a } \\
\text { partner. Circulate around the room, listening and } \\
\text { looking for solutions, strategies, and stumbling } \\
\text { points. Support groups to not only share ideas, but } \\
\text { also clarify confusion or compare ideas. You might }\end{array}$ & \\
\hline
\end{tabular}

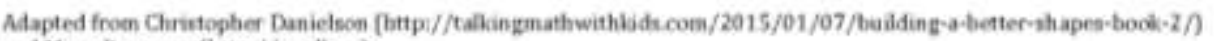
and Mary thourassa (htip///wodbea) 


\begin{tabular}{|c|c|c|}
\hline Episode/Goal & Protocol of Actions and Moves & Notes \\
\hline & $\begin{array}{l}\text { also have groups think of other ways to determine } \\
\text { which one does not beiong. } \\
\text { Make decisions on the ideas to have shared in the } \\
\text { whole group discussion (and who should share } \\
\text { them), keeping in mind what will help make } \\
\text { progress toward the stated goals. }\end{array}$ & \\
\hline $\begin{array}{l}\text { Debrief: Elicit } \\
\text { Ideas About } \\
\text { One of the } \\
\text { Panels } \\
\text { (2-3 minutes) }\end{array}$ & $\begin{array}{l}\text { Call for students to come back together for a } \\
\text { whole class discussion of the problem. Wait for full } \\
\text { attention. } \\
\text { Start by eliciting a line of reasoning from one } \\
\text { student (preferably one you have selected ahead } \\
\text { of time). Ask the student which "panel" they } \\
\text { thought didn't belong and why. } \\
\text { Record relevant ideas on or alongside the panel. } \\
\text { This might include terminology that is used, as well } \\
\text { as notations/markings on the panel. } \\
\text { Press student for more explanation as needed. } \\
\text { Also use taik moves to get other students oriented } \\
\text { to these ideas, especially when the ideas are } \\
\text { important to highlight or dwell on. } \\
\text { One this line of reasoning is complete, elicit other } \\
\text { ideas about how you might consider that panel to } \\
\text { be the one that doesn't belong fas applicable; } \\
\text { again this is best if you know of those ideas ahead } \\
\text { of time). }\end{array}$ & \\
\hline $\begin{array}{l}\text { Debrief: Elicit } \\
\text { Ideas about a } \\
\text { Second Panel } \\
\text { ( } 2-3 \text { minutes) }\end{array}$ & $\begin{array}{l}\text { Elicit ideas about another panel that could be seen } \\
\text { as the one that doesn't belong. Call on a student } \\
\text { to provide that idea and the reasons why. } \\
\text { Note: If no other panels hove been discussed by } \\
\text { students, give them additional time in their groups } \\
\text { to think about a woy to consider one of the other } \\
\text { panels as the "odd one out". } \\
\text { Again, record relevant ideas on or alongside the } \\
\text { panel. Press student for more explanation as } \\
\text { needed. Use talk moves to get other students } \\
\text { oriented to the ideas, especially when the ideas } \\
\text { are important to highlight or dwell on. } \\
\text { Elicit other ideas about this second panel as they } \\
\text { are available. }\end{array}$ & \\
\hline
\end{tabular}

Adagted from Christopher Danielson (http//talkinguathwathidscom/2015/01/07/building-a-better-shapes-bock-2/) and Mary Bourassa (htipi//wodb,ca) 


\begin{tabular}{|c|c|c|}
\hline Episode/Goal & Protocol of Actions and Moves & Notes \\
\hline $\begin{array}{l}\text { Debrief: Elicit } \\
\text { Ideas about } \\
\text { Additional } \\
\text { Panels } \\
\text { (2-3 minutes } \\
\text { each) }\end{array}$ & $\begin{array}{l}\text { Continue to elicit ideas about the third and fourth } \\
\text { panel, as applicable. } \\
\text { Again, record relevant ideas on or alongside the } \\
\text { panel. Press student for more explanation as } \\
\text { needed. Use talk moves to get other students } \\
\text { oriented to the ideas, especially when the ideas } \\
\text { are important to highlight or dwell on. } \\
\text { Elicit other ideas about each panel as they are } \\
\text { available. }\end{array}$ & \\
\hline $\begin{array}{l}\text { Closure } \\
\text { (2-3 minutes) }\end{array}$ & $\begin{array}{l}\text { Close discussion by giving students time to think } \\
\text { individually about a prompt such as: What are } \\
\text { some features of grophs of functions that are } \\
\text { importont to pay attention to and to compare to } \\
\text { other graphs? } \\
\text { Your prompt should highlight your goal, broadly, } \\
\text { and be focused on noticing aspects of a } \\
\text { representation for the sake of knowing more } \\
\text { about that idea or for being able to make } \\
\text { comparisons. } \\
\text { After } 30 \text { seconds or so, have students briefly share } \\
\text { their ideas with a partner/group. Monitor these } \\
\text { ideas and listen for key ideas. } \\
\text { Call students to whole group and quickly elicit } \\
\text { ideas from students. Use talk moves sparingly, } \\
\text { though as needed for highlighting key ideas. You } \\
\text { may also provide some additional narrative in your } \\
\text { revoicing of ideas (e.8., Yes, when we paid } \\
\text { attention to } \\
\text { one of the graphs was different than the others). } \\
\text { Depending on your specific purposes and context, } \\
\text { you might choose other closure routines, such as } \\
\text { an exit ticket or an additional problem or prompt. }\end{array}$ & \\
\hline
\end{tabular}

Mfapted from Christopher Danielson (https/tulkingmattwithbitscoen/2015/01/07/building-a-better-ahapes-bock-2/) and Mary Eourissa (bitpi//wodbca) 


\section{Appendix F: Prompts and Goals for Teaching Demonstrations and Rehearsals}

\section{Teaching Demonstration Prompts}

A student earned grades of 82,70 , and 76 on three tests in a math class. What must the student earn on the fourth test to have an average of 80 for all four tests?

Simone has a cookie recipe that calls for 3 cups of sugar and 4 cups of flour. Simone has a new bag of flour, but only has 1 cup of sugar. How many cups of flour should Simone use with the one cup of sugar to make a smaller batch of cookies?

The perimeter of a rectangle is $54 \mathrm{~cm}$. Its width is $6 \mathrm{~cm}$. What is the length of the rectangle?

The graph of a linear function goes through the points $(-2,-7),(3,-1)$, and $(8,5)$. Write an equation that represents the linear function. 
Going Over a Problem Prompts and Goals (in Methods)

\section{Problem}

Solve for $r$ :

$$
-\frac{2}{3}(r-3)=\frac{4}{5}
$$

\section{Goals}

- Students will recognize the solution(s) to an equation as the value(s) of the variable that makes the statement true.

- Students will solve for an unknown in a given linear equation and will justify their use of method.

- Students will justify why this linear equation, and others, has only one solution.

\section{Problem}

Solve for c:

\section{Goals}

$$
-1+3 c+2==2 c+1+5 c
$$

- Students will recognize the solution(s) to an equation as the value(s) of the variable that makes the statement true.

- Students will solve for an unknown in a given linear equation and will justify their use of method.

- Students will justify why this linear equation, and others, has infinitely many solutions.

\section{Problem}

Solve the following system of equations:

\section{Goals}

$$
\begin{gathered}
2 y-5 x=-28 \\
3 x-2 y=20
\end{gathered}
$$

- Students will articulate the solution(s) to a system of equations is/are the value(s) of all variables that simultaneously make all provided equations true.

- Students will identify the "elimination" method as the most effective way to solve a system of equations when the provided coefficients are equal or opposites.

\section{Problem}

Solve for $\mathrm{x}$ :

$$
13-\frac{4}{3} x>-1
$$

\section{Goals}

- Students will recognize the solution(s) to an inequality as the range of value(s) of the variable that makes the statement true.

- Students will solve a given linear inequality and will justify their use of method.

- Students will contribute to a justification of the procedure to "switch" the inequality when multiplying or dividing by a negative coefficient (e.g., if $\mathrm{x}$ is greater than 2 , then the opposite of $x$ would be less than -2). 
Contemplate then Calculate Prompts

Simplify:

$31 \times 19$

Simplify:

$4 \frac{2}{3} \times 3 \frac{1}{4}$

Order from least to greatest:

$\frac{23}{66}, \frac{4}{11}, \frac{7}{22}$

Simplify:

$0.9 \div 5$ 
Card Sort Prompts (Four Sets of Cards)

\section{Polynomial Function Sort Cards and Definitions}

Definition: A polynomial function is a function that can be written as the sum or difference of monomials, where the coefficients of the variable are real numbers and the exponents of the variable are nonnegative integers.

\begin{tabular}{|c|c|}
\hline$f(x)=2-3 x^{4}$ & $f(x)=\sqrt{x}$ \\
\hline$f(x)=\frac{x^{2}-2}{x^{3}-1}$ & $f(x)=0$ \\
\hline$f(x)=-2 x^{3}(x-1)^{2}$ & $f(x)=\frac{1-x^{2}}{2}$ \\
\hline$f(x)=4 x^{4}+5 x^{2}$ & $f(x)=2 x^{2}$ \\
\hline$f(x)=5 x^{4}-\pi x^{3}$ & $f(x)=(x+4)(x-3)(x+1)$ \\
\hline$f(x)=x(x-6)$ & $f(x)=\frac{1}{3} x^{3}+5 x^{2}-8$ \\
\hline
\end{tabular}




\begin{tabular}{|c|c|}
\hline$f(x)=x^{\frac{3}{2}}-x^{2}+2$ & $f(x)=x$ \\
\hline$f(x)=2(x-7)^{2}$ & $f(x)=5 x^{-2}+4 x^{-1}+9$ \\
\hline
\end{tabular}




\section{Linear Function Sort Cards and Definitions}

Graphical Representation

Definition: A linear function is a function whose graph in the Cartesian plane is a straight linewith a constant slope everywhere - and does not have gaps.

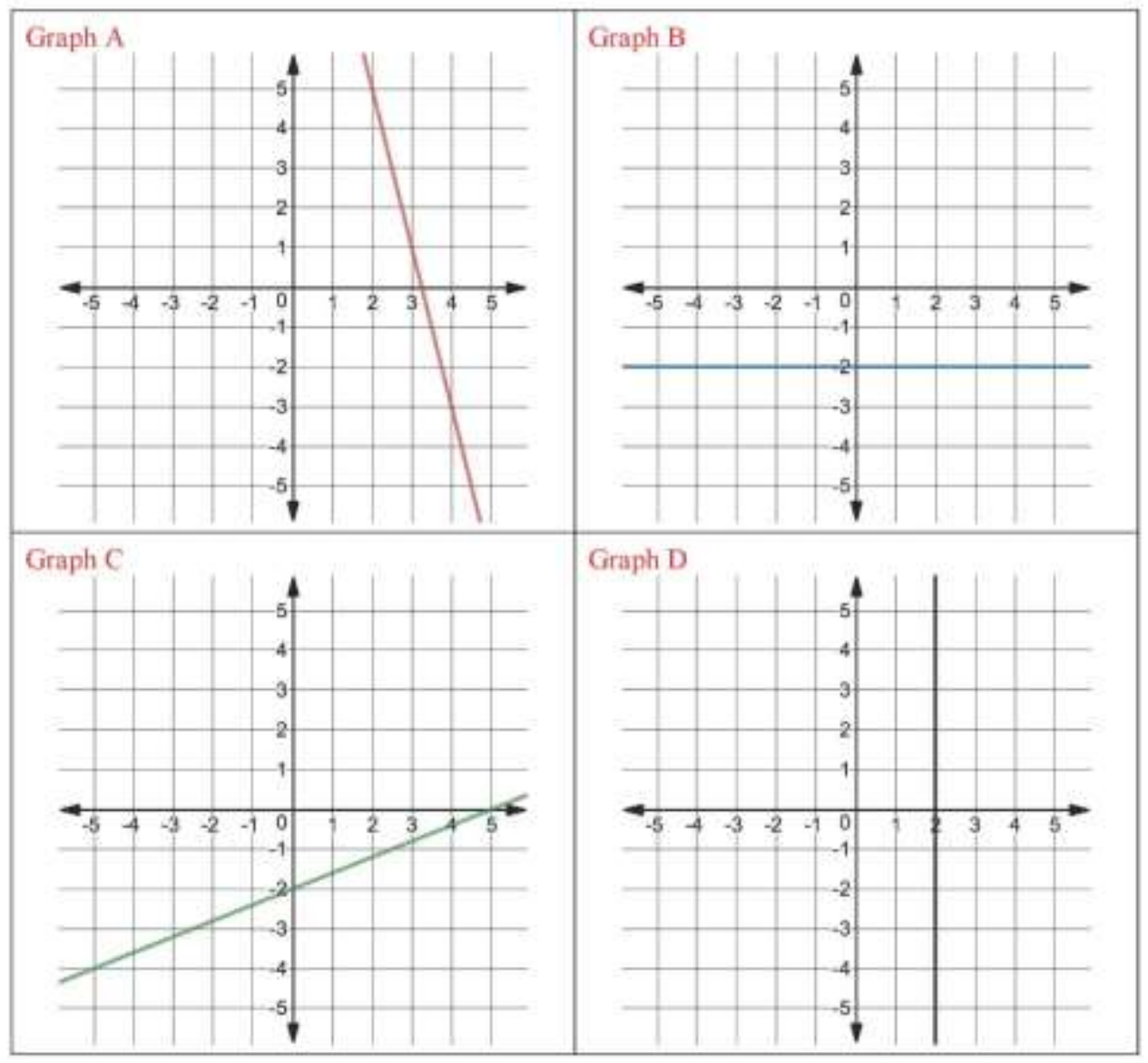




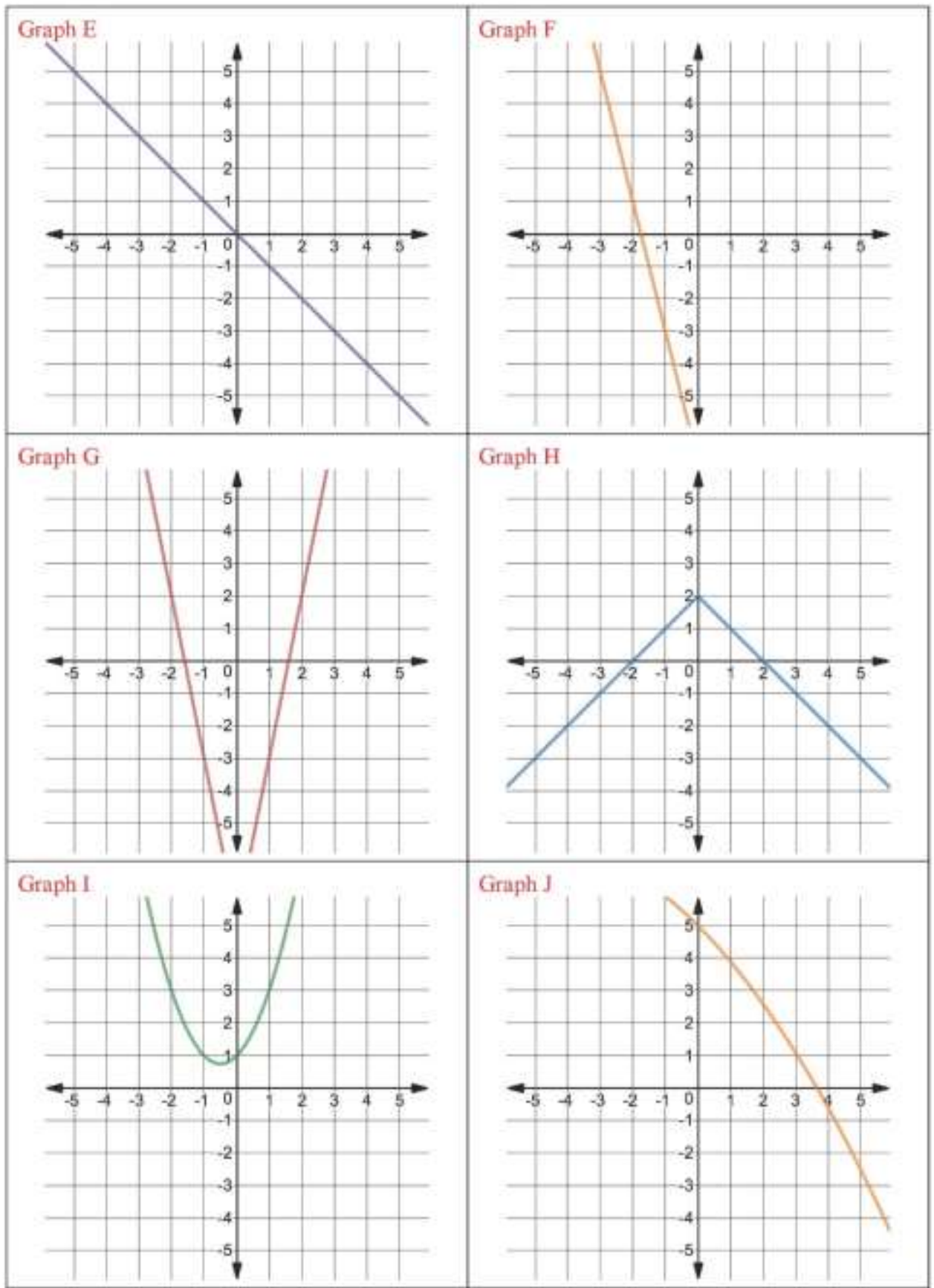

02015 - Erin Haldanger, Sarah Kate Selling, \& Mathew Camphell

updacd 090502017 

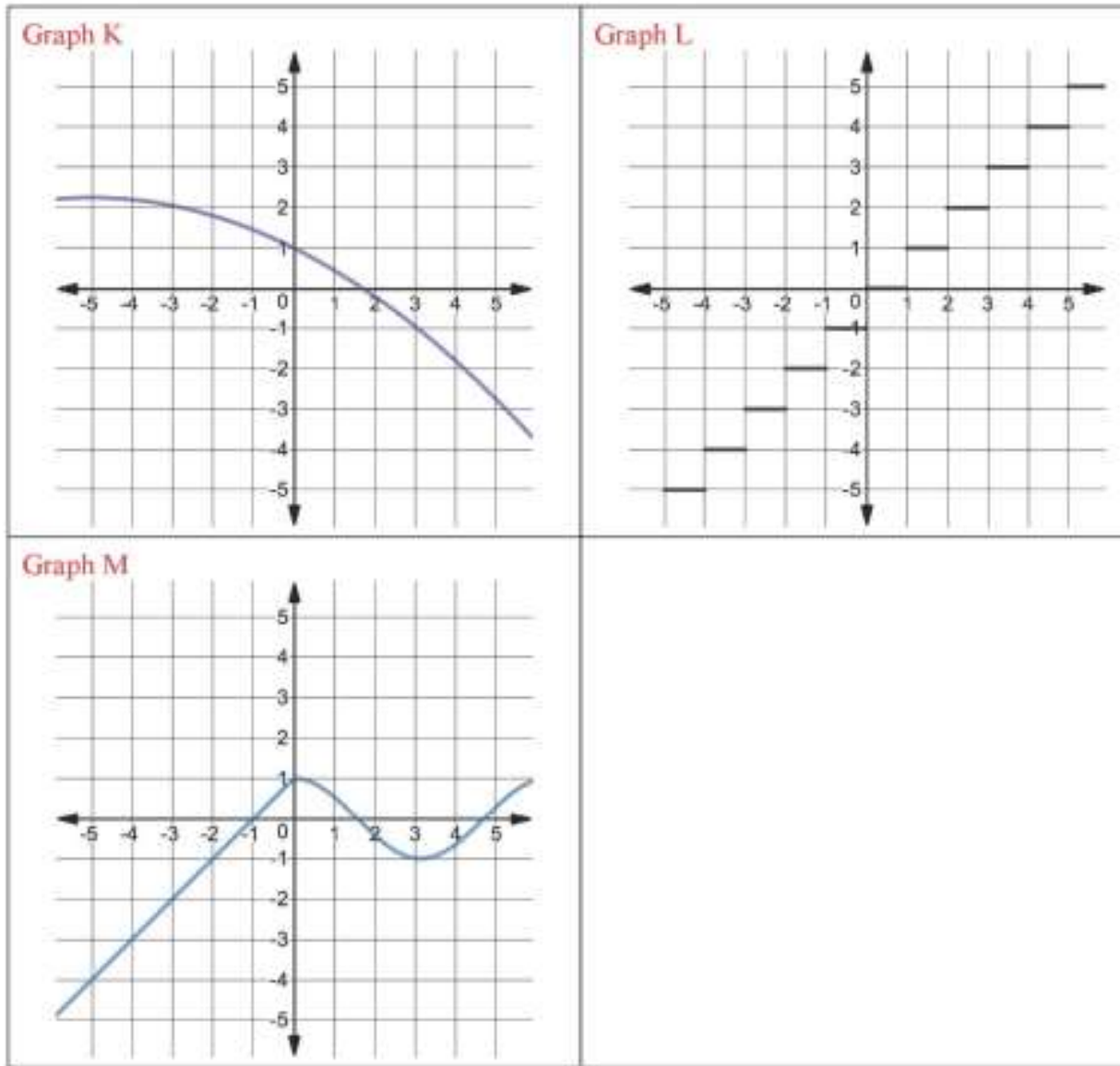
Symbolic Representation

Definition: A linear function is a function that can be represented in the form $f(x)=m x+b$, where $x$ is any real number.

\begin{tabular}{|c|c|}
\hline$y=3 x+5$ & $\frac{y}{x}=2.5$ \\
\hline $11 x+6 y=-23$ & $y=4 x+5 x-6+7$ \\
\hline$y-6=\frac{2}{3}(x+15)$ & $y=8 x^{2}+1$ \\
\hline$x=4 y-7$ & $x=1$ \\
\hline$y=-4$ & $y=\frac{4}{x}$ \\
\hline$y=\frac{2 x-6}{5}$ & $y=x^{2}$ \\
\hline$y=17-5 x$ & $x=2 x+8$ \\
\hline$y=2 x$ & \\
\hline
\end{tabular}




\section{Linear Function Sort Cards and Definitions \\ Tabular Representation}

Definition: A linear function is a function where for any two ordered pairs in the table, the ratio of the change in $y$ to the change in $x$ is constant.

\begin{tabular}{|c|c|c|c|c|c|c|c|c|}
\hline \multicolumn{3}{|c|}{$\mathbf{A}$} & \multicolumn{3}{|c|}{ B } & \multicolumn{3}{|c|}{ C } \\
\hline & $x$ & $\mathrm{y}$ & & $\mathrm{x}$ & $\mathrm{y}$ & & $\mathrm{x}$ & $\mathrm{y}$ \\
\hline & 0 & 2 & & -7 & 13 & & 5 & 18 \\
\hline & 1 & 6 & & -6 & 8 & & 10 & 11 \\
\hline & 2 & 10 & & -5 & 3 & & 15 & 4 \\
\hline & 3 & 14 & & $\begin{array}{l}-4 \\
-3\end{array}$ & $\frac{-2}{-7}$ & & 20 & -3 \\
\hline \multirow[t]{6}{*}{ D } & & & \multicolumn{3}{|l|}{$\mathbf{E}$} & \multicolumn{3}{|l|}{$\mathbf{F}$} \\
\hline & $\mathrm{x}$ & $\mathrm{y}$ & & $\mathrm{x}$ & $y$ & & $\mathrm{x}$ & $y$ \\
\hline & -2 & -7 & & -3 & 5 & & 1 & 10 \\
\hline & -1 & -1 & & 0 & 5 & & 1 & 11 \\
\hline & 1 & 11 & & 3 & 5 & & 1 & 12 \\
\hline & 4 & 29 & & 6 & 5 & & 1 & 13 \\
\hline \multirow[t]{7}{*}{ G } & & & \multirow[t]{7}{*}{ H } & & & \multicolumn{3}{|l|}{ I } \\
\hline & $\mathrm{x}$ & $\mathrm{y}$ & & $\mathrm{x}$ & $\mathrm{y}$ & & $\mathrm{x}$ & $\mathrm{y}$ \\
\hline & 0 & 0 & & -1 & 2.7 & & -2 & 4 \\
\hline & 1 & 1 & & 0 & 0 & & -1 & 2 \\
\hline & 2 & 4 & & 1 & -2.7 & & 0 & 1 \\
\hline & 3 & 9 & & 2 & -5.4 & & 1 & $1 / 2$ \\
\hline & & & & 3 & -8.1 & & 2 & $1 / 4$ \\
\hline
\end{tabular}




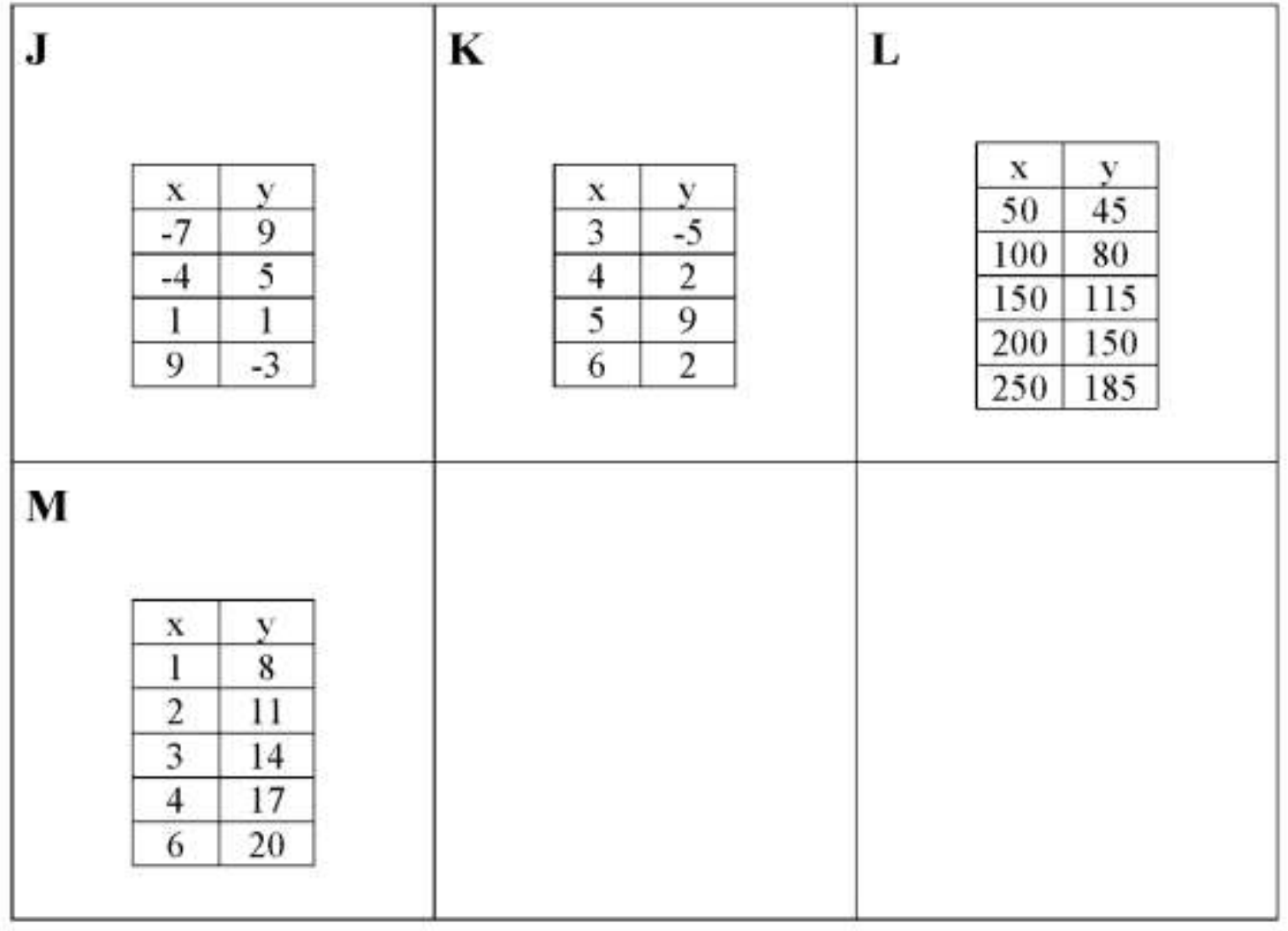


Which One Doesn't Belong Prompts and Goals (Four Sets of Tiles)

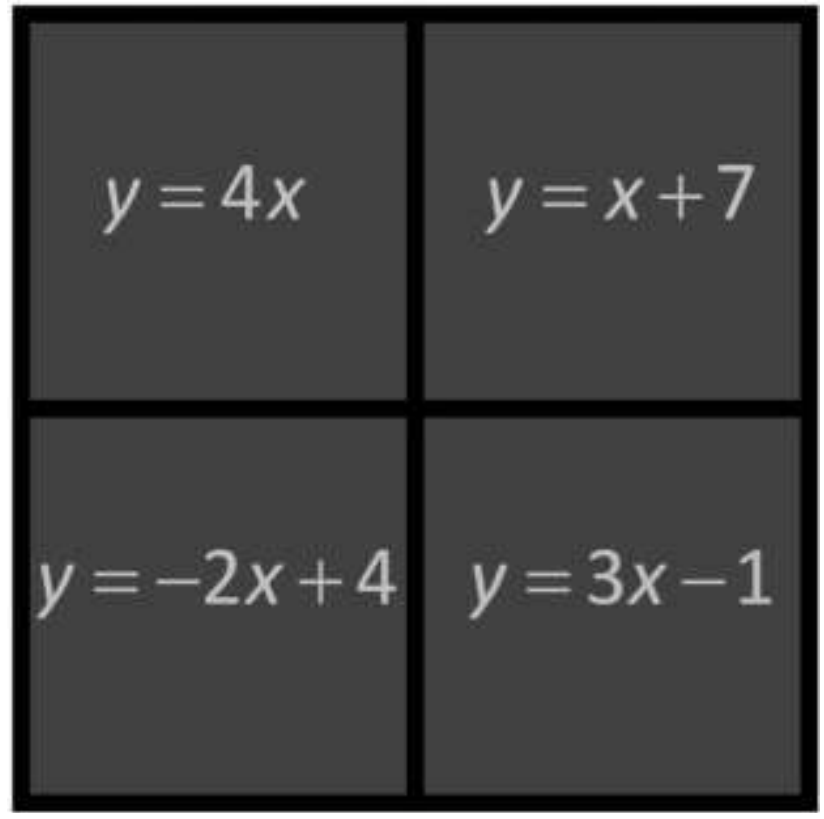

Source: http://wodb.ca/images/graph8.JPG, Author: Erick Lee

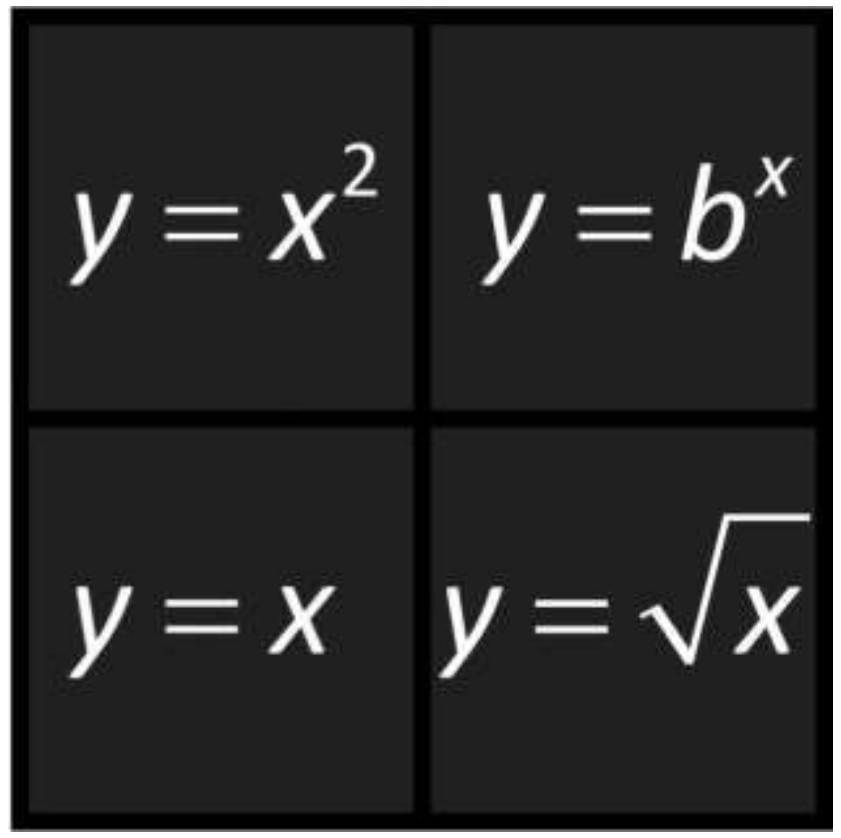

Source: http://wodb.ca/images/graph22.jpg, http://algebrasfriend.blogspot.ca/2014/09/mtboschallengeweek-5-summary.html Author: Beth Ferguson 


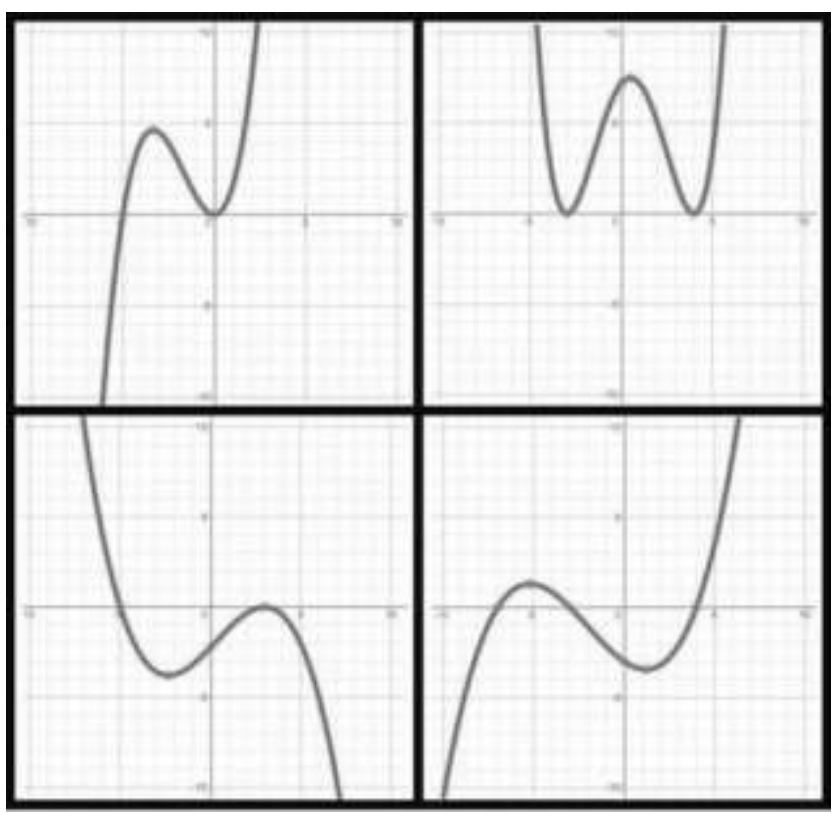

Source: http://wodb.ca/images/graph19.jpg, Author: Mishaal Surti

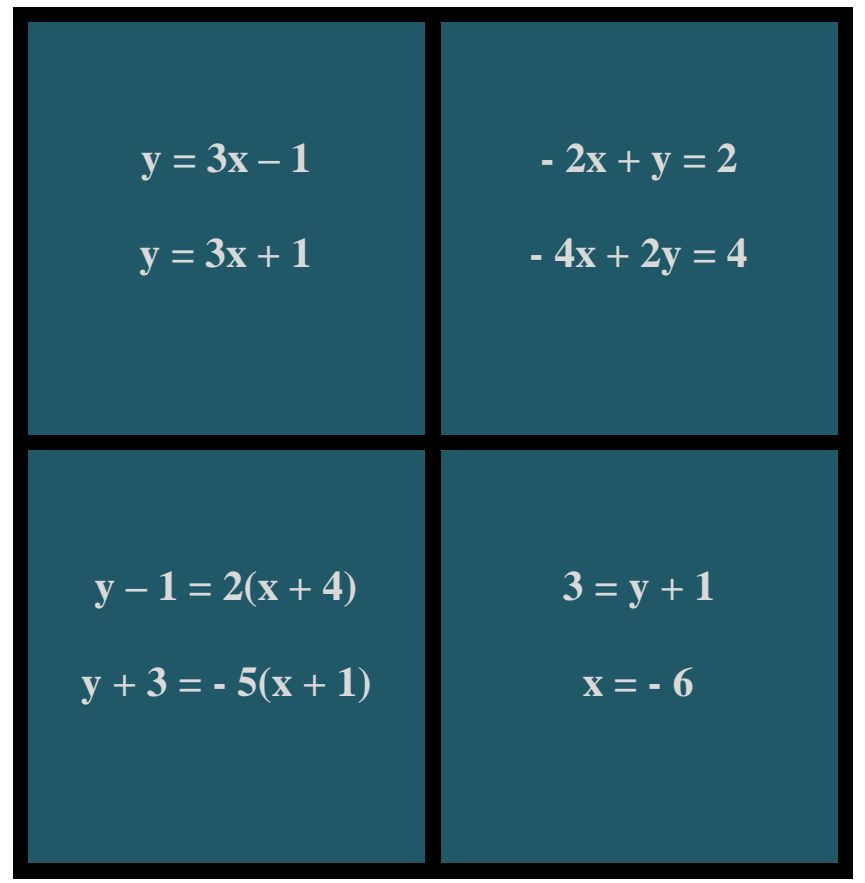

Source: http://wodb.ca/images/graph19.jpg, Author: Brian Marks 
Going Over a Problem Prompts and Goals (School Enactments)

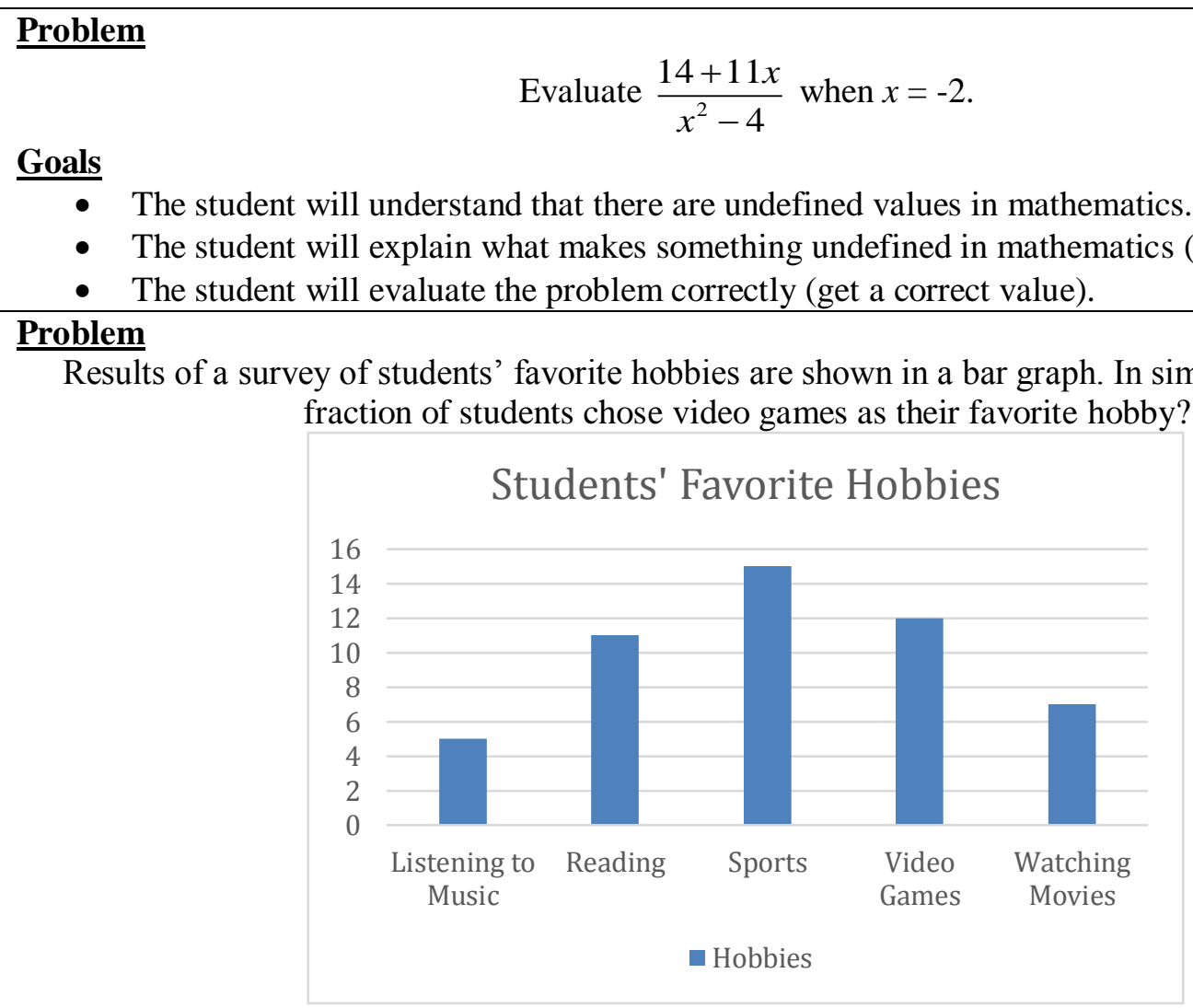

\section{$\underline{\text { Goals }}$}

- Understand how to read a bar graph.

- Understand the denominator represents the whole and the numerator represents a part of the whole.

- Simplify fractions and use reasoning to explain why it is in simplest form.

\section{$\underline{\text { Problem }}$}

\section{Goals}

$$
3(2 x+11)=6 x+33
$$

- Recognize when equations have infinitely many solutions (when both sides are exactly equal).

- Distribute correctly

\section{$\underline{\text { Problem }}$}

Find the mean, median, and mode of the following set:

\section{Goals}

$$
13,21,3,16,8,9,20,30
$$

- Put numbers in order before doing anything (least to greatest)

- What to do with the median when given two numbers

Note. Topics were determined by TCs and their host teachers. At the request of individual TCs, I helped to adjust the problems and goals to fit the IA structure. 


\section{Appendix G: Video Annotation Prompts}

In Vosaic Connect, identify the moments below. Use the "note" feature to explain your choices.

1. Mark moments when you feel you made student reasoning central to the class discussion. Explain why you chose those particular moments to highlight your ability to make student reasoning central to the class discussion.

2. Mark moments when you feel you made progress toward your mathematical goals for the lesson. Explain why you chose those particular moments to highlight your ability to make progress toward a mathematical goal.

3. Mark moments that were difficult (e.g., when you did not know what to do) or a moment in which you made a decision but now feel that another decision would be more appropriate. Explain why you chose those particular moments as challenging or why you felt a different decision would have been more appropriate.

4. Mark moments when you felt supported in your teaching or moments when you felt you needed support in your teaching. Explain why you chose those particular moments as supportive or why you felt you needed support and what supports might have been helpful. 


\section{Appendix H: Interview Prompts}

\section{Prompts for RQ1 (All Interviews)}

1. What do you see as your strengths as a teacher?

2. What teaching skills would you most like to develop this semester?

3. What does the phrase "responsive teaching" mean to you?

\section{Prompts for RQ2 (Mid-and Post-Interviews Only)}

4. Tell me what you think (how you feel) about the rehearsals/coaching? How are they affecting your development as a teacher?

5. Stimulated Recall using clips from rehearsals (as applicable)

a. What were you thinking about when I (the coach) interrupted you?

b. What were the results of my interruption and/or our exchange?

c. Did that change the class discussion? If so, how?

\section{Prompts for RQ3 (Post-Interviews Only)}

6. How did your classroom enactment go?

a. What went as planned? Why did these things go as planned?

b. What didn't go as planned? Why did these things not go as planned?

\section{$\underline{\text { General Prompt }}$}

7. Do you have any questions or comments about the rehearsal process? 